\title{
ULTRA-HIGH-SPEED
}

FLUORESCENCE IMAGING

Erik Gelderblom 


\section{ULTRA-HIGH-SPEED FLUORESCENCE IMAGING}

Erik Gelderblom 


\section{Graduation committee:}

Prof. dr. G. van der Steenhoven (chairman, secretary) University of Twente, TNW

Prof. dr. rer. nat. D. Lohse (supervisor) University of Twente, TNW

Prof. dr. ir. N. de Jong (supervisor) Erasmus Medical Centre

Dr. A.M. Versluis (assistant supervisor) University of Twente, TNW

Prof. dr. J.C.T. Eijkel University of Twente, EWI

Prof. dr. S.G. Lemay University of Twente, TNW

Prof. M.A. Borden University of Colorado

The work described in this dissertation was carried out at the Physics of Fluids Group of the Faculty of Science and Technology of the University of Twente. Financial support for this work was provided by the Dutch Organization for Scientific Research (NWO) and by the Hydro Testing Alliance (HTA).

Dutch title:

Ultra-hogesnelheid fluorescentiemicroscopie

Publisher:

Erik Gelderblom, Physics of Fluids, University of Twente, P.O. Box 217, 7500 AE Enschede, The Netherlands

http://pof.tnw.utwente.nl

e.c.gelderblom@alumnus.utwente.nl

Cover: Liposome-loaded microbubbles, made by the Ghent Research Group on Nanomedicines.

Cover design by: Erik Gelderblom

Printed by: Gildeprint Drukkerijen

(C) Erik Gelderblom, Enschede, The Netherlands 2012.

No part of this work may be reproduced by print, photocopy or any other means without the permission in writing from the publisher. ISBN 978-90-365-3346-1 


\title{
ULTRA-HIGH-SPEED FLUORESCENCE IMAGING
}

\section{PROEFSCHRIFT}

\author{
ter verkrijging van \\ de graad van doctor aan de Universiteit Twente, \\ op gezag van de rector magnificus, \\ prof. dr. H. Brinksma, \\ volgens besluit van het College voor Promoties \\ in het openbaar te verdedigen \\ op vrijdag 20 april 2012 om 16.45 uur
}

door

\section{Erik Carl Gelderblom}

geboren op 23 februari 1984

te Geldermalsen 
Dit proefschrift is goedgekeurd door de promotoren:

Prof. dr. rer. nat. Detlef Lohse

Prof. dr. ir. Nico de Jong

en de assistent promotor:

Dr. Michel Versluis 


\section{Contents}

1 Introduction 1

1.1 Optical imaging . . . . . . . . . . . . . . . . . . . . . 1

1.2 Uttrasound imaging . . . . . . . . . . . . . . . . . . . 2

1.3 Ultrasound contrast agents . . . . . . . . . . . . . . . 3

1.4 Therapeutic applications of contrast agents . . . . . . . . . 4

1.5 Ultra-high-speed fluorescence imaging . . . . . . . . . . 6

1.6 Guide through the chapters . . . . . . . . . . . . . . . . . . 6

2 Brandaris 128 ultra-high-speed imaging facility: 10 years of operation, $\begin{array}{ll}\text { updates and enhanced features } & 9\end{array}$

2.1 Introduction . . . . . . . . . . . . . . . . . . . . . . 10

2.1 .1 System limitations . . . . . . . . . . . . . . . . . . . . . . . 14

2.1 .2 Enhanced teatures . . . . . . . . . . . . . . . . . . . . . . 15

2.2 System description . . . . . . . . . . . . . . . . . . . . . . . . . . . . . . . . 19

2.2 .1 Dedicated timing controller . . . . . . . . . . . . . . . 19

2.2 .2 Region of interest mode . . . . . . . . . . . . . . . . . 19

2.2 .3 Segmented mode . . . . . . . . . . . . . . . . . . 20

2.2 .4 Ultra-high-speed fluorescence imaging setup . . . . . . . 22

2.3 Results . . . . . . . . . . . . . . . . . . . . . . . . . 26

2.3 .1 Microbubble spectroscopy using ROl mode . . . . . . . . 26

2.3 .2 Imaging at multiple time scales using segmented mode . 27

2.3 .3 Ultra high-speed fluorescence imaging of microbubbles . 28

2.4 Discussion . . . . . . . . . . . . . . . . . . . . . . . . . 30

25 Conclusions . . . . . . . . . . . . . . . 32

3 Optical sizing of ultrasound contrast agent microbubbles 33

3.1 Introduction . . . . . . . . . . . . . . . . . . . . . . . 34

3.2 Materials and methods . . . . . . . . . . . . . . . 37

3.2 .1 Microbubbles . . . . . . . . . . . . . . . . . . 37

3.2 .2 Experimental setup $\ldots \ldots \ldots \ldots$

3.2 .3 Measurement protocol . . . . . . . . . . . . . . . . . . 39

3.2 .4 Image analysis . . . . . . . . . . . . . . . . . . . . 40

3.2 .5 Error sources . . . . . . . . . . . . . . . . . . . . 41

B.3 Results . . . . . . . . . . . . . . . . . . . . . . . . . . 47

3.4 Application of the results to an existing data set . . . . . . . . . 48

3.5 Conclusions and discussion . . . . . . . . . . . . . . . 49 
4 US-triggered release from liposome-loaded microbubbles 51

4.1 Introduction . . . . . . . . . . . . . . . . 52

4.2 Materials and methods . . . . . . . . . . . . . . 54

421 Microbubbles . . . . . . . . . . . . . . . . 54

4.2 .2 High-speed fluorescence imaging setup . . . . . . . . 58

4.2 .3 Data analysis . . . . . . . . . . . . . . . . . . . . 60

4.3 Results . . . . . . . . . . . . . . . . . . . . . . . . . . 61

4.3 .1 Rearrangement of fluorescent shell material . . . . . . 61

4.3 .2 Release observations . . . . . . . . . . . . . . . . . 62

4.3 .3 Release distance . . . . . . . . . . . . . . . . . . . . . 69

4.4 Conclusions and discussion . . . . . . . . . . . . . . . 70

5 High-speed fluorescence imaging of bubble-induced sonoporation 73

51 Introduction . . . . . . . . . . . . . . 74

5.2 Materials and methods . . . . . . . . . . . . 76

$5.2 .1 \quad$ Experimental setup $\ldots \ldots \ldots \ldots 76$

5.2 .2 Numerical modell . . . . . . . . . . . . . . . . 79

53 Results . . . . . . . . . . . . . . . . . . . . . . . . . . . 81

5.3 .1 Laser damage threshold . . . . . . . . . . . . . . . . . 81

5.3 .2 Experiment statistics $\ldots \ldots \ldots$. . . . . . . . . . 82

5.3 .3 High-speed fluorescence imaging of PI uptake . . . . . . 83

5.3 .4 Origin of PI uptake . . . . . . . . . . . . . . . . 85

5.3 .5 Timescale of pore formation . . . . . . . . . . . 86

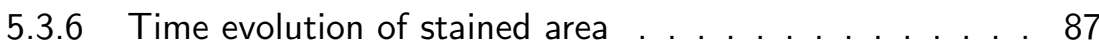

5.3 .7 Pore closure . . . . . . . . . . . . . . . . . . . . 88

5.3 .8 Bubbles interacting with multiple cells . . . . . . . . 90

5.4 Conclusions and discussion . . . . . . . . . . . . . . 90

6 Characterization of polymeric microcapsules for contrast-enhanced photoacoustic imaging 93

6.1 Introduction . . . . . . . . . . . . . . . . . . . . . . . 94

6.2 Experimental parameters . . . . . . . . . . . . . . 95

6.2 .1 Contrast agents . . . . . . . . . . . . . . . . . . . 95

6.2 .2 Experimental setup . . . . . . . . . . . . . . . 96

6.3 Experimental results . . . . . . . . . . . . . . . . . . . . . . . 98

6.3 .1 Pulsed excitation . . . . . . . . . . . . . . . . . . . . 98

63.2 Continuous excitation . . . . . . . . . . . . . . . 101

6.4 Modeling of the vaporization process . . . . . . . . . . . 103

6.4 .1 Activation . . . . . . . . . . . . . . . . . 105

6.4 .2 vapor bubble dynamics $\ldots . . \ldots 106$ 
6.5 Conclusions and discussion . . . . . . . . . . . . . . 109

7 Biodegradable polymeric microcapsules for selective ultrasound$\begin{array}{ll}\text { triggered drug release } & 111\end{array}$

7.1 Introduction . . . . . . . . . . . . . . . . . . . . . 112

7.2 Experimental . . . . . . . . . . . . . . . . . . . . . . . . . . . . . . . . . 114

7.2 .1 Materials . . . . . . . . . . . . . . . . . . . . . . . 114

7.2 .2 Synthesis of PFO-PLLA . . . . . . . . . . . . . . . . 115

7.2 .3 Procedure for preparing PFO-PLLA microcapsules . . . 115

7.2 .4 Ultrasound measurements . . . . . . . . . . . . . 116

7.2.5 Cryo-SEM measurements of PFO-PLLA capsules . . . 116

7.3 Results and discussion . . . . . . . . . . . . . . . . . . . 116

7.4 Conclusions . . . . . . . . . . . . . . . . . . . . . . 122

8 Summary and outlook 123

$\begin{array}{ll}\text { Bibliography } & 127\end{array}$

$\begin{array}{ll}\text { Samenvatting } & 139\end{array}$

\begin{tabular}{ll}
\hline Dankwoord & 143
\end{tabular} 


\section{CONTENTS}




\section{1}

Introduction

\subsection{Optical imaging}

Sight is probably the most powerful method of perception available to humans. When studying an object or an event, the ability to see, allows us to gain a wealth of information instantly. We can observe dimensions, positions, colors etc. very rapidly and we can even store this data. However, the eye is limited in terms of resolution and frame rate and although the human brain can store a vast amount of data, accurate recollection of small image details is impossible. Through the use of optical techniques such as microscopy and telescopy we have overcome the limits of our eyesight by enabling the visualization of objects that are too small or too far away to see with the naked eye. The development of photography and cinematography allows us capture events that occur too quickly, and to store single images and image sequences indefinitely, without any loss of information.

Despite the enormous development of optical imaging techniques over the last few centuries, optical imaging still suffers from several limitations. One major obstacle is scattering of light, which limits the penetration depth of optical imaging in turbid materials. Scattering of light in biological tissue obstructs the use of optical techniques for medical imaging, since the penetration depth is limited to a few millimeters. Other imaging techniques, based on the propagation of X-rays, or ultrasound waves, and magnetic resonance, provide an increased penetration depth of centimeters and higher. As the absorption and scattering of these signals is much lower compared to light rays in biological tissue, the anatomy and physiology of (parts of) the human body can be imaged. 


\subsection{ULTRASOUND IMAGING}

\subsection{Ultrasound imaging}

Ultrasound imaging is the most widely used medical diagnostic imaging technique. Compared to other techniques, such as computed tomography (CT) and magnetic resonance imaging (MRI), ultrasound imaging is relatively inexpensive and the equipment can be used at the bed-side of the patient. Furthermore, ultrasound imaging offers real-time visualization.

The most common application of medical ultrasound is imaging of the fetus in obstetrics. An example is shown in figure [I.]la. As tissue scatters more ultrasound than the amniotic fluid, the outline of the fetus can easily be seen. Bone is an even more efficient scatterer of the ultrasound, allowing for imaging of the spine of the fetus. On the other hand, blood scatters the ultrasound very poorly, which also leads to a contrast in the signal compared to the surrounding tissue, allowing for the visualization of the fetal heart and its chambers (fig. [1] lb). Besides obstetrics, ultrasound diagnostics is used in general for soft tissue imaging and organ perfusion imaging, for instance in echocardiography to visualize the mitral valves and the heart muscle, to assess their morphology and functionality.
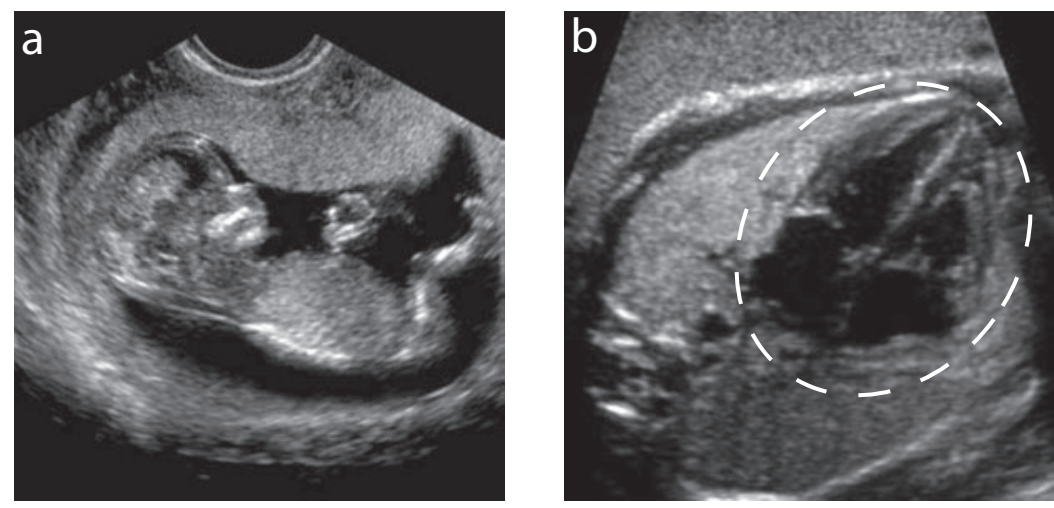

Figure 1.1: Ultrasound image of a fetus (a) and a fetal heart (b), marked by the dashed line.

The applied ultrasound frequencies are in the range of 1 to $50 \mathrm{MHz}$, where the highest frequencies result in the highest spatial resolutions due to a shorter wavelength. At the same time the penetration depth is reduced as the attenuation increases for higher frequencies. Therefore the chosen frequency is a result of the trade-off between imaging depth and resolution. 


\subsection{Ultrasound contrast agents}

Another limitation of ultrasound imaging is the poor contrast echo obtained from blood. In echocardiography for instance, an enhanced blood-to-tissue ratio would make localization of a myocardial infarction easier. The visibility of the blood pool can be enhanced by introducing a contrast agent. Moreover, the obtainable penetration depth is increased. The agent consists of gas-filled coated microbubbles, with a diameter ranging from 1 to $10 \mu \mathrm{m}$, allowing them to reach the smallest capillaries [I]. The microbubbles scatter the ultrasound very effectively, since the gas core is compressible. Furthermore, enhanced scattering is caused by microbubble oscillations due to the applied sound field and the fact that the microbubbles display a resonance behavior at medical ultrasound frequencies. The microbubbles respond highly nonlinearly, giving rise to a further enhanced contrast-to-tissue ratio, when the linear response of the tissue is suppressed. Figure 1.2 shows an example of a rabbit kidney imaged before and after the injection of contrast agent microbubbles. The contrast-enhanced image clearly shows the delineation of the kidney and even the dynamical picture of the vascularization can be seen.
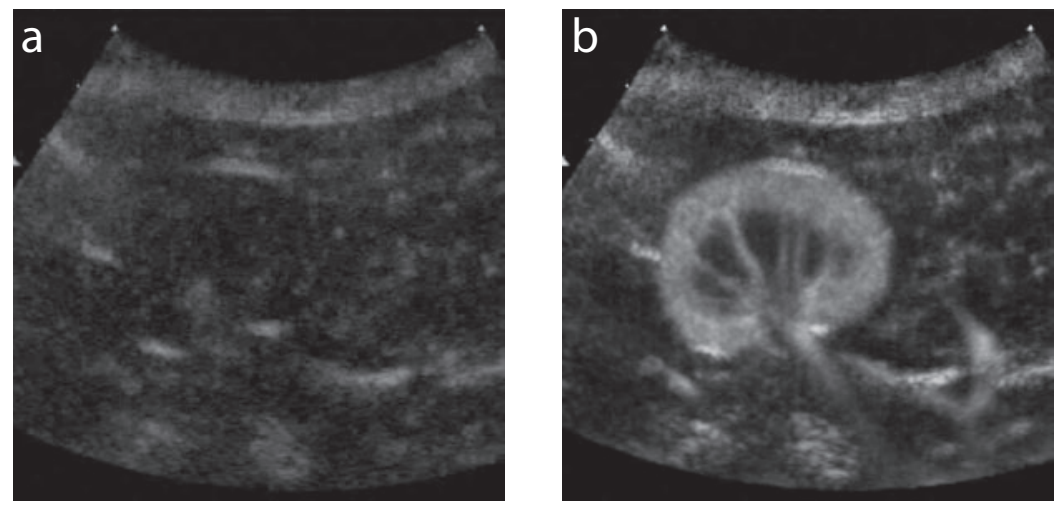

Figure 1.2: Ultrasound image of a kidney, before (a) and after (b) the injection of contrast agent microbubbles.

The interaction between the ultrasound and the microbubbles has been studied extensively over the last decades. By recording the acoustic backscatter of a microbubble, information about its response as a function of the ultrasound parameters, such as pressure and frequency, is obtained. The acoustic backscatter is a result of the microbubble oscillations and therefore an indirect measure of the bubble response. Optical microscopy is required to fully capture the microbubble behavior with sufficient spatial resolution. However, the nanoseconds timescales at which the oscillations take place, make time-resolved 


\subsection{THERAPEUTIC APPLICATIONS OF CONTRAST AGENTS}

optical imaging extremely challenging.

Only a few cameras available today are capable of achieving frame rates exceeding one million frames per second (Mfps). Nevertheless many new insights of microbubble dynamics have been gained in the last decade due to ultrahigh-speed imaging. The visualization of, for instance, "compression-only" behavior [2], nonspherical oscillations [3] or the interaction and clustering of multiple bubbles [4] would not have been possible without this imaging technique. Furthermore, the bubble radius is a key parameter in theoretical models that describe coated microbubble dynamics. Knowledge of the bubble behavior allows for adaptations of the applied ultrasound parameters and bubble composition to improve the contrast-to-tissue ratio and further enhance medical ultrasound diagnostics.

\subsection{Therapeutic applications of contrast agents}

Since several years, contrast agent microbubbles are being investigated to serve as therapeutic agents [5]. As a first step, functionalized microbubbles have been developed for molecular imaging [6]. By adding targeting ligands to the bubble surface, sites of inflammation or tumorous tissue can be detected using ultrasound. Secondly, microbubbles can be loaded with drugs to serve as drug carriers for local intravenous drug delivery; various configurations have been reported in literature [7, 8]. Drugs can be coupled directly onto the bubble surface or the drugs are first encapsulated and then linked to the bubble wall. Also, hard-shelled contrast agents microcapsules have been developed that carry a therapeutic payload in the core of the bubble.

Drug-loaded microbubbles are designed both for diagnostic ultrasound imaging at low acoustic pressures and local drug release upon disruption at high acoustic pressures. The release can be contained to within a small region through local ultrasound exposure, to reduce the systemic exposure to possibly toxic substances. Currently the mechanisms of drug release and that of the subsequent uptake, and the drug delivery efficacy are being investigated. The preliminary results are promising [9-12], and the ongoing research is now ported to the preclinical phase.

To study the ultrasound-triggered release and uptake of drugs using microbubbles, again, direct visualization of the process is preferred. As the microbubble oscillations take place on a nanoseconds timescale, the subsequent release is assumed to take place at a similar timescale. However, the objects of interest are at least an order of magnitude smaller, ranging from several hundreds of nanometers for drug-carrying vehicles, down to a nanometer for (a dispersion of) single molecules. Transmitted light microscopy does not offer the resolving 
power required for such small objects. Furthermore, in case of the instantaneous release of the contents of a drug-filled microbubble, the contrast between the released suspension and the surrounding medium is too low to distinguish the two flows, let alone in an in vivo experiment. Therefore fluorescence microscopy is required to visualize drug release from a microbubble. Fluorescently labeled molecules or vesicles yield a very high contrast, even in vivo, and transfection into cells can be visualized and nanometer-sized particles can be traced using fluorescence microscopy. In figure [1.3], the difference between transmitted light microscopy and fluorescence microscopy is illustrated. The fluorescent dye loaded onto the microbubble, depicted in figure $1.3 \mathrm{l} a$ and $b$, is only visible on the bubble surface in the fluorescence image. Figure $[1.3 \mathrm{c}$ and $d$ show a human endothelial cell and a human astrocyte, respectively. The astrocyte was stained with fluorescent markers that bind specifically to proteins (red) and DNA (blue) in the cell. The fluorescent staining gives additional information about e.g. the viability of the cell, which cannot be obtained from the transmitted light images directly.
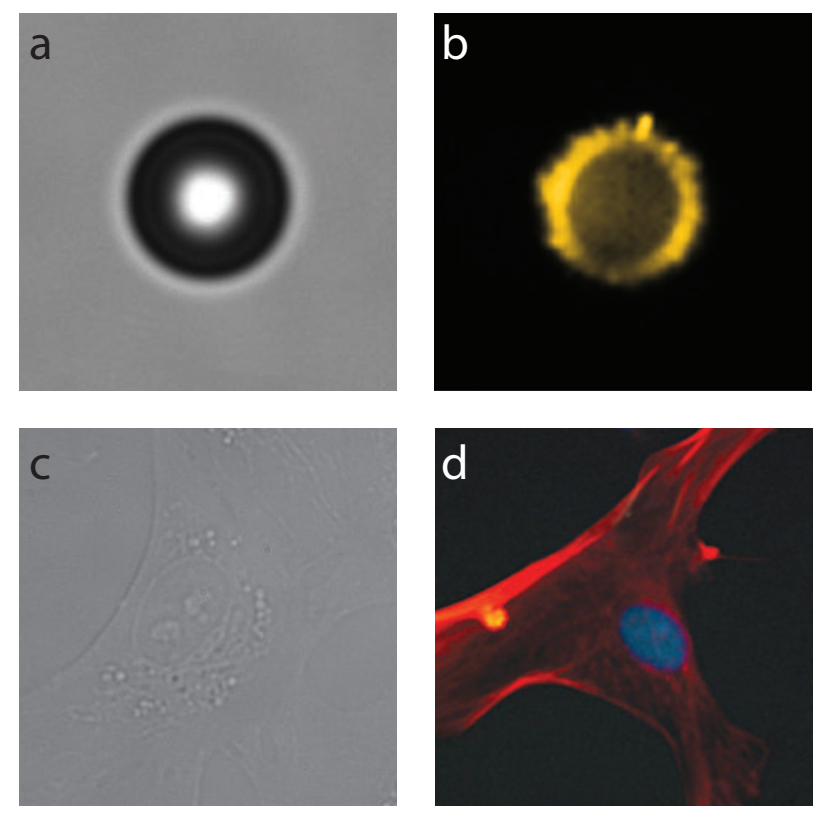

Figure 1.3: Transmitted light microscopy $(a, c)$ versus fluorescence microscopy $(b, d)$. The drugs attached to the surface of a microbubble $(a, b)$ were fluorescently labeled. c) A human endothelial cell in bright field. d) A human astrocyte with fluorescently labeled proteins (red) and DNA (blue). 


\subsection{Ultra-high-speed fluorescence imaging}

In ultra-high-speed imaging of microbubbles, it is already a challenge to obtain sufficient illumination levels to allow for nanoseconds exposure times. Specialized xenon strobes are employed to provide the bright field illumination and even then high gain settings on the image sensors are often required. Nonetheless, an increase in illumination intensity will yield more photons at the image sensor. In fluorescence imaging, collecting enough fluorescence emission photons is more complicated. First of all, only a small part of the excitation photons are converted into usable emission photons, since not all excitation light will be absorbed, the direction of the emission light is omnidirectional and the fluorescent molecules have a limited quantum yield. Secondly, increasing the excitation intensity does not necessarily yield more fluorescence emission as saturation of the excited states of the molecules occurs. In addition, the number of fluorescent molecules is finite due to the (sub)micron dimensions.

One method to facilitate fluorescence imaging on a nanoseconds timescale is to use laser-induced fluorescence (LIF). Very high excitation intensities ( $\mathrm{TW} / \mathrm{cm}^{2}$ ) are obtained with Q-switched Nd:YAG lasers generating laser pulses of several nanoseconds in length and pulse energies ranging van microjoules up to several joules. These lasers are commonly employed in a dual-cavity configuration for particle image velocimetry (PIV) and particle tracking velocimetry (PTV) using fluorescent tracer particles to visualize flows [13]. A drawback of pulsed laser systems is their 'limited' pulse repetition rate, which is typically restricted to $200 \mathrm{kHz}$. Time-resolved fluorescence imaging at $1 \mathrm{Mfps}$ then requires the use of a continuous wave (CW) laser. CW lasers, such as Argon-ion lasers, have been used in high-speed fluorescence imaging applications, however, maximum reported imaging frame rates are on the order of $10 \mathrm{kfps}$ [14].

\subsection{Guide through the chapters}

In this thesis we pioneer the use of continuous laser excitation for ultra-highspeed fluorescence imaging at frame rates ranging from $1 \mathrm{kfps}$ up to $25 \mathrm{Mfps}$. This imaging technique is applied to study the mechanisms of ultrasoundtriggered local drug delivery using microbubbles. The imaging system that was developed for this purpose is described in chapter 2, elucidating the essential design steps taken to achieve this setup.

In addition to the application of fluorescence imaging to the study of ultrasound-triggered drug delivery, it can also aid the research of microbubble dynamics. Chapter 3 deals with the difficulties that arise when microbubbles are imaged using transmitted light microscopy, the most typical optical method 
applied in current microbubble studies. By using fluorescence imaging, the absolute size of the bubble can be determined at higher precision. This leads to more accurate input data for theoretical models.

The process of ultrasound-triggered intravenous drug delivery can be split into two parts: the release from a drug-loaded microbubble and the subsequent uptake by a vascular endothelial cell. In chapter 4 the first part is addressed, where ultra-high-speed fluorescence recordings of the controlled release from liposome-loaded microbubbles are presented. Likewise, the enhanced uptake of endothelial cells induced by microbubble oscillations is captured, as discussed in chapter 5 on sonoporation. Although both processes are caused by microbubble oscillations with similar acoustic driving frequencies, the resulting release and uptake take place at timescales that differ by several orders of magnitude.

In chapter 6 and 7 two novel designs of drug-carrying contrast agent microcapsules are presented. The first design consists of a multimodal contrast agent for photoacoustic and ultrasound imaging. The second therapeutic agent can be employed as a two-step drug delivery system. Both microcapsule designs are characterized experimentally. Their response to laser light and ultrasound, respectively, is compared to new physical models describing the activation dynamics of these microcapsules, which were found to be in very good agreement. 


\subsection{GUIDE THROUGH THE CHAPTERS}




\section{2 \\ Brandaris 128 ultra-high-speed imaging facility: 10 years of operation, updates and enhanced features}

The Brandaris 128 ultra-high-speed imaging facility has been updated over the last 10 years through modifications made to the camera's hardware and software. At its introduction the camera was able to record 6 sequences of 128 images $(500 \times 292$ pixels $)$ at a maximum frame rate of $25 \mathrm{Mfps}$. The segmentation of the camera was revised to allow for a subdivision of the in total 768 images from the 128 sensors into arbitrary segments with an intersegment time of $17 \mu \mathrm{s}$. Furthermore, a region of interest can be selected to increase the number of recordings within a single run of the camera up to 125 , resulting in a total image count of 16000 . By extending the imaging system with a gated CW laser, time-resolved ultra-high-speed fluorescence imaging of microscopic objects has now been enabled. 


\subsection{INTRODUCTION}

\subsection{Introduction}

Ten years after its introduction, the Brandaris 128 ultra high-speed imaging facility [15] is still the fastest digital high-speed camera in the world, capable of recording more than 100 consecutive frames. Employing a rotating mirror, mounted on a helium-driven turbine, a maximum frame rate of 25 million frames per second (Mfps) is achieved. Other cameras, equipped with multiple synchronized sensors, are able to reach higher frame rates up to $200 \mathrm{Mfps}$, using specialized image intensifiers that can be gated down to $5 \mathrm{~ns}$. However, the number of frames within a single recording is limited to 16 in those cameras.

The Brandaris 128, shown in figure $2.7 \mathrm{l}$, is based on the camera frame of the Cordin 119 (Cordin Scientific Imaging, Salt Lake City, Utah). The rotating mirror camera design allows for a high frame rate and high number of frames, while the CCD sensors offer superior recording sensitivity and flexibility. Various lenses can be mounted to suit the experimental dimensions, ranging from microscope objectives to SLR-camera objectives. The primary image of an object is projected onto the rotating mirror prism by two relay lenses. The three-sided mirror is mounted onto a helium-driven turbine to redirect the image through the lens bank onto the CCD sensors.

The optical configuration is schematically depicted in figure 2.2la. The Miller principle for high-speed cinematography [16] was applied to obtain stationary images while the mirror sweeps the light beam across the 128 lens pairs in the lens bank, which refocus the image on 128 highly-sensitive, un-intensified CCD image sensors $(500 \times 292$ pixels $)$. The field lens and aperture are used to match the numerical aperture (NA) of the light beam to the NA of the lens pairs, thereby setting the exposure time per channel. As the mirror rotates, the image formed on a single detector changes slightly. The resulting blur, called drag, is minimized by projecting the primary image as close as possible onto the apparent axis of rotation of the mirror [17]. A maximum drag of $7.5 \mu \mathrm{m}$ is produced by the optical configuration and $50 \%$ of the channels suffer a drag of less than $4 \mu \mathrm{m}$, which is smaller than the pixel height of the CCD's $(12 \mu \mathrm{m})$.

A mass flow controller (MFC) (F-206Al, Bronkhorst, Ruurlo, The Netherlands) was installed to regulate the flow of helium to the turbine. Before an experiment is performed the camera case is pre-filled with helium to reduce the viscous drag. The maximum turbine speed of $20000 \mathrm{rps}$ is achieved at a flow rate of $2500 \mathrm{~L} / \mathrm{min}$ and results in a frame rate of $25 \mathrm{Mfps}$. 


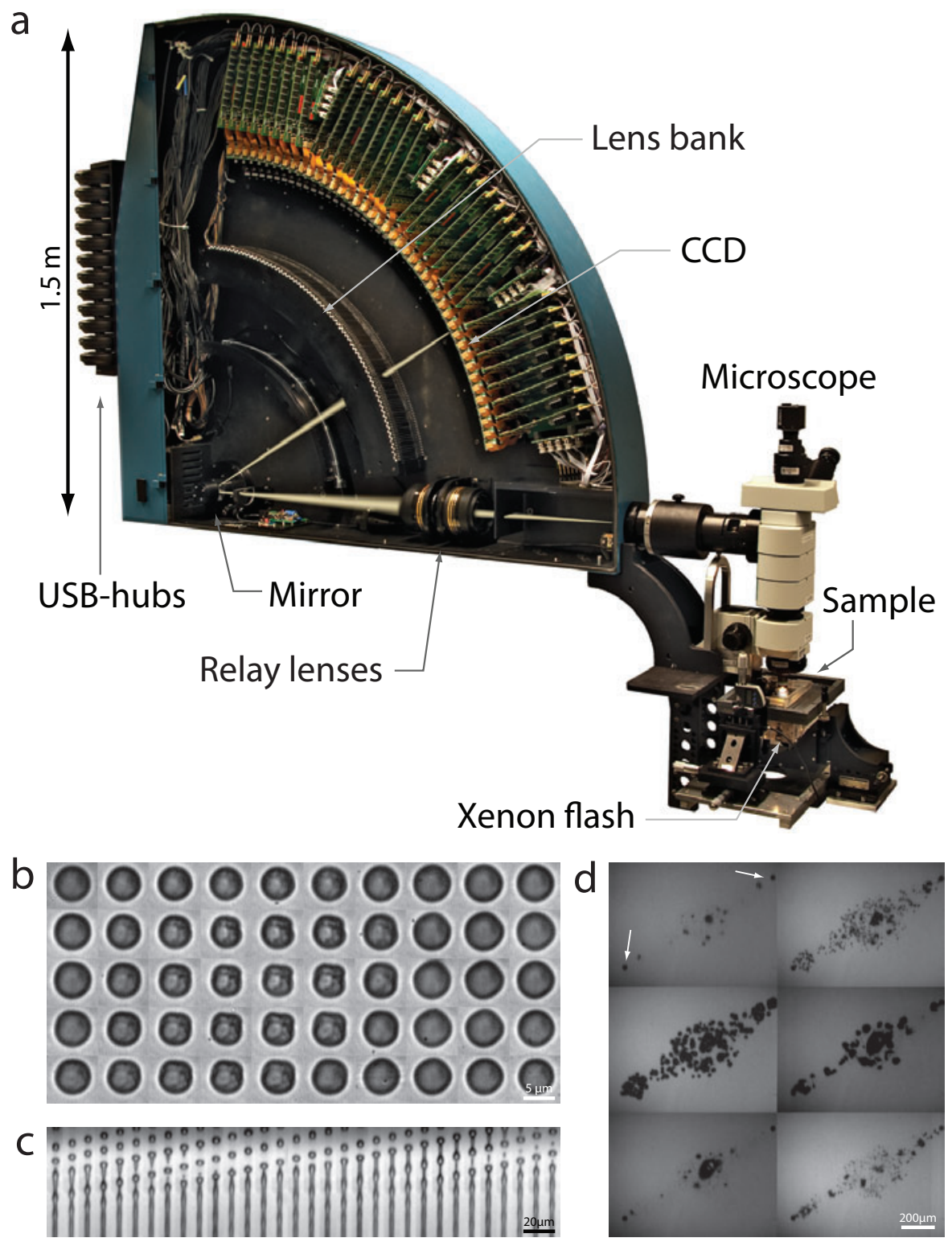

Figure 2.1: a) The Brandaris 128 ultra high-speed imaging facility. b) Ultrasound contrast agent showing buckling behavior recorded at $14.5 \mathrm{Mfps}$. c) Breakup of a $1.25 \mu \mathrm{m}$ radius liquid jet into microdroplets, visualized with an interframe time of 73 ns. d) Cleaning of a silicon wafer by the growth and collapse of a cloud of cavitation bubbles located in between two micropits (white arrows), recorded at $5.4 \mathrm{Mfps}$. 


\subsection{INTRODUCTION}

A custom designed $C C D$ controller card $\left(C^{3}\right)$ coordinates the image capture process for every four CCD detectors. Several microseconds before exposure of the first channel, all CCD's are flushed to drain the accumulated charge in the photosensitive cells. After exposure of the last channel a charge transfer shifts the photoelectrons into the transport channels of the CCD's, preventing multiple exposure of the CCD's. After the charge transfer, all pixels are read out for analogue-to-digital conversion and the image data is stored in the onboard RAM of the CCD's. Flush and transfer only take several microseconds to perform, whereas readout takes $80 \mathrm{~ms}$ to finish. When the readout is completed another recording can be made. The onboard RAM of the CCD's limits the number of recordings within one experiment to 6 , leading to a total number of 768 frames. After the final readout, all image data is transferred via USB-hubs to a PC for data storage and analysis. Since this takes only several seconds, many experiments can be performed in a short time frame.

A single experiment performed with the Brandaris 128 in default mode consists of 6 recordings, using all 128 frames for each recording, as depicted in fig-

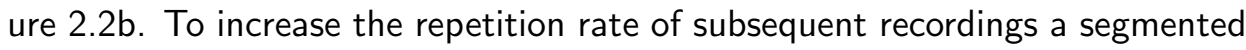
mode was devised, as shown in figure $\overline{22 c}$ c. The total number of 128 frames can be divided into 2 segments of 64 frames or 4 segments of 32 frames. After exposure of the first segment, only the charge of the first segment is transferred into the transport channels of the corresponding CCD's. During a subsequent mirror period, all CCD's are flushed again and after exposure the second segment undergoes charge transfer. The data in the transport channels of the first segment is unaffected by the additional exposures, flushes and charge transfers. After all charge transfer is completed for the last segment, readout can be initiated for all channels. In theory an inter-segment time as short as the mirror period divided by the number of mirror faces can be achieved, resulting in $17 \mu \mathrm{s}$ for a three-sided mirror.

The Brandaris 128 has been dedicated predominantly to the study of medical ultrasound contrast agents. These gas-filled, micrometer-sized, coated bubbles are very efficient scatterers of ultrasound and are used in organ perfusion imaging. In order to understand the interaction of the bubbles and ultrasound which allows us to improve their performance, detailed insight of microbubble dynamics is crucial. Since the applied ultrasound frequencies and corresponding microbubble oscillations are in the order of $1-5 \mathrm{MHz}$, ultra high-speed imaging at a frame rate exceeding $10 \mathrm{Mfps}$ is required.

The microbubbles are resonators to the applied sound field, hence their response is a function of both pressure and frequency. By systematically varying the ultrasound parameters and recording the microbubbles oscillations, the response of single phospholipid-coated microbubbles is now well understood. A 

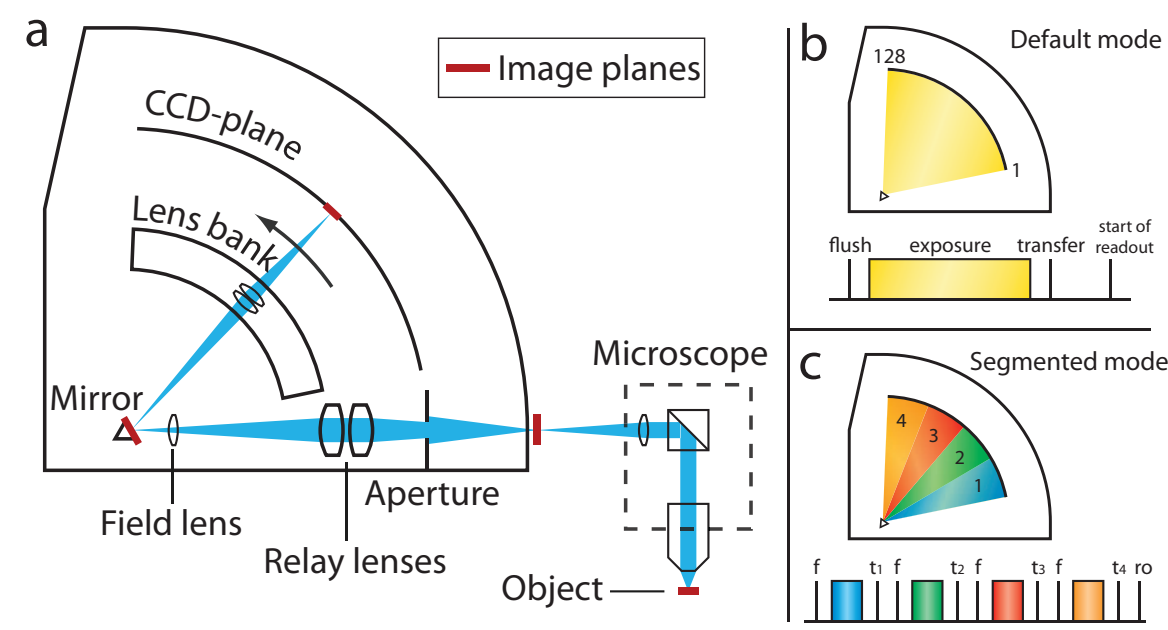

Figure 2.2: a) Schematic representation of the optical configuration of a rotating mirror framing camera. b) Exposure diagram for a recording in default mode. c) Exposure diagram for a recording in segmented mode.

major contribution was made by Van der Meer et al. [18] in 2007 when the camera was operated in segmented mode. This allowed for the recording of 12-24 sequences in a single experiment to fully capture a bubble's resonance curve within a single run of the camera by changing the insonation frequency in each segment, as shown in figure $[2.3$. This method was termed bubble spectroscopy, since a single experiment revealed the characteristics of a single bubble for a scan of a chosen parameter. Other studies visualized for the first time phenomena such as shell buckling [I9], "compression-only" behavior [2], "thresholding" behavior [20], nonspherical oscillations [3], and subharmonic oscillations [2I].

Enhanced functionality was achieved by combining the camera with optical tweezers to perform 3D micro-manipulation of microbubbles [22]. This enabled the comparison of bubble oscillations near a wall and at a distance from the wall within a single experiment, by applying a Laguerre-Gaussian laser beam to control the vertical position of a microbubble. In the same way, the influence of neighboring bubbles was studied by controlled variation of the distance between two bubbles.

New insights into the physical mechanisms of ultrasound-triggered local drug delivery were found by studying the release and uptake of drugs by cultured cells. Recordings of a hard-shelled, oil-filled agent showed the disruption of the polymer wall and subsequent release of the contents [23]. Van Wamel et al. studied the interaction of a microbubble and an endothelial cell [24]. The high-speed recordings revealed the mechanical forces a bubble can exert on 


\subsection{INTRODUCTION}
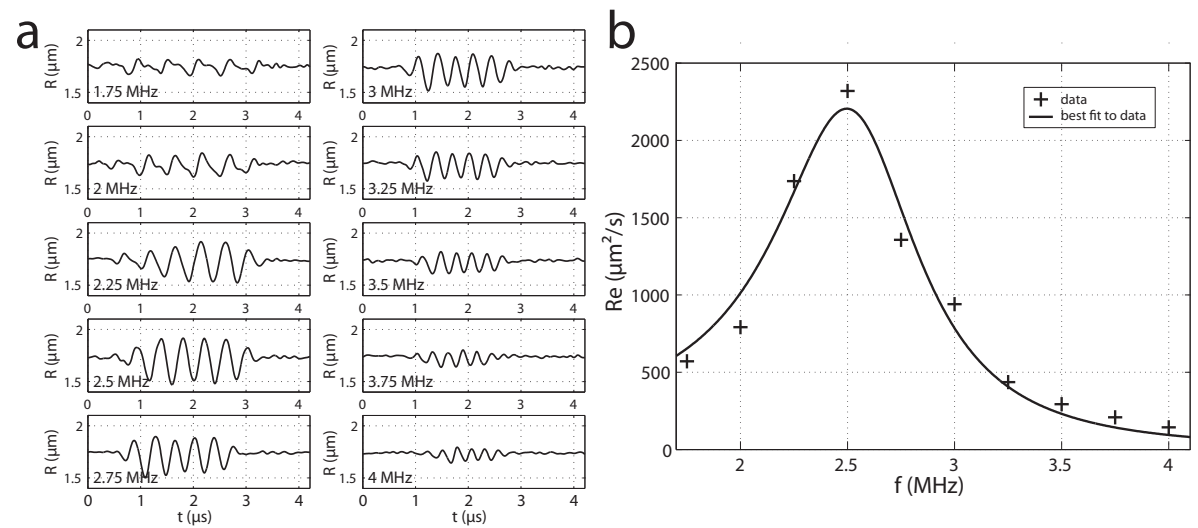

Figure 2.3: Microbubble spectroscopy. a) Radius-time curves of a microbubble for different excitation frequencies, recorded in a single run of the Brandaris 128. b) Resonance curve obtained from the radius-time curves of a single run.

the cell membrane, by visualizing the cell deformation following microbubble oscillations. In combination with simultaneous fluorescence recordings, an enhanced permeability of the cell membrane, caused by acoustic streaming, was confirmed.

The Brandaris 128 has also been used to study other fast phenomena that occur on a nanoseconds timescale, such as droplet formation in piezo inkjet printing and pulmonary drug delivery [25], and bubble-induced cavitation for the cleaning of silicon wafer surfaces [2b]. Figure $[2.] \mathrm{b}-\mathrm{d}$ shows several examples of observed phenomena.

\subsubsection{System limitations}

Despite the numerous novel scientific results obtained with the Brandaris 128, the camera still has its limitations, the first being the recording speed. An increase in frame rate would open up a range of possible applications. Even a 'minor' improvement of a factor 2 (50 Mfps) would enable the study of microbubbles excited by ultrasound frequencies in the order of $20 \mathrm{MHz}$. These frequencies are being used for high-resolution intravascular ultrasound imaging. At this point, increasing the frame rate can only be achieved through major alterations to the design of the camera, which, in terms of costs, would be in the same order of a full redesign of the system.

Secondly, the original timing controller of the Brandaris 128 that generated the triggers for the ultrasound, the illumination and the flush and transfer of the CCD's, was based on manual switches and timer/counter equipment. It 
required at least two people to operate and the response time was approximately 2 seconds. To ensure accurate timing, the turbine had to be run for an extended period of time until the speed had stabilized.

Each channel has the capacity to store 6 images in its onboard RAM. It enables the user to perform 6 recordings in less than a second, which is an advantage over other high-speed cameras that can typically only record 1 to sometimes 2 images per sensor. Nevertheless, even 6 recordings are insufficient to capture the dynamics of a microbubble for a small set of three different pressures at three different insonation frequencies. Full characterization then requires multiple experiments taking several minutes to complete in which the properties of the microbubbles can change significantly. This problem can be addressed by using 24 recordings in the segmented mode, however, this limits the number of frames per recording to 32 .

Aside from the reduced number of frames per recording, when it was first introduced the segmented mode was also limited in its inter-segment time. Since no actual read-out of the sensors is required until all segments are illuminated, the theoretical time between subsequent segments is the revolution period of the mirror divided by the number of mirror faces. However, these specifications were not met, resulting in a time between segments equal to the time between full sequence recordings, i.e. $80 \mathrm{~ms}$. Furthermore, the number of segments was limited to four.

Finally, for imaging at a micrometer length scale, the typical technique used is bright field microscopy using back illumination, since it has the highest yield in light intensity. At an exposure time of $40 \mathrm{~ns}$ and magnifications up to 200 times, every photon counts. By using a special Xenon strobe, bright field sequences can be recorded up to the limiting frame rate of the camera. However, when the sample does not allow for bright field imaging and reflected instead of transmitted light is required, for instance using dark field illumination, the signal-to-noise ratio (SNR) of the images is decreased significantly, due to the lowered photon flux reflected by the sample. Using a standard fluorescence microscope, an exposure time in the order of tens of milliseconds is required to visualize a fluorescently labeled microbubble. When fluorescence imaging is required for particle tracking or to visualize drug release or uptake from a microbubble at a (sub)microsecond timescale, coupling the camera to a fluorescence microscope setup simply does not yield enough photons.

\subsubsection{Enhanced features}

To overcome several of the aforementioned limitations, adaptations have been made to both the hardware and software of the camera in the last decade. First, 


\subsection{INTRODUCTION}

a dedicated timing controller was developed for generating "real-time" trigger signals for illumination, ultrasound and the CCD's. By constantly monitoring the mirror speed and adapting the pre-trigger delays for various devices, a timing accuracy of less than $40 \mathrm{~ns}$, i.e. the interframe time at the maximum frame rate, was achieved. As variations in the mirror speed are now accounted for continuously, the user does not have to wait for the turbine to run at a stable speed before starting the actual experiment, thereby reducing the time required to reach the desired frame rate and limiting the wear of the turbine. Furthermore, the system was designed to be operated by autonomous triggering at a preset frame rate.

A second improvement was made with the goal to increase the number of recordings within a single run, which is limited by the storage capacity of the CCD's. In many experiments, only a single bubble or an event that is small compared to the full image frame is of interest. Nevertheless a full frame will be stored in the onboard RAM. By letting the user select a region of interest on the CCD's, the image size can be decreased, overcoming the limit of 6 images per channel. This drastically increases the number of recordings within one experiment. It also decreases the number of times the turbine has to be run when a parametric study is being conducted, again limiting the wear of the turbine.

Thirdly, software changes were made to use the full capacity of the segmented mode, decreasing the inter-segment time from $80 \mathrm{~ms}$ to $17 \mu \mathrm{s}$. Furthermore the number of segments, the number of frames per segment, and the location of the segments can now be chosen arbitrarily.

Finally, the camera system was adapted to be able to perform fluorescence microscopy on a nanoseconds timescale. To assess the feasibility of a highspeed fluorescence imaging system based on the Brandaris 128, an analysis was made, taking into account all parts that contribute to obtain an acceptable SNR at $25 \mathrm{Mfps}$ : the image sensor, the imaging optics, the fluorescent dye and the excitation light source. This can be described by the following equation:

$$
S N R \propto Q E_{\mathrm{CCD}} \times N A \times \Phi_{\text {photon }},
$$

where $S N R$ is the signal-to-noise ratio, $Q E_{\mathrm{CCD}}$ is the quantum efficiency of the image sensor, NA is the effective numerical aperture of the optical system and $\Phi_{\text {photon }}$ is the photon flux generated by the fluorescent object. Improving the image sensor would mean replacement of the current Sony ICX-055AL chips; a chip that was chosen specifically for its high sensitivity. The intrinsic sensitivity of CCD-sensors has not increased much since 2000. Back-illuminated CCD's will have a higher quantum efficiency, but this would increase the sensitivity only by a factor of 2 . The use of image intensifiers or electron multiplication 
applied in EMCCD's will increase the sensitivity by one or perhaps two orders of magnitude, however, both techniques result in a large image sensor, whereas the available space for each channel is limited, as shown by Chin et al. [15]. Also, the first technique introduces very high noise levels and the second technique requires extensive cooling, which adds to the already increased size of each image sensor. Furthermore, enormous costs would be involved in the replacement of the sensors and controller cards, and the required redesign of the camera interior. The numerical aperture of the microscope system is already optimized by the use of high-NA water immersion objectives. The camera design itself limits the NA through the separation distance between two consecutive channels. At the highest magnification, the width of the lenses that produce the images on the CCD's exactly matches the NA of the beam exiting the microscope, leaving no room for improvement. This implies that the increase in sensitivity must come from the generated fluorescence emission.

The photon flux coming from the sample depends on the excited state kinetics of the fluorescent molecules, assuming a steady state is reached and photobleaching is neglected:

$$
\Phi_{\text {photon }}=\frac{\phi}{\tau_{s}} n N_{s}
$$

where $\phi$ is the quantum yield, with a maximum of 1 . Many commercially available dyes have an efficiency of approximately $70 \%$ or higher. The fluorescence lifetime $\tau_{\mathrm{s}}$ is in the order of 0.5 to 20 nanoseconds, showing little margin for enhancement. The number of molecules $n$ depends on the concentration of fluorophore and the area or volume available for labeling. Only the fraction of molecules residing in the excited state $N_{\mathrm{s}}$ can be exploited for increased fluorescence yield, as the other parameters are largely determined by the type of dye and concentration that can be used, depending on the object that needs to be labeled.

$N_{\mathrm{s}}$ determines the amount of fluorescence emission and depends on the rate constant of the excited state $k_{\mathrm{s}}\left(k_{\mathrm{s}}=\tau_{\mathrm{s}}^{-1}\right)$ and the excitation rate $k_{\mathrm{a}}$ of the molecules:

$$
\begin{gathered}
N_{\mathrm{s}}=\frac{k_{\mathrm{a}}}{k_{\mathrm{s}}+2 k_{\mathrm{a}}}, \\
k_{\mathrm{a}}=\frac{\sigma \lambda}{h c} I,
\end{gathered}
$$

where $\sigma$ is the absorption cross section of a molecule, $\lambda$ is the excitation wavelength, $h$ is Planck's constant, $c$ is the speed of light and $I$ is the excitation intensity delivered by the light source. At low excitation intensities $\left(k_{\mathrm{s}} \gg k_{\mathrm{a}}\right)$ 


\subsection{INTRODUCTION}

the photon flux is proportional to the intensity of the excitation light, which is the case for a mercury arc burner or high-intensity LED light source:

$$
\Phi_{\text {photon }}=\phi \frac{\sigma \lambda}{h c} I n .
$$

This suggests that increasing the excitation intensity by several orders of magnitude, for instance through the use of a laser, should be sufficient to achieve the required improvement to go from a milliseconds exposure time down to a nanoseconds exposure time. However, at high excitation intensities $\left(k_{\mathrm{s}} \ll k_{\mathrm{a}}\right)$, saturation occurs and the photon flux becomes independent of the excitation intensity:

$$
\Phi_{\text {photon }}=\frac{\phi}{2 \tau_{s}} n .
$$

This result shows that when the excitation intensity is sufficient to cause saturation, the fluorescence signal is almost solely determined by the number of dye molecules. For a phospholipid-coated microbubble labeled with a fluorescent dye such as rhodamine or Dil-C18, the total number of fluorescent molecules is limited by the surface area of the bubble. Nevertheless preliminary experiments using laser-induced fluorescence showed that fluorescence imaging of microbubbles at frame rates exceeding $1 \mathrm{Mfps}$ was feasible. Figure 2.4 shows a phospholipid-coated microbubble and a pair of oil-filled polymer microcapsules. Both were imaged with the Brandaris 128 with an exposure time of 500 and $55 \mathrm{~ns}$, respectively. The high loading capacity of the polymeric capsules allowed for fluorescence imaging up to the maximum frame rate of the camera. The microbubbles, with a much less efficient loading of fluorescent dye molecules, could be imaged up to $2 \mathrm{Mfps}$.
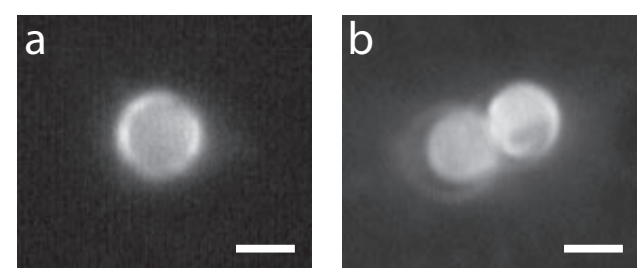

Figure 2.4: Fluorescence images recorded with the Brandaris 128 of a) a fluorescently labeled phospholipid-coated microbubble (500 ns exposure time) and b) a pair of fluorescently labeled, oil-filled polymeric microcapsules ( $55 \mathrm{~ns}$ exposure time). Scale bars represent $5 \mu \mathrm{m}$. The high pay-load of the polymeric capsules allowed for fluorescence imaging up to the maximum frame rate of the camera. 


\subsection{System description}

\subsubsection{Dedicated timing controller}

The trigger system consists of a dedicated $\mathrm{PC}$ with a $\mathrm{PCl}$-based timer/counter with IO-interfacing (NI 6602, National Instruments, Austin, Texas) and a PClbased programmable delay board (BME sG02p, Bergmann Messgeräte Entwicklung KG, Murnau, Germany). The input for the system is the mirror pulse, produced by an infrared laser/photodiode pair, mounted at $30^{\circ}$ below the optical axis, providing accurate measurement of the instantaneous mirror rotation rate and mirror position. The controlling software is written in C. It records every mirror pulse interval and saves it for logging purposes. Every $4 \mathrm{~ms}$ the delays of the various trigger signals are adjusted for the running estimate of the mirror speed. Arming of the delay board is combined with an extra control signal such as a manually generated input signal or a preset frame rate.

The accuracy obtained with this system depends on two parts and can be calculated as follows. The first cause of a trigger inaccuracy is the read-out of the mirror pulse interval by the timer/counter. By averaging over tens of pulses, the accuracy is increased from $12.5 \mathrm{~ns}$ to a few nanoseconds.

The second cause is the change in frame rate, caused by variations in the turbine rotation speed. When the voltage to the MFC is set for a chosen frame rate, the speed will show an overshoot before reaching a stable value. To reduce the wear of the turbine, experiments are now performed around the overshoot, which results in an increased variation in the turbine speed during an experiment with a maximum of $15 \%$ per second, resulting in a variation of $0.06 \%$ within one loop of the timing controller. This accuracy also applies to the triggered signals, which, if kept below $50 \mu \mathrm{s}$, results in a timing error of less than $30 \mathrm{~ns}$. These numbers suggest a timing accuracy of less than one frame at the maximum frame rate and this conclusion has been confirmed by experiments.

\subsubsection{Region of interest mode}

Instead of using the full frame, a region of interest (ROI) can be selected if there is a need for recording more than 6 exposures using all 128 channels for each exposure. In the ROI mode the user has the option to select a subsection of the full frame as shown in figure 2.5. Because the image size is reduced, the number of images that can be stored per CCD in the onboard RAM is increased.

The horizontal position of the ROI can be selected from 11 pre-defined values and the vertical position is fixed around the center of the frame. The vertical symmetry was chosen because of limited resources of the field-programmable 


\subsection{SYSTEM DESCRIPTION}

gate array (FPGA) on the CCD-controller. Both the height and width of the $\mathrm{ROI}$ can be selected from 9 pre-defined values. The minimum size is $105 \times 78$ pixels and the maximum size is $500 \times 292$ pixels, which is equal to a full frame. Selecting the minimum size of the ROI results in a maximum of 125 exposures. In a typical experiment a $\mathrm{ROI}$ of $153 \times 114$ pixels results in 60 exposures within a single experiment. Each exposure requires $80 \mathrm{~ms}$ to be transferred to the onboard memory, leading to a total time of 4.8 seconds in which 60 unique experiments can be recorded.

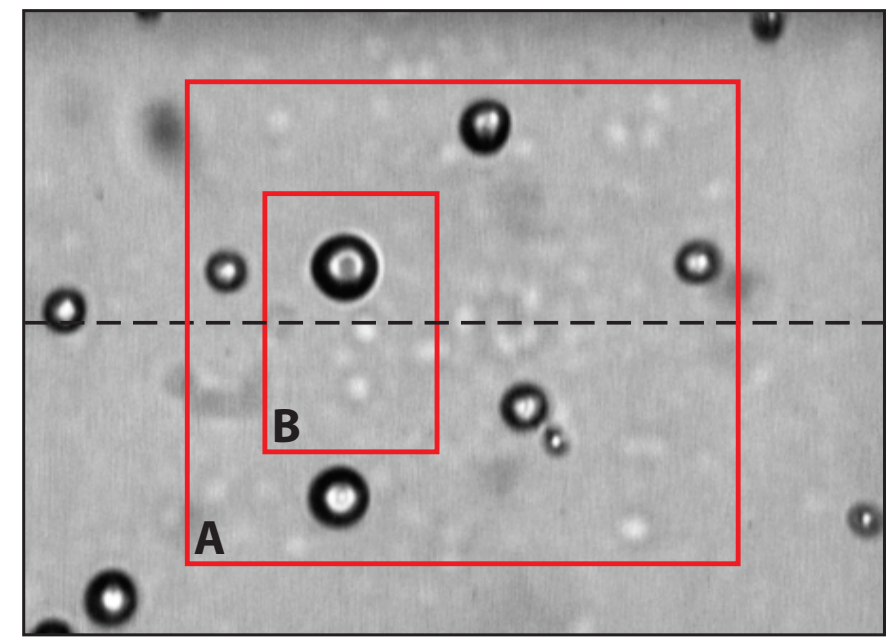

Figure 2.5: The region of interest mode lets the user select a region of the full frame to increase the number of full length exposures. The only constraint is that the $\mathrm{ROI}$ has to be vertically centered. The marked rectangle ' $A$ ' has a size of $320 \times 240$ pixels corresponding to 12 exposures. Rectangle ' $B$ ' has a size of $100 \times 120$ pixels corresponding to 72 exposures.

\subsubsection{Segmented mode}

The segmented mode was devised with the purpose to increase the number of recordings within one experiment and to have an inter-segment time in the order of microseconds. The latter requirement was not achieved yet during the introduction of the Brandars 128 because of previous limitations of the timing controller and the limited pulse length and pulse repetition frequency of the light source. Adaptations to the software that controls the triggering and to the light source have been made since then to enable subdivision of the 128 channels into arbitrarily chosen segments with a short inter-segment time.

The time between segments can be strongly reduced since the image data is 
transferred quickly from the sensor into the transport channel before a second exposure, where the data is unaffected by any additional exposures of the CCD. The read out process takes approximately $80 \mathrm{~ms}$, whereas transfer of the image data into the transport channel is completed within $10 \mu \mathrm{s}$. After the recording of the final segment, the image data from all channels is transferred to the RAM, and after $80 \mathrm{~ms}$ the first segment can be exposed again.

The user now has full control over the number of frames in each segment, the position of the frames and the time delay between the segments. Figure 26 shows a timing diagram in which the 6 exposures are depicted horizontally and the different segments within one recording are depicted vertically. The minimum size of a segment is now a single frame and the minimum intersegment time is equal to the rotation period of the mirror divided by the number of mirror faces. In case of a three-sided mirror rotating at its maximum speed of $20000 \mathrm{rps}$, the inter-segment time is $17 \mu \mathrm{s}$. Because each frame can be allocated separately to a specific segment, segments can contain several frames located randomly within the total of 128 channels.
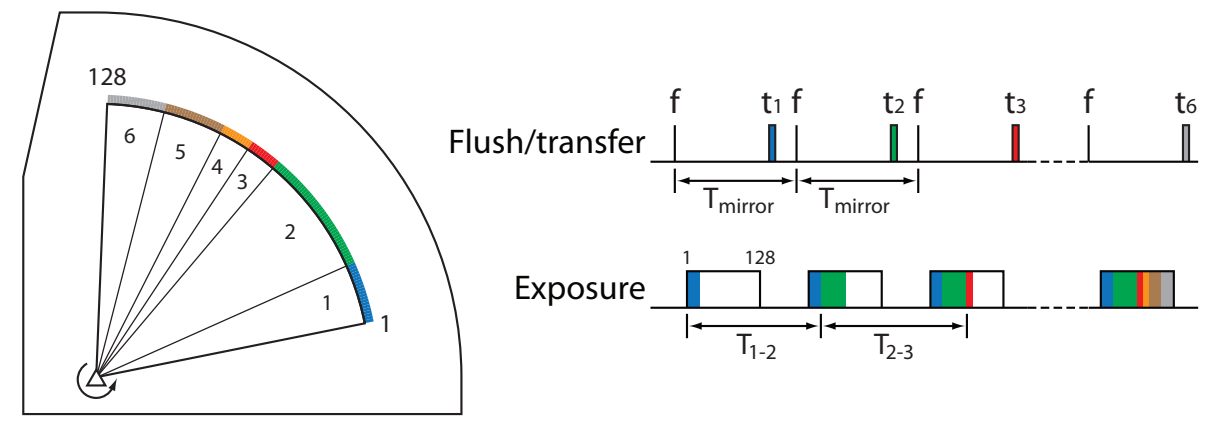

Figure 2.6: Schematic representation of the improved segmented mode. The 128 channels can be subdivided into segments with an arbitrary number of frames. The example shows 6 segments of different length. The timing of the flush and transfer triggers is matched to the mirror period $\left(T_{\text {mirror }}\right)$. The flush signals $(f)$ are sent to all channels, whereas the transfer signals $\left(t_{n}\right)$ are segment specific. The time difference between the start of two subsequent segments $\left(T_{n}-T_{n+1}\right)$ depends on their position.

In order to obtain inter-segment times on the order of microseconds, the timing sequence and the illumination had to be adjusted. A separate delay generator (Model 555, Berkeley Nucleonics Company) was used to control the triggering of the flush for the CCD's, the ultrasound and the illumination for each segment. The xenon flash light source used for bright field illumination has a maximum repetition rate of $15 \mathrm{~Hz}$, which is too slow to generate a separate pulse for each segment. On the other hand a single pulse, does not cover all segments, since the discharge pulse length is approximately $40-50 \mu$ s. Therefore 


\subsection{SYSTEM DESCRIPTION}

the electrical discharge circuit of the lamp was modified to have a discharge lasting up to $1 \mathrm{~ms}$, thereby allowing all segments to be illuminated by a single flash. The electrical capacitance was increased to maintain sufficient illumination levels, although it was observed that it reduced the lifetime of the light source.

\subsubsection{Ultra-high-speed fluorescence imaging setup}

Laser-induced fluorescence (LIF) is used in many research fields such as the visualization of turbulent mixing flows [27] or combustion processes [28], (micro) particle image velocimetry ( $\mu \mathrm{PIV})$ [I3], and molecular spectroscopy [29]. For measuring velocity fields often dual cavity Nd:YAG lasers provide the fluorescence excitation of micron-sized or even nanometer-sized tracer particles. Their typical pulse repetition frequency (PRF) is in the order of $10 \mathrm{~Hz}$, whereas $\mathrm{Nd}$ :YLF lasers can achieve PRFs up to $200 \mathrm{kHz}$. Nevertheless a separate cavity would be required for each individual frame to be able to do LIF at frame rates exceeding $1 \mathrm{Mfps}$ [30], rendering pulsed lasers unsuitable especially when taking into account the required power supply and cooling equipment.

Mode-locked lasers have fundamental repetition rates in the order of $100 \mathrm{MHz}$ and higher, fast enough to match the frame rates of the Brandaris 128. The high PRF results in multiple pulses per exposure at a timescale comparable to the fluorescence lifetime of a fluorescent molecule, resulting in a quasi-continuous illumination. However, compared to a continuous wave (CW) laser they are bigger, more expensive and require extensive cooling.

A CW laser does not reach the high peak intensity levels of a pulsed laser. It can utilize the full exposure time to excite the fluorescent molecules multiple times. Moreover, diode-pumped solid-state lasers come in a large variety of wavelengths, they have a small size, they do not require extensive cooling, and they are much more affordable than a pulsed laser system. Therefore a $5 \mathrm{Watt}$ DPSS CW laser (Cohlibri, Lightline, Germany) with a wavelength of $532 \mathrm{~nm}$ was chosen as the fluorescence excitation source.

Its small size allowed mounting underneath the camera, as depicted schematically in figure [2.7. The modular design of the microscope allowed for coupling of the laser light into the optical path of the microscope via a dichroic mirror. Two mirrors align the laser beam onto the beam expander. The beam is filtered after passing through a pinhole and is expanded to a width of $3 \mathrm{~mm}$. By adjusting the position of the plano-convex lens of the beam expander the divergence of the beam can be varied and the spot size at the sample level can be controlled. The dichroic mirror directs the laser light towards the imaging objective that focuses the excitation light onto the sample. A notch filter lo- 
cated above the dichroic mirror filters out any reflected excitation light from the fluorescence emission that has not been filtered out by the dichroic mirror. When recording in bright field with the fluorescence filters in place, $42 \%$ of the xenon illumination is filtered out by the dichroic mirror and notch filter, maintaining sufficient light levels for bright field imaging.

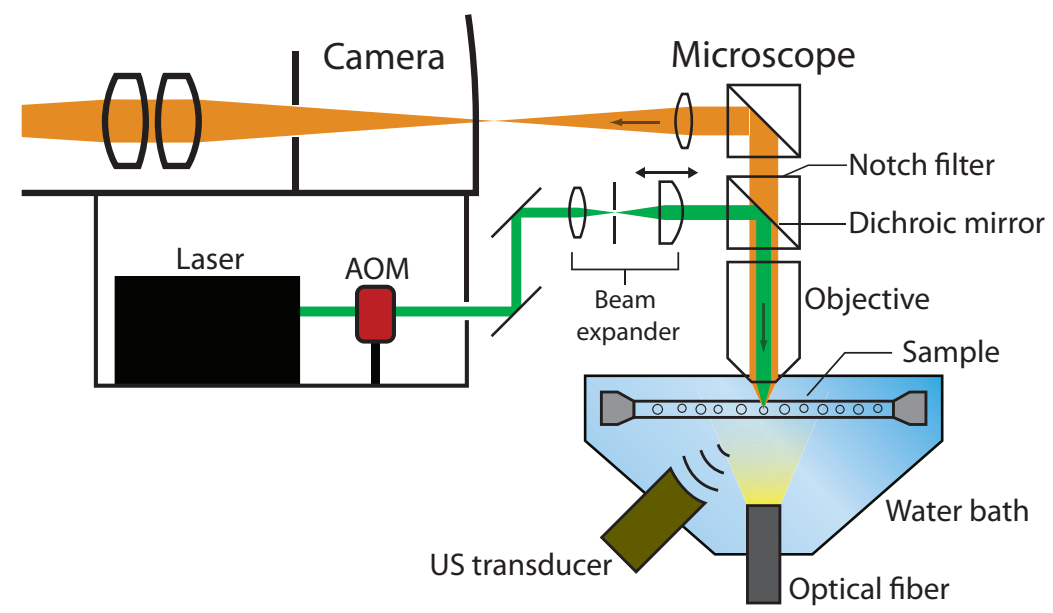

Figure 2.7: Schematic view of the experimental setup used for high-speed fluorescence recordings with the Brandaris 128 . The laser light is focused by the imaging objective onto the sample. The beam-expander filters the laser beam and allows for adjustment of the laser spot size.

Focusing of the laser light through the imaging objective is necessary to achieve sufficient excitation levels of the fluorescent dye molecules. Consequently the field of view is limited by the laser spot size. The smallest spot can be achieved by positioning the plano-convex lens such that the beam exiting the beam expander is parallel. A $60 \times$ water immersion objective with a numerical aperture of 0.9 , typically used for microbubble experiments, produces a spot with a full width at half maximum (FWHM) of $1.0 \mu \mathrm{m}$ and a maximum laser intensity of $72 \mathrm{MW} / \mathrm{cm}^{2}$. The spot diameter can be increased by making the laser beam divergent before it enters the objective. This greatly decreases the laser intensity as shown in figure 2.8. The optimal spot size depends on the observed sample and should be matched to the desired field of view.

Direct modulation of the voltage supplied to the laser head allows for a maximum switching rate of $1 \mathrm{kHz}$, which results in a rise time longer than the recording time of the Brandaris 128. The amount of photobleaching generated during the startup of the laser can be significant and at a millisecond timescale, unwanted thermal effects can start to play a role. Heating of the sample should be avoided especially when experiments are performed on biological samples. 

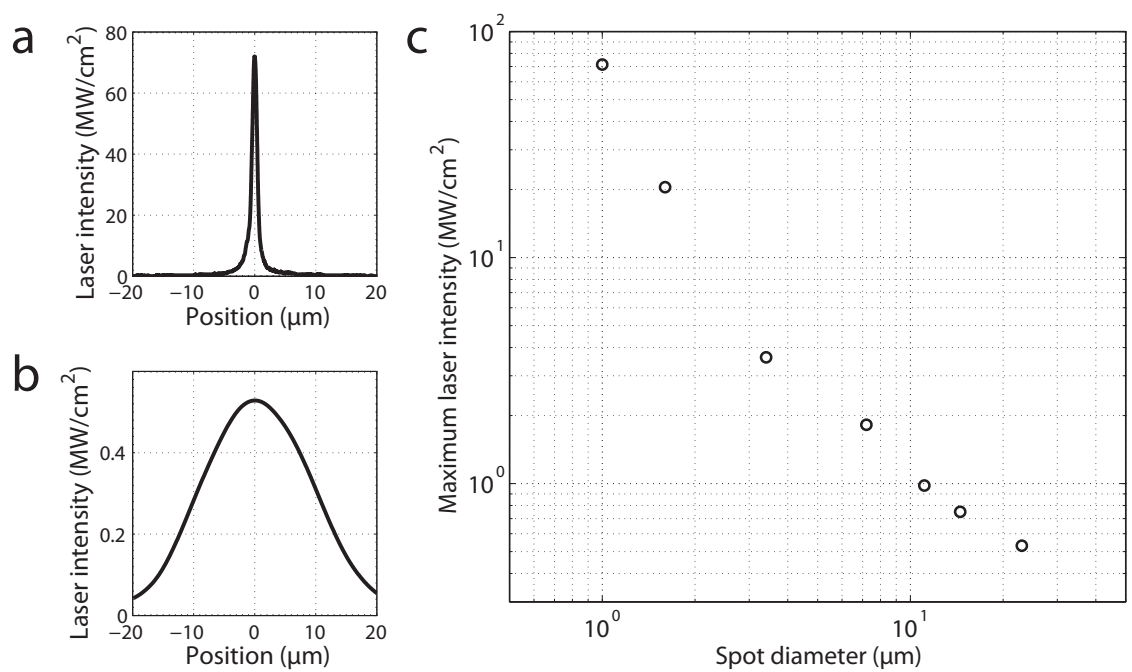

Figure 2.8: a) Laser beam profile for a spot with FWHM $1.0 \mu \mathrm{m}$ and b) $23 \mu \mathrm{m}$. c) The maximum excitation intensity as a function of laser spot size for a $60 \times$ objective with an NA of 0.9 .

To facilitate a faster rise time and shorter pulses, the laser was gated by an acousto-optic modulator (AOM) (AOTF.nC-VIS, AA optoelectronic, France) positioned in between the laser and the beam expander. The laser beam was sent through the AOM unfocused to minimize power loss, although this resulted in a slower switching speed. The light transmitting efficiency of the AOM is $92 \%$, resulting in a maximum power of $4.8 \mathrm{~W}$ for gate times of $5 \mu \mathrm{s}$ and longer, corresponding to a rise and fall time of $2.5 \mu \mathrm{s}$ as shown in figure 2.9 . Shorter gate times result in decreased laser power.
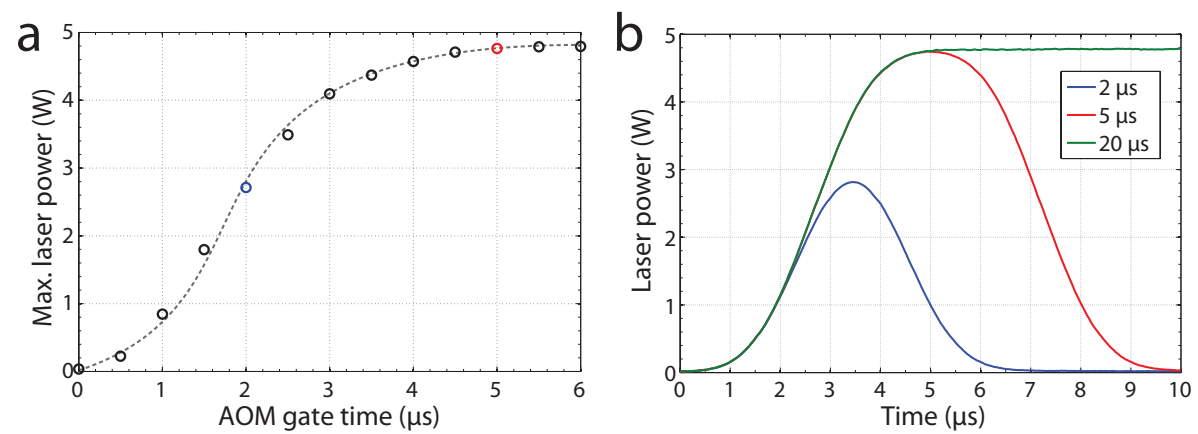

Figure 2.9: a) Maximum laser power for different gate times of the AOM. A maximum power of $4.8 \mathrm{~W}$ was obtained for gate times of $5 \mu$ s and longer. b) Pulse shape for three different gate times of the AOM. The rise and fall time of the AOM is $5 \mu$ s. 
With the fluorescence module in place, the Brandaris 128 configuration gives rise to three illumination options: bright field, fluorescence, and the combination of both illumination techniques. A digital delay generator (Model 575, Berkeley Nucleonics Corporation, San Rafael, CA) is used to trigger the laser and the $\mathrm{AOM}$, and the xenon strobe, giving full control over the illumination methods. For each individual recording within one experiment, the illumination method can be predefined. In the case of 6 full-frame recordings for instance, the first 3 can be captured in bright field and the final 3 in fluorescence. Other options are alternating bright field and fluorescence or even 6 combined recordings of which each recording is split into two virtual segments: the first 64 frames are recorded in bright field and the second 64 frames are recorded in fluorescence.

Experiments performed with the Brandaris 128 typically involve ultrasonic excitation of microbubbles or droplets. For this purpose a separate, preprogrammed ultrasound signal is uploaded for each recording to an arbitrary waveform generator (Model 8026, Tabor Electronics Ltd, Israel). A negative time delay is set in the timing controller to take into account travel time of the ultrasound. Figure 2.10 shows the timing diagram for two consecutive recordings within one experiment, the first being a bright field recording and the second being a fluorescence recording.

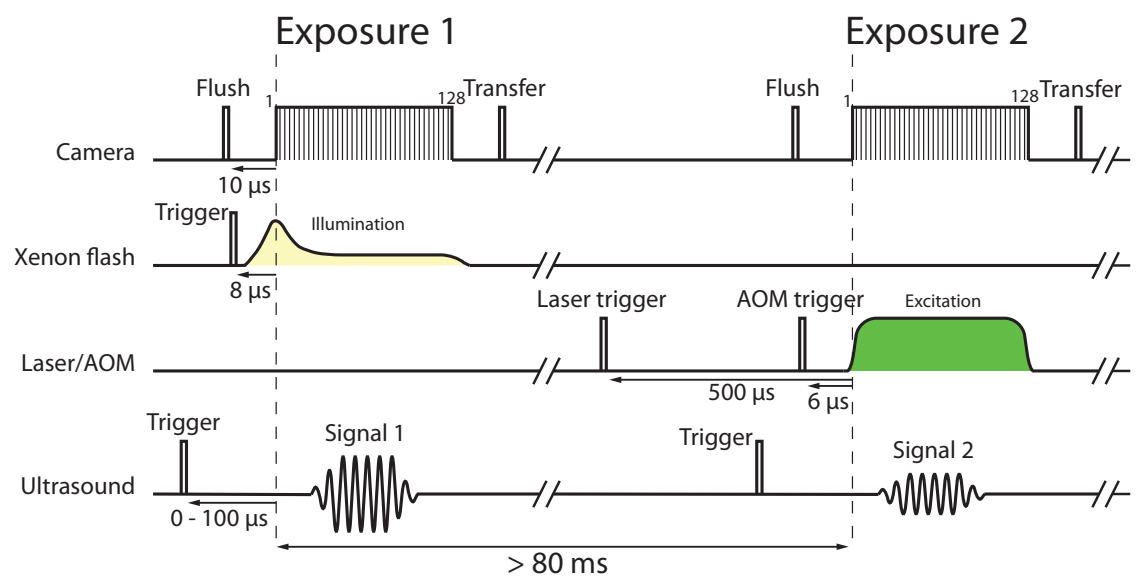

Figure 2.10: Timing diagram for two subsequent recordings within a typical experiment. Before each exposure the CCD's are flushed and after capturing frame 128 the image data is transferred to the internal RAM. The xenon flash is triggered several microseconds before exposure 1 to start a bright field recording and before exposure 2 the laser and the AOM are triggered to initiate the fluorescence recording. Separately pre-programmed ultrasound signals are sent for each exposure. 


\subsection{RESULTS}

\subsection{Results}

\subsubsection{Microbubble spectroscopy using ROI mode}

In previous research the segmented mode was used to perform a large set of experiments in a single run of the Brandaris 128. Van der Meer et al. subjected a single microbubble to a scan of 24 different frequencies to construct a resonance curve by using 4 segments of 32 frames whereas Emmer et al. [20] used 12 segments of 64 frames to vary the pressure for a constant frequency. Overvelde et al. [3I] operated the Brandaris 128 in the normal mode to obtain the behavior of a single microbubble for 8 different pressures and 12 different frequencies. In order to use all 128 frames for each exposure it required in total 16 experiments which takes in total approximately half an hour to record.

By using the ROI mode, Faez et al. [32] were able to record 49 exposures of a single microbubble within a single run of the camera, with the purpose to study its subharmonic behavior. In the first exposure no ultrasound was applied to determine the resting radius of the bubble. In the subsequent 48 exposures the frequency was swept in 16 steps from 4 to $7 \mathrm{MHz}$ at an acoustic pressure of $50 \mathrm{kPa}$, followed by 16 frequency steps at $100 \mathrm{kPa}$ and finally 16 frequency steps at $120 \mathrm{kPa}$. Thus within 5 seconds 3 resonance curves of a single microbubble were measured. From each exposure the diameter of the bubble as a function of time was determined. From this the maximum amplitude of oscillation at the subharmonic frequency was extracted and plotted against the frequency as shown in figure 2.17 .

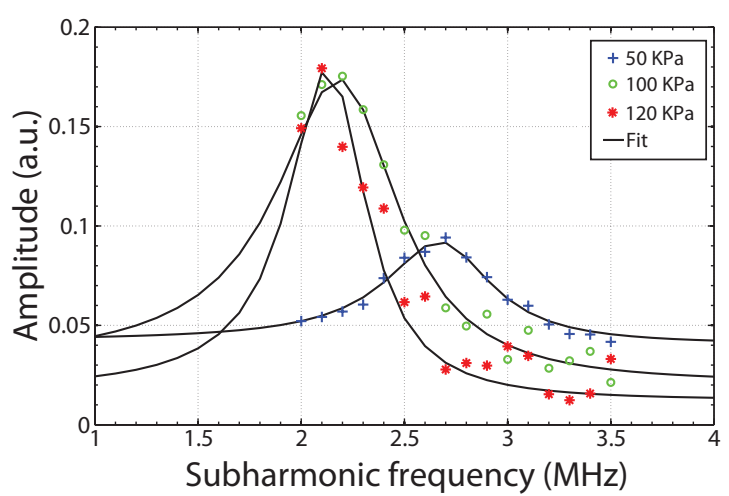

Figure 2.11: Three resonance curves of the subharmonic response of a single microbubble. All curves were measured within 5 seconds in a single run of the camera. 


\subsubsection{Imaging at multiple time scales using segmented mode}

A typical recording of the Brandaris 128 at $10 \mathrm{Mfps}$ has a duration of $13 \mu \mathrm{s}$. This is very short compared to the time between recordings, which is $80 \mathrm{~ms}$ or longer. Two consecutive recordings can therefore be considered to be completely separate experiments, as the objects in the field of view have sufficient time to return to an equilibrium state after ultrasonic excitation. By decreasing the inter-segment time down to the order of microseconds, transient effects can be studied in more detail, whilst maintaining the high time resolution within the recording itself. It is known, for instance, that lipid shedding of phospholipidcoated microbubbles due to large amplitude oscillations causes shrinkage of a bubble over time [33]. The improved segmented mode allows us to study this effect at much shorter timescales.

As an example of the improved segmented mode the frame rate was set to $10 \mathrm{Mfps}$, resulting in a mirror period of $120 \mu \mathrm{s}$. The mirror mounted during the experiment had three reflective sides, which allowed for an inter-segment time of $40 \mu \mathrm{s}$ or any multiple of that number. In this case the inter-segment time was set to $80 \mu \mathrm{s}$.

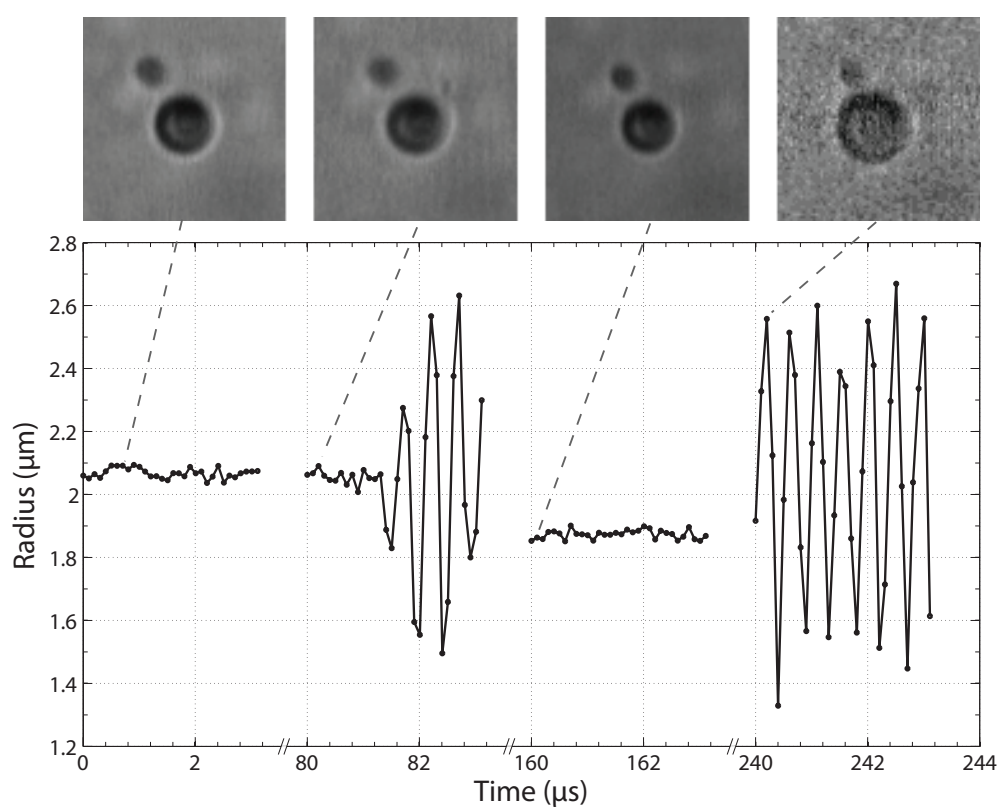

Figure 2.12: The radial excursion of a microbubble in four segments with an intersegment time of $80 \mathrm{~ms}$. The bubble was insonified at $2.25 \mathrm{MHz}$ in segment 2 and 4 . The difference in resting radius between segments 2 and 3 indicates bubble shrinkage on a microseconds timescale. 


\subsection{RESULTS}

The 128 CCD's were divided into four segments of 32 frames. A phospholipidcoated microbubble was insonified at $2.25 \mathrm{MHz}$ in segments 2 and 4. Figure $\mathbb{2 . 1 2}$ shows the radial excursion of the microbubble in all segments, where oscillations are visible in the second and fourth segment. The trigger of the ultrasound signal was not corrected for the added delay of 64 frames between segment two and four, causing the bubble to start to oscillate before the start of the last segment. Furthermore, the pulse length of the xenon flash was increased to fully cover all segments, however, a decrease in the light intensity in the final segment was still observed. The decrease in the resting radius of the bubble between segments 2 and 3 shows that bubble shrinkage can be significant on a timescale of $80 \mu \mathrm{s}$.

\subsubsection{Ultra high-speed fluorescence imaging of microbubbles}

Phospholipid-coated microbubbles, to be used as ultrasound contrast agents, were produced in-house following the method of Klibanov et al. [34] In the final production step the fluorescent dye Dil was mixed together with the microbubble solution to incorporate it into the phospholipid shell. The fluorescent microbubbles were washed to remove excess dye. The final concentration of dye molecules in the lipid shell was low, considering that Dil is located in between the DSPEPEG molecules that make up only $5 \%$ of the total shell material.

The microbubbles were put in an acoustically and optically transparent OptiCell $^{\mathrm{TM}}$ container and insonified at a frequency of $300 \mathrm{kHz}$ with a pressure amplitude of $150 \mathrm{kPa}$ for a period of 10 cycles. A single microbubble was visualized using a $60 \times$ water-immersion objective. The microbubble oscillations were recorded alternatingly in bright field (exposure 1,3 , and 5) and in fluorescence (exposure 2, 4, and 6) at a frame rate of $1.5 \mathrm{Mfps}$

Figure 2.13 a and $b$ show still frames from the bright field and the consecutive fluorescence exposure. The radial intensity profiles, depicted in figure $[2.13]$, show the complex ring structure of the bright field image, due to diffraction and Mie scattering from the microbubble of the bright field illumination. The fluorescence intensity profile shows a much simpler profile of the microbubble with a much higher contrast; the contrast ratio between the center of the bubble and the background is 1.5 for the bright field image and 13 for the fluorescence image. Furthermore, while the bright field images miss all information of the nanometer coating material, the fluorescence images show inhomogeneities in the distribution of dye molecules over the bubble shell.

The microbubble oscillations were analyzed using Matlab. The contour of the bubble was tracked in each frame by finding the maximum/minimum slope for all angular positions on the bubble rim. The resulting radius-time curve is 

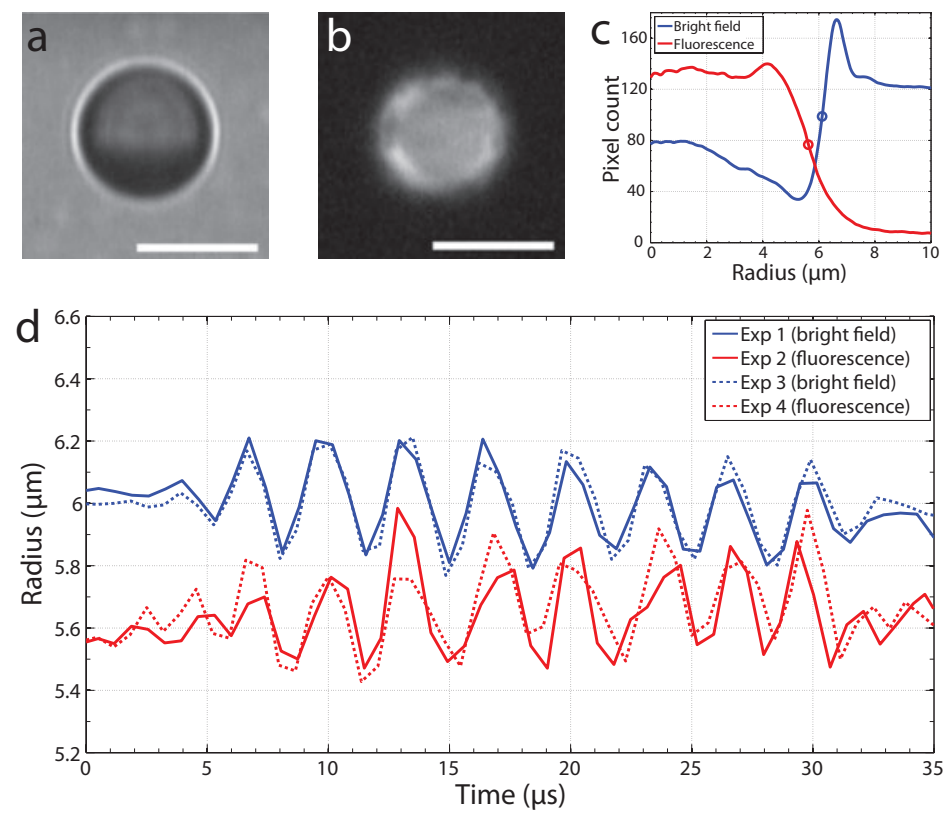

Figure 2.13: a) Still frame from the bright field exposure and b) from the fluorescence recording of an ultrasound contrast agent microbubble. Scale bars indicate $6 \mu \mathrm{m}$. c) Radial intensity profiles for bright field imaging (blue) and fluorescence imaging (red). The circles indicate the maximum of the first derivative of the profile, where the radius is determined by the analysis. d) Radius time curves of the microbubble oscillations. The bright field recordings (blue) show a larger radius than the fluorescence recordings (red).

plotted in figure $2.13 \mathrm{~d}$. The bright field traces show great similarity as do the fluorescence traces. Both imaging methods capture the dynamics of the bubble oscillations, however, there is a systematic error in the measured radius for the two methods. This can also be seen in figure $2131 c$, where the minimum slope of the fluorescence image is located at a smaller radius than the maximum slope of the bright field profile.

This example demonstrates the importance of fluorescence imaging at these length scales, where diffraction and Mie scattering dominate the bright field image of the bubble with a size in the order of the wavelength of the light. Besides the optical imaging system, i.e. the objective lens, mirror, relay lenses etc., the illumination system also influences the final image. Because the fluorescence light originates from the object itself, the final image does not suffer from complex scattering and diffraction. This problem is addressed in more detail in chapter 3. 


\subsection{DISCUSSION}

In order to generate sufficient fluorescence signal the excitation irradiance levels have to be substantial, causing photobleaching of the excited dye molecules, even at the microsecond time scale. Figure $[2.14$ shows the relative mean fluorescence intensity of the microbubble depicted in figure [2.13] over the course of a single exposure. Within $5 \mu \mathrm{s}$, the intensity has dropped to half of its original value, resulting in a significant loss of signal-to-noise ratio. Experiments with bubbles that were not excited by ultrasound showed that the photobleaching does not influence the measured radius (see section 3.2). The second curve indicates that the photobleaching is largely reversible indicated by an initial intensity of $84 \%$ compared to the first fluorescence exposure. The delay between the two exposures was $500 \mathrm{~ms}$.

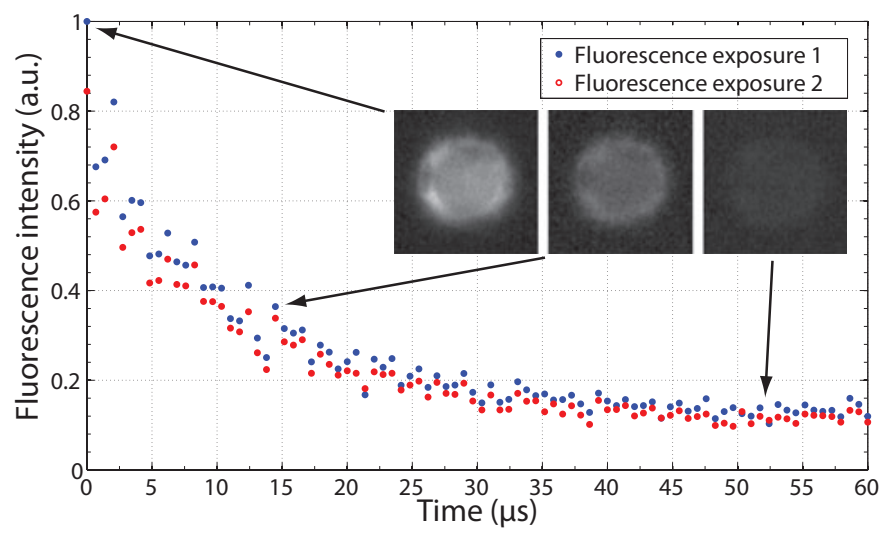

Figure 2.14: Fluorescence intensity of a fluorescently labeled microbubble as a function of time. The blue circles show the relative fluorescence intensity over the period of the first exposure to continuous laser excitation light. In the second exposure (red circles) the fluorescence partially recovers to $84 \%$ of the initial value.

\subsection{Discussion}

The region of interest mode has greatly enhanced the capabilities of the Brandaris 128 , by allowing a single experiment to capture the behavior of a microbubble for a large parameter set. It drastically reduces the record time, it limits the wear of the turbine and it saves on the usage of helium. Furthermore, a microbubble can be studied extensively in a short period of time, reducing the influence of experimental parameters such as temperature, shell composition, local gas concentration etc. However, the increased number of recordings per experiment causes a longer download time to transfer the image data to the PC, as the images are made to fit the full-frame image size by adding a black 
background around the ROI. Nevertheless, a significant time gain is achieved.

Using the segmented mode, multiple timescales can now be captured within a single recording in order to study phenomena with high temporal resolution over a prolonged period of time. The challenge in this operating mode is the bright field illumination, since only a single discharge from a xenon flash light source can be used and high input energy levels are required that lead to increased deterioration of the lamp. Employing multiple light sources coupled into a single optical fiber can be used to overcome this problem, although this would still limit the number of segments. Continuous light sources are not an option, as they do not reach the required light intensity, and laser bright field illumination leads to severe loss in image quality due to speckles and interference patterns, caused by the spatial and temporal coherence of the light.

To our knowledge this is the first time fluorescence recordings of an oscillating microbubble at a frame rate exceeding $1 \mathrm{Mfps}$ and a submicron resolution have been performed. This demonstrates that the sensitivity of the Brandaris 128 imaging facility has been increased tremendously. This opens up an exciting new field of research applications for the camera, allowing for the visualization of phenomena such as ultrasound-triggered drug release and uptake, and flow visualization using nanoseconds time-resolved $\mu$ PIV and PTV. Since no transmitted light is required for fluorescence imaging and the bubble-background contrast is greatly enhanced, optical in vivo studies of ultrasound contrast agents can be performed. In all experiments, but especially when biological samples are used, thermal effects of the laser illumination due to unwanted absorption have to be considered. When long pulses are used for imaging at a lower frame rate or when the concentration of dye is very high, care should be taken to avoid heating and possibly vaporization. Furthermore, the amount of photobleaching has to be minimized, to ensure a sufficient signal-to-noise ratio. Since the photobleaching depends on many parameters, such as pulse length, laser intensity, spot size, but also dye concentration, an assessment should be made for each individual experiment to find a compromise between fluorescence yield and photobleaching.

With all these new features in place, one issue remains unresolved, namely the maximum frame rate of the camera. Redesigning the optical configuration by placing the image arc further away from the mirror to fit more channels in is an option, although the costs would be very high and it would result in a reduced numerical aperture. Driving the turbine at higher speeds requires minimizing the viscous drag by running it on hydrogen instead of helium or by running it in a vacuum. Even if this can be achieved, the stability of the turbine system can then become the limiting factor. A increase in speed of a factor of two can theoretically be achieved by splitting the beam coming from the microscope 


\subsection{CONCLUSIONS}

and thereby making two separate images per CCD-sensor. However, the NA of the camera would become a factor 2 lower in addition to the reduced exposure time, resulting in a real challenge to collect sufficient photons per image.

\subsection{Conclusions}

At its introduction ten years ago, the Brandaris 128 was already a unique ultrahigh-speed imaging facility. The improvements that have been made in the last decade further contribute to the versatility of the system. By increasing the number of frames from 768 to 16000 , a single run of the camera can record up to 125 unique recordings within several seconds. The time between separate recordings has been reduced by three orders of magnitude from $80 \mathrm{~ms}$ to $17 \mu \mathrm{s}$, enabling imaging at multiple timescales ranging from nanoseconds to milliseconds. The incorporated fluorescence module unites all the advantages of fluorescence imaging such as enhanced contrast and resolution to time-resolved ultra-high-speed imaging. Combined with the maximum frame rate of $25 \mathrm{Mfps}$, the Brandaris 128 remains an exceptional camera with great potential for future research. 


\section{3}

\section{Optical sizing of ultrasound contrast agent microbubbles: bright field versus fluorescence microscopy}

Accurate sizing of single ultrasound contrast agent microbubbles is crucial to model their behavior as a function of the applied ultrasound field. Bright field microscopy is used to obtain the bubble radius, however, transmitted light microscopy results in a complex bubble image, due to diffraction and scattering of the illumination light interacting with the bubble. It is difficult to model the resulting radial intensity profile, rendering bright field microscopy unsuitable for the sizing of (sub)micron-sized bubbles. Fluorescence microscopy of fluorescently labeled microbubbles results in a much simpler bubble image, as the collected light does not interact with the bubble itself. Furthermore, the out-of-focus error for this technique is smaller. The comparison of both techniques has lead to an empirical relation between the bubble radius obtained in bright field and in fluorescence microscopy. This result has been applied to successfully correct the data from a recent bubble dynamics study. 


\subsection{INTRODUCTION}

\subsection{Introduction}

Microbubbles have been used as ultrasound contrast agents (UCAs) for over thirty years. These coated gas-filled agents with a diameter between 1 to $10 \mu \mathrm{m}$ have been studied extensively to optimize their acoustic response to the applied sound field. Microbubbles display a strong resonance behavior at frequencies used for medical ultrasound imaging, which makes them very efficient scatterers for organ perfusion imaging. In the linear approximation the resonance of a gas bubble can be described by two parameters: its frequency and size. To fully capture the dynamics of a coated microbubble and predict the scattered sound field from the applied sound field, complex physical models have been devised based on the Rayleigh-Plesset equation. The applied acoustic pressure and frequency can be controlled accurately. However, the time-evolution of the bubble radius depends nonlinearly on the applied acoustic pressure and the scattered signal depends nonlinearly on the radial dynamics of the bubble. Besides the first and second derivative of the radius as a function of time, the bubble radius itself is also a parameter in the equation. Therefore knowledge of the absolute bubble radius is elementary to validate bubble dynamics models and to characterize viscoelastic shell parameters.

In the last decade, optical imaging of a bubble's behavior has become more important, due to the introduction of digital cameras capable of ultra-highspeed imaging ( $>1 \mathrm{Mfps}$ ). In addition to acoustic measurements it provides time-resolved information of the bubble size and shape. This has led to the discovery of several new phenomena, such as "compression-only" behavior [2], shell buckling [19], and shape oscillations [3]. In other fields of research, highspeed optical imaging has also become an valuable tool to accurately determine the position and size of microscopic objects, such as droplets in piezo inkjet printing [35], microjets generated by vapor bubbles [36] and microbubbles for semiconductor surface cleaning [26].

Various other methods have been used to size ultrasound contrast agent microbubbles. Acoustic sizing is a method to measure the size of a microbubble in vivo and noninvasively [37], however, it relies on optical reference measurements. Large bubble populations can be analyzed using a Coulter Counter [38] based on the electrozone sensing method, providing only statistical information about the bubble size [39]. The size distribution can also be measured using dynamical light scattering and in addition, this technique can be applied to measure the time-resolved radius of a single microbubble in an acoustic setup, as demonstrated by Guan and Matula [40]. A high temporal resolution can be obtained for a prolonged period of time compared to high-speed imaging. However, the measured scatter is determined by all scattering particles within the 
laser spot, which requires single microbubbles in a large volume to avoid bias signals from nearby objects. Furthermore, no information about the shape of the bubble is obtained. Electron microscopy can resolve nanometer sized structures on the shell of a UCA in both scanning electron microscopy (SEM) [41] and transmission electron microscopy (TEM) [23, 42], however, preparation of the microbubble sample is required since gas-filled objects are not compatible with the low-pressure environment. Also, SEM and TEM cannot be performed in real-time during the ultrasound experiments. The same argument holds for atomic force microscopy (AFM), another technique that offers a high spatial resolution, which has been used by Sboros et al. [43] to assess the mechanical properties of a microbubble.

Due to their size, optical microscopy is required to visualize microbubbles and transmitted light is the common illumination technique. Especially when timeresolved recordings at high frame rates are required, this illumination method is chosen because of its yield in terms of light intensity levels. A drawback of this method is that it leads to a complex bubble image, as shown in figure $3.1 \mathrm{~b}$. The image does not just depend on the imaging system, i.e. the microscope objective and tube lens, also the illumination plays an important role since refraction, diffraction and scattering alter the pathways of the light rays incident on the bubble surface. After the light has passed through all the optical elements

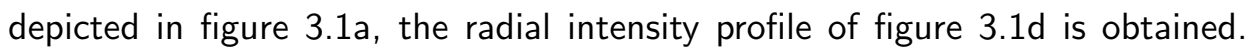
It is generally assumed that the actual bubble radius lies on the steepest slope of the profile, going from the wide dark ring to the thin light ring. There are multiple ways to determine the radius, either by using a threshold gray level value or by using the point with the highest derivative [18, 44, 45]. No method is supported by theory or calibrated experimentally, by comparing results of a different technique with a much higher resolution limit than the wavelength of light, such as SEM, TEM or AFM.

Because the size of a microbubble is of the same order as the wavelength of light, geometrical optics or ray tracing cannot be used and Mie theory [46] has to be applied to calculate the interaction between the incident light and the microbubble. This produces a complex angular intensity profile to be emitted from the position of the bubble, causing a ring-shaped diffraction pattern in the far field and the central spot in the bubble image. In addition to the light emitted by the bubble the unperturbed light surrounding the bubble has to be taken into account as well. As Mie scattering accounts for the light passing through the bubble, the bubble can then be regarded as a circular obstruction and light rays passing this obstruction will interfere behind the bubble and cause an added diffraction pattern both in the near field and far field. The near field (Fresnel) diffraction will be imaged by the objective and cause the 


\subsection{INTRODUCTION}

a

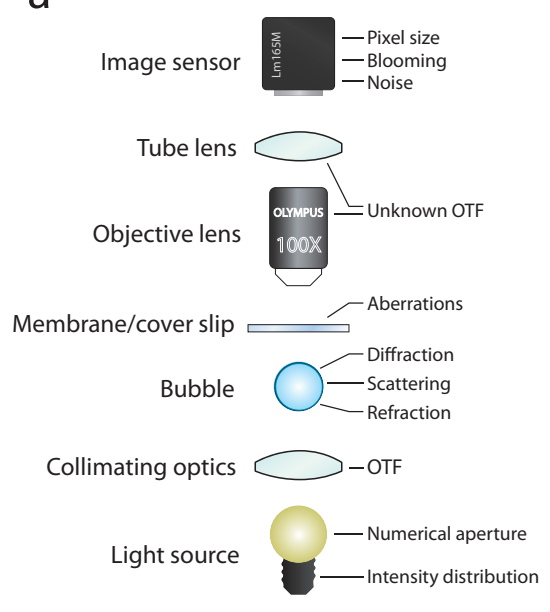

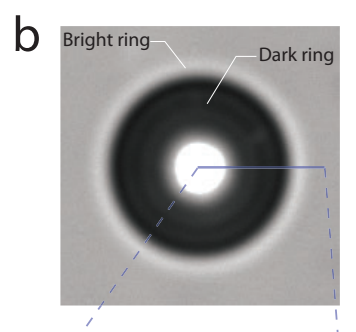

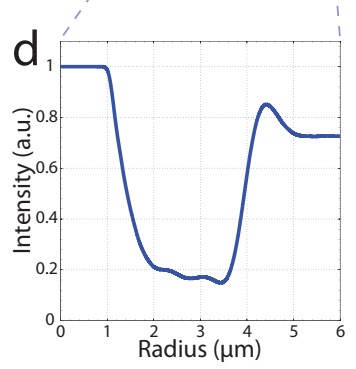

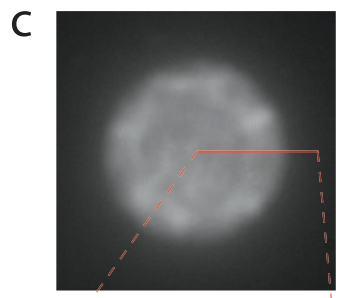

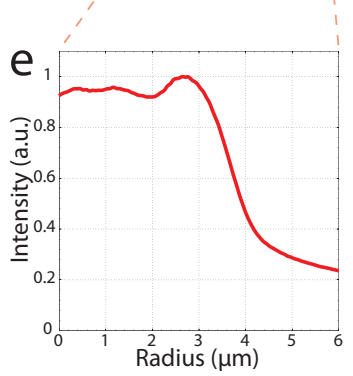

Figure 3.1: a) Schematic representation of the optical system used in transmitted light microscopy. b) A single, coated microbubble with a radius of approximately $4 \mu \mathrm{m}$ in bright field and fluorescence $(c)$, and their corresponding averaged radial intensity profiles in bright field (d) and fluorescence (e).

dark and bright rings in the image. The optical transfer function (OTF) of the microscope system itself will add abberations to the image and the OTF acts as a low-pass filter, since the resolution of the system is limited by the NA of the microscope objective. This will reduce the slope of the transition from the obstruction to the background. For commercially available objectives, consisting of numerous lenses, the OTF is unknown. Consequently obtaining a full mathematical solution of the image formation of a microbubble illuminated by transmitted light can be considered to be challenging and is beyond the scope of this chapter.

By adding a fluorescent dye to the shell material of a microbubble, fluorescence microscopy can be used to optically record a bubble's behavior. Because the low photon yield of this method compared to bright field microscopy, it is not a common imaging technique used in time-resolved ultra-high-speed recordings. However, fluorescence imaging does not suffer from a complex relation between the pathways of the excitation light and the emission light, since the direction of the fluorescence photons is random. Part of the emission light, coming from the bottom of the microbubble, will interact with the bubble interface before reaching the objective, but the majority of emitted photons will be captured by 
the objective without passing any water-air interface. The resulting image is therefore much simpler, as can be seen in figure [3.]c. The corresponding radial intensity profile in figure 3 . Ile shows a step-like function going from the interior of the bubble to the background area. A rim is visible, caused by the fact that the signal coming from the part of the bubble within the depth of field of the objective gives the highest signal. It is assumed that the bubble radius lies on the transition from this bright rim to the dark background. The width of this transition is largely determined by the resolution of the imaging system.

The resolution of fluorescence microscopy is still limited by the wavelength of light and the NA of the objective, however, we believe that it offers a reference to be used as a calibration method for bright field recordings of microbubbles. Therefore we present the results of a comparison between bright field microscopy and fluorescence microscopy for optical sizing of microbubbles. For this purpose a population of fluorescently-labeled, phospholipid-coated microbubbles was imaged in bright field and in fluorescence to quantify the difference in measured radius between the two methods. Furthermore, several error sources and their influence on the measured radius are discussed. Finally, the outcomes are used to correct the results from a recently published paper on the predicted acoustic signal of a single microbubble based on the optically measured radius-time curve.

\subsection{Materials and methods}

\subsubsection{Microbubbles}

Phospholipid-coated microbubbles with a $\mathrm{C}_{4} \mathrm{~F}_{10}$ gas core were produced inhouse following a method described by Klibanov et al. [34] The lipophilic dye DilC18 was incorporated into the monolayer shell, located in between the DSPEPEG molecules that made up approximately $5 \%$ of the total shell material. The location of the dye ensured that the difference in radius for a labeled or unlabeled microbubble was in the order of several nanometers, well below the resolution limit of our imaging system. The microbubbles were dispersed in water and injected into a channel of an microslide ( $\mu$-slide, iBidi, Germany). The slide was put under the microscope as depicted in figure 3.2. The concentration of bubbles was such that multiple bubbles were visible in the field of view of the camera and that the distance to neighboring bubbles would be large enough to avoid interference during the measurement. A total of 218 bubbles were measured and analyzed. 


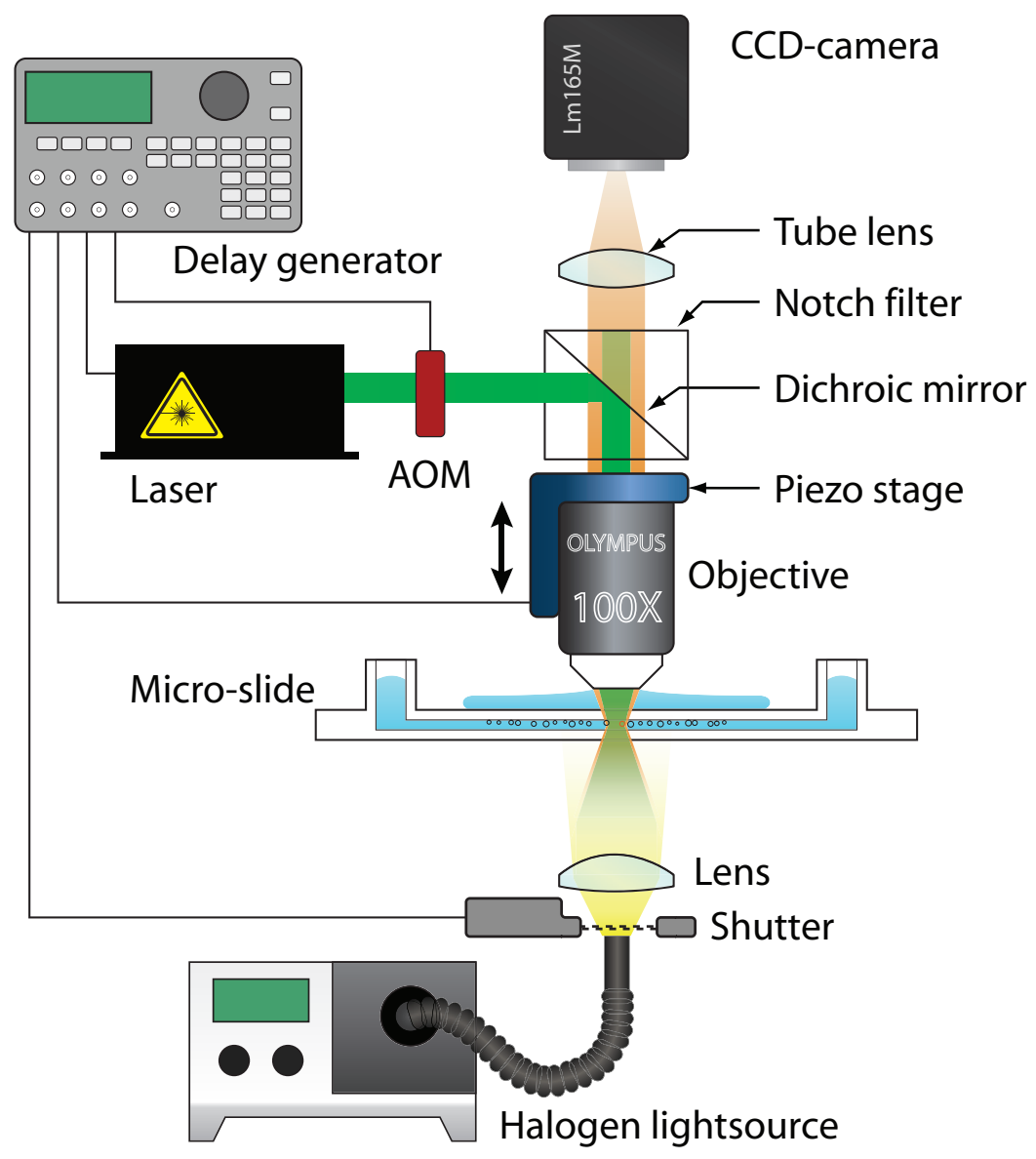

Figure 3.2: Schematic representation of the experimental setup.

\subsubsection{Experimental setup}

The experimental setup consisted of an upright microscope (BX-FM, Olympus, USA) fitted with a $100 \times$ water-immersion objective with a NA of 1.00 , mounted onto a vertical piezo translation stage (Jena MIPOS 250, Jenoptik, Germany) with a resolution of $5 \mathrm{~nm}$, operating in closed loop. On top of the microscope a monochrome CCD-camera ( $L m 165 \mathrm{M}$, Lumenera, Canada) was mounted with a pixel size of $6.45 \mu \mathrm{m}$.

In order to avoid motion blur due to Brownian motion, the bright field and fluorescence illumination were pulsed with a maximum pulse duration of $10 \mathrm{~ms}$. The diffusion length of a microbubble on this timescale is on the order of several 
nanometers, as can be approximated using the Einstein-Stokes equation. To achieve sufficient fluorescence excitation intensities at such short pulse durations, whilst maintaining a homogeneous light distribution over the complete field of view, a continuous wave (CW) DPSS laser (Cohlibri, Lightline, Germany) with a wavelength of $532 \mathrm{~nm}$ was employed. Coupled to an acousto-optic modulator (AOM) (AOTF.nC-VIS, AA Optoelectronic, France) high intensity laser pulses with a duration of less than a millisecond were produced. Bright field illumination was supplied by an $8 \mathrm{~mm}$ glass fibre, coupled to a $150 \mathrm{~W}$ halogen light source (KL1500LCD, Schott, Germany). An electronically controlled shutter (SH05, Thorlabs, Germany) enabled flash illumination with a minimum flash duration of $10 \mathrm{~ms}$. The light emitted by the optical fibre was collimated by a spherical lens. The timing of the experiment was controlled by a digital delay generator (Model 575, Berkeley Nucleonics, USA) that was programmed to trigger the shutter for a bright field image, the AOM for a fluorescence image and the piezo stage to displace the objective in the vertical direction.

\subsubsection{Measurement protocol}

First, a suitable measurement region in the channel was located with multiple bubbles at sufficient distance from each other and with comparable radius. Secondly, the focal plane was determined manually by eye. To start the experiment the piezo stage was lowered by $5 \mu \mathrm{m}$ and for 21 vertical positions, with a step size of $500 \mathrm{~nm}$, a bright field image and a fluorescence image were recorded. A selection of the resulting image sequence is depicted in figure 3.3. As the microscope objective scans through the bubble, starting with the bubble in between the focal plane and the objective (proximal-to-focus), the blurred bright field and fluorescence images come into focus. When the bubble is distal-to-focus the bright field images display a distinct ring pattern, whereas the fluorescence images even show the distribution of dye on the bubble top surface.

The step size of $500 \mathrm{~nm}$ was chosen, based on the depth of field (DOF) of the microscope system. The DOF can be calculated by the following equation [47]:

$$
\mathrm{DOF}=\frac{\lambda n}{\mathrm{NA}^{2}}+\frac{n}{M \mathrm{NA}} e,
$$

where $\lambda$ is the wavelength of the light, $n$ is the refractive index of the immersion liquid, $M$ is the magnification of the objective, NA is the numerical aperture and $e$ is the smallest distance resolved in the image plane, i.e. the pixel size of the CCD sensor. For our system this gave a DOF of approximately $1 \mu \mathrm{m}$. Using a step size of $500 \mathrm{~nm}$ ensured that for each bubble at least one image was taken that included the plane where the bubble was in focus, at the vertical position where the width of the bubble is maximum. 


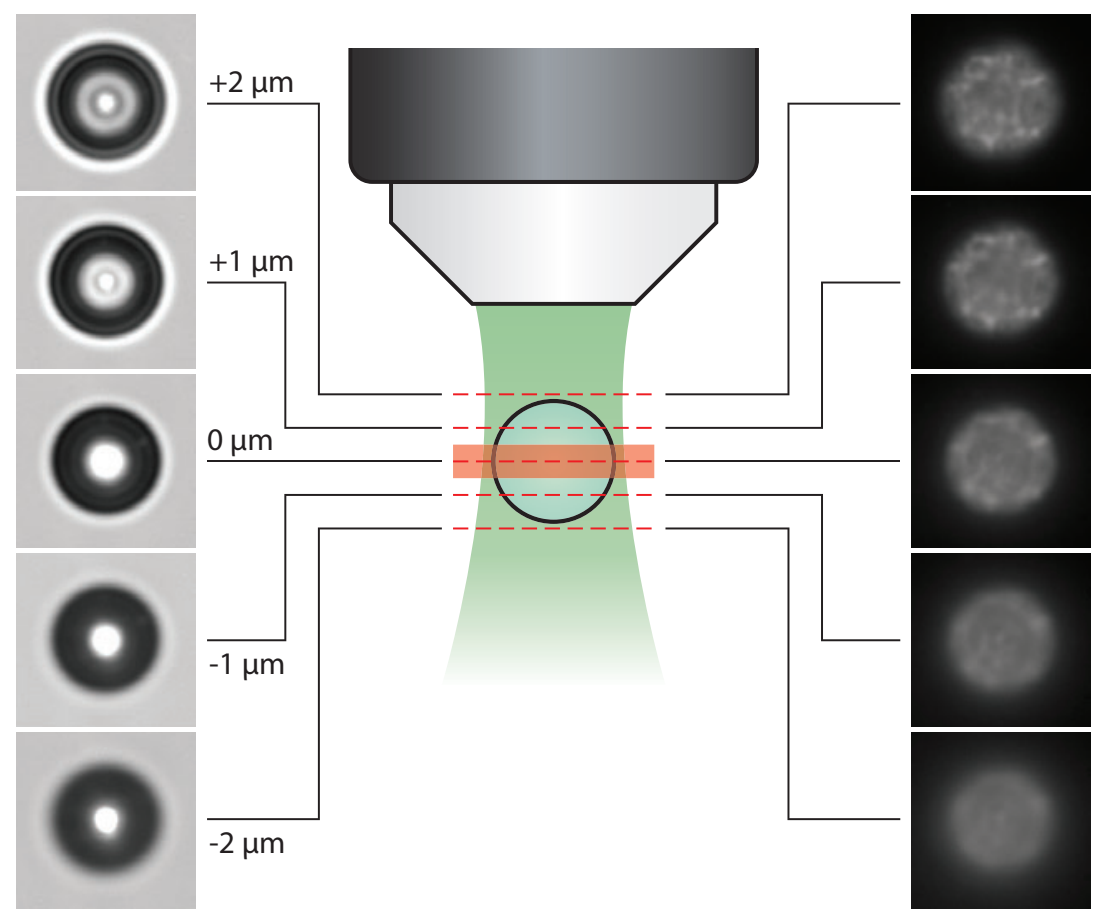

Figure 3.3: Overview of a single measurement. On the right several bright field frames are depicted, recorded at the indicated vertical positions. On the left are the corresponding fluorescence images. The red marked area indicates the size of the depth of focus.

\subsubsection{Image analysis}

Each image sequence was segmented to obtain a vertical stack of bright field and fluorescence images for every microbubble. For each image in a stack the center position of the bubble was determined. The image was then transformed into polar coordinates around this point. Each line of the transformed image contains a radial intensity profile. The average profile over all angles was taken and the derivative of this line with respect to the radius was determined. The location of the maximum value of the derivative (or minimum for a fluorescence image) was measured to obtain the radius for each bubble image.

To determine the correct vertical position of the focal plane the derivatives of the fluorescence images were compared to find the intensity profile with the steepest transition from bubble to background [48]. The corresponding image was assumed to be at the focal plane of the microscope objective, allowing for the allocation of a vertical position relative to this plane for all other images of the corresponding bubble. 


\subsubsection{Error sources}

\section{Bright field: illumination}

In a transmitted light microscope a condenser is used to focus the light onto the sample and to match the incoming rays of light to the NA of the objective lens. In principle two illumination techniques can be used to obtain the maximum resolution: Köhler illumination or critical illumination [49]. The first produces a very homogeneous illumination pattern as the image of the light source is fully defocused at the specimen. However, the light intensity is not as high as for critical illumination. On the other hand, using the second technique an image of the light source filament is formed at the specimen plane, causing an uneven background. In the case of Köhler illumination, the wavefront of light reaching the object plane has a high spatial coherence compared to critical illumination. This produces unwanted diffraction fringes in the image due to increased interference of the coherent wavefronts.

In a typical ultrasound setup, where the sample is placed in a water bath, Köhler illumination or critical illumination often cannot be applied, because the necessary optics would interfere with the acoustical pathways. Therefore optical fibers are used to direct the light from the source to the object plane. By using an optical fiber, the primary light source is replaced by the fiber end which acts as a secondary light source with a different spatial distribution, whilst maintaining the same opening angle as the light coupled into the fiber. In our case a glass fiber was used consisting of 400 small fibers with a total circular aperture of $8 \mathrm{~mm}$ in diameter. For many applications, the light emitted from the fiber end has sufficient intensity, however, the NA of the light source is reduced significantly when the distance from the fiber end to the object plane is increased. By using a simple collimation lens close to the fiber end the light can be focused onto the object, increasing the NA and the light intensity, without obstructing the acoustic signals.

The influence of the NA on the radial intensity profile was investigated by comparing the bubble image for three illumination options: (1) no collimation lens, which resulted in a NA of $0.1,(2)$ approximate Köhler illumination, by putting the fiber end at the back focal point of the lens, resulting in a NA of 0.25 and (3) approximate critical illumination, where an image of the fiber end was made just above the object plane to avoid an inhomogeneous illumination, resulting in a NA of 0.5 . The resulting images and their radial intensity profiles are depicted in figure 3.4 .

The intensity profile obtained with the unfocused light displays the most pronounced diffraction rings, which become less clear when the NA is increased. This indicates that the light from the fiber has the highest temporal coherence. 


\subsection{MATERIALS AND METHODS}

a

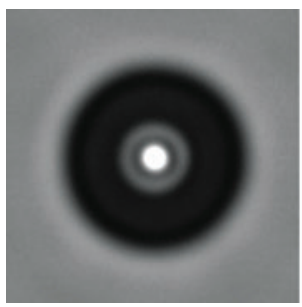

$\mathrm{NA}=0.1$

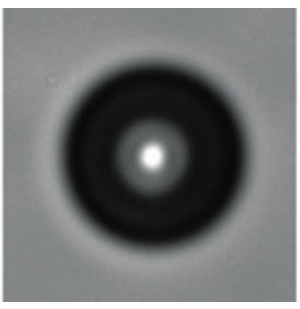

$\mathrm{NA}=0.25$

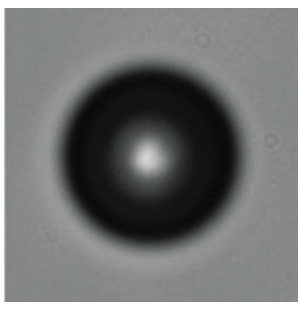

$\mathrm{NA}=0.5$

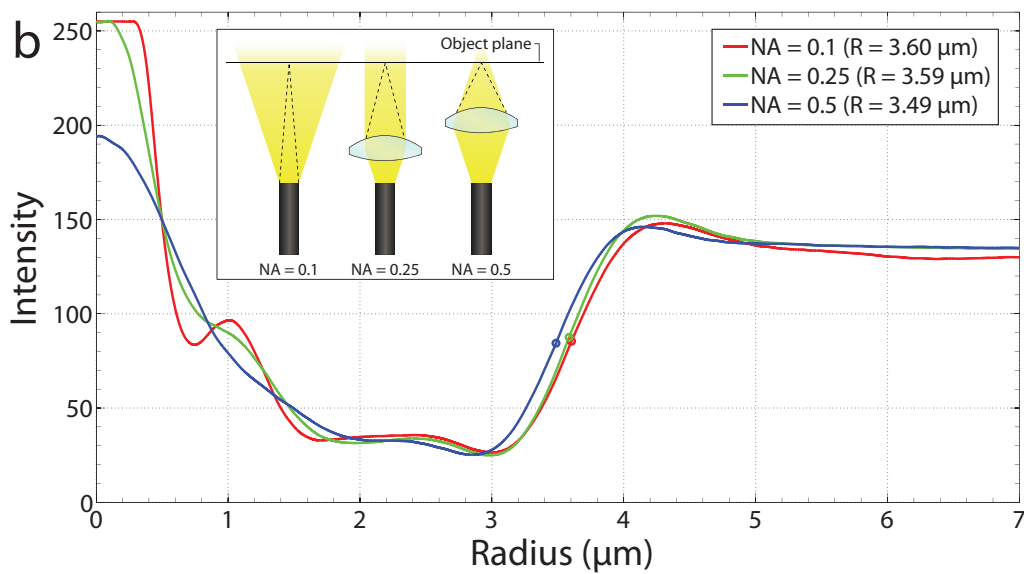

Figure 3.4: Influence of NA of the light source on the bubble intensity profile. a) Bright field images of a single bubble illuminated using different NA. b) Corresponding radial intensity profiles.

The determined radius is marked by the circles in figure $3.4 \mathrm{~b}$, which show a small decrease in radius with increasing NA. Interestingly, at the highest NA, the optical contrast of the bubble is the lowest. This could be attributed to the refraction of photons that impinge on the bubble under a large angle with respect to the optical axis. When they are refracted, this angle will become smaller and they fall within the limits of the NA of the microscope objective.

Since the light intensity may vary from experiment to experiment or even from image to image in a single recording, the influence of the background light intensity on the measured radius was determined. The intensity profile of a bubble for 9 different light intensities was measured and is plotted in figure 3.5. When the intensity profiles are normalized to the background intensity, depicted in the inset of figure [.5.5, the curves collapse into each other. This is also reflected in the measured radii: the variation is less than $1 \%$. 


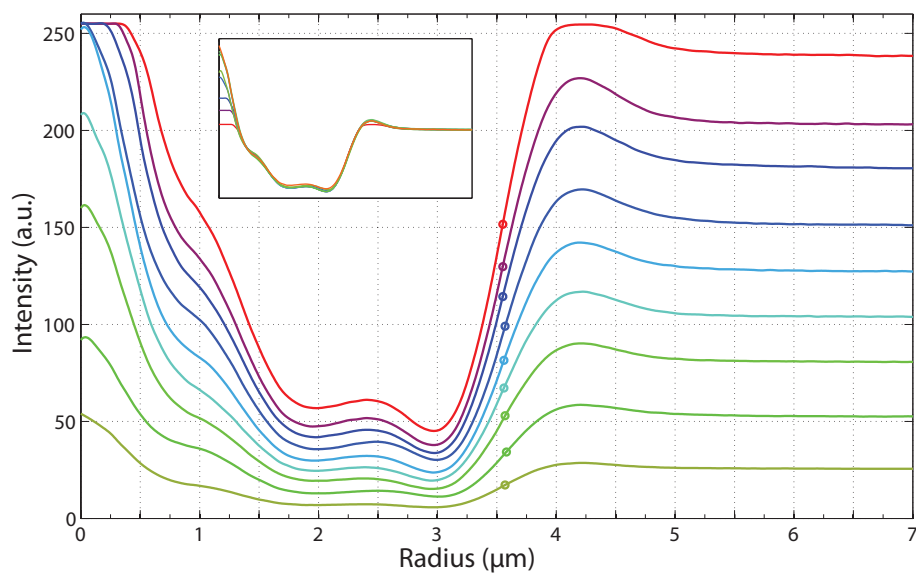

Figure 3.5: Influence of transmitted light intensity on the bubble intensity profile. The normalized curves (inset) collapse into each other.

\section{Fluorescence: photobleaching}

In fluorescence imaging the fluorescence emission decreases over time due to photobleaching. When a high temporal resolution is required, the excitation light intensity must be high, given the short exposure times of a millisecond or shorter, and significant bleaching can occur. To evaluate whether the measured radius would change when the fluorescence is bleached, a single microbubble was brought into the focal plane of the microscope and illuminated at a high laser intensity for a number of pulses. For each laser pulse the fluorescence was imaged and a selection of the resulting intensity profiles is shown in figure $3 . \mathrm{B}$ a and $b$ together with the recorded frames. The inset shows the normalized intensity profiles.

As the intensity decreases after each pulse, the measured radius increases up to several percent. The normalized intensity profiles show that the pronounced rim decreases rapidly with the number of applied laser pulses. This causes the point of the highest derivative to shift outwards. The deviation at lower fluorescence intensities increases as well. This can be attributed to the decrease in the steepness of the transition from background to bubble, which causes a larger error in the determination of the point of the highest derivative. In this bleaching experiment the error in the measured radius was below $1 \%$ up to 15 laser pulses. This corresponds to a maximum fluorescence intensity of 120 on a gray-scale level. During the sizing experiments, the laser intensity was adjusted to minimize photobleaching and resulting fluorescence intensities were above 120. 


\subsection{MATERIALS AND METHODS}

a
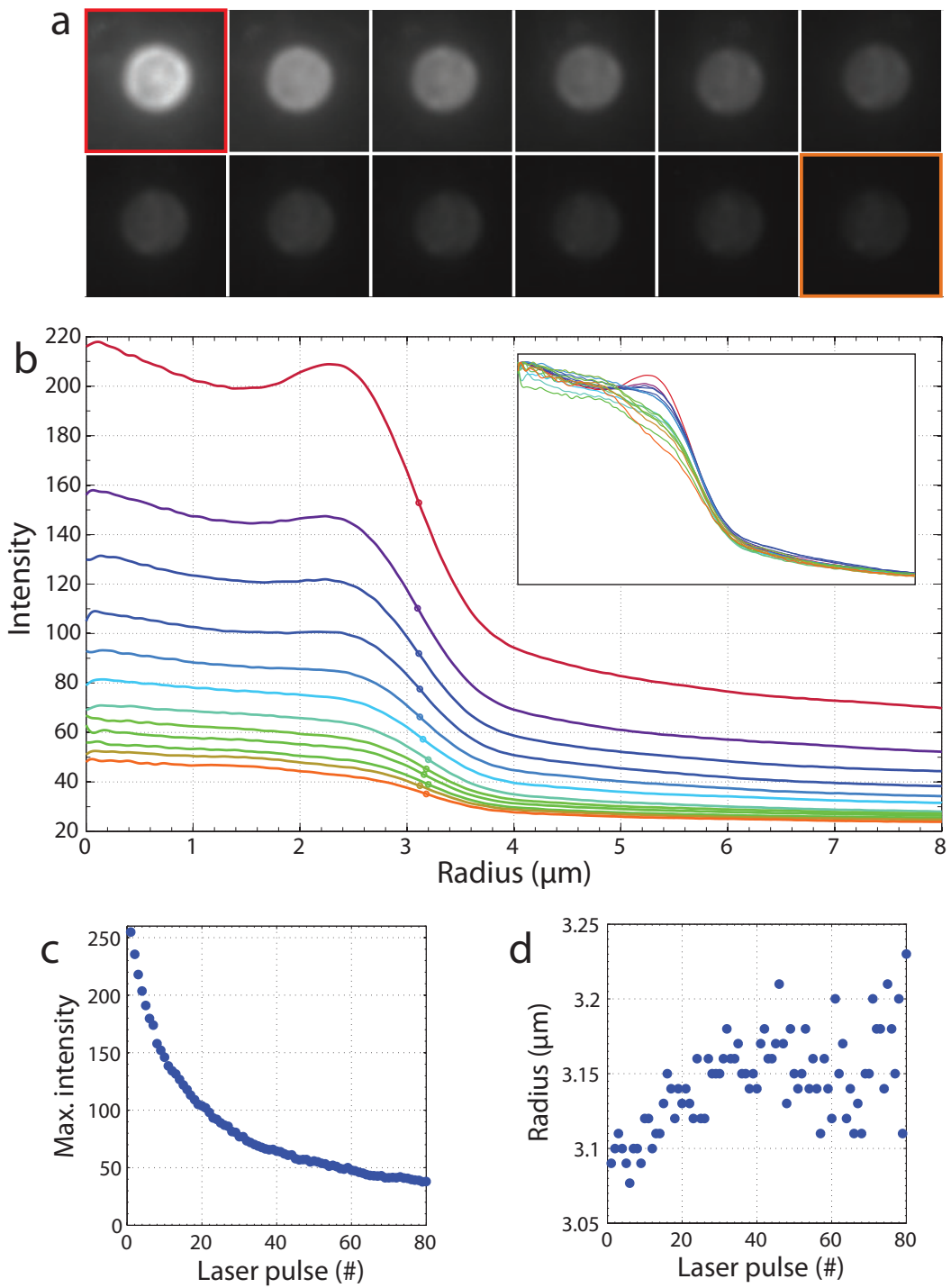

Figure 3.6: Influence of photobleaching on the fluorescence image of a microbubble. a) Bubble images corresponding to the depicted profiles. b) Radial intensity profiles of a single bubble subjected to several laser pulses, resulting in photobleaching and a decrease in fluorescence intensity. The inset shows the normalized profiles. c) Maximum fluorescence intensity as a function of the number of applied laser pulses. d) Corresponding measured radius. After 15 pulses the deviation becomes larger than $1 \%$. 


\section{Out-of-focus error}

In addition to errors caused by the optical configuration, the user can also influence the measured radius when the focus is not determined correctly. In microscopy an image is considered to be in focus when the delineation of objects shows the sharpest transition between the object and its background. Autofocus algorithms operate in a similar fashion by looking at the frequency content of the image [48] and optimizing it for the higher frequencies. In the sizing experiment, this method was applied to the fluorescence bubble images to find the vertical focal plane. The derivative of the transition from the background to the bubble interior was determined and the image with the highest derivative was considered to be in focus. This is shown by the solid red curve in figure 3.7 . When moving away from the focus in either direction, the derivative decreases, indicating that the image is defocused.

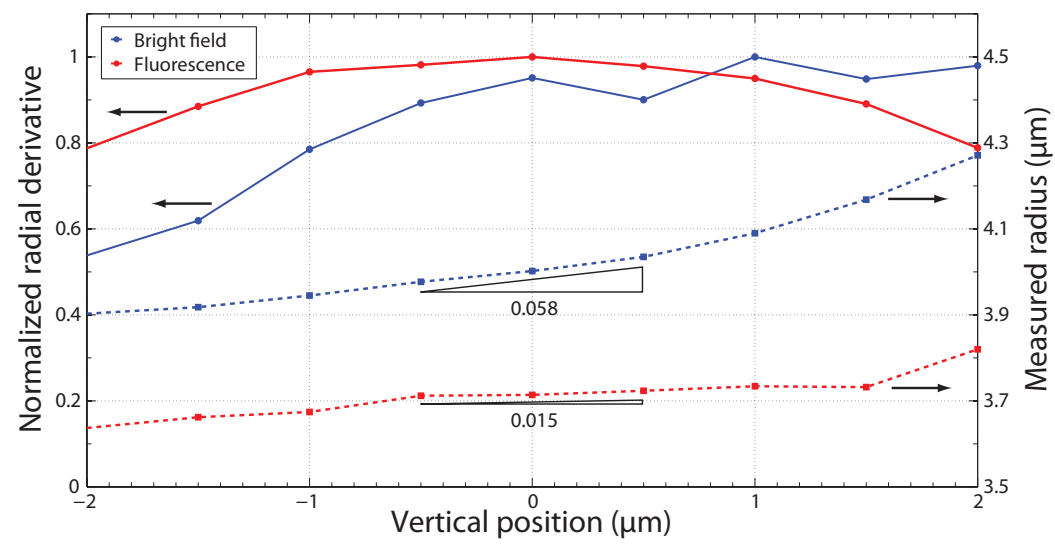

Figure 3.7: Optical contrast as a function of the focal position. The solid red curve shows the derivative of the transition from background to bubble interior for different vertical positions. In bright field, the maximum slope is obtained at a different plane. The dashed lines show the corresponding measured radii. The drawn slopes illustrate the out-of-focus errors.

Applying this method to the bright field bubble images would have resulted in an incorrect focal plane as shown by the solid blue curve in figure B.]. A difference in the apparent focal plane obtained from the bright field images and the focal plane obtained from the fluorescence images was observed in the majority of the experiments. This demonstrates that determining the correct focal position of a bubble in bright field is difficult, since the image with the highest optical contrast does not give the correct focal plane. Well below the focal plane the optical contrast is substantially decreased and several micrometers 
above focus diffraction rings dominate the image, indicating that focus has not been obtained. However, close to the correct vertical position no reliable visual feedback can be obtained from the bright field image of a bubble.

The variation of the measured radius around the focal plane is higher for the bright field images as depicted by the dashed lines in figure 3.0 . In this particular example the slope of the bright field radius is 0.058 and the slope of the fluorescence radius is 0.015 , indicating that the out of focus error is approximately 4 times larger when imaging this bubble in bright field compared to imaging it in fluorescence. The out-of-focus error was determined for all experiments by comparing the radius at the focal position with the radius at $500 \mathrm{~nm}$ above and below this plane. The error is defined as the average of these two values. The results are shown in figure 3.8. In general the error in the bright field measurement is larger than the error in the fluorescence measurement. For smaller bubbles the error increases for both methods. When the method of focusing is not refined enough to have a submicron focusing resolution, the out-of-focus error will be larger, as can be seen from the dashed lines in figure $\mathbf{3 . 7}$.

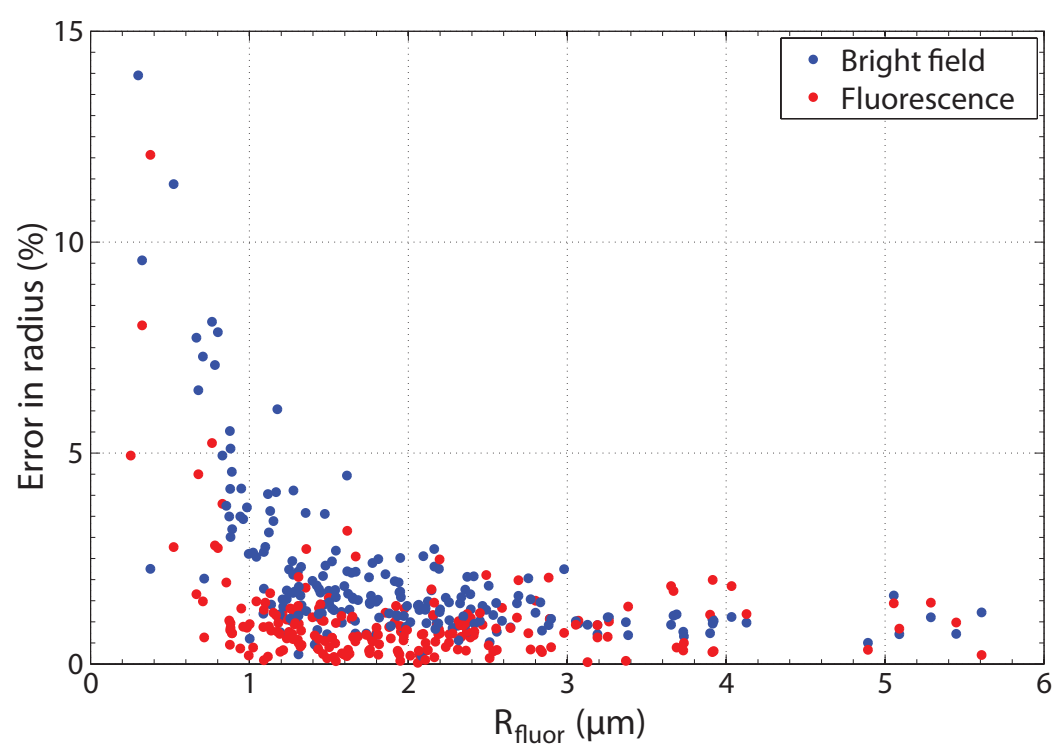

Figure 3.8: Out-of-focus error of the measured radius at $\pm 500 \mathrm{~nm}$. 


\subsection{Results}

The measured bright field radii of the 218 recorded microbubbles are plotted as a function of the measured fluorescence radii in figure B.9la. The results show that the measured radius in bright field is significantly larger than the radius measured in fluorescence. This becomes more evident when the bright field radius is plotted relative to the fluorescence radius in figure $3.9 \mathrm{~b}$. As the bubble size decreases the relative difference can become as large as up to $100 \%$.

No quantitative model is available to predict the relation between the radius in bright field and in fluorescence. From the data points an empirical fit was derived based on an offset between the bright field radius and fluorescence radius. This offset was calculated to have a value of $0.25 \pm 0.08 \mu \mathrm{m}$ and is represented by the dashed lines in figure 3.9. The fit captures the overall trend in the measured data, suggesting that there is a systematic error in the determination of the radius of a microbubble in bright field microscopy. This error is added to the aforementioned errors caused by the optical configuration and incorrect focusing.
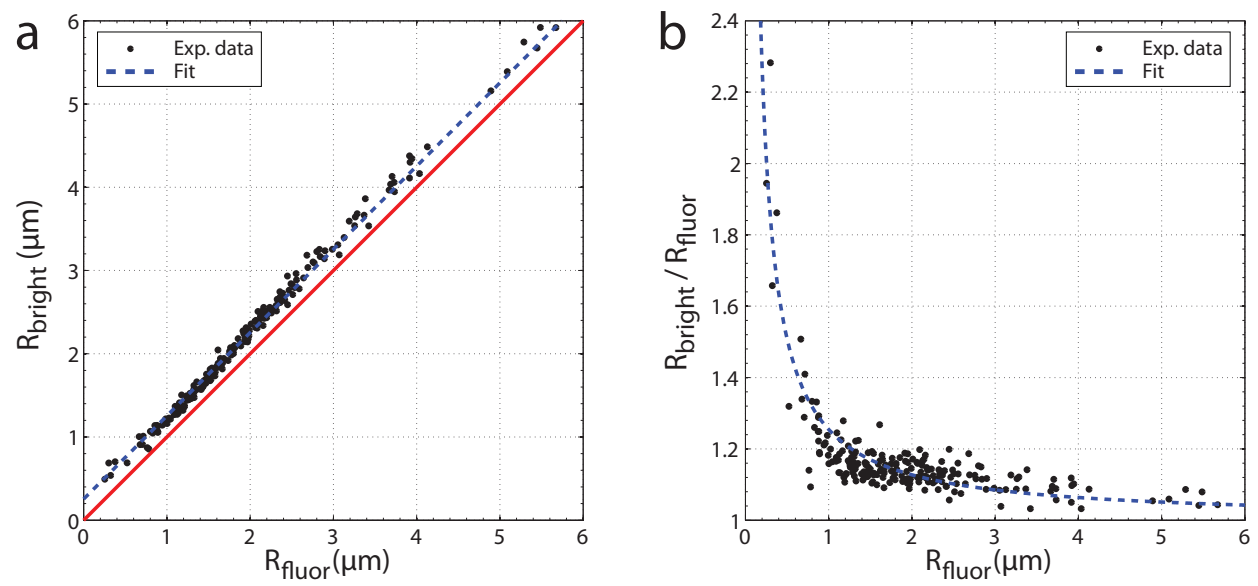

Figure 3.9: a) Comparison of the bubble radii as measured in fluorescence and bright field $(N=218)$. The red line is the line of identity. b) The bright field radius relative to the fluorescence radius. The dashed blue line shows the empirical fit based on an offset of $0.25 \mu \mathrm{m}$. 


\subsection{Application of the results to an existing data set}

The offset in the microbubble radius as determined by bright field microscopy has no influence on the speed and acceleration of the bubble wall during oscillations. However, in the modeling of microbubble dynamics, also the radius itself is of importance. In a recently published paper from Sijl et al. [50] ultrasonically driven, single microbubbles were simultaneously recorded optically and acoustically, with the goal to be able to predict the pressure generated by a microbubble from the measured radial response and vice versa. The ultrasound transducer was calibrated to obtain its receive transfer function and convert the measured voltage signals into pressures. From the optical measurements the pressure as a function of the radius $R(t)$ was derived by using the following equation:

$$
\hat{P}_{s}(r, t-r / c)=\frac{1}{3} \frac{\rho}{r} \frac{\partial^{2}}{\partial t^{2}}\left[R(t)^{3}\right]
$$

where $\rho$ denotes the density of water, $c$ is the speed of sound and $r$ is the distance from the microbubble to the receiver. The pressure has a cubic dependence on the radius, consequently, errors in the measured radius cause an even larger error in the calculated pressure.

A comparison of the measured and calculated pressures displayed significant differences and variation between the two values, which were attributed to attenuation of the acoustic signal by the cellulose capillary tube, a possible error in the transducer calibration and/or an error in the radius determination of the microbubbles.

By applying the empirical model derived for the bright field radius, the calculated pressures can be corrected. The results of this correction are shown in figure 3.10 . The data points are shifted downwards, due to the offset in the bubble radius. The histograms show the difference between the measured and calculated pressures. Without correction the mean absolute error is $-1.1 \mathrm{~Pa}$. After correction the mean absolute error is $-0.3 \mathrm{~Pa}$. In both cases the standard deviation is $1.8 \mathrm{~Pa}$. 

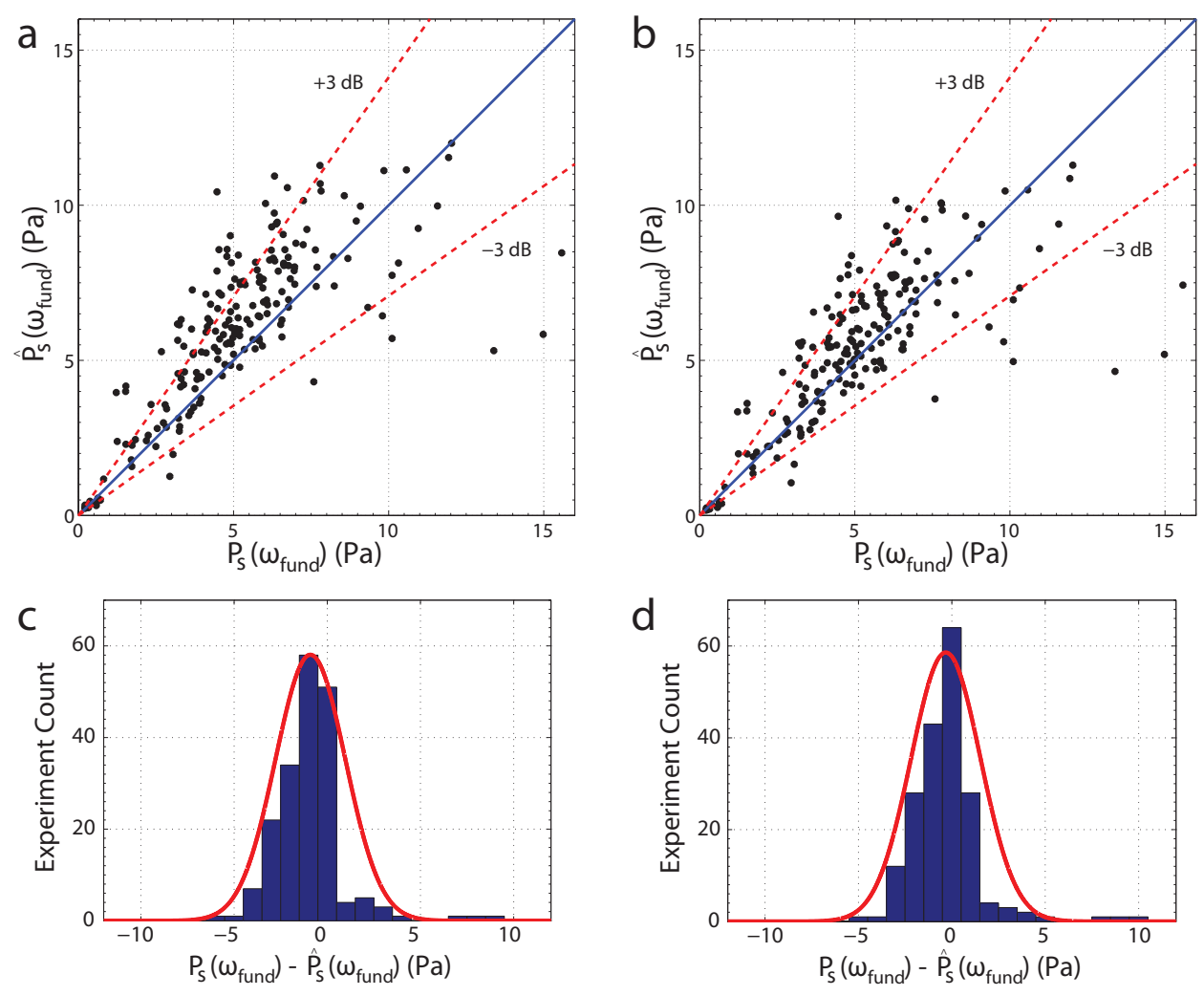

Figure 3.10: A comparison between the amplitude of the acoustic bubble response at the driving frequency as measured by the receive transducer $\left(P\left(\omega_{\text {fund }}\right)\right)$ and the acoustic response as predicted from the measured $\mathrm{R}(\mathrm{t})$ curve $\left(\hat{P}\left(\omega_{\text {fund }}\right)\right)$. a) The original data from Sijl et al. and b) the corrected data. $c, d)$ Histograms of the data plotted in a and b.

\subsection{Conclusions and discussion}

Optical sizing of ultrasound contrast agent microbubbles with a radius approaching the wavelength of light is difficult, because transmitted light microscopy produces a complex bubble image at these length scales. In fluorescence microscopy, the final bubble image does not suffer from diffraction or scattering of the incident light. This is reflected in the final bubble image, which reveals even the nanometer-sized arrangement of the fluorescent material on the bubble shell. The optical system is still limited by its numerical aperture and the wavelength of the light, however, the intensity profile is much simpler and the optical system can be approximated by a diffraction-limited thin lens without 


\subsection{CONCLUSIONS AND DISCUSSION}

the need to account for near field diffraction or scattering.

By using the fluorescence bubble image as a calibration for the bubble imaged in bright field, an empirical relation was found to correct the bubble radius measured in bright field. A systematic offset of $0.25 \mu \mathrm{m}$ was found, independent of the microbubble size. Despite a calibration of the bright field bubble radius, error sources cannot be ruled out in future experiments. The exact illumination method will depend on the experimental setup and the required light intensities. In high-speed imaging, the limited exposure time can require the use of a high read-out gain, leading to image degradation and a loss of resolution and signalto-noise ratio.

Even if the experimental parameters are kept constant, users will most likely not be able to focus at the correct focal plane for each experiment. Users may also consider different focal planes to be the correct one. This can result in systematic as well as random errors. Intra-observer and inter-observer variability have to be considered. A study by Sennoga et al. [5I] resulted in an intra-observer variability of $8 \%$ and an inter-observer variability of $3.8 \%$ for the sizing of SonoVue ${ }^{\mathrm{TM}}$ microbubbles, demonstrating that the comparison of optical studies of microbubble dynamics performed by different researchers in different labs is not straightforward. This precaution also applies to the corrected results from Sijl et al. [50].

Furthermore, during an experiment microbubbles will reside at the top of a container, such as a capillary or Opticell ${ }^{\mathrm{TM}}$. Ultrasound is then applied from the bottom or side of a water bath and a vertically aligned microscope objective is used to optically capture the bubble behavior. During ultrasonic excitation, the bubble does not just undergo radial excursions, it will also translate as shown by Vos et al. [52, 53]. In the expansion phase, the bubble will move downwards as it pushes itself away from the surface. Consequently, during the oscillations, the center of the bubble will move with respect to the focal plane of the objective. The resulting out-of-focus error depends on the exact amplitude of oscillation. When the amplitude is kept below one micron, the error will be on the order of 5-10\%.

Even when a complete theoretical description of the intensity profile of a microbubble would be available and validated, each and every microbubble will still require a calibration measurement. Since the focal plane is not known a priori, a bubble that is out of focus can have an intensity profile similar to a bubble that is in focus but with a different size. Therefore fluorescence imaging is a good calibration method, since it not only provides the radius of the bubble, the position of the focal plane is obtained by using a standard focus algorithm. 


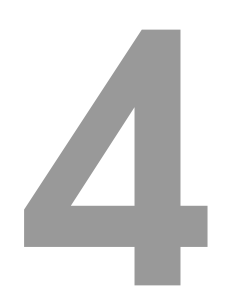

\section{High-speed fluorescence imaging of the US-triggered release from liposome-loaded microbubbles}

The ultrasound-triggered release of fluorescently labeled liposomes from liposome-loaded microbubbles was characterized using high-speed fluorescence imaging at 100000 frames per second and compared to the release of fluorescently labeled phospholipids from unloaded microbubbles. Over 700 bubbles with a radius ranging from 2 to $6 \mu \mathrm{m}$ were insonified at a driving frequency of $1 \mathrm{MHz}$ and at acoustic pressures varying from 20 to $100 \mathrm{kPa}$ and pulse lengths of 10, 100 and 1000 cycles. Below the threshold of release, rearrangement of the fluorescent shell material at the bubble wall occurs. Above the threshold two distinct types of release were observed both for unloaded and liposomeloaded microbubbles: the release of individually resolved micron-sized particles and the release of a mist of submicron particles. The two release mechanisms occur independently from each other and result in different release velocities and release distances of the expelled fluorescent material. 


\subsection{INTRODUCTION}

\subsection{Introduction}

Coated microbubbles are widely employed for several decades in diagnostic medical ultrasound (US) imaging as contrast agents to improve the blood-to-tissue contrast in organ perfusion imaging [1, 54]. The technique offers real-time, non-invasive imaging at a low cost and the use of diagnostic ultrasound in combination with contrast agent microbubbles has proven to be safe for humans [55].

Even before the use of microbubbles in ultrasound imaging, therapeutic applications of ultrasound were considered by means of enhanced perfusion caused by mild hyperthermia [56]. Microbubbles were introduced in this field by lowering the cavitation threshold in sonothrombolysis [57]. Since then the number of therapeutic applications of ultrasound contrast agents (UCAs) has increased rapidly $[7,58,59]$. Ultrasound-triggered uptake by vascular endothelial cells can be enhanced by oscillations of co-administered microbubbles to improve drug and gene delivery $[60,6]]$. Likewise, microbubble oscillations can aid therapeutic agents to cross the blood-brain barrier in order to treat central nervous system disorders [62].

Bubble-enhanced drug delivery can also be achieved by incorporating a therapeutic agent into the bubble shell material or its interior. Various designs of drug-loaded microbubbles exist for both soft-shelled and hard-shelled agents [ [ $\mathrm{Z}$, 8]. A high payload is achieved by incorporating lipophilic drugs into the core of polymeric microcapsules [23, 41, 63]. The thick polymer shell of microcapsules $(20-100 \mathrm{~nm})$ can also be used to incorporate drugs, as shown by Hauff et al. [64]. By using double emulsion technology, not only lipophilic but also hydrophilic drugs can be incorporated into hard-shelled agents. Hauff et al. [64] were able to produce microcapsules containing plasmid DNA and demonstrated an effect on tumor growth and gene delivery in an animal model.

The thin, monolayer shell of phospholipid-coated microbubbles does not allow for a large amount of drugs to be incorporated directly into the bubble shell or bubble core. Unger and co-workers [65, 66] demonstrated such a system fifteen years ago by adding an oil to the phospholipid monolayer to increase the thickness of the shell, however, only hydrophobic drugs are compatible with this configuration. To enhance the payload of soft-shelled agents drugcarrying liposomes can be linked to the microbubble surface [9, 10, 60-70]. Vandenbroucke et al. [69] demonstrated that efficient gene silencing can be achieved in carcinoma cells through ultrasonic exposure of microbubbles loaded with siRNA-liposome complexes. Lentacker et al. [9] showed that the uptake of doxorubicin (DOX) in melanoma cells was significantly enhanced by insonation of microbubbles loaded with DOX-carrying liposomes. 
Although the therapeutic effect has been demonstrated, the physical mechanism of local drug delivery using drug-loaded microbubbles has not been fully clarified. An essential step in the process of ultrasound-triggered drug delivery is the release of the therapeutic agent from the bubble surface. In the case of a liposome-loaded bubble, two delivery methods can be distinguished. First, the release of the therapeutic agent incorporated into the liposome. This can be achieved by disrupting the liposome while it is still attached to the bubble surface or when the liposome is first released and subsequently disrupted. Secondly, the release of the intact liposome from the bubble surface, which acts as the therapeutic agent. The second method has been demonstrated by DeTemmerman et al. [70]: for transfection of mRNA into dendritic cells, the mRNA was required to be linked to a lipoplex. The different nature of the release will rely heavily on the intrinsic physical driving mechanisms of release. Consequently, it will also strongly depend on the applied ultrasound parameters, such as pressure, frequency and the number of cycles.

Here we present a parametric study into the controlled release from liposomeloaded microbubbles at a fixed insonation frequency of $1 \mathrm{MHz}$ for a peaknegative pressure varying from $20-100 \mathrm{kPa}$ and a pulse duration of 10,100 and 1000 cycles. As the bubbles oscillate at the frequency of the applied ultrasound, the release will take place on a timescale of tens of nanoseconds. The subsequent transport of released material into the surrounding liquid will be on the timescale typically three orders slower, i.e. tens to hundreds of microseconds. To visualize these rapid transport phenomena and to quantify the release and transport dynamics, high-speed imaging at a microseconds timescale is required. Furthermore, the drugs or drug-carrying liposomes, with a size of several nanometers to $200 \mathrm{~nm}$, respectively, are at a scale too small to be resolved with transmitted light microscopy. Fluorescent labeling of the liposomes or drugs is then required to achieve sufficient optical contrast.

Fluorescence imaging on a timescale of microseconds is challenging, since the available exposure time to collect the fluorescence emission is limited. Secondly, the total number of fluorescent molecules is restricted by the small volume of the liposome, hence, the total number of fluorescence emission photons is limited. To maximize the fluorescence signal, the excitation intensity has to be sufficient to ensure that all molecules are excited in each frame. Such illumination intensity can only be achieved by employing a laser as fluorescence excitation light source.

Through the use of a continuous wave (CW) laser as the excitation illumination for fluorescence microscopy, we have been able to visualize liposome-loaded microbubbles on a microseconds timescale. This technique has been combined with a high-speed camera and an acoustical setup to perform time-resolved 
high-speed fluorescence microscopy of US-triggered release. As a first step, we have visualized the process of controlled release at a frame rate of 100000 frames per second ( $\mathrm{fps}$ ). The goal of this study is to distinguish between the various release mechanisms that occur when liposomes are released as a result of US-driven microbubble oscillations.

\subsection{Materials and methods}

\subsubsection{Microbubbles}

\section{Microbubble systems}

Four samples were prepared with different configurations of the bubble system. Two samples of unloaded microbubbles were prepared ( $A$ and $B$ ) and two samples of liposome-loaded microbubbles ( $C$ and $D)$, as depicted in figure 4.7 . Sample A was devised to visualize the shedding of phospholipids from the bubble shell and consisted of a non-functionalized microbubble labeled with Dil. These microbubbles yielded a high fluorescence signal and were relatively simple to construct. The incorporation of Dil in the phospholipid monolayer is based on hydrophobic interactions and the hydrophobicity prevents the Dil molecules to diffuse out of the bubble shell. However, microbubble oscillations might lead to the creation of Dil clusters, which would interfere with the fluorescence signal of the shedding of phospholipids. Therefore sample B was constructed, consisting of functionalized microbubbles labeled with a maleimide-functionalized Alexa dye. The strong binding force between the phospholipids and the fluorescent markers $(\sim 300 \mathrm{pN})$ ensured that any measured fluorescence signal would correspond to the location of phospholipids. A drawback of this configuration is its relatively low fluorescence yield, which required the use of additional read-out gain on the camera, resulting in substantial noise levels and a reduced signal-to-noise ratio (SNR).

Sample $\mathrm{C}$ was constructed to visualize the release of liposomes from the microbubble shell and consisted of functionalized microbubbles loaded with functionalized liposomes with Dil membrane labeling. These loaded microbubbles yielded similar fluorescence signal levels as sample A, however the incorporation of the dye molecules into the lipid bilayer is based on hydrophobic interactions. Sample D was devised as a control system for sample C, consisting of functionalized microbubbles loaded with functionalized liposomes with encapsulated fluorescent dextran. As with sample $B$, the fluorescence yield was substantially lower, compared to the Dil labeled liposome-loaded microbubbles. 


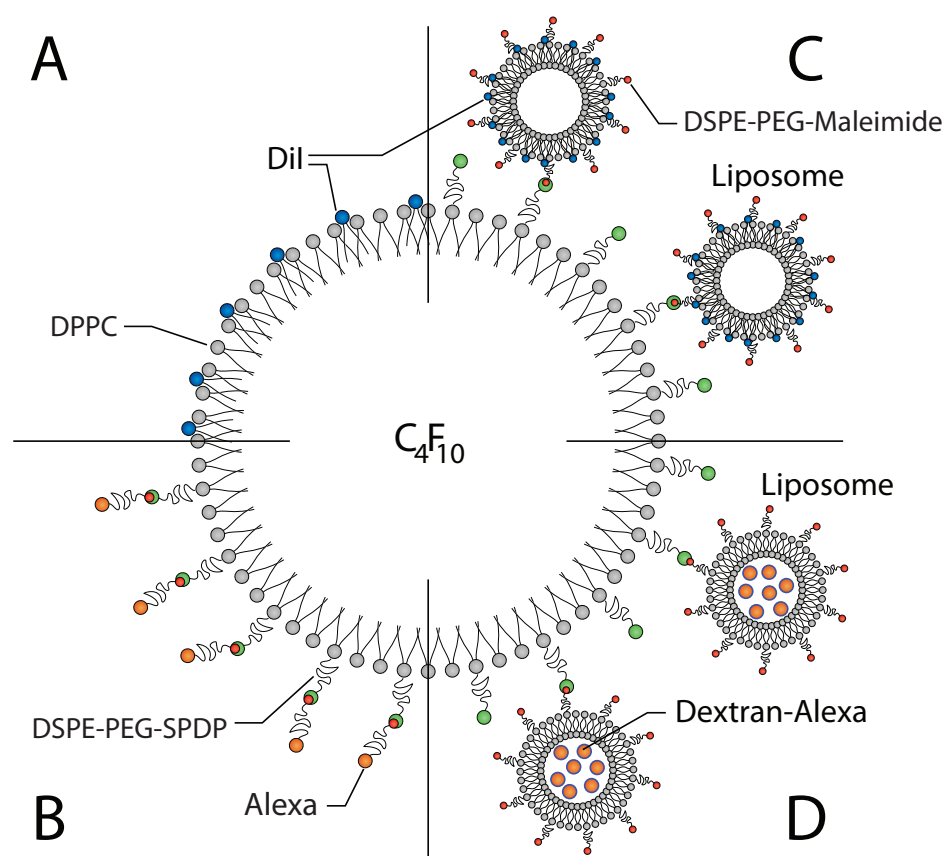

Figure 4.1: Schematic representation of the four configurations of bubble systems.

\section{Microbubble formulations}

Microbubbles were prepared by mixing 1,2-dipalmitoyl-sn-glycero-3-phosphocholine (DPPC) (Lipoid, Ludwigshafen, Germany) and either 1,2-distearoylsn-glycero-3-phosphoethanolamine-N-[PDP(polyethylene glycol)-2000] (DSPEPEG-SPDP) for functionalized bubbles or 1,2-distearoyl-sn-glycero-3-phosphoethanolamine-N-[methoxy (polyethylene glycol)-2000 (DSPE-PEG) (both Avanti polar lipids, Alabaster, $A L$ ) for non-functionalized bubbles in a molar ratio of 65:35 in chloroform. For fluorescent labeling of the microbubble membrane, the lipophilic dye Dil (Sigma-aldrich, Bornem, Belgium) was added to the lipid mixture. After chloroform evaporation, the lipid film was dissolved in a 1:2:7 glycerine-propyleneglycol- $\mathrm{H}_{2} \mathrm{O}$ mixture to obtain a clear solution with a final lipid concentration of $4 \times 10^{-4} \mathrm{mmol} / \mathrm{mL}$. This lipid solution was aliquotted in $2.5 \mathrm{~mL}$ chromatography vials of which the headspace was filled with perfluorobutane gas (F2 chemicals, Preston, UK). Finally, bubbles were obtained by high speed shaking of the chromatography vial in a Capmix ${ }^{\text {TM }}$ device (3 M-ESPE, Diegem, Belgium) for $15 \mathrm{~s}$.

Maleimide-functionalized liposomes were prepared by mixing DPPC, 1,2distearoyl-sn-glycero-3-phosphoethanolamine- $\mathrm{N}$-[maleimide(polyethylene glycol)- 
2000] (ammonium salt) (DSPE-PEG maleimide) (Laysan Bio Inc, Arab, AL), cholesterol and Dil, all dissolved in chloroform in a round-bottom flask at a 49:15:35:1 molar ratio and a final lipid concentration of $16 \mathrm{mg} / \mathrm{mL}$ lipids. The chloroform was evaporated and the remaining lipid film was hydrated with distilled water. The liposomes were sized by extrusion through a $200 \mathrm{~nm}$ polycarbonate filter using a mini-extruder at $60^{\circ} \mathrm{C}$ (Avanti Polar Lipids, Alabaster, $A L)$.

To prepare functionalized liposomes with encapsulated fluorescent dextran, liposomes were produced as described above, by preparing a mixture of DPPC, DSPE-PEG maleimide and cholesterol in a 49:15:35 molar ratio in chloroform, at a final lipid concentration of $16 \mathrm{mg} / \mathrm{mL}$ lipids. After chloroform evaporation, the lipid film was hydrated with a $5 \mathrm{mg} / \mathrm{mL}$ solution of dextran-Alexa Fluor ${ }^{\circledR}$ $546(\mathrm{MW}=10000)$ (Molecular Probes ${ }^{\circledR}$, Invitrogen, Merelbeke, Belgium). The resulting liposomes were sized by extruding through a $200 \mathrm{~nm}$ polycarbonate filter using a mini-extruder at $60^{\circ} \mathrm{C}$. Excess extra-liposomal fluorescent dextran was removed by three ultracentrifugation washing steps.

Microbubbles were covalently loaded with maleimide-functionalized liposomes by adding $100 \mu \mathrm{L}$ of the liposome dispersion to $900 \mu \mathrm{L}$ of the bubble lipid mixture in a $2.5 \mathrm{~mL}$ chromatography vial. After filling the headspace with perfluorobutane gas, the vial was shaken for $15 \mathrm{~s}$ in a CapMix ${ }^{\text {TM }}$ device. This results in the formation of liposome-loaded microbubbles. Similarly, bubbles could be covalently loaded with Alexa Fluor ${ }^{\circledR} 546 \mathrm{C}_{5}$ Maleimide (Molecular Probes ${ }^{\circledR}$, Invitrogen, Merelbeke, Belgium) by adding an aliquot of a $1 \mathrm{mg} / \mathrm{mL}$ solution of this dye to the bubble lipid mixture in a chromatography vial, filling the headspace with perfluorobutane gas followed by high-speed shaking of the vial in a CapMix ${ }^{\text {TM }}$ device for $15 \mathrm{~s}$.

\section{Optical characterization}

When observed in transmitted light microscopy, the unloaded and liposomeloaded microbubbles have a similar appearance, as shown in figure 4.2 . However, in fluorescence the difference in the distribution of fluorescent material over the shell of the bubble becomes visible. The unloaded microbubble displays a homogenous distribution of fluorescent material, whereas the fluorescence on the liposome-loaded microbubbles is arranged heterogeneously. Figure $4.2 \mathrm{c}$ and $f$ show the size distribution for the measured population of unloaded microbubbles $(3.3 \pm 0.7 \mu \mathrm{m})$ and liposome-loaded microbubbles $(3.8 \pm 0.7 \mu \mathrm{m})$. During the experiments, the microbubbles were selected on their approximate size, in order to achieve a similar size distribution for all bubble configurations. The depicted distribution is therefore not a representation of the total microbubble 
population.
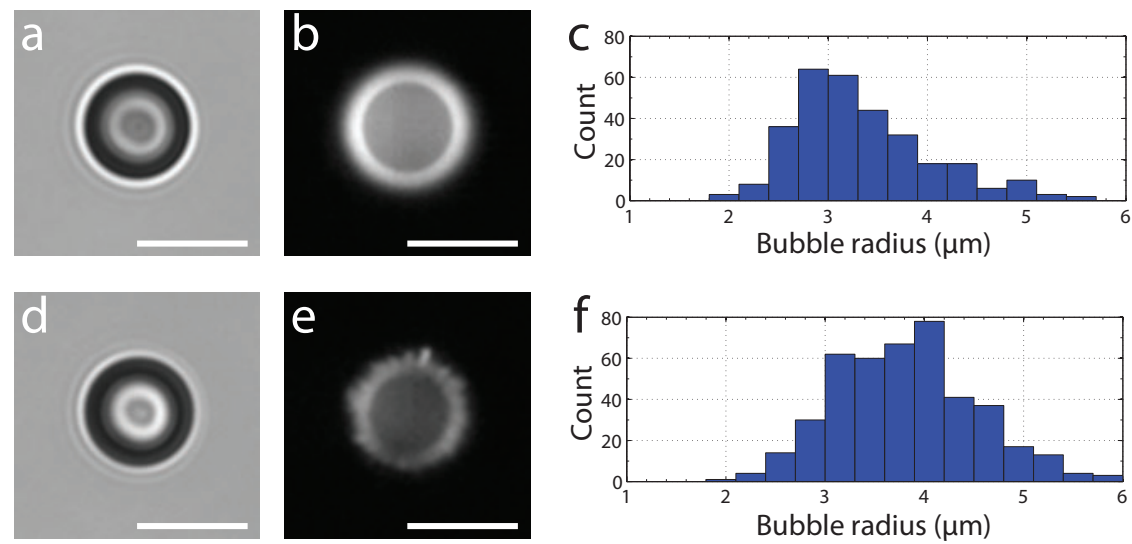

Figure 4.2: a,b) Unloaded microbubble (sample A) in bright field and fluorescence. c) Size distribution of measured unloaded bubbles. $d, e$ ) Liposome-loaded microbubble (sample C) in bright field and fluorescence. f) Size distribution of measured liposomeloaded bubbles. Scale bars represent $10 \mu \mathrm{m}$.

As shown in figure 4.3, the distribution of liposomes can result in various nanoconstructs when there is an extended period of time between preparation and observation. These structures are similar to those described by Borden and co-workers [42, 71, 72]. Mostly dark domains surrounded by a bright matrix were observed in large microbubbles (radius $>10 \mu \mathrm{m}$ ), indicating that phase separation of the different phospholipids had taken place. Figure $4.3 \mathrm{~d}$ shows a loaded microbubble with a structure of fluorescent material covering a large area outside the microbubble. Presumably, the bubble had shrunk over time, shedding the excess shell material into a structure of PEGylated strands.
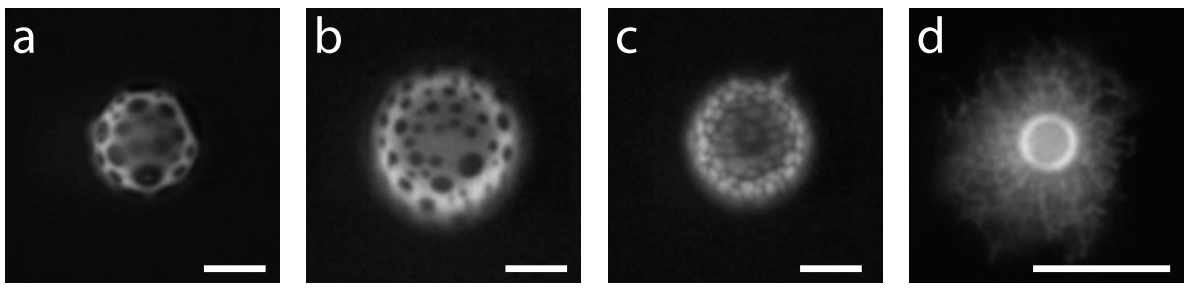

Figure 4.3: a-c) Fluorescence images of large liposome-loaded microbubbles (sample C) showing phase separation of the different phospholipids. d) Shrunken microbubble (sample C) with strands of excess fluorescence shell material. Scale bars represent $10 \mu \mathrm{m}$. 


\subsubsection{High-speed fluorescence imaging setup}

The Opticell ${ }^{\text {TM }}$ containing the microbubbles was placed in a water bath, located underneath an upright fluorescence microscope setup, depicted in figure 4.4. The microscope was equipped with a $40 \times$ water-immersion objective (NA $=0.8$; Olympus, The Netherlands). To achieve a high fluorescence signal, a CW laser (5 W@532 nm; Cohlibri; Lightline, Germany) was employed for fluorescence excitation of the fluorescent label. The laser light was gated using a acousto-optic modulator (AOM) (AOTF.nC-VIS; AA Optoelectronic, France) to generate a single pulse with a duration of $6 \mathrm{~ms}$ to minimize heating of the sample. Bright field illumination was produced by a halogen light source (KL1500LCD, Schott, Germany). The intensity was adjusted to a level that allowed for monitoring of the bubble position during the high-speed fluorescence recordings.

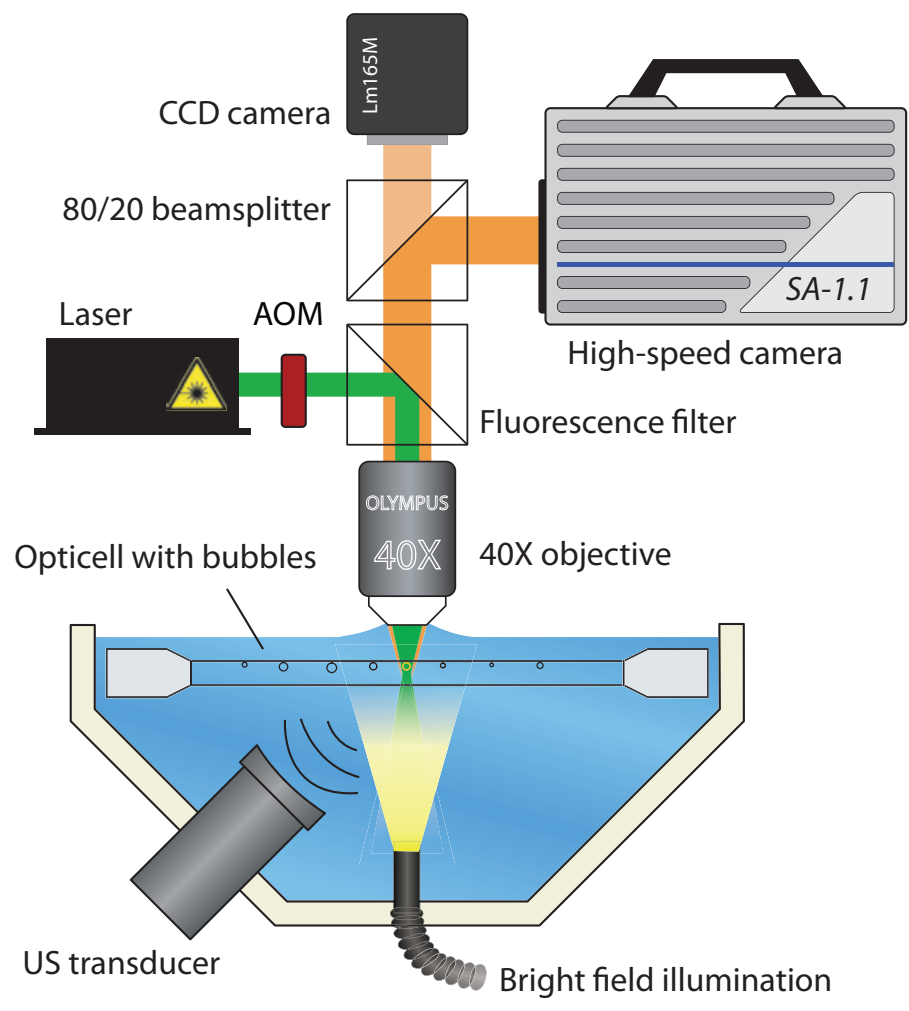

Figure 4.4: Schematic representation of the experimental setup. The Opticell ${ }^{\mathrm{m}}$ containing microbubbles is placed in a water bath, located underneath a fluorescence microscope setup with a high-speed camera operating at $100 \mathrm{kfps}$ and a CCD-camera for simultaneous video rate bright field recordings. 
High-speed fluorescence images of the liposome-loaded microbubbles were recorded with a CMOS-based high-speed camera (SA-1.1; Photron Ltd,UK), operating at $100 \mathrm{kfps}(320 \times 160$ pixels $)$, starting $1 \mathrm{~ms}$ prior to ultrasound excitation for a duration of $6 \mathrm{~ms}$ ( 600 frames). The microscope was fitted with a $80 / 20$ beamsplitter to allow for simultaneous video rate bright field recordings with a larger field of view at $15 \mathrm{fps}$ using a highly sensitive CCD-based camera (LM165M; Lumenera Corporation, Canada). Bright field recordings were started one second before ultrasound excitation for a duration of 10 seconds to monitor the bubble displacement and possible bubble shrinkage.

The microbubbles were insonified by a single, sine-wave ultrasound pulse with a duration of 10,100 or 1000 cycles at an ultrasound frequency of $1.0 \mathrm{MHz}$ at five peak-negative pressures $(20,40,60,80$ and $100 \mathrm{kPa})$ using a focused, single element transducer (C302; Panametrics, USA). The pressures generated by the transducer were calibrated with a $2 \mathrm{~mm}$ PVDF needle hydrophone (Precision Acoustics, UK).

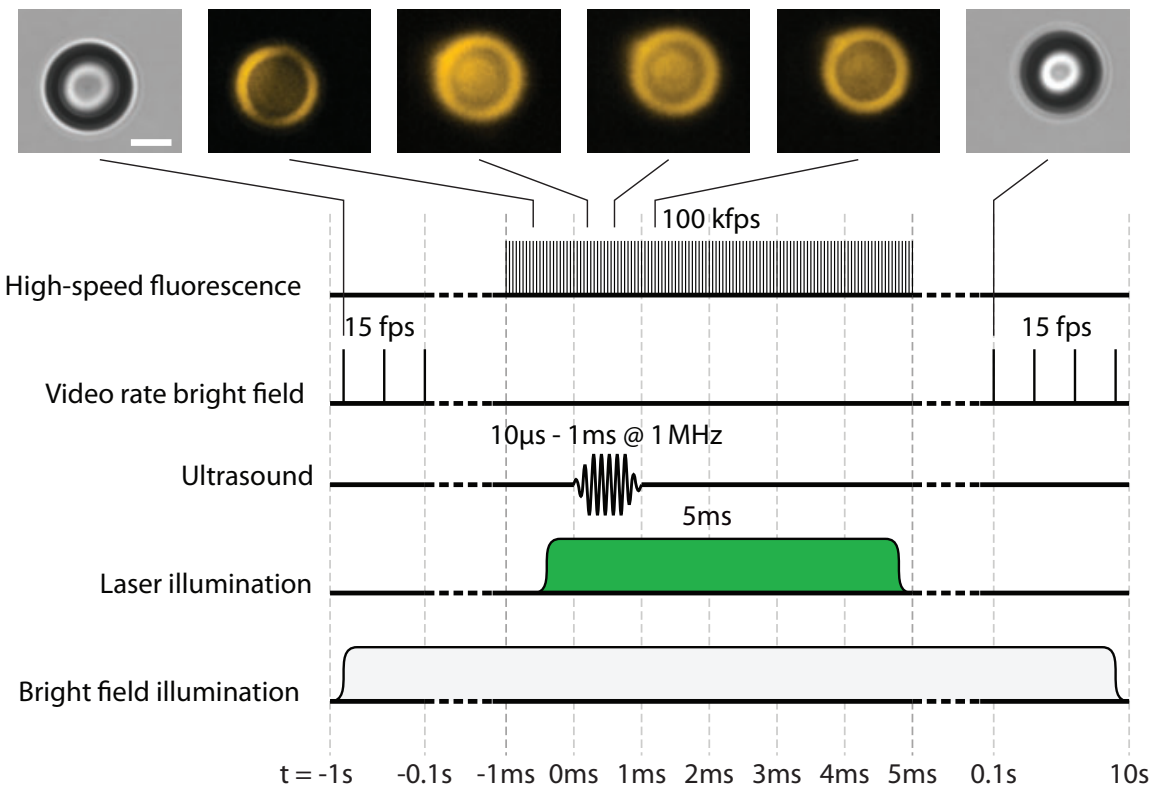

Figure 4.5: Timing diagram of a single experiment. A bright field and fluorescence image show the bubble before and after insonation. Two fluorescence frames recorded during insonation show a blurred shell, because the frame rate is an order of magnitude lower than the ultrasound frequency. Scale bar represents $5 \mu \mathrm{m}$.

The timing diagram of a single experiment is given in figure 4.5. Several bright field and fluorescence sample frames are included. The frame rate of 


\subsection{MATERIALS AND METHODS}

the bright field recordings did not allow for imaging during insonation. The frame rate of the high-speed fluorescence recordings is one order of magnitude lower than the ultrasound frequency $(1 \mathrm{MHz})$, causing the shell of an oscillating bubble to be motion-blurred. The release from the bubble surface on the timescale and length scale of the bubble oscillations cannot be resolved by our measurements, however, the transport into the surrounding liquid on a micrometer length scale, that takes place on a timescale of tens to hundreds of microseconds was fully captured by the camera. The maximum record length of the fluorescence recordings was limited to several milliseconds, due to photobleaching of the fluorescent dyes, which was caused by the high intensity laser illumination.

\subsubsection{Data analysis}

Microbubbles display a strong resonance behavior as a function of the applied ultrasound frequency. Since the amplitude of oscillation was expected to be an important parameter in the process of release, the insonation frequency was kept constant and the microbubbles were selected within a limited size distribution, to minimize the influence of the bubble size on the amplitude of oscillation and possible release.

Nevertheless, the amplitude of oscillation of a liposome-loaded microbubble will differ from an unloaded microbubble and the high-speed fluorescence recordings do not provide information on the exact oscillation amplitude. Luan et al. [73] showed that the oscillation amplitudes for liposome-loaded bubbles are approximately a factor 2 lower compared to unloaded bubbles, however, only a minor difference in the resonance frequencies for both microbubble systems was observed. Hence, no significant influence of microbubble size on the results for different configurations is expected in this study.

For every combination of bubble configuration, acoustic pressure and pulse duration, a minimum of 10 microbubbles was recorded. This parametric study resulted in a total number of 737 experiments, each comprised of a high-speed fluorescence recording and a low-speed bright field recording. From the bright field images, the bubble size and position before and after insonation were obtained. The high-speed fluorescence recording provided detailed information about the release and subsequent transport of fluorescent material. First, the type of release was established. Then, several quantities were determined, such as the delay between the onset of ultrasound and the release, the maximum distance to which fluorescent material was released and the initial velocity at which the release takes place. 
At high acoustic pressures, bubbles were displaced during actuation due to primary and/or secondary acoustic radiation forces. In these cases, released fluorescent material was displaced as well, as a result of a change in the flow field compared to the situation where the bubble would be stationary. The contribution to the transport of fluorescent material due to bubble displacement was subtracted from the measured value.

Samples B and D were devised as control systems for sample A and C, respectively. The results showed good agreement between the two unloaded samples and between the two loaded samples. Therefore the results for sample $A$ and $B$ and for sample $C$ and $D$ are binned in the following.

\subsection{Results}

\subsubsection{Rearrangement of fluorescent shell material}

At the frame rate of the high-speed recordings of this study, the microbubble oscillations could not be resolved. However, at the microseconds timescale of the fluorescence recordings, movement of fluorescent material over the bubble surface was visualized. As the bubble oscillates radially, both unloaded and liposome-loaded bubbles showed lateral rearrangement of fluorescence in the bubble shell for both fluorescent labels. No correlation was found between the movement of fluorescent material and the release of fluorescent material, as these movements were observed for all ultrasound settings.

The movements were typically non-uniform and appeared random and chaotic as various parts of the shell would display different flow directions and/or velocities. Two examples in which the observed movements were ordered and occurred at relatively low velocities, are given in figure 4.6. The first example shows the displacement of a spot of high concentration of fluorescent material over the bubble shell, whereas the second example shows rotational movement. Four connecting vortices were observed and one of them is highlighted in the image sequence.

Before or after ultrasound excitation, no movement was observed, indicating the bubble oscillations are the driving force behind the flow of shell material. Measured velocities are on the order of one centimeter per second, which is three orders of magnitude faster than reported values for the diffusion rate of lipids in a monolayer [74]. 


\subsection{RESULTS}

a

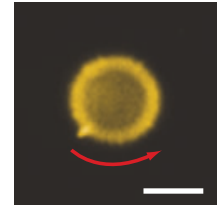

$\mathrm{t}=0 \mu \mathrm{s}$

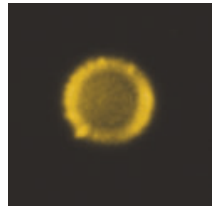

$40 \mu \mathrm{s}$

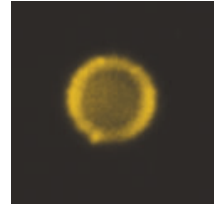

$80 \mu \mathrm{s}$

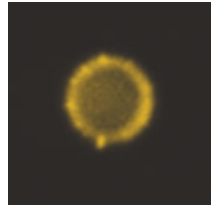

$120 \mu \mathrm{s}$

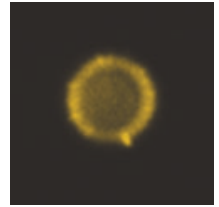

$160 \mu \mathrm{s}$

b
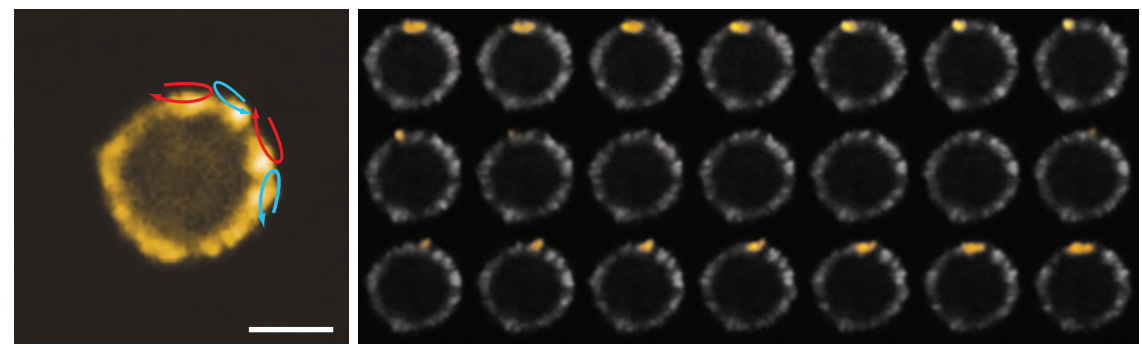

Figure 4.6: Rearrangement of fluorescent material over the bubble shell. a) A spot of high concentration moves over the bubble surface (sample C, $40 \mathrm{kPa}, 1000$ cycles). b) Rotational flow on the bubble surface (sample C, $100 \mathrm{kPa}, 1000$ cycles). The top vortex is highlighted in the image sequence (10 $\mu$ s inter-frame time). Scale bars represent $5 \mu \mathrm{m}$.

\subsubsection{Release observations}

Two distinct types of release from unloaded and loaded microbubbles were observed. The first type can be described as the release of vesicles with a size on the order of several hundreds of nanometers to a micrometer that can be resolved by the optical system. These vesicles detach from the microbubble shell over the course of the microbubble actuation and can be traced as single particles in the flow field around the bubble. For unloaded bubbles, vesicles are clustered phospholipids that have formed a micelle or liposome. For loaded bubbles, vesicles can be clusters of liposomes or small parts of the bubble shell with liposomes still attached.

The second type of release can be described as the release of a bulk of molecular to submicron particles, too small to be resolved individually, consequently observed as a mist emerging from the microbubble surface. This type of release takes place on a much shorter timescale, appearing as the instantaneous release of a large part of the fluorescent shell material, being expelled radially outwards. For unloaded bubbles, the mist consists of nanometer-sized clusters of phospholipids. For loaded bubbles, the mist presumably consists of single liposomes. Both types of release are depicted schematically in figure 4.0 . 


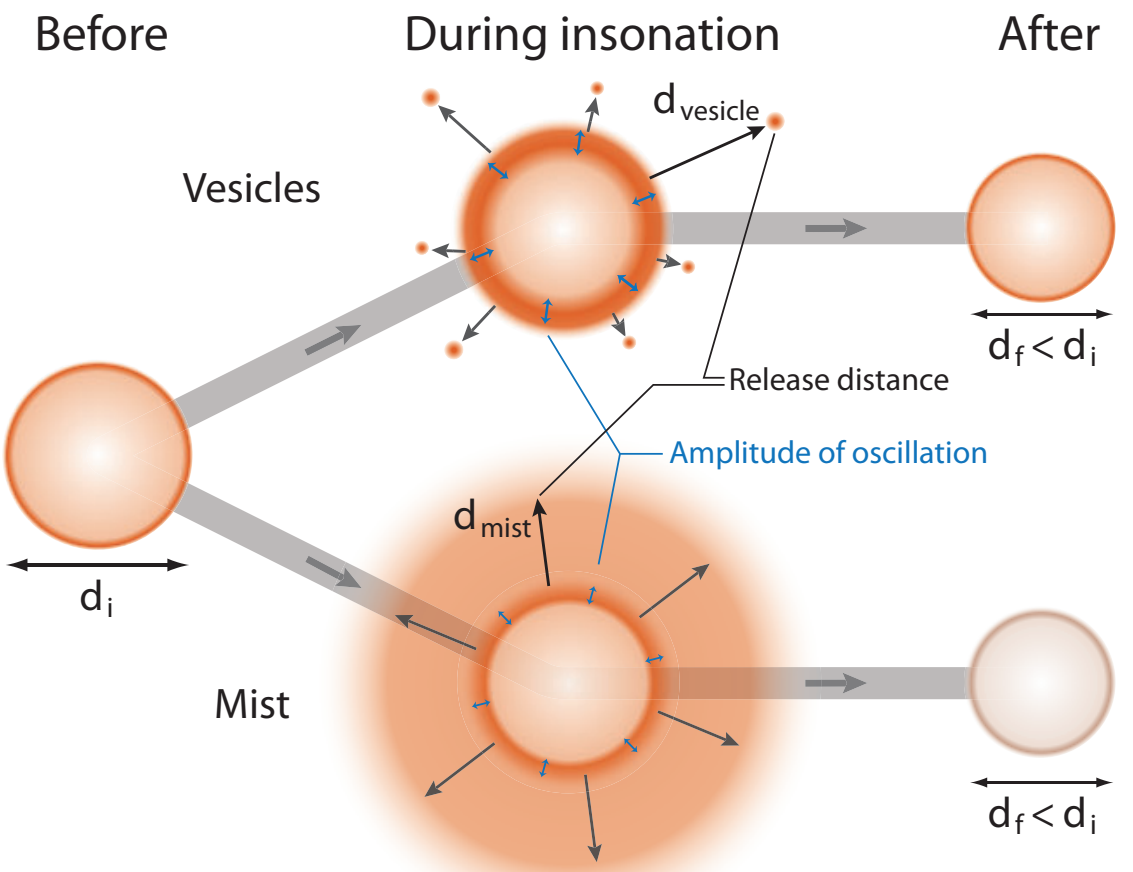

Figure 4.7: Schematic representation of the two observed types of release for both unloaded and loaded microbubbles.

\section{Vesicle release}

An example of the release of vesicles from a liposome-loaded bubble (sample $\mathrm{C}$ ) is shown in figure 4.8la. The bubble was insonified for 1000 cycles at a peak-negative pressure of $100 \mathrm{kPa}$. During acoustic activation of the bubble, small vesicles were shed from the bubble shell. Several vesicles were traced and their positions are plotted as a function of time in figure $4.8 \mathrm{~b}$ (inset shows the measured trajectories). The Stokes number (St) for a typical vesicle can be calculated to determine whether the vesicles will follow the flow pattern caused by acoustic streaming as a result of the bubble oscillations. The Stokes number is a dimensionless number that relates the response time of a spherical particle $\left(\tau_{p}\right)$ to the timescale of the flow field $\left(\tau_{f l}\right)$ :

$$
S t=\frac{\tau_{p}}{\tau_{f l}}
$$




\subsection{RESULTS}

where the response time is defined as:

$$
\tau_{p}=\frac{2 \rho_{p} R_{p}^{2}}{9 \mu} .
$$

Here $\rho_{p}$ is the mass density of the vesicle $\left(\sim \rho_{f l}\right), R_{p}$ is the typical vesicle radius $(\sim 500 \mathrm{~nm})$ and $\mu$ is the viscosity of the surrounding water. The typical timescale of the flow field is the period of the ultrasound excitation $(1 \mu \mathrm{s})$. This results in a Stokes number of 0.06 which is smaller than one, indicating that the vesicles will follow the bubble-induced flow field.

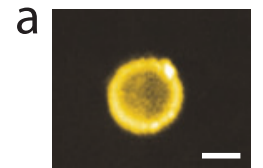

Before

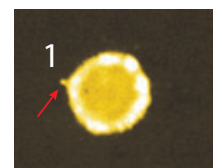

$\mathrm{t}=10 \mu \mathrm{s}$

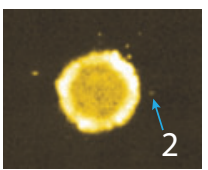

$20 \mu \mathrm{s}$

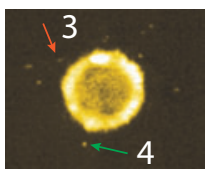

$30 \mu \mathrm{s}$

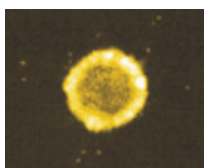

$40 \mu \mathrm{s}$

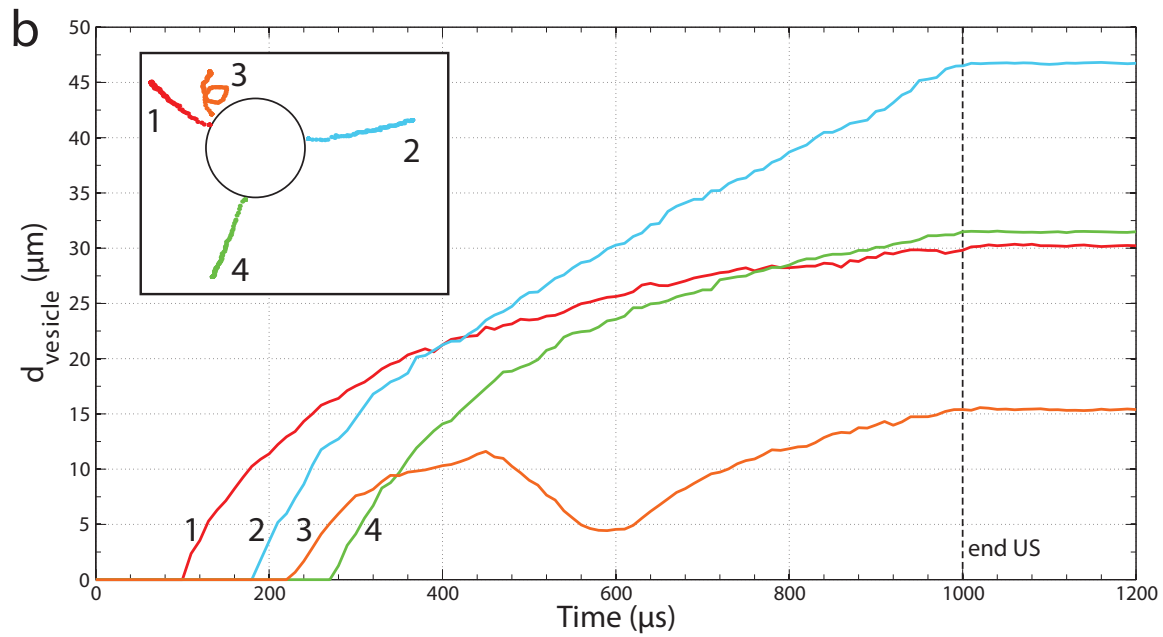

Figure 4.8: Release of vesicles from a liposome-loaded bubble (sample C, $100 \mathrm{kPa}$, 1000 cycles). a) Still frames from the high-speed fluorescence recording, before and during insonation, scale bar represents $10 \mu \mathrm{m}$. b) The distance of the vesicle with respect to their starting position on the bubble wall $\left(d_{\text {vesicle }}\right)$ over time of four vesicles. The inset shows the corresponding vesicle trajectories, obtained from the image data.

Marmottant et al. [75] showed that the oscillatory motion of the microbubble surface causes a time-averaged displacement of a particle close to the bubble, caused by the asymmetry introduced by the nearby wall. The high-speed recordings in this study only capture this time-averaged displacement, demonstrating a net repulsion of the vesicles (vesicle 1, 2, and 4 in figure 4.8). Vesicles that 

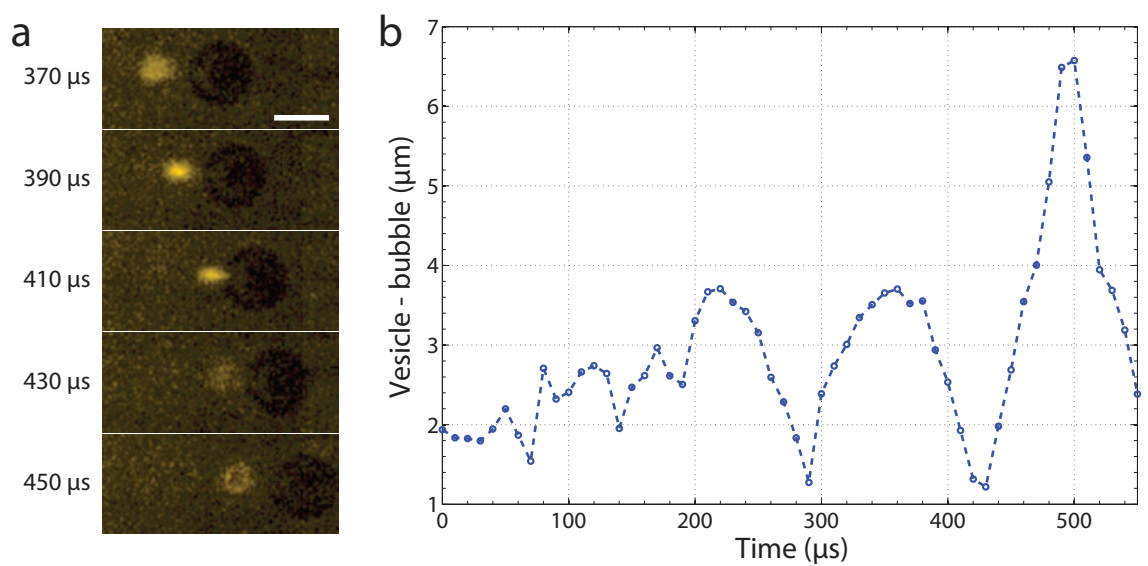

Figure 4.9: Looping motion of a vesicle caught in the wake of an oscillating microbubble (sample C, $60 \mathrm{kPa}, 1000$ cycles). a) Sample frames from a single loop, scale bar represents $5 \mu \mathrm{m}$. b) Vesicle center to bubble wall distance. Start of ultrasound at $t=0 \mu \mathrm{s}$

are close to the bubble surface (vesicle 3 in figure 4.8) can be trapped in one of the vortices of the flow field as has been predicted and shown experimentally by Marmottant et al. [76].

There is a large variation in the delay of the release for the different vesicles after the start of the insonation at $t=0 \mu \mathrm{s}$. The initial velocity with which the vesicles are released is on the order of $0.1 \mathrm{~m} / \mathrm{s}$. When the ultrasound is stopped at $\mathrm{t}=1000 \mu \mathrm{s}$, the vesicles immediately cease to move.

Another example of microfluidic vesicle transport is depicted in figure 4.9, which shows great similarity to the experiments performed by Marmottant and Hilgenfeldt [77], who reported on the deformation and lysis of lipid vesicles by a microbubble adherent to a solid substrate. The looping motion is clearly shown in the time evolution of the distance between the vesicle center and the bubble wall. Vesicle deformation can be seen in the image sequence, and is most pronounced at $\mathrm{t}=410 \mu \mathrm{s}$.

When viewed from the top, the flow field induced by the microbubble oscillations will be axisymmetric and when the bubble is not displaced during the oscillations, particles present in this field will only experience a force in the radial direction. It is known that bubbles in contact with a wall, excited at pressures on the order of $100 \mathrm{kPa}$ for long pulses, can display nonspherical oscillations due to parametric instabilities [3, 52, 78]. As a result, the symmetry of the flow field is broken. This is shown by the release and transport of vesicles from a liposome-loaded bubble, displaying shape oscillations, as depicted in figure 4.10 . 


\subsection{RESULTS}

As the bubble oscillates, the shape of the microbubble becomes nonspherical and from the image taken at $430 \mu \mathrm{s}$ after the start of the ultrasound the bubble shows 8 vertices, suggesting a mode $n=4$ shape oscillation [79]. At the vertices the bubble surface has the highest amplitude of oscillation, expelling particles into the surrounding liquid from these points, which matches the four lobes surrounding the bubble.

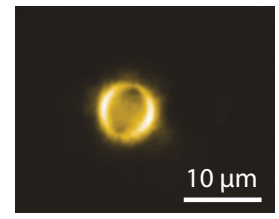

Before US

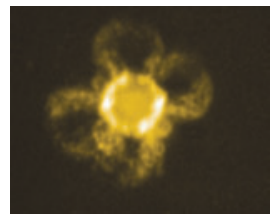

$230 \mu \mathrm{s}$

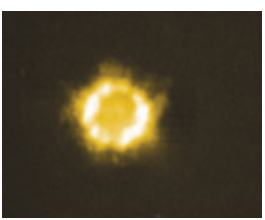

$\mathrm{t}=30 \mu \mathrm{s}$

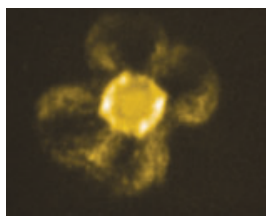

$330 \mu \mathrm{s}$

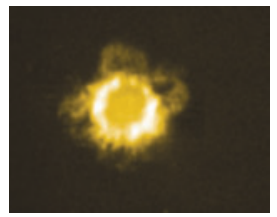

$80 \mu \mathrm{s}$

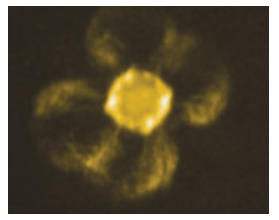

$430 \mu \mathrm{s}$

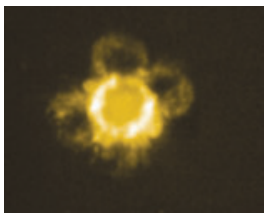

$130 \mu \mathrm{s}$

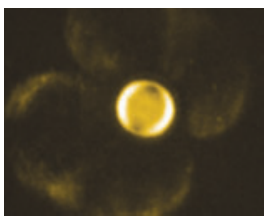

After US

Figure 4.10: Acoustic streaming from liposome-loaded bubble displaying shape oscillations. The microbubble was driven at a pressure of $80 \mathrm{kPa}$ for 1000 cycles.

\section{Mist release}

Figure 4.11 shows an example of the release of mist from an unloaded bubble (sample A). The mist is ejected instantaneously and at a velocity on the order of $1 \mathrm{~m} / \mathrm{s}$. Quickly after the onset of the ultrasonic activation, no fluorescence signal is noticeable from the rim of the bubble, suggesting that the bubble has lost a substantial part of its (fluorescent) shell material. This is confirmed by the shrinkage and subsequent dissolution of the bubble shown in the bright field images of figure $4.1 \mathrm{lb}$.

The front of the mist was traced and is shown in figure 4.11c. Again, after the ultrasound is stopped at $t=100 \mu$ s, the velocity of the mist is reduced to zero, suggesting that viscous effects dominate the transport of particles. The same analysis was performed for an unloaded bubble excited for 10 cycles and another one for 1000 cycles, also depicted in figure 4.11lc. Both curves show that the release takes place immediately after the start of the ultrasound. At a pulse duration of 10 cycles, the mist continues to spread, even after the ultrasound has stopped. Within the $10 \mu \mathrm{s}$ of actuation the mist particles did not spread 


\section{US-TRIGGERED RELEASE FROM LIPOSOME-LOADED BUBBLES}

far from the bubble surface. Marmottant and Hilgenfeldt [77] calculated that the flow field scales with $r^{-1}$ with $r$ being the distance to the bubble center. Particles close to the bubble surface will experience a stronger local flow field than far away from the bubble. At this short timescale and length scale, the advective forces are larger than the viscous drag forces.

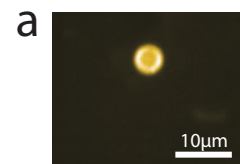

Before

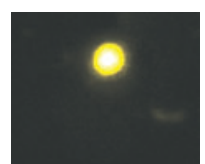

$\mathrm{t}=10 \mu \mathrm{s}$

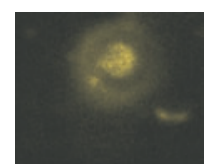

$20 \mu \mathrm{s}$

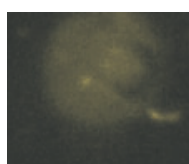

$30 \mu \mathrm{s}$

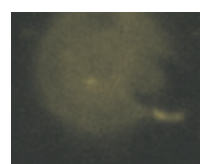

$40 \mu \mathrm{s}$
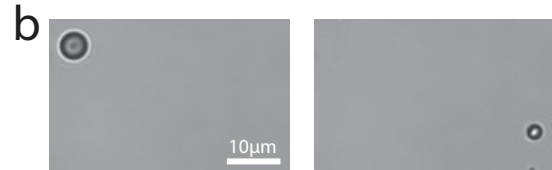

$\mathrm{t}=67 \mathrm{~ms}$

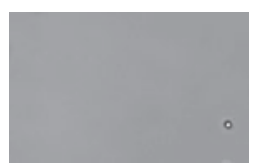

$134 \mathrm{~ms}$

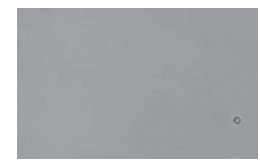

$200 \mathrm{~ms}$

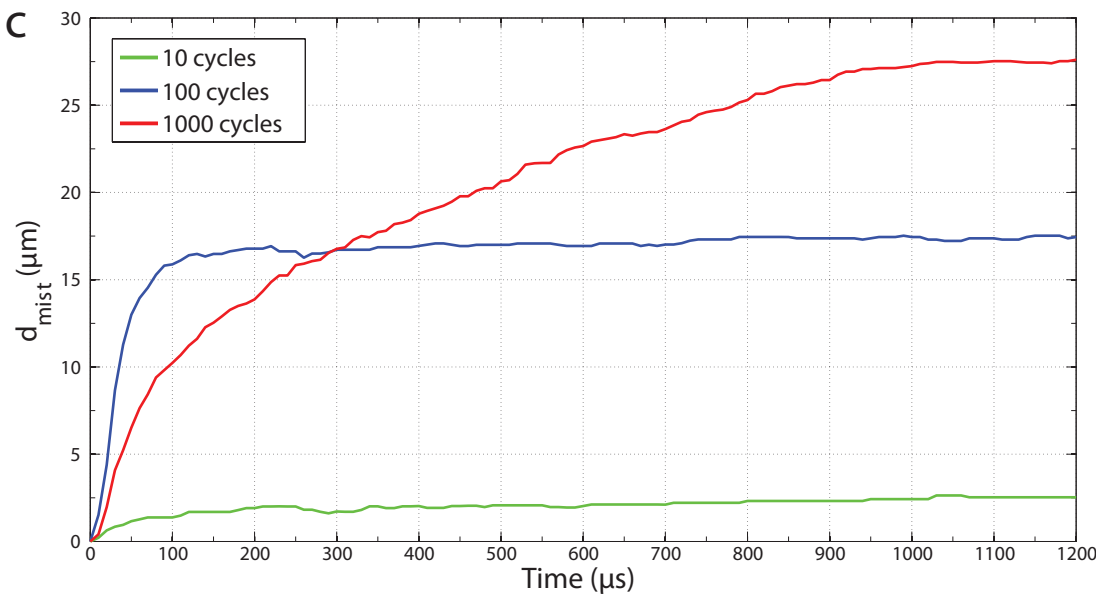

Figure 4.11: Release of a mist of particles from an unloaded bubble (sample $A$, $100 \mathrm{kPa}, 100$ cycles). a) Five consecutive frames from the high-speed fluorescence. b) Bright field images of the bubble before and after insonation. c) The distance of the front of the mist with respect to the initial position of the bubble wall $\left(d_{\text {mist }}\right)$ for three pulse lengths. The middle curve represents the bubble depicted in $a$ and $b$.

\section{Vesicles versus mist}

Both types of release were observed for unloaded and loaded microbubbles. Figure 4.12 shows the occurrence of vesicle release and mist release as a function of the ultrasound parameters. In general, it was observed that the threshold for 


\subsection{RESULTS}

release is lower for unloaded bubbles compared to loaded bubbles. For unloaded bubbles, insonation at $60 \mathrm{kPa}$ and 100 cycles causes nearly all microbubbles to release fluorescent shell material. For loaded bubbles a release of fluorescent material exceeding $75 \%$ was only found for a pressure of $100 \mathrm{kPa}$ and for 1000 cycles. In general, for higher pressures and longer pulses, the occurrence of release is increased.

The observation that release occurs more often for higher acoustic pressures indicates that the threshold of release depends on the amplitude of bubble oscillation. Luan et al. [73] have shown that the damping is increased substantially by loading a bubble with liposomes, due to an increase in the shell viscosity.

The low number of experiments where both vesicle release and mist release was observed, indicates that there may be a competition between the two types of release. The graphs in figure 4.12 show that the ratio of mist release versus vesicle release increases with higher pressures. Combined with the fact that mist release is already observed at a pulse duration of 10 cycles, this demonstrates that occurrence of mist release is mainly pressure dependent. On the other hand, the release of vesicles has a higher dependence on the pulse duration, as vesicle release already takes place at a pressure of $20 \mathrm{kPa}$, where no mist is observed.
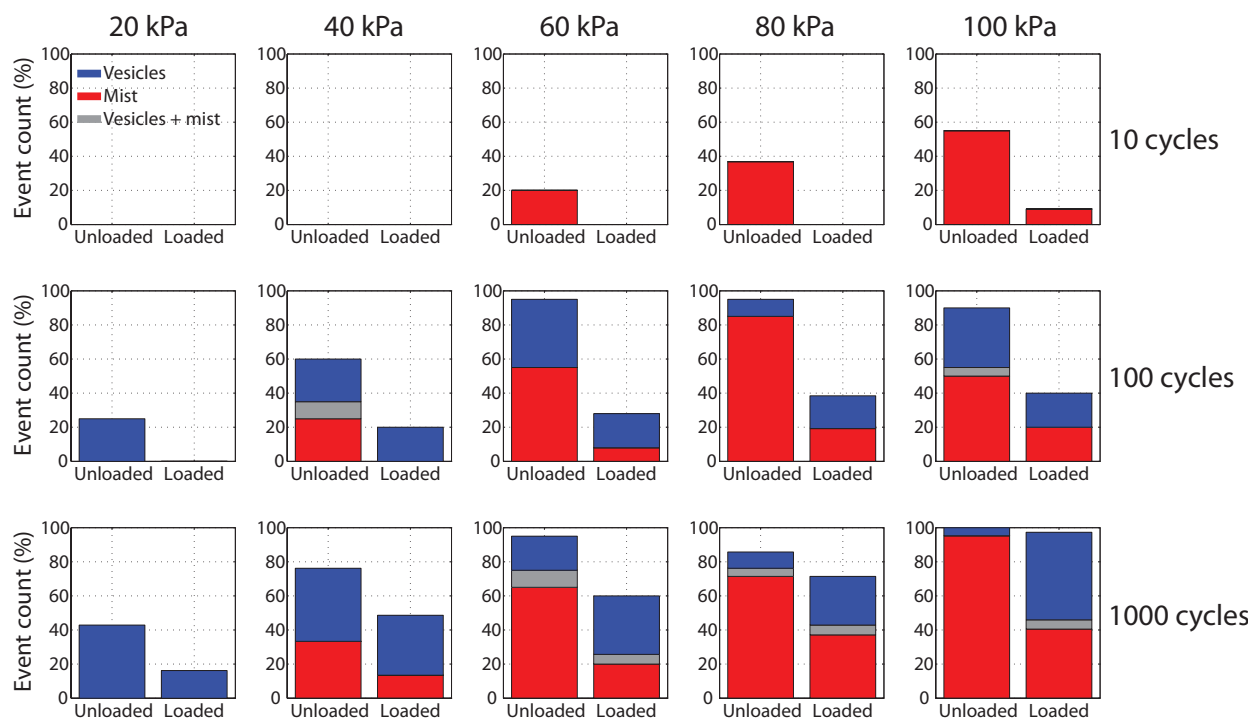

Figure 4.12: Occurrence of vesicle release and mist release for both unloaded and liposome-loaded microbubbles as a function of applied acoustic pressure and pulse duration. 


\subsubsection{Release distance}

To evaluate the length scale on which the release propagates, the maximum distance over which vesicles are transported during acoustic activation was determined for all microbubbles. Only the data from the liposome-loaded microbubbles is presented. For unloaded microbubbles, the measured distances were higher, as expected, but typically of the same order of magnitude. Figure 4.13 shows the release distance for liposome-loaded microbubbles as a function of the ultrasound parameters. The maximum release distance is approximately $15 \mu \mathrm{m}$, which is approximately 5 times the mean bubble radius. At higher pressures and for longer pulses, the vesicles are transported over a larger distance.

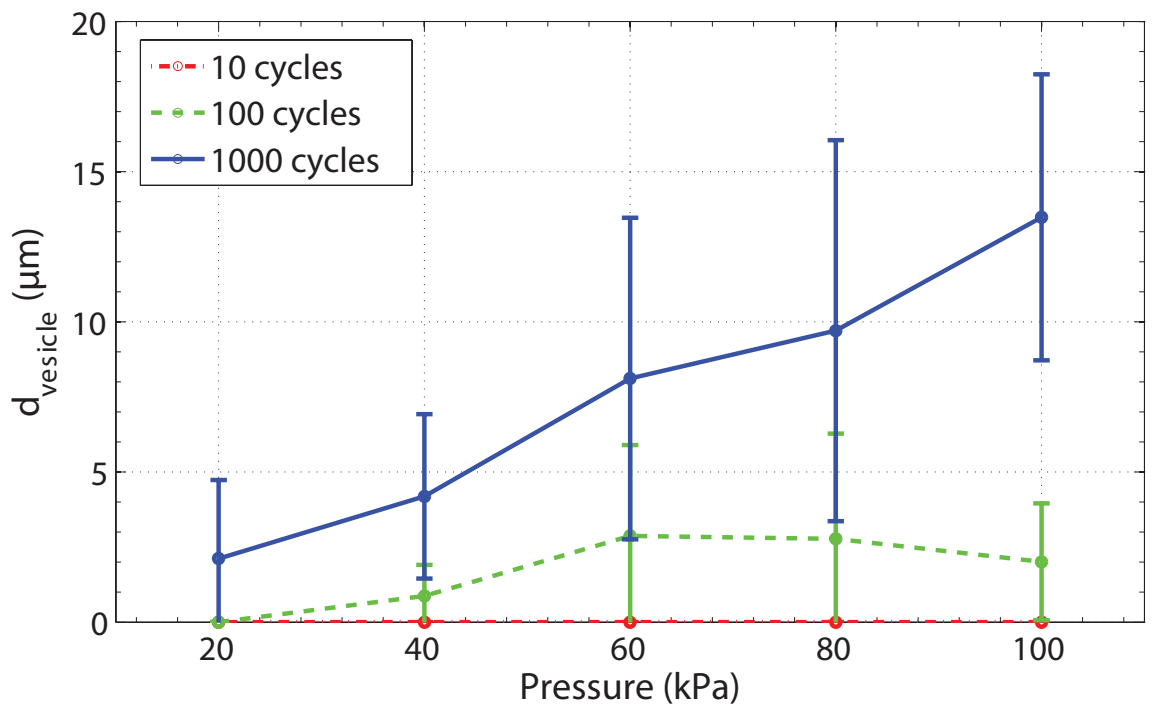

Figure 4.13: Release distance of vesicles from liposome-loaded microbubbles.

When fluorescent material is shed in the form of molecular or submicron particles, the distance over which the mist is spread, is increased up to a maximum of approximately $60 \mu \mathrm{m}$, as shown in figure 4.14 . The graph depicts the release distance for mist from liposome-loaded microbubbles for all ultrasound settings. Again, higher pressures and longer pulses result in an increased displacement of the released fluorescent material. 


\subsection{CONCLUSIONS AND DISCUSSION}

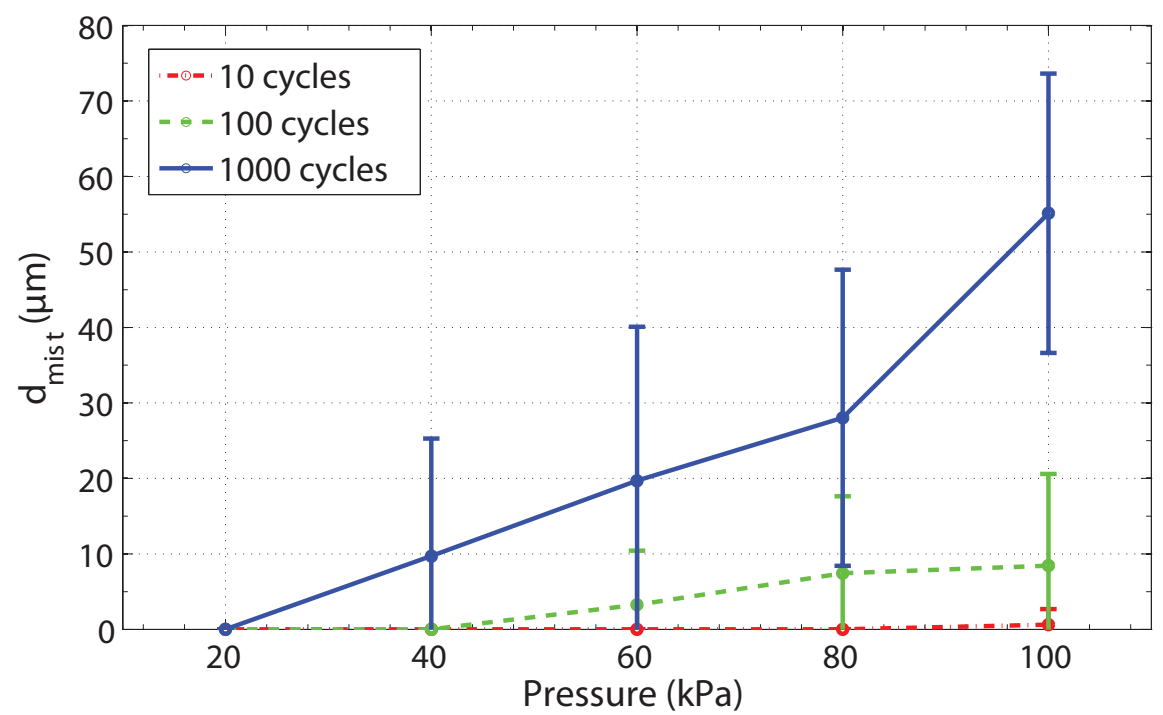

Figure 4.14: Release distance of mist from liposome-loaded microbubbles.

\subsection{Conclusions and discussion}

High-speed fluorescence imaging provides detailed, time-resolved information about the ultrasound-triggered release of phospholipids and liposomes from microbubbles. It gives valuable insight into the ultrasound parameters required for ultrasound-triggered drug delivery. Even below the threshold for release, an interesting phenomenon occurs, as the fluorescent shell material rearranges due to the microbubble oscillations. Rapid lateral displacement is observed of submicron structures that move around the bubble surface.

Two distinct types of release mechanisms were observed: the release of individually resolved vesicles with a size down to a micrometer and the release of molecular and submicron particles. Both mechanisms were observed for loaded and unloaded microbubbles, indicating that vesicles and mist can consist of phospholipids as well as liposomes. There appears to be a competition between the two release types, which remains unexplained for now.

The release of vesicles from the bubble surface takes place at a velocity on the order of $0.1 \mathrm{~m} / \mathrm{s}$. There can be a substantial delay between the onset of ultrasound and the release of vesicles, which is on the order of hundreds of microseconds. Therefore a pulse duration of 100 cycles is required to achieve vesicle release and an increase in the number of cycles leads to an increased release, whereas the applied pressure is of less influence for this type of release. 
At the highest pressure and for the longest pulse duration, the typical distance to which vesicles are released is five times the initial bubble radius.

The release of mist yields velocities on the order of $1 \mathrm{~m} / \mathrm{s}$ and occurs instantaneously at the onset of ultrasound. The radially expelled mist is already observed at a pressure of $40 \mathrm{kPa}$ and its occurrence is increased for increasing pressure. At the highest pressure and for the longest pulse duration, the mist can propagate over a distance of twenty times the initial bubble radius.

The unloaded bubbles clearly show a lower threshold for release, which can be a result of higher amplitudes of oscillation as compared to those of loaded bubbles at the same driving pressure. This effect can be attributed to a higher damping coefficient for liposome-loaded bubbles due to their increased shell viscosity.

When particles are expelled into the surrounding fluid the local flow field, caused by the microbubble oscillations, drives the displacement of the particles. These particles act as tracer particles, revealing the acoustic streaming patterns of the microbubble. When the bubble oscillations are stopped, the particles are slowed down within nanoseconds by the viscosity of the surrounding water, which is highly viscous at a length scale of microns. Consequently, at low acoustic pressures the release takes place at a length scale on the order of the size of the microbubble. For drug delivery applications a larger distance may be desirable to enhance the interaction area of the drug with the surrounding tissue. On the other hand, when microbubbles are close to the vascular endothelial cell layer, for instance as a result of targeting of the microbubbles, a localized release that is contained within the boundary layer of the blood flow could be beneficial. The local flow field and fluid properties in vivo, such as viscosity and the concentration of red blood cells, will influence the spreading and mixing of the released material.

To fully capture the mechanisms of release, fluorescence imaging on the timescale of the bubble oscillations is required in order to visualize the exact moment of release within a single ultrasound cycle. Furthermore, experiments have to be performed that can distinguish an intact liposome from a ruptured liposome, in order to evaluate not just the release of liposomes but also the subsequent release from liposomes, by shear flow, through ultrasound activation, or simply through a diffusive process. This could be achieved e.g. by filling the liposomes with a fluorescent label that is fully quenched inside the liposomes and only fluoresces once the concentration is reduced upon disruption of the liposome and release into the unsuspended liquid [10]. 
4.4 CONCLUSIONS AND DISCUSSION 


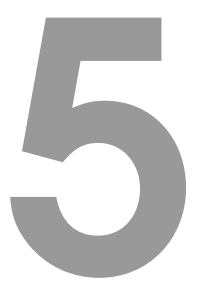

\section{Time-resolved high-speed fluorescence imaging of microbubble-induced sonoporation}

The uptake of propidium iodide (PI) by endothelial cells induced by microbubble oscillations was visualized at a millisecond timescale using high-speed fluorescence imaging. Single microbubbles were insonified at a driving frequency of $1 \mathrm{MHz}$ and at acoustic pressures varying from 200 to $1200 \mathrm{kPa}$ for a duration of 10 and 100 cycles. At a pressure of $200 \mathrm{kPa}$ and $10 \mathrm{cycles}, 50 \%$ of the cells showed uptake of $\mathrm{Pl}$, and this percentage increased to $90 \%$ for a pressure of $400 \mathrm{kPa}$. At a pressure of $1200 \mathrm{kPa}$ all cells showed uptake of PI. The high-speed fluorescence recordings revealed that a localized pore in the cell membrane is formed at the position of the bubble. Uptake was observed within several milliseconds after insonation and the size of the induced pore was found to be dependent on the bubble radius. Furthermore, the inflow of $P I$ is diffusion-driven. The pore is formed temporarily and closes within several seconds after the ultrasound exposure. 


\subsection{INTRODUCTION}

\subsection{Introduction}

Gas-filled coated microbubbles have been used as ultrasound contrast agents (UCAs) for over thirty years. When injected into the blood stream they scatter ultrasound very effectively due to the compressibility of the gas core, providing an enhanced signal from the blood pool. This can be used in diagnostic ultrasound imaging to improve the blood-to-tissue contrast in organ perfusion imaging [1, 54].

Since several years, microbubbles are investigated for their therapeutic capacity [58]. Microbubbles can be loaded with a therapeutic agent, for instance by attaching drug-loaded liposomes to the surface of the bubbles [8]. At low acoustic pressures, the bubbles respond to the ultrasound while the drugs remain inside the bubble or attached to the bubble surface. A burst of ultrasound at an elevated pressure causes large oscillations or cavitation of the bubble triggering the release of the drug payload. Another method is based on co-administration of microbubbles and a therapeutic agent [?].

In both cases microbubbles are known to play a key role in the process of locally enhanced delivery of the therapeutic agent into the surrounding tissue. This technique, called sonoporation, has been widely studied in order to reveal the underlying mechanism and quantify the required ultrasound parameters [24, 80-87]. It has been shown that bubble oscillations cause a transient increase in cell membrane permeability, and this is often visualized by the uptake of nucleic acid binding fluorochromes such as SYTOX Green, YO-PRO, TO-PRO-3 and propidium iodide (PI) [88, 89]. Although the size of PI is on the order of a nanometer, it is impermeable to the cell membrane. Only when the cell membrane integrity is compromised, PI will cross the membrane and fluorescently label the RNA in the cell cytoplasm and the DNA in the cell nucleus.

To quantify the uptake of $\mathrm{PI}$, many reported studies insonify a large number of cells in the presence of a solution with a known concentration of contrast agent microbubbles. The cells are subsequently analyzed with a flow cytometer to determine the effectiveness of the sonoporation [80, 81, 86, 90-92]. This method, however, only provides statistical information about the process and no time-resolved information, let alone on individual bubble-cell interaction.

Several recent studies have shown fluorescence microscopy of single cell sonoporation which offers an improved temporal and spatial resolution compared to flow cytometry [24, 84, 93, 94]. The highest temporal resolution reported, was demonstrated in a recent study by Kooiman et al. [84] in which sonoporation was induced by applying 6 ultrasound pulses, each with a duration of 10 cycles. Already 80 milliseconds after insonation the fluorescence signal of bound PI was 
clearly visible, indicating that the sonoporation process had taken place at an even shorter timescale. The results of Van Wamel et al. [24] showed that the $\mathrm{PI}$ in the cell was concentrated near the contact area of the bubble and the cell.

A further enhanced temporal resolution can be achieved by measuring the transmembrane current (TMC) of a cell by applying a voltage clamp [82, 87, 95] . In theory, a sample rate of several kilohertz can be obtained. The TMC is directly related to the porosity of the cell membrane and can therefore serve as a measure of sonoporation. Results from Pan et al. [87] showed a distinct drop in the TMC during insonation of microbubbles in the proximity of a Xenopus oocyte, a popular model for gene and protein expression studies, with a temporal resolution in the order of milliseconds. However, they do not offer any insight about the location of the pores with respect to the position of the microbubble. Zhou et al. [82] also measured a decrease in the TMC during ultrasound excitation of microbubbles and oocytes and were even able to relate the change in TMC to the pore size, which was found to be in the order of $100 \mathrm{~nm}$. Again however, the interaction between the microbubbles and cells that led to the generated pores was not recorded. Furthermore, is was assumed that the measured cells only had one single pore, originating from the oscillations of a single microbubble. However, this was not supported by physical evidence.

Aside from TMC measurements, other techniques are used to judge the pore size after bubble-induced sonoporation or sonolysis, such as scanning electron microscopy (SEM) [92, 96, 97] and atomic force microscopy (AFM) [83, 85, 98] of which only Prentice et al. [83] were able to link the induced pore to an observed microbubble cavitation event. The estimated pore sizes were typically in the order of 50 to $500 \mathrm{~nm}$, in some cases confirmed by confocal microscopy of the uptake of large dextran-coupled fluorophores [92, 97], up to $16 \mu \mathrm{m}$ [83] and were obtained several seconds to minutes after the sonoporation event itself. Results from Zhou et al. [82] and Van Wamel et al. [24] showed that the pores can reseal on a timescale of seconds, which creates a large uncertainty for pore sizes obtained using AFM or SEM.

Visualizing the uptake of a model drug such as PI both at a relevant temporal resolution and spatial resolution is crucial to elucidate the physical mechanisms responsible for the sonoporation mechanism and bubble-induced cell membrane permeabilization. In this study, we investigate the relevant time scales at which the cell membrane permeabilization occurs. For this purpose the influx of PI into human umbilical vein endothelial cells (HUVECs) triggered by oscillations of phospholipid-coated microbubbles was studied at timescales ranging from a millisecond to a minute using combined high-speed fluorescence imaging at $1000 \mathrm{fps}$ and low-speed fluorescence imaging at $10 \mathrm{fps}$. 


\subsection{Materials and methods}

\subsubsection{Experimental setup}

\section{Cell culture}

HUVECs were isolated from an umbilical vein, by the method of Jaffe et al. [99], using trypsin solution (0.05\% trypsin/0.02\% EDTA in PBS; Invitrogen, United Kingdom). The obtained endothelial cells were cultured on $2 \mathrm{mg} / \mathrm{ml}$ fibronectin (Sanquin, The Netherlands) coated culture flasks. Culture medium consisted of endothelial basal medium (EBM-2, Lonza, Switzerland) supplemented with the EGM-2 SingleQuot kit (Lonza) to form EGM-2 medium. Cell cultures were maintained in a humidified atmosphere at $37^{\circ} \mathrm{C}$ and $5 \% \mathrm{CO}_{2}$. Medium was refreshed every 3-4 days. Confluent cultures were subcultured for up to 6 passages with the use of trypsin solution. For sonoporation experiments, HUVECs were cultured in an Opticell ${ }^{\mathrm{TM}}$ (Nunc, Thermo Scientific, Denmark) until confluence. At the start of the experiment, HUVECs were washed once with PBS after which EGM-2 medium was added containing $10 \mu \mathrm{g} / \mathrm{mL}$ propidium iodide (Sigma-Aldrich, The Netherlands) and approximately $1 \times 10^{5}$ microbubbles. For the laser damage threshold experiments, HUVECs were cultured in a microchannel ( $\mu$-Slide III, iBidi ${ }^{\circledR}$, Germany) until confluence. Before the experiment, the cells were washed once with PBS and were kept in PBS during the experiment.

\section{Microbubbles}

Phospholipid-coated microbubbles with a $\mathrm{C}_{4} \mathrm{~F}_{10}$ gas core were produced inhouse following a method described by Klibanov et al. [34]. The coating was composed of DSPC (59.4 mol\%; Sigma-Aldrich); PEG-40 stearate (35.7 mol\%; Sigma-Aldrich) and DSPE-PEG(2000) (4.9 mol\%; Avanti Polar Lipids, USA).

$100 \mu \mathrm{L}$ of the concentrated microbubble solution was diluted and washed once with demineralized water. Next, the sample was diluted again in demineralized water to achieve a concentration of $1 \times 10^{6}$ microbubbles $/ \mathrm{mL}$, of which $100 \mu \mathrm{L}$ was added to the EGM-2 medium. This concentration was chosen to obtain a bubble-to-cell ratio of less than one, in order to study the interaction between a single bubble and a single cell.

\section{High-speed fluorescence imaging of PI uptake}

After addition of the medium containing $\mathrm{PI}$ and microbubbles, the Opticell ${ }^{\text {TM }}$ was placed in a water bath, kept at $37^{\circ} \mathrm{C}$, located underneath an upright fluorescence microscope setup, depicted in figure 5. . la. The microscope was equipped with a $60 \times$ water-immersion objective ( $N A=0.9$; Olympus, The Netherlands). 
To achieve sufficient fluorescence signal at a millisecond timescale, a continuous wave laser (5 W@532 nm; Cohlibri; Lightline, Germany) was employed for fluorescence excitation of the PI. The laser light was gated using an acoustooptic modulator (AOM) (AOTF.nC-VIS; AA Optoelectronic, France) to generate pulses with a duration of $60 \mu \mathrm{s}$ to reduce heating of the sample and to minimize photobleaching of the fluorochrome. In principle, fluorescence imaging of $\mathrm{PI}$ up to a frame rate of $25 \mathrm{Mfps}$ can be performed, using this laser. However, since the uptake of PI takes place at a timescale of milliseconds instead of microseconds, these high frame rates are not necessary to fully capture the dynamics of the process.
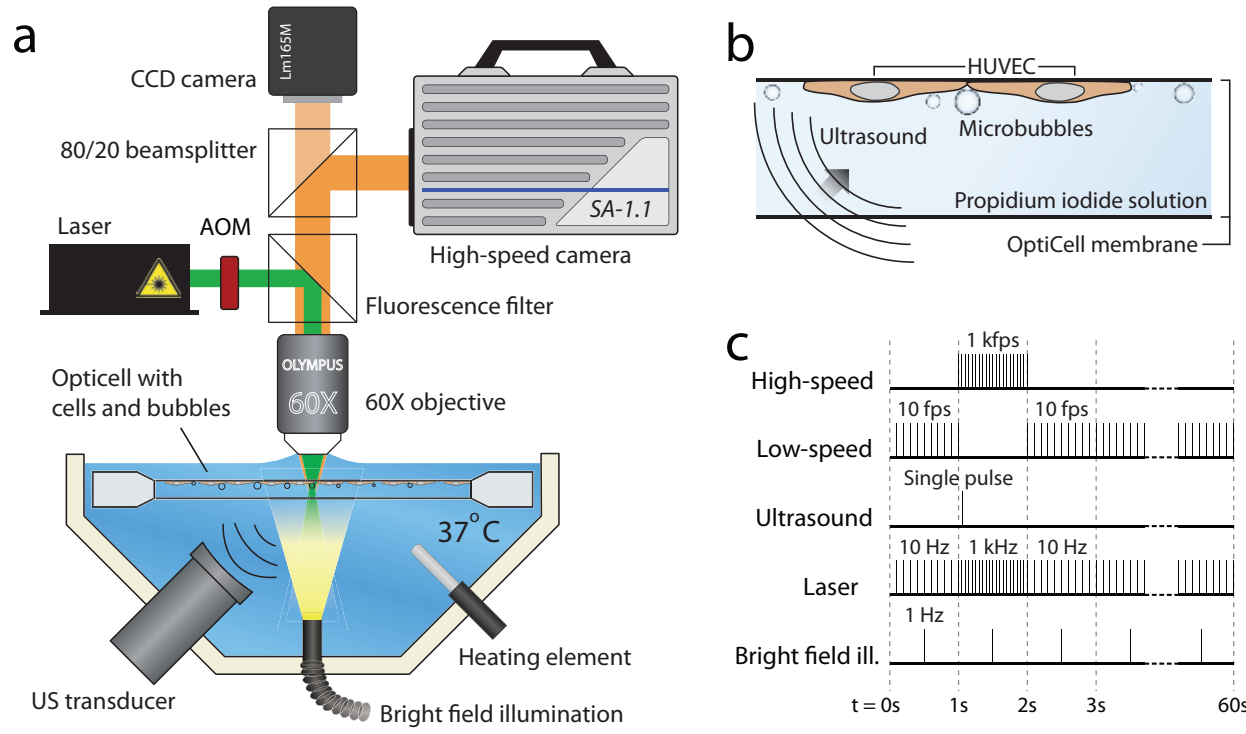

Figure 5.1: a) Schematic representation of the experimental setup. The Opticell ${ }^{\mathrm{TM}}$ containing HUVECs in a PI-solution with microbubbles is placed in a water bath, located underneath a fluorescence microscope setup with a high-speed camera operating at $1000 \mathrm{fps}$ and a CCD-camera for low-speed recordings. b) Close-up of the Opticell ${ }^{\mathrm{m}}$. c) Timing diagram of the experiment.

The microbubbles and cells were insonified by single, sine-wave ultrasound pulses with a duration of 10 or 100 cycles at a frequency of $1.0 \mathrm{MHz}$ and at three peak-negative pressures $(200,400$ and $1200 \mathrm{kPa})$ using a focused, single element transducer (C302; Panametrics, USA). The pressures generated by the transducer were calibrated with a $2 \mathrm{~mm}$ PVDF needle hydrophone (Precision Acoustics, UK). The ultrasound was triggered such that the pulse ended several microseconds prior to the laser pulse. 


\subsection{MATERIALS AND METHODS}

High-speed fluorescence recordings of the inflow of PI were made with a CMOS-based high-speed camera (SA-1.1; Photron Ltd,UK), operating at a frame rate of $1000 \mathrm{fps}$, starting $10 \mathrm{~ms}$ prior to ultrasound excitation for a duration of 1 second. The microscope was fitted with a 80/20 beamsplitter to allow for simultaneous low-speed fluorescence recordings at $10 \mathrm{fps}$, using a CCDbased camera (LM165M; Lumenera Corporation, Canada). Low-speed recordings lasted until the cell was completely stained with PI.

Pulsed bright field illumination was produced by a shuttered halogen light source (KL1500LCD, Schott, Germany) at a repetition frequency of $1 \mathrm{~Hz}$, to monitor the cell position and morphology throughout the experiment. A timing diagram is shown in figure $5.1 \mathrm{c}$. The repetition rate of the AOM was varied to match the frame rate of the high-speed and low-speed recordings. This resulted in a duty cycle of the laser illumination of $6 \%$ during the high-speed recordings and $0.06 \%$ during the low-speed recordings.

For each sonoporation experiment the high-speed and low-speed fluorescence recording were analyzed using Matlab. The area of the cell that was stained with PI was measured for all frames to capture the dynamics of the inflow of PI. A region of interest containing a single cell was selected. The fluorescence image recorded several microseconds after the ultrasound excitation was taken as a reference background and extracted from all subsequent images. The position and size of the microbubble was determined in order to exclude the area occupied by the bubble from the analysis to avoid a bias signal due to possible shrinkage of the bubble [33]. The $1 \mathrm{kHz}$ repetition rate illumination of the laser caused photobleaching during the high-speed recording, for which the images were corrected. This was done by monitoring the background fluorescence signal emitted by the EGM-2 medium at various distances from the center of the laser spot over the course of the high-speed recording. Since the intensity of the laser spot was known, a time-dependent correction coefficient for every position in the fluorescence image could be calculated.

\section{Laser damage threshold}

To rule out the influence of the laser illumination on the cultured cells during the sonoporation experiments, the damage threshold of the endothelial cells was investigated. HUVECs were exposed to high-intensity laser irradiation for laser pulse lengths varying from $100 \mu \mathrm{s}$ to $1 \mathrm{~s}$. The spatial distribution of a laser pulse had a gaussian profile with a maximum laser intensity of $10 \mathrm{MW} / \mathrm{cm}^{2}$ and a width of $30 \mu \mathrm{m}$ at $1 \mathrm{MW} / \mathrm{cm}^{2}$. Regions of $1 \times 1 \mathrm{~mm}$ were marked in the microchannel and laser pulses were delivered spaced $50 \mu \mathrm{m}$ apart, following a rectangular pattern. It can be calculated that $70 \%$ of the area was 
exposed to a laser intensity higher than the maximum intensity applied during the sonoporation experiments.

In a separate microchannel, which served as a positive control, cells were incubated with $3 \mathrm{nM}$ tumor necrosis factor-alpha (TNF- $\alpha$ ) and $50 \mu \mathrm{M}$ cycloheximide to induce cell death. 24 Hours after exposure, the viability of the cells was tested by adding PI to the cells.

\subsubsection{Numerical model}

The fluorescence recordings provide a measure for the concentration of $\mathrm{PI}$ inside a cell as a function of both time and space. When we assume that the inflow of $\mathrm{PI}$ is localized to a single pore and that the process is diffusion-driven, we can derive information about the pore size and pore opening time by comparing the experimental results to a physical model.

Physically, an endothelial cell is complex to model: it has an irregularly shaped three-dimensional volume with complex viscous liquid properties and a concentration of RNA and DNA that are not constant throughout the cell. Furthermore, these properties can also vary over time. The model that is presented here is therefore a simplified representation of the processes that take place during a sonoporation event and is only used as a qualitative description. As a first attempt, the model was based on the one-dimensional diffusion equation:

$$
\frac{\partial c}{\partial t}=D \frac{\partial^{2} c}{\partial x^{2}}
$$

where $c(x, t)$ is the concentration of $\mathrm{PI}$ and $D$ is the diffusion constant of $\mathrm{PI}$ inside the cell, and $x$ and $t$ are the space and time variables. The diffusion equation is discretized using a centered second difference scheme in space and forward difference scheme in time:

$$
\frac{c(i, t+\Delta t)-c(i, t)}{\Delta t}=D \frac{c(i+1, t)-2 c(i, t)+c(i-1, t)}{(\Delta x)^{2}} .
$$

The model is schematically depicted in figure 5.2 with on the left hand side the bubble-induced pore, through which the PI diffuses into the cell. The concentration of PI outside the cell is assumed to be constant with a value of $c_{0}$ and at the far end of the cell $(x=1)$ a Dirichlet boundary condition is applied. The pore is opened at $t=0$ and when the pore is closed after a certain time $t_{\text {close }}$, this boundary condition also applies to the left hand side of the cell 
$(x=0)$ :

$$
\begin{array}{ll}
c(0, t)=c(1, t)=c_{0} & x=0,0<t \leq t_{\text {close }} \\
c(0, t)=c(1, t) & x=0, t>t_{\text {close }} \\
c(N, t)=c(N-1, t) & x=1, t>0 .
\end{array}
$$

Figure $52 \mathrm{~b}$ and $\mathrm{c}$ depict the time evolution of the PI concentration for $t_{\text {close }}=\infty$ and $t_{\text {close }}=5 \mathrm{~s}$. When the pore does not close, the concentration of $\mathrm{PI}$ will increase until equilibrium is reached. When the pore is closed the concentration near the pore decreases until equilibrium inside the cell is obtained. The detectable concentration of $\mathrm{PI}$ is much lower than the outside concentration. It will therefore take much shorter to completely stain the cell than to reach an equilibrium for the concentration of PI.

The model predicts the time evolution of the concentration of PI throughout the cell. This can be related to the measured fluorescence signal by assuming that the diffusivity of PI in the cytoplasm and nucleus is constant. The recordings show that the PI spreads homogeneously throughout the cell without any variations in velocity, even at the border of the nucleus, which validates this assumption.

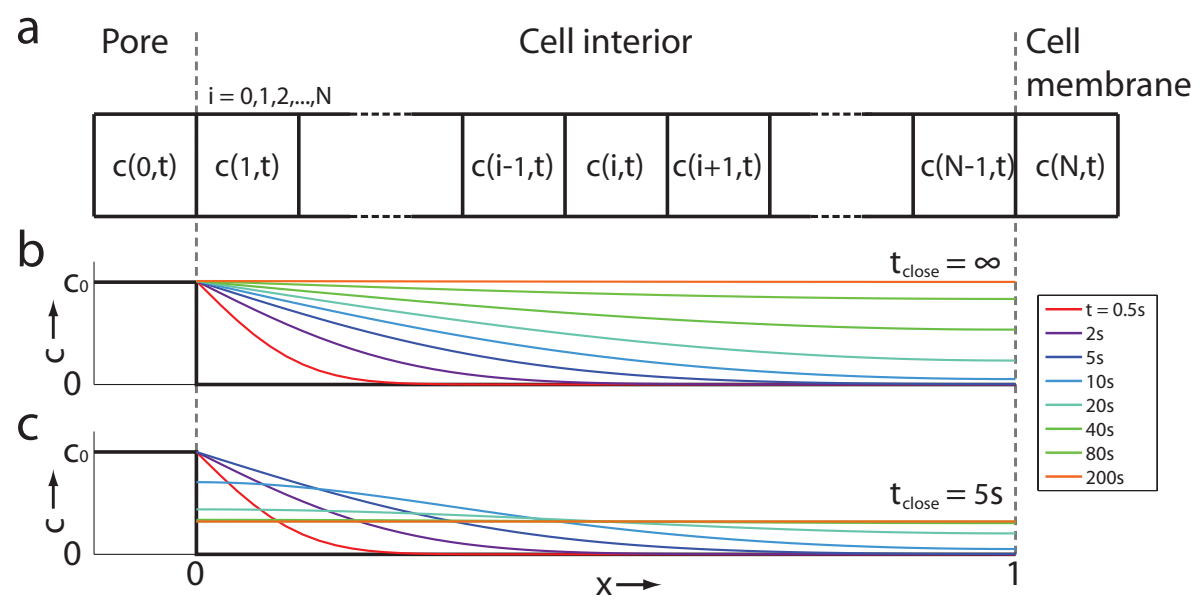

Figure 5.2: One-dimensional numerical model of the PI uptake by an endothelial cell. a) Numerical grid. b) Time evolution of the PI concentration for $t_{\text {close }}=\infty$ (black line indicates initial condition) and c) for $t_{\text {close }}=5 \mathrm{~s}$.

Furthermore, it is assumed that the fluorescence signal is proportional to the local $\mathrm{PI}$ concentration. Each cell contains approximately $6.6 \cdot 10^{9}$ base pairs. Since one $\mathrm{PI}$ molecule binds per $4-5$ base pairs, $1.5 \cdot 10^{9}$ binding sites 
are available. The cultured endothelial cells have a cell volume of approximately $5 \cdot 10^{-15} \mathrm{~L}(3 \times 40 \times 40 \mu \mathrm{m})$, leading to a binding site concentration of $3 \cdot 10^{23} / \mathrm{L}$. The PI concentration in the surrounding medium of the cell was $10 \mu \mathrm{g} / \mathrm{L}$, which gives rise to a concentration of dye molecules of $1 \cdot 10^{22} / \mathrm{L}$, considering the molecular weight of $668 \mathrm{Da}$. When the $\mathrm{PI}$ enters the cell and spreads throughout the cytoplasm and nucleus, the number of binding sites is abundant. Since the binding of PI to RNA or DNA is assumed to take place at a timescale several orders of magnitude shorter than a millisecond, the fluorescence signal from $\mathrm{PI}$ can be considered to be proportional to the concentration of PI.

From the model several quantities can be obtained, such as the area that is stained by PI, the total fluorescence intensity and the fluorescence intensity at a given point in the cell.

\subsection{Results}

\subsubsection{Laser damage threshold}

Figure $[5.3$ shows two exposed regions and a negative and positive control area. No difference in cell viability is noticeable between the exposed area and the negative control. The positive control region shows a larger number of stained cells and many cells detached due to the induction of cell death.

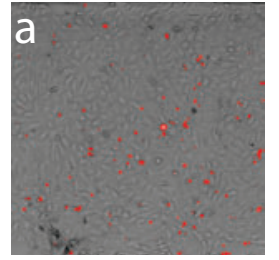

Pulse length $=10 \mathrm{~ms}$

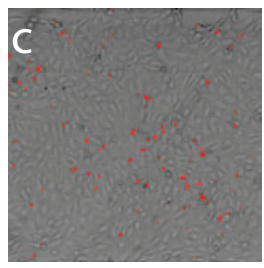

Negative control

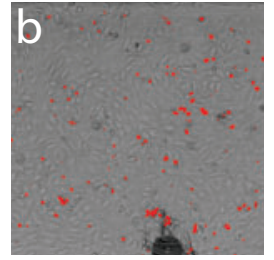

Pulse length $=1 \mathrm{~s}$

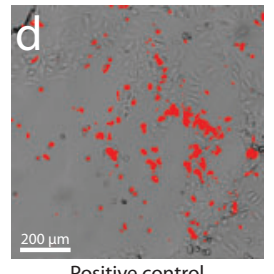

Positive control

Figure 5.3: Fluorescence $(\mathrm{PI})$ and bright field overlays of HUVECs cultured in a microchannel. The cells in a) and b) were exposed to high-intensity laser irradiation. Per image 500 evenly distributed laser pulses were applied. The positive control cells (d) were incubated with TNF- $\alpha$. 


\subsection{RESULTS}

The results of cell counting, shown in figure 15.4, confirm that the cultured HUVECs are not affected by the laser illumination, even at a pulse length of 1 second. Since the duty cycle during the sonoporation experiments is $6 \%$ and only for the high-speed recording, we can safely neglect the influence of the laser on cell viability.

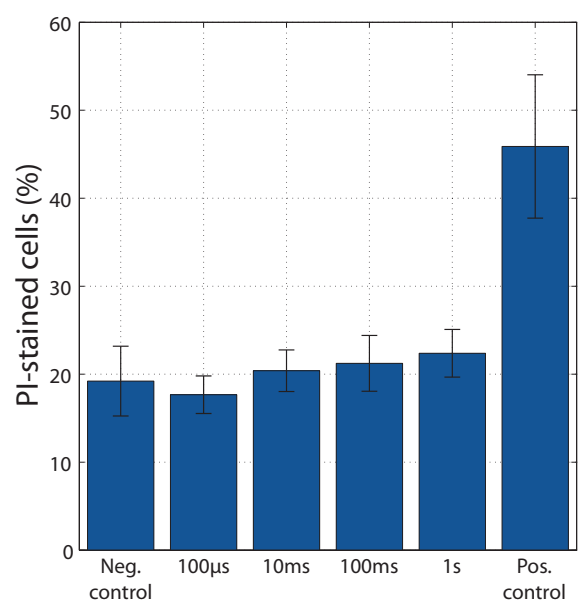

Figure 5.4: Analysis results of the four areas depicted in figure 5.3. No difference in cell viability is noticeable between the negative control and the exposed regions.

\subsubsection{Experiment statistics}

A total number of 104 sonoporation experiments were performed on single cells in contact with a single microbubble. This number includes 8 control experiments without ultrasound, where a microbubble was present and laser irradiation was applied, and 10 control experiments without a microbubble, where ultrasound ( $1200 \mathrm{kPa}, 100 \mathrm{cycles})$ and laser irradiation were both applied. An overview of the result of these 104 experiments is given in figure 5.5, indicating that no cells were porated during the control experiments.

Figure 5.5 shows that at a peak-negative pressure of $200 \mathrm{kPa}, 50 \%$ of the cells showed uptake of PI by applying 10 cycles of ultrasound and $64 \%$ showed uptake after 100 cycles. With increasing ultrasound pressure, the number of sonoporation events increased as well: at $400 \mathrm{kPa}$, approximately $90 \%$ of the cells were porated and at $1200 \mathrm{kPa}$ all cells showed uptake of $\mathrm{PI}$ following ultrasound excitation, regardless of the pulse length of the ultrasound. Within less than two minutes after insonation, all porated cells displayed fluorescence coming from the complete area of the cell, including the cell nucleus. 


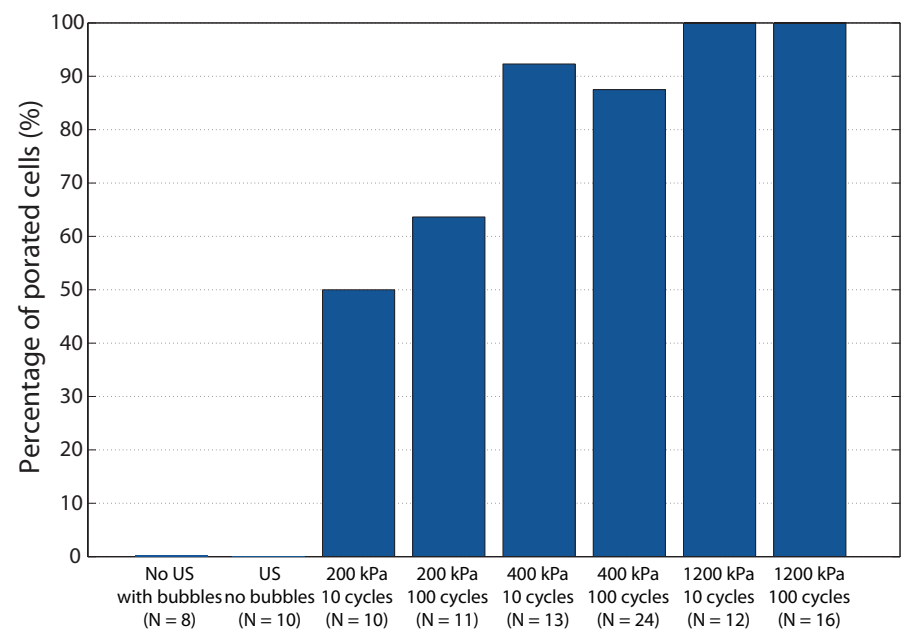

Figure 5.5: Percentage of porated cells as a function of the applied peak-negative pressure. A total number of 104 experiments was carried out on single cells in contact with a single microbubble.

\subsubsection{High-speed fluorescence imaging of PI uptake}

Figure 5.6 shows an example of a typical experiment. Several still frames from the high-speed and low-speed fluorescence recording are depicted in figure 5.60 and $b$. The corresponding area-time curve is given in figure $5.6 \mathrm{c}$. A microbubble with a radius of $2.4 \mu \mathrm{m}$ located against the cell membrane was insonified at $200 \mathrm{kPa}$ for 10 cycles. The inset in figure $5.6 \mathrm{c}$ shows that immediately after insonation the fluorescence signal from PI binding to RNA in the cell cytoplasm is visible. Since the bubble image was extracted from the analysis window, the first data points show only a very small increase in the area. After $25 \mathrm{~ms}$, a circular stain has become clearly visible and that area steadily grows over the course of the high-speed recording. Within 16 seconds after ultrasound excitation, a fluorescence signal is present in the complete cell cytoplasm and cell nucleus. The overall shape of the area-time curve suggests that the inflow of $\mathrm{PI}$ into the cell is a diffusion-driven process.

Despite correcting the high-speed image data for photobleaching a clear discontinuity in the graph of figure 5.6 is visible at $t=1 \mathrm{~s}$. It is unclear what the exact cause for this discontinuity is, however a possible explanation is a difference in the photobleaching rate constant of PI inside the cell and the surrounding medium. The growth rate of the stained area recovers when the pulse repetition rate of the laser is decreased back to $10 \mathrm{~Hz}$ for the low-speed recording. 


\subsection{RESULTS}

\section{a}
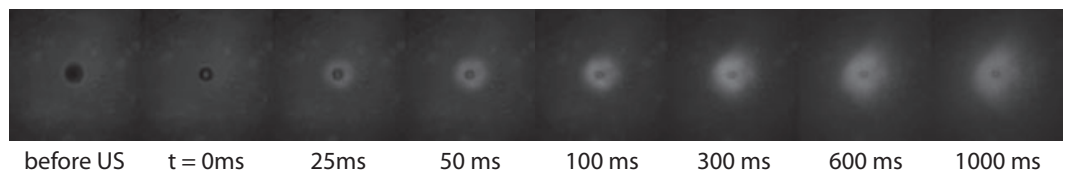

b
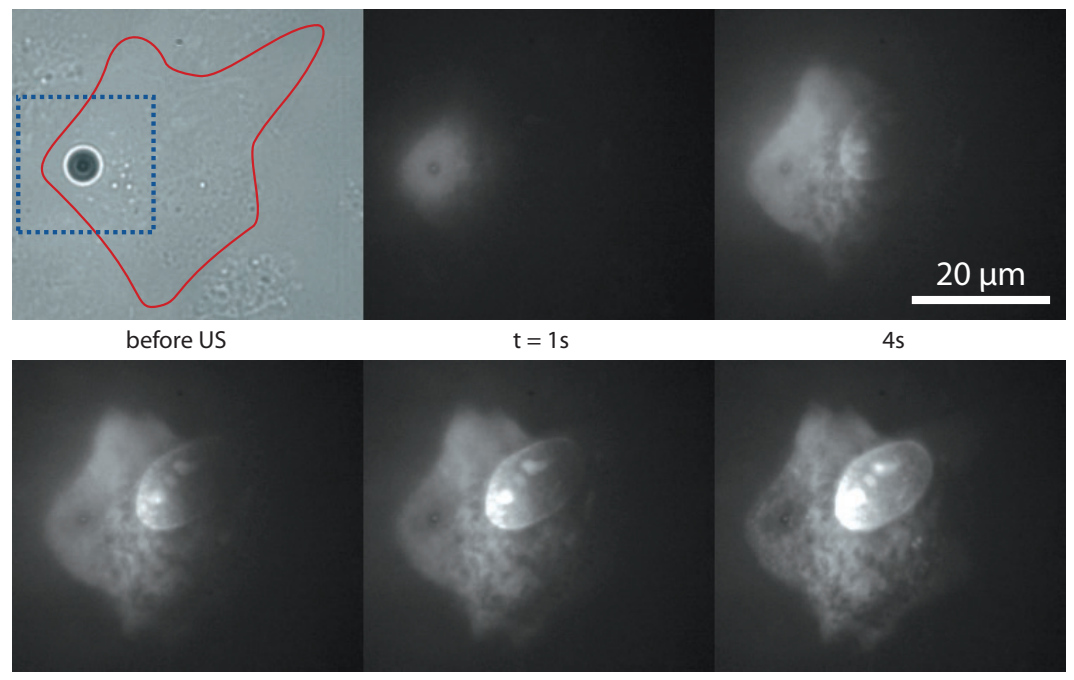

$7 \mathrm{~s}$

$10 \mathrm{~s} \quad 16 \mathrm{~s}$

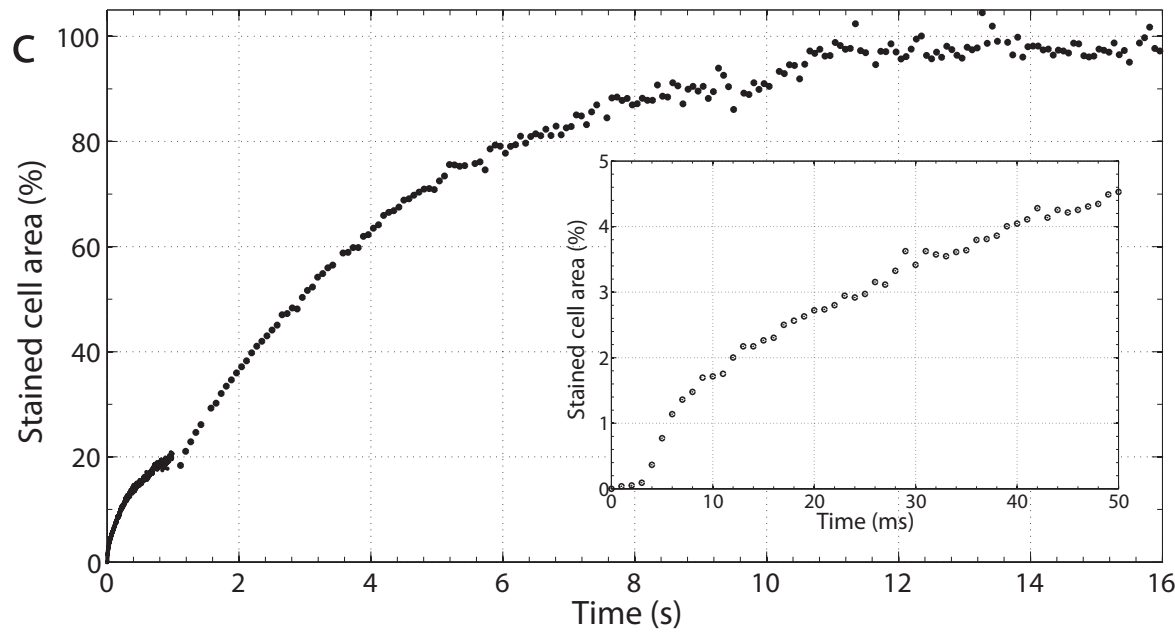

Figure 5.6: Example of an experiment. a) Several frames from the first second after insonation. b) Bright field image of the cell and bubble before ultrasound and several frames from the low-speed fluorescence recording. The red line indicates the cell perimeter and the blue square represents the cell area shown in a. c) The measured area-time curve. 


\subsubsection{Origin of PI uptake}

It was assumed that microbubble oscillations cause very local cell membrane permeabilization. To verify this assumption the origin of PI uptake in the cell was measured relative to the position of the microbubble. From the first frames of the high-speed recordings, showing a fluorescence signal from PIstained nucleic acids, the position of the source was determined. This was done by tracing the front of the fluorescence signal backwards in time to obtain the source position. For instance, in figure 5.6la, the circular stain visible at 25, 50 and $100 \mathrm{~ms}$ can be traced back to a single point of origin close to the center position of the bubble. This procedure was followed for all experiments. The distance between the bubble center and the origin of the fluorescence signal was calculated and the probability density function is plotted in figure 5.0 on the scale of a typical endothelial cell.

The average distance between the position of the bubble and the source point of the fluorescence signal ( $\left.D_{\text {bubble-fluor }}\right)$ is $1.7 \mu \mathrm{m}$. This value is smaller than the average bubble radius $(2.3 \mu \mathrm{m})$, indicating that the membrane permeabilization occurs locally and uniquely at the position of the microbubble. As expected no orientation dependence was observed, nor a dependence on the bubble radius (data not shown). However, the bubble size is expected to have an influence on the pore size, which is quantified in section 5.3.6.

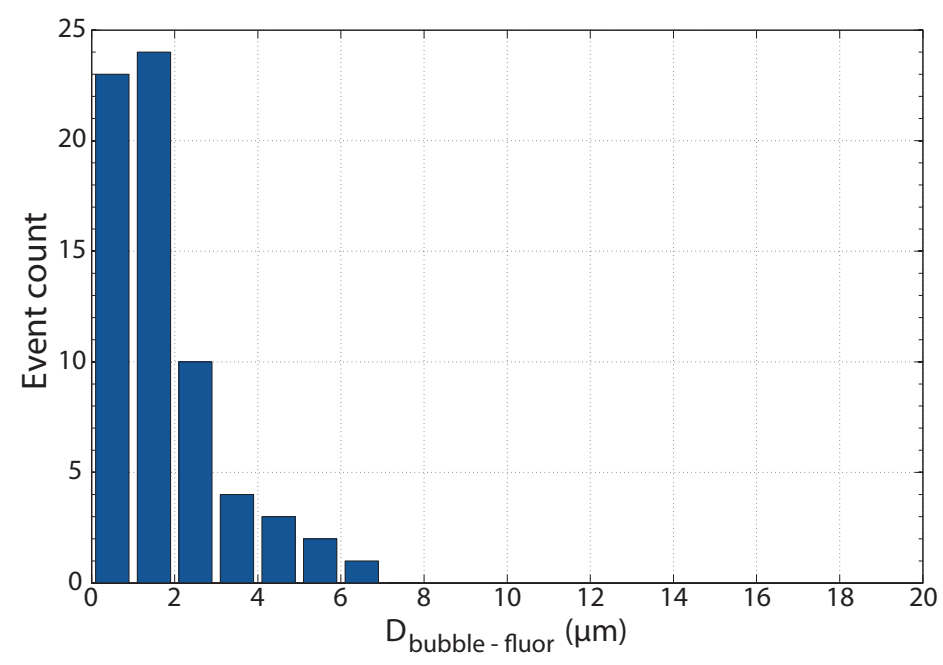

Figure 5.7: Probability density function of the distance between the bubble center and the origin of the fluorescence signal of PI uptake. The mean distance $(1.7 \mu \mathrm{m})$ is smaller than the the average bubble radius $(2.3 \mu \mathrm{m})$. 


\subsection{RESULTS}

\subsubsection{Timescale of pore formation}

Figure 5.8 shows the area-time curves derived from the high-speed fluorescence recordings of all porated cells for the first $100 \mathrm{~ms}$ after insonation. The variation in the growth rate is substantial and some cells show no increase in the stained area at this timescale. From these graphs, no correlation was found between the growth rate and the applied acoustic pressure. The average area-time curves show a minor increase in stained area as a function of the ultrasound pulse length for each individual pressure setting, however, the spread in the data is too large for it to be significant.

Most curves show an initial phase on the order of $10 \mathrm{~ms}$ where the growth rate of the stained area increases until it reaches a steady value and the area grows almost linearly. The curves of figure $5.8 \mathrm{f}$ in particular show this behavior. This can be an indication that the pore is not formed instantly at its maximum size during the microbubble oscillations, but that the pore size grows over the timescale of milliseconds.
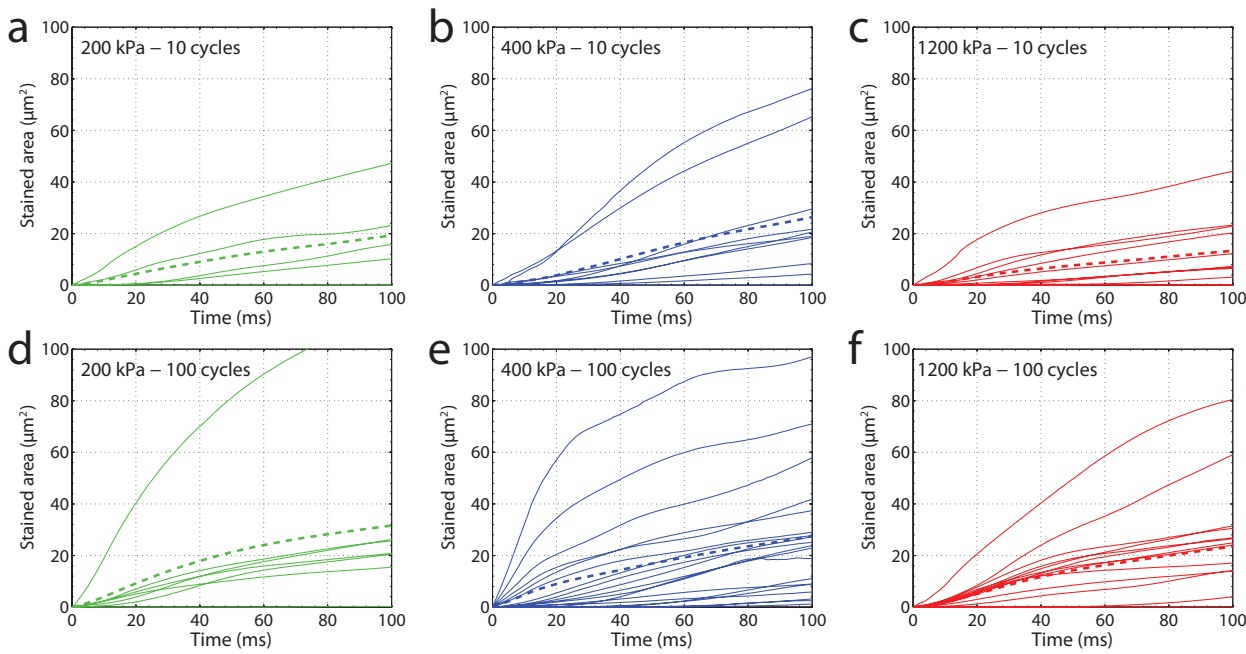

Figure 5.8: a-f) Area-time curves derived from the high-speed fluorescence recording. At $\mathrm{t}=0$, the ultrasound was applied, after which the area stained by PI was traced. The solid lines represent individual experiments and the dotted line indicates the mean for each combination of pressure and number of cycles. 


\section{HIGH-SPEED FLUORESCENCE IMAGING OF SONOPORATION}

\subsubsection{Time evolution of stained area}

For all porated cells, the growth rate of the cell area stained by PI was determined from the low-speed fluorescence recordings. The scatter plot in figure 5.9la shows the initial growth rate as a function of the bubble size. No correlation was found between the applied acoustic pressure and the growth rate, nor does the number of cycles seem to play a role in the rate at which PI spreads throughout the cell.

However, when we only consider the bubble size, we can see a clear trend in the growth rate, as shown in the histogram in figure $5.9 \mathrm{~b}$ : for an increasing bubble radius up to $3 \mu \mathrm{m}$ the area growth rate becomes higher. For even larger radii the growth rate is still high compared to the growth rate at radii smaller than $2 \mu \mathrm{m}$, however, the number of data points for large bubbles is limited.

Microbubbles display a strong resonance behavior as a function of the driving frequency. Even though the bubbles are coated with phospholipids, the applied peak-negative pressures will result in large amplitude oscillations and the microbubble can be considered to behave as if it were a free gas bubble [3I] For a free gas bubble, the resonance frequency can be approximated by the Minnaert frequency [100]. A driving frequency of $1 \mathrm{MHz}$ then results in a resonance radius of $3.3 \mu \mathrm{m}$, at which the amplitude of oscillation can be expected to be maximum. This corresponds well with the peak in the histogram of figure $5.9 \mathrm{~b}$, implying that the highest amplitude of oscillation causes the largest pore size in the cell membrane and consequently the highest growth rate of the stained area. The asymmetry of the graph could be attributed to the fact that larger bubbles have an increased contact area with the cell membrane and cause more fluid flow than smaller bubbles, and thereby induce a larger pore size.
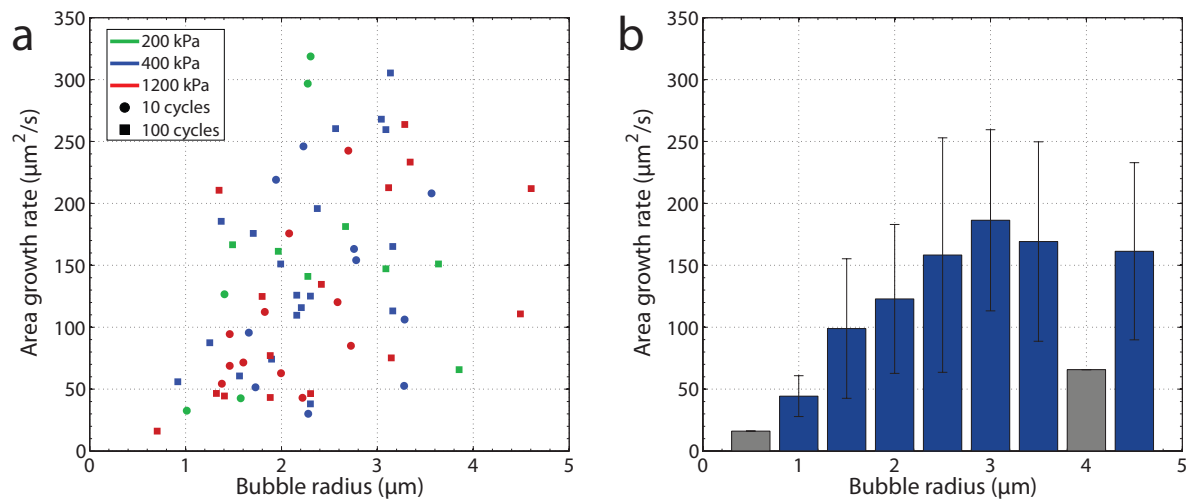

Figure 5.9: a) Scatter plot of the initial growth rate of the area stained by PI. b) Histogram of the initial growth rate. Gray bars indicate single measurements. 


\subsection{RESULTS}

\subsubsection{Pore closure}

Zhou et al. [82] and Van Wamel et al. [24] showed that bubble-induced pores can be closed again within several seconds after sonoporation. Compared to the situation where the pore is formed and stays open, this will yield different time evolutions of the stained area, the total fluorescence intensity of the cell and the fluorescence intensity level of the nucleus. In figure $5.10 \mathrm{la}-\mathrm{c}$, these quantities are shown for a cell represented by the numerical model for three different values of $t_{\text {close }}$. The length of the cell is $30 \mu \mathrm{m}$, with the nucleus located in the middle of the cell. The concentration of PI outside the cell, $c_{0}$, was set to one. The diffusion constant $D$ was estimated from a low speed recording to be approximately $5 \mu \mathrm{m}^{2} / \mathrm{s}$. The stained area was determined by taking the percentage of grid points with a concentration above a certain detection threshold level. This value was estimated from the experimental data for the fluorescence signal emitted by the cell cytoplasm and taken to be 0.1 .

If the pore does not close, the total fluorescence intensity and the intensity at the location of the nucleus will continue to grow, as PI keeps on flowing into the cell until an equilibrium is reached. The detectable concentration level is much lower than the equilibrium value. When the pore has just been formed, $\mathrm{PI}$ flows into the cell and stains the complete area of the cell, after which the fluorescence intensity continues to increase until the equilibrium is reached.
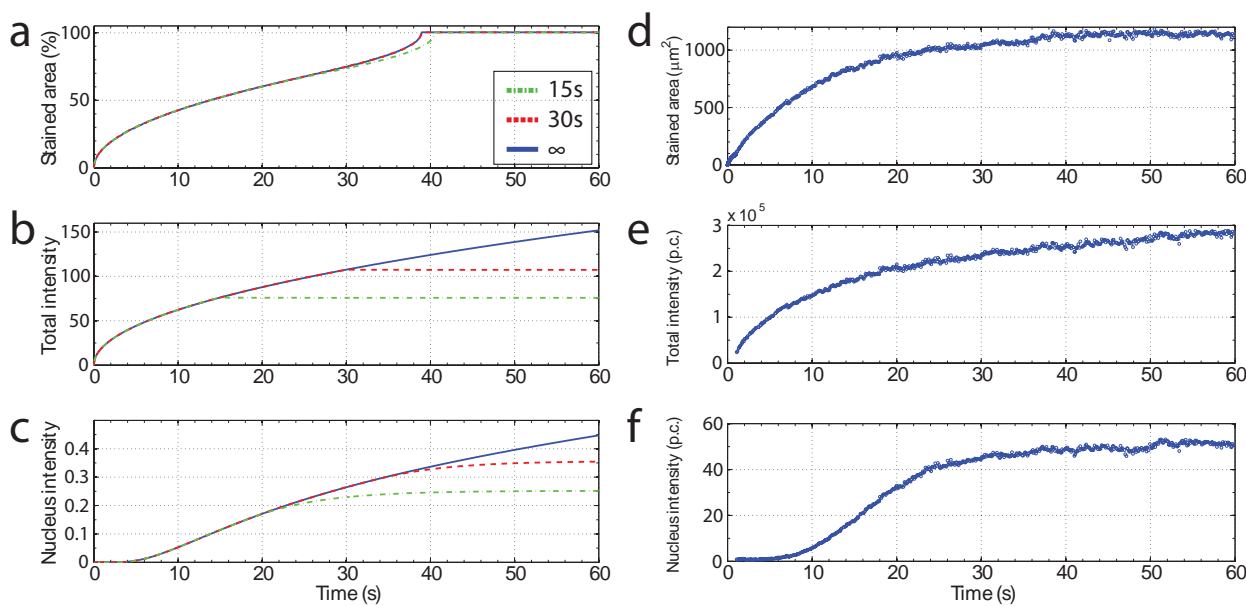

Figure 5.10: The stained area of a cell, the total fluorescence signal emitted by the cell and the fluorescence intensity at the position of the nucleus as a function of time for $(a-c)$ a cell represented by a one-dimensional numerical model with different pore opening times and (d-f) a typical sonoporation experiment. 
When the pore closes after several seconds, the total fluorescence intensity and the nucleus intensity will reach a steady state before the concentration inside has reached the same level as the concentration outside the cell. This steady state is now reached on a similar timescale at which the stained area reaches its maximum.

Figure $5.10 \mathrm{~d}-\mathrm{f}$ show the same quantities obtained from a typical experiment. All curves reach a steady-state on the same timescale, indicating that the formed pore is closed within the time frame of this experiment. This was confirmed by a comparison of the fluorescence intensity of the nucleus for porated cells and apoptotic cells in general. The latter emit a much stronger fluorescence signal, at least a factor 2 higher.

When the fluorescence signal from the cell nucleus reaches this type of steady state, the bubble-induced pore must be closed. Figure 5.11 la shows the time for the nucleus to reach its maximum intensity as a function of the distance between the bubble and the nucleus. On average the maximum is reached in 40-50 seconds after insonation, indicating the maximum time window for uptake by the cell.

In addition, figure $5.17 \mathrm{~b}$ shows the maximum intensity of the cell nuclei. For a larger distance between the bubble and the nucleus, the maximum observed intensity and thus the amount of $\mathrm{PI}$ in the cell nucleus is clearly lower. This can be attributed to the fact that the PI molecules encounter more binding sites in the cytoplasm before reaching the nucleus.
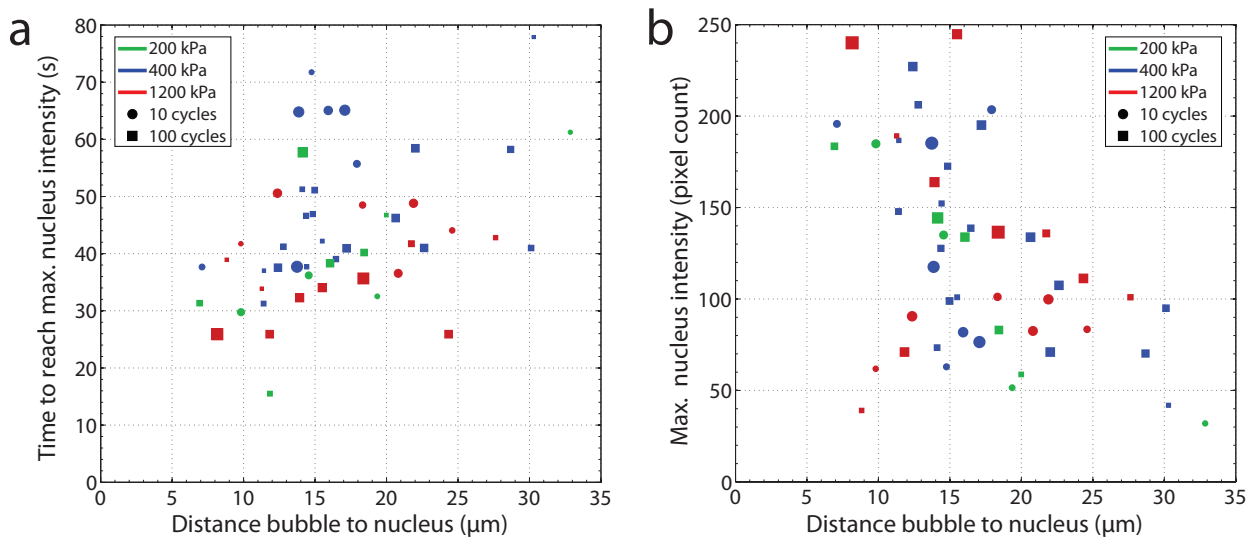

Figure 5.11: a) The time required to reach the maximum nucleus staining intensity. The relative size of each marker corresponds to the microbubble radius. b) The maximum nucleus staining intensity as a function of the distance between the microbubble and the nucleus. 


\subsection{CONCLUSIONS AND DISCUSSION}

\subsubsection{Bubbles interacting with multiple cells}

During the experiments, the microbubbles floated up against the cell layer on the Opticell $^{\text {TM }}$ membrane. Due to the height profile of the HUVECs (see figure $5.1 \mathrm{~b}$ ), many bubbles resided in between two cells near or at the cell border. As a result, single microbubbles were able to induce sonoporation in two adjacent cells. Double sonoporation was observed in $20 \%$ of the experiments.

An example of a double sonoporation event is shown in figure $5.12 \mathrm{~b}$. A microbubble located at the cell border between two neighboring cells causes uptake of $\mathrm{PI}$ in both cells. In figure 5.12la a similar configuration is depicted, however, the microbubble only causes uptake of PI in one of the cells, as can be seen from the fluorescence images.

a

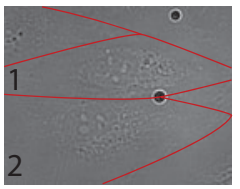

Before US

b

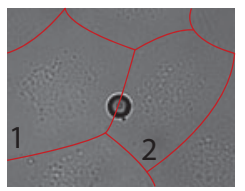

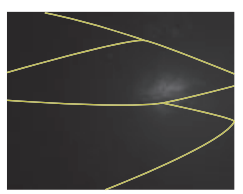

$\mathrm{t}=3 \mathrm{~s}$

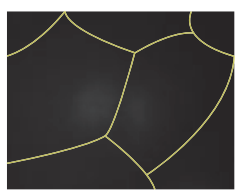

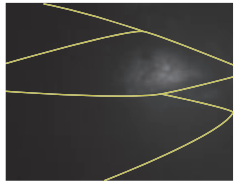

9s

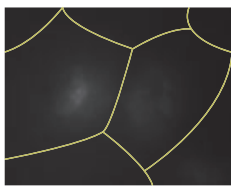

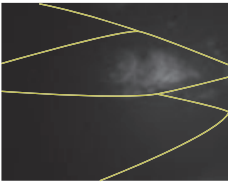

$17 \mathrm{~s}$

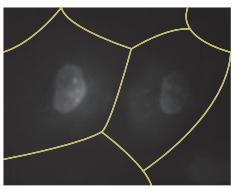

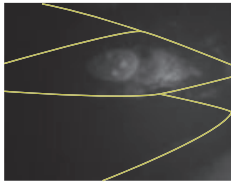

$50 \mathrm{~s}$

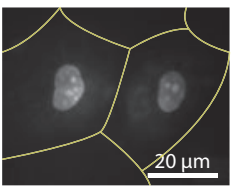

Figure 5.12: Two examples of a sonoporation event ( $400 \mathrm{kPa}, 10$ cycles). The bright field images show the cells before insonation (red/yellow lines indicate cell borders). The fluorescence images indicate the uptake of PI over the course of minute. The nuclei clearly display the highest fluorescence signal. a) A microbubble located in between two cells only causes sonoporation in cell 1. b) A microbubble in between two cells causes sonoporation in both cells.

\subsection{Conclusions and discussion}

This study shows that time-resolved fluorescence imaging of the uptake of PI by an endothelial cell yields valuable information about the physical mechanisms of sonoporation. The high-speed recordings revealed that the bubble-induced pores are created very locally, as staining of RNA in the cytoplasm is confined to the position of the microbubble in the first milliseconds after insonation before the fluorescence signal spreads throughout the cell. Due to a high fluorescence background emitted by the medium, the presence of a microbubble 'shadow' 
blocked the fluorescence signal emitted by the region of the cytoplasm located above the bubble, thereby reducing the spatial resolution in the first milliseconds after sonoporation. This problem could be solved partially by switching to a phenol red free cell culture medium or a PBS-solution containing $\mathrm{PI}$, to reduce the background fluorescence intensity.

In the majority of the experiments, a fluorescence signal is detected in the first millisecond after the microbubble oscillations. Although the bubble image can interfere with the observation of the fluorescently marked area in the first milliseconds, a slow startup phase is seen for an extended period up to $30 \mathrm{~ms}$ after insonation, indicating that the pore is not formed instantaneously at its maximum size. In some cells fluorescence is not detected until $100 \mathrm{~ms}$ or longer. It could be that for these cells the pore formation takes place on a very long timescale, however it is more likely that the pore was formed in a region of the cytoplasm where the concentration of RNA was too low to be detectable on the order of milliseconds.

The time evolution of the stained area of a cell indicates that the inflow of $\mathrm{PI}$ is diffusion-driven. Furthermore, the results show that larger microbubbles and microbubbles with a large oscillation amplitude produce a larger pore, as seen from the flow rate at which PI enters and spreads throughout the cell. The applied acoustic pressure or number of cycles does not play an important role in the uptake following insonation for the pressures between $200-1200 \mathrm{kPa}$. However, the efficacy of sonoporation is increased for higher pressures. Since the microbubble behavior is strongly influenced by the nearby cell layer and Opticell $^{\mathrm{TM}}$ wall [22, 101] , and the resonance frequency of a microbubble changes with the amplitude of oscillation [31, 102], a prediction of the exact bubble response to the ultrasound is difficult to make. By combining ultra-high-speed imaging of the bubble oscillations with time-resolved fluorescence imaging of the uptake of PI, an improved correlation can be found of the bubble size and oscillation amplitude with the dynamics of PI molecules inside the cell.

At a peak-negative pressure of $1200 \mathrm{kPa}$, microbubbles are known to be able to produce microjets [83], which would give rise to a more advection-driven process. However, the jet produced by a bubble is directed towards the nearby boundary, which in this case is the lower surface of the endothelial cell that is parallel to the Opticell ${ }^{\mathrm{TM}}$ membrane. Since the orientation of the endothelial cell is perpendicular to this surface and the recordings were made using a top-view, a fast injection of PI molecules is unlikely to be seen with this setup. When no net driving force is present, fluid flow on a micrometer length scale is dominated by viscosity, and the applied ultrasound had a maximum duration of $100 \mu$ s, i.e. much shorter than the temporal resolution of the high-speed recordings. A side-view setup [52] that is able to capture the plane in which the jet occurs at 


\subsection{CONCLUSIONS AND DISCUSSION}

the relevant timescale can provide more insight into this phenomena.

The time-resolved information about the stained area, the total fluorescence intensity and the intensity of the nucleus provides insight into the timescale on which the formed pore closes. For ultrasound-triggered drug delivery applications the time a pore is open is essential as it determines the window for enhanced uptake by the vascular endothelial cells. The results show that pores are opened for a period up to a minute approximately, after a single ultrasound pulse. The accuracy of these measurements can be enhanced when the experimental results can be matched to a better, two-dimensional numerical model, based on reliable values for the diffusion constant of $\mathrm{PI}$ inside a cell and the pore size. Experiments in this direction are underway.

The presented experiments were performed under static conditions, however, by performing sonoporation experiments under flow conditions a more realistic setting is created, which is also beneficial for the proliferation of HUVECs, due to the applied dynamic shear stress. It will enable a more accurate determination of the pore opening time, by adding the $\mathrm{PI}$ at a certain time point following the insonation. These type of experiments were already performed by Van Wamel et al. [24], however not at the scale of a single cell. 


\section{6}

\section{Characterization of polymeric microcapsules for contrast-enhanced photoacoustic imaging}

Polymeric microcapsules were developed to serve as multimodal contrast agents for photoacoustic imaging and ultrasound imaging. Furthermore, the capsules can be loaded with lipophilic drugs to provide local release upon insonation or optical activation. The shell of the capsules was doped with Nile Red to assist absorption of the laser irradiation. The capsules are susceptible both to pulsed and continuous laser excitation. Single microcapsules generate an enhanced acoustic signal as compared to traditional contrast agent nanoparticles. The acoustic signal is produced by the growth and collapse of a vapor bubble that is generated by the heat transfer from the polymer shell to the surrounding water. The response of the capsule to the laser irradiation was modeled and found to be in good agreement with the experimentally obtained results. 


\subsection{INTRODUCTION}

\subsection{Introduction}

Medical ultrasound imaging is a cost-effective, non-invasive and flexible imaging modality that can be used at the bedside of the patient and offers time-resolved imaging of various internal structures of the body. Diagnostic ultrasound is employed in general for organ perfusion imaging and can be used to determine the endocardial border delineation and the intima media thickness of the carotid. Just as magnetic resonance imaging or X-ray imaging, ultrasound imaging has a relatively low spatial resolution, as it is limited in resolution by the wavelength of the acoustic waves ( $\sim 1 \mathrm{~mm})$.

Photoacoustic imaging is a diagnostic imaging modality that offers a similar spatial resolution compared to ultrasound imaging. The photoacoustic effect is based on the absorption of pulsed laser light in biological tissue. The resulting thermal expansion produces an ultrasonic wave that is detected by an ultrasound receiver. The high absorption of hemoglobin in the blood allows for imaging of the vascularization of tissue. Furthermore, photoacoustic imaging can be used to determine the level of oxygenation of the blood as oxygenated and deoxygenated hemoglobin have different absorption spectra. Photoacoustic imaging has a wide field of applications such as brain lesion detection [103], hemodynamics monitoring [104], intravascular imaging of atherosclerosis [105] and breast cancer diagnostics [106]. The imaging depth is low compared to ultrasound imaging, as the scattering of the laser light is substantial at a depth of several centimeters and the maximum permissible exposure (MPE) for nanoseconds laser pulses is approximately $100 \mathrm{~mJ} / \mathrm{cm}^{2}$ in the near-infrared.

To increase the absorption of the applied laser irradiation while staying below the MPE, plasmonic metal nanoparticles have been investigated as contrast agents in photoacoustic imaging [107, 108]. Their absorption cross-section is orders of magnitude higher than the cross-section of hemoglobin. The resulting thermal expansion is increased, leading to an enhanced ultrasound signal at higher penetration depths. When injected into the bloodstream, the nanoparticles increase the blood-to-tissue contrast. The enhanced visualization of blood vessels is employed in the detection of tumorous tissue, which is highly vascularized. Furthermore, nanoparticles can also be functionalized to be used a molecular contrast agents.

In medical ultrasound imaging, contrast agents are also used to enhance the blood-to-tissue contrast. Ultrasound contrast agents (UCAs) consist of gas-filled, coated microbubbles $(1-10 \mu \mathrm{m}$ in diameter) that drastically enhance the backscattered signal of the applied sound field in organ-perfusion imaging, due to their high compressibility. Recently, UCAs have been adopted as drugcarriers for local intravascular drug delivery [8]. By incorporating drugs into the 
core or shell of the microbubbles, an ultrasound-triggered drug delivery system can be created [66, 109]. Various system designs have been reported, such as liposome-loaded microbubbles [68] and polymeric microcapsules [41, 63]. These microbubble systems can be used as contrast agents for imaging purposes at low Mechanical Index ( $\mathrm{MI})$ ultrasound and provide a local therapeutic effect upon disruption at a high MI.

Here, we present a novel multimodal contrast agent polymeric microcapsule, designed for ultrasound imaging, ultrasound-triggered drug delivery and photoacoustic imaging. Their acoustical properties have been investigated by Kooiman et al. [23]. Here, the photoacoustic capabilities are characterized and compared to existing photoacoustic contrast agent nanoparticles. The observed photoacoustic effect in the microcapsules is based on vaporization instead of thermal expansion and the underlying physical mechanism is modeled. In addition to the traditional pulsed excitation of the agent, continuous wave (CW) irradiation was found to generate a strong acoustic signal for the full duration of the exposure to laser light.

\subsection{Experimental parameters}

\subsubsection{Contrast agents}

The microcapsules were produced by Philips Research using ink-jet printing technology as described by Böhmer et al. [41]. The shell of the capsules consists of PLA-PFO (Poly(L-lactic acid) terminated by $1 \mathrm{H}, 1 \mathrm{H}$-perfluorooctyloxycarbonyl) [170]. Two types of capsules were studied with different core contents: oil-filled capsules were made, incorporating hexadecane, and gas-filled capsules containing nitrogen. Figure 6 . Jla shows a SEM image of gas-filled capsules. The produced capsules had very narrow size distributions with a mean radius of $3 \mu \mathrm{m}$.

The fluorescent dye Nile Red (NR) was incorporated into the shell material at a concentration of $0.1 \% \mathrm{w} / \mathrm{w}$ to increase the optical absorbance whilst leaving the mechanical properties of the polymer unaffected. The confocal image of a capsule in figure $\mathbf{6 .}$. Ib clearly shows the location of the dye inside the shell. The shell thickness, as calculated from the size of the capsule and the oilpolymer ratio, was found to be $600 \mathrm{~nm}$, which matches the confocal recording. The maximum absorption of the doped shell material is $530 \mathrm{~nm}$, which was measured using a spectrometer. 


\subsection{EXPERIMENTAL PARAMETERS}
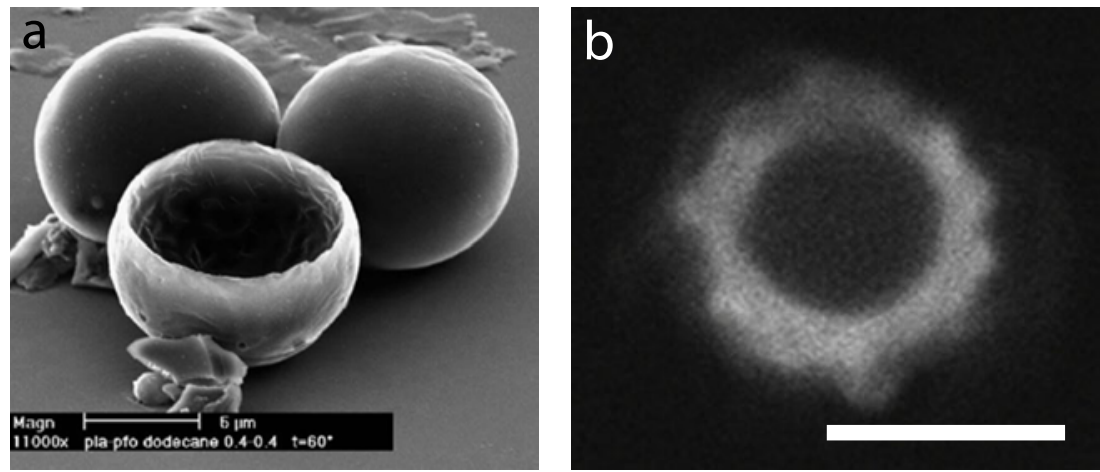

Figure 6.1: a) SEM image of hollow microcapsules. b) Confocal microscopy image of an oil-filled microcapsules. Scale bars represent $5 \mu \mathrm{m}$.

Gold nanospheres with a mean diameter of $25 \mathrm{~nm}$ were purchased from Aurion (Wageningen, The Netherlands). The nanospheres have a maximum absorption at $520 \mathrm{~nm}$, to match the wavelength of the lasers.

\subsubsection{Experimental setup}

To simultaneously study the optical and acoustical response of the microcapsules, a diluted suspension of the capsules $\left(1 \cdot 10^{4} / \mathrm{mL}\right)$ was injected into an Opticell $^{\mathrm{TM}}$. The Opticell ${ }^{\mathrm{TM}}$ was placed in a water bath, located underneath an upright fluorescence microscope setup, depicted in figure 6.2. The microscope was equipped with a $60 \times$ water-immersion objective (NA $=0.9$, Olympus, The Netherlands).

For pulsed laser irradiation a frequency-doubled, Q-switched Nd:YAG laser was used (Nano-PIV, Litron Lasers, UK) with a pulse duration of $6 \mathrm{~ns}$ and a maximum pulse energy of $200 \mathrm{~mJ}$ at a wavelength of $532 \mathrm{~nm}$. The pulse energy was controlled by the internal attenuator and additional neutral density filters. A 5 Watt DPSS laser (Cohlibri, Lightline, Germany) with an output wavelength of $532 \mathrm{~nm}$ was employed for continuous laser irradiation. The laser output of the CW laser was gated in time by an acousto-optic modulator (AOM) and the amplitude was controlled by the internal power settings.

At the bottom of the water bath a $1 \mathrm{MHz}$ focused transducer (focal length $=4 \mathrm{~cm})($ C302, Panametrics, USA) was located to record the acoustical signals emitted by the events. The acoustic signals were amplified using a preamplifier (5077PR, Panametrics) to enhance the sensitivity. The traces were recorded with an digital storage oscilloscope (DPO3034, Tektronix, UK). Due 
to the proximity of the microscope objective to the irradiated microcapsules, an acoustic reflection from the objective was present in the acoustic recordings. This reflection was filtered in Fourier space from all recordings.

The laser light was focused onto the sample through the microscope objective. The very same objective was used to image the phenomena and optical recordings were made with the Brandaris 128 ultra-high-speed camera [15] at frame rates ranging from 2-10 million frames per second (Mfps). The optical and acoustical focus were co-aligned using a $0.2 \mathrm{~mm}$ PVDF needle hydrophone (Precision Acoustics Ltd., UK). A similar setup was used by Sijl et al. [50] to simultaneously record the acoustical and optical response of an ultrasound contrast agent to an applied ultrasound field.

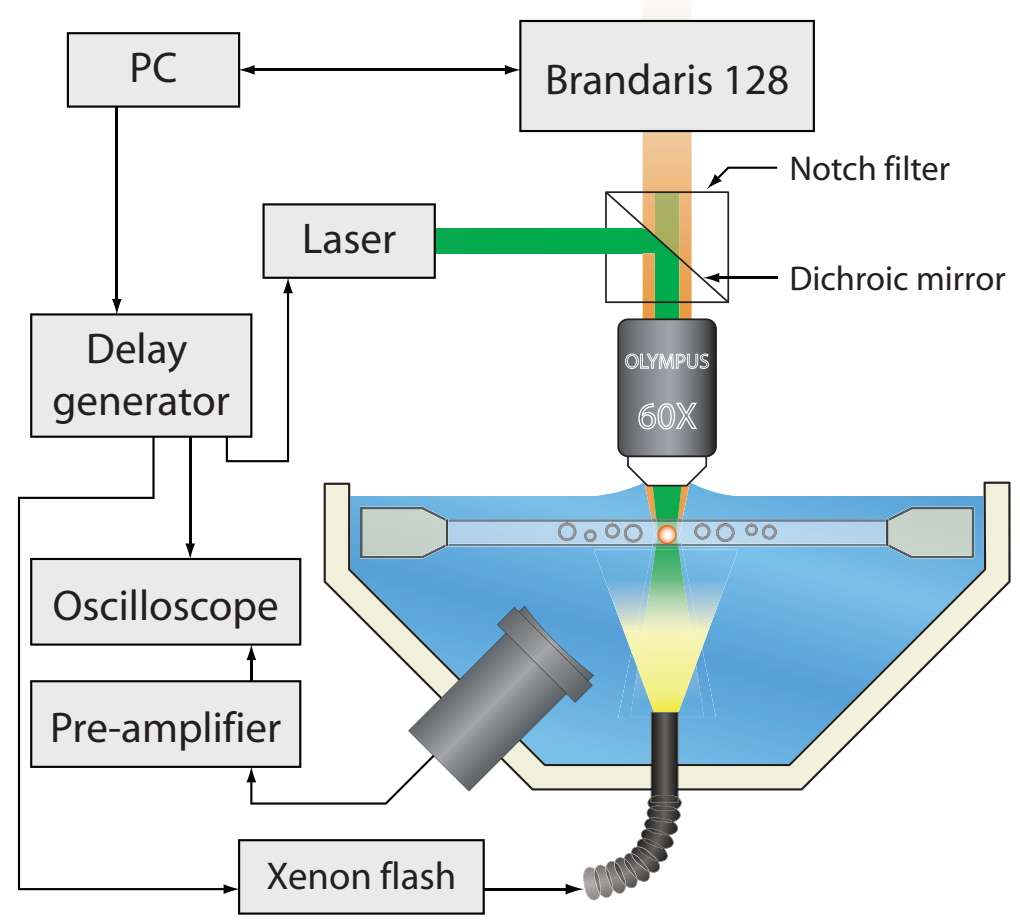

Figure 6.2: Schematic representation of the experimental setup. The Brandaris 128 ultra-high-speed camera and a laser are coupled to a combined optical/acoustical setup. The microscope objective is immersed in a water bath including an Opticell ${ }^{\mathrm{TM}}$ to contain the microcapsules and gold nanospheres, an ultrasound transducer to measure the acoustic signals and an optical fiber for bright field illumination. 


\subsection{EXPERIMENTAL RESULTS}

\subsection{Experimental results}

\subsubsection{Pulsed excitation}

The optical and acoustical response of oil-filled microcapsules to different laser fluences was recorded. Figure 6.3 shows several sample frames from the optical response of an oil-filled microcapsule irradiated at a laser fluence of $72 \mathrm{~mJ} / \mathrm{cm}^{2}$. In the top right corner of the capsule a small part of the polymer shell is fractured due to the absorption of the laser light by the NR molecules. A vapor bubble emerges and collapses. After the collapse, the capsule-water interface is forced into the capsule before its equilibrium position is retained.

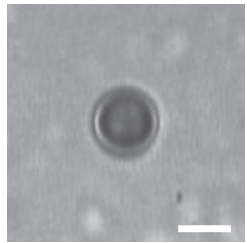

Before US

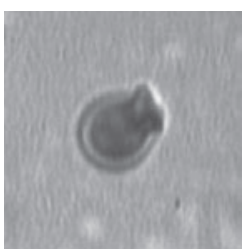

$1.0 \mu \mathrm{s}$

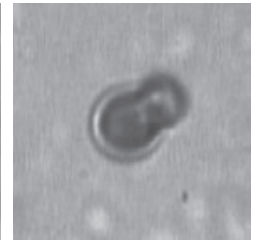

$\mathrm{t}=0.2 \mu \mathrm{s}$

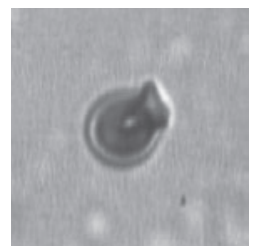

$1.2 \mu \mathrm{s}$

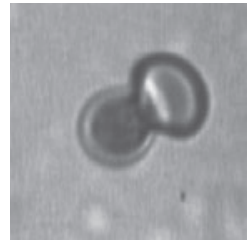

$0.4 \mu \mathrm{s}$

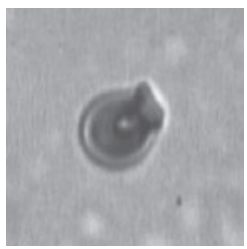

$1.4 \mu \mathrm{s}$

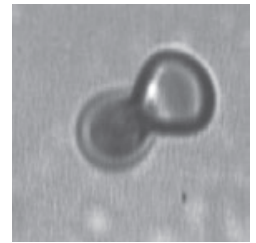

$0.6 \mu \mathrm{s}$

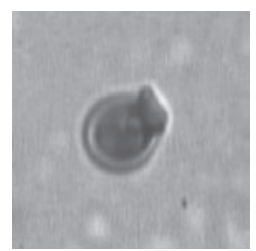

$1.6 \mu \mathrm{s}$

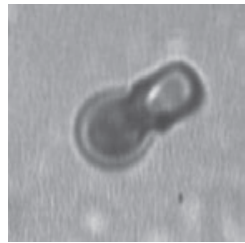

$0.8 \mu \mathrm{s}$

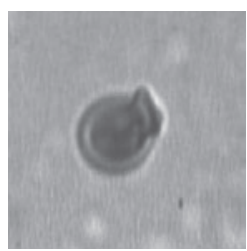

$1.8 \mu \mathrm{s}$

Figure 6.3: Image sequence of a high-speed recording (9.54 Mfps) of an oil-filled capsule irradiated by a $72 \mathrm{~mJ} / \mathrm{cm}^{2}$ laser pulse. Scale bar represents $5 \mu \mathrm{m}$.

The activation threshold for the microcapsules was found to be $60 \mathrm{~mJ} / \mathrm{cm}^{2}$. When laser fluences just above this threshold are applied, the capsule stays intact except for a single point on the capsule wall where the polymer is damaged and a vapor bubble arises.

Figure 6.4 shows the optical and acoustical response of an oil-filled microcapsule irradiated at a fluence of $1.44 \mathrm{~J} / \mathrm{cm}^{2}$. When the laser light hits the capsule, the shell is violently disrupted while a vapor bubble is formed that grows to its maximum radius in approximately $1 \mu \mathrm{s}$ while fragments of the shell surround parts of the bubble. When the maximum radius is reached, the vapor condensates and the bubble collapses, leaving fragments of the polymer shell. From the optical recording the radius-time curve was determined, depicted in figure $6.4 \mathrm{~b}$. The measured acoustical response, representing the pressure am- 
plitude at the transducer surface, is shown in figure 6.4c. This trace shows a pressure pulse from the rapid bubble growth followed by a rarefaction wave. When the vapor collapses a second pressure pulse is generated.
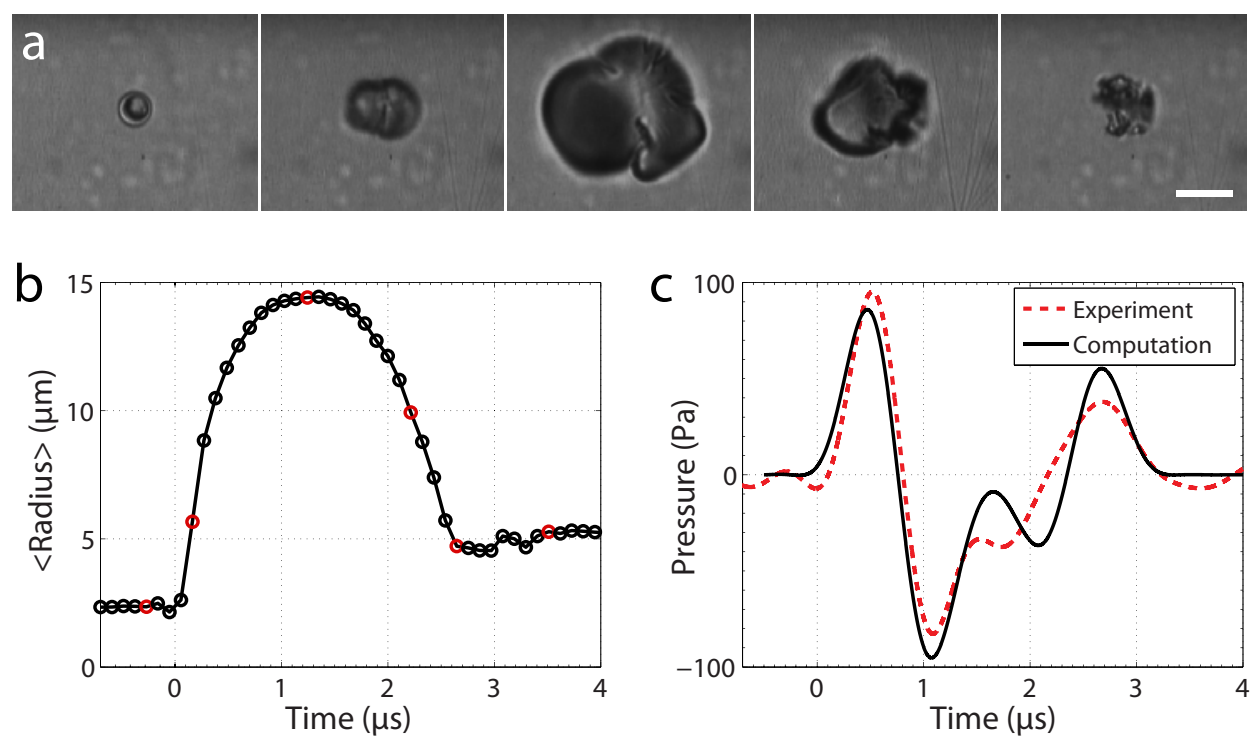

Figure 6.4: Optical and acoustical response of a microcapsule to a $1.44 \mathrm{~J} / \mathrm{cm}^{2}$ laser pulse, applied at $t=0$. a) Sample frames from the high-speed recording, the frames correspond to the red circles in $\mathrm{b}$, scale bar represents $10 \mu \mathrm{m}$ b) Radius-time curve of the vapor bubble (laser pulse at $t=0$ ). c) Acoustical signal measured with the transducer (dashed line). The solid line depicts the predicted pressure, corrected by a factor of 3.

The acoustic pressure emitted by the bubble can be related to the radial oscillations of the bubble, as described by Leighton [III]:

$$
P(r, t)=\rho_{w} \frac{R(t)}{r}\left(R(t) \ddot{R}(t)+2 \dot{R}(t)^{2}\right) .
$$

Figure $6.4 \mathrm{c}$ also shows the pressure, calculated from the radius-time curve of the vapor bubble. The calculated pressure was normalized to the measured pressure to show the shape agreement. The computed pressure was a factor 3 higher than the measured pressure, which may be attributed to an error in the radius determination. For a radially oscillating, spherical bubble, the pressure scales with the third power of the radius [50]. An error of $45 \%$ percent in the measured radius accounts for the factor 3 . Due to the asymmetrical shape of the bubble, an error of that magnitude is possible. Furthermore, an error can exist in the 


\subsection{EXPERIMENTAL RESULTS}

absolute calibration of the ultrasound transducer, since the hydrophone used a standard for the calibration has an accuracy of $10 \%$.

The activation threshold for vaporization was determined from optical recordings. No vaporization events were observed for fluences of $50 \mathrm{~mJ} / \mathrm{cm}^{2}$ or lower and at $72 \mathrm{~mJ} / \mathrm{cm}^{2}$, all microcapsules displayed vaporization, leading to a threshold value of $60 \pm 10 \mathrm{~mJ} / \mathrm{cm}^{2}$. Furthermore, the size and lifetime of the vapor bubbles strongly depend on the applied laser energy, as shown in figure 6.5 .

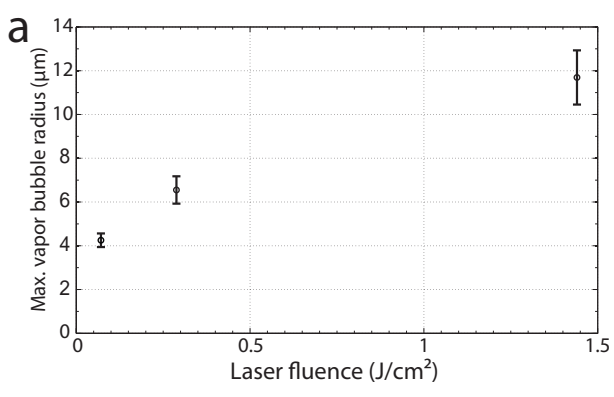

Figure 6.5: Maximum radius (a) and lifetime (b) of vapor bubbles from oil-filled microcapsules irradiated by pulses laser light.

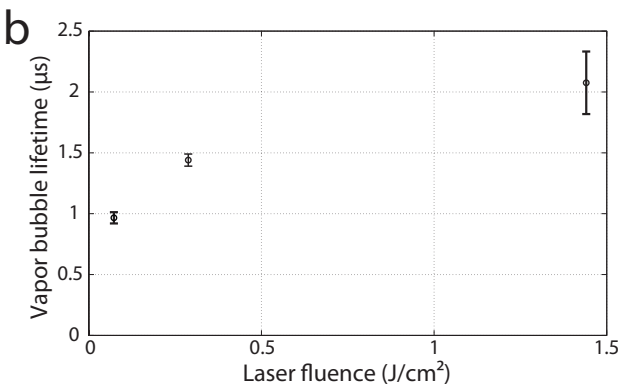

The composition of the core of the microcapsules did not significantly influence the overall response. The response to pulsed excitation of oil-filled and gas-filled microcapsules was identical as well as the typical lifetime, vapor bubble size and activation threshold. The only difference was found at the final collapse, where minor rebounds were visible for the gas-filled capsules.

The acoustical response of the oil-filled capsules was compared to the acoustical response of gold nanospheres. Figure 6.6 shows the signal-to-noise ratio (SNR), determined from the acoustical measurements, as a function of the applied laser fluence. The data from the microcapsules represent measurements of individual microcapsules, whereas the data of the nanoparticles was measured using a concentrated solution of gold nanospheres (3.3.10 ${ }^{11}$ particles $\left./ \mathrm{mL}\right)$. The spot size of the laser was adjusted to be on the order of the diameter of the microcapsule. Consequently, the nanospheres response was obtained from a comparable volume as the volume of the capsule.

The SNR obtained with single microcapsules is significantly higher than the SNR for the nanospheres solution. Furthermore, at laser fluences above $280 \mathrm{~mJ} / \mathrm{cm}^{2}$, vaporization was also observed in the nanospheres solution. An example of the vaporization from gold nanospheres irradiated at a laser fluence of $1.44 \mathrm{~J} / \mathrm{cm}^{2}$ is depicted in figure 6.0 . 


\section{MICROCAPSULES FOR PHOTOACOUSTIC IMAGING}

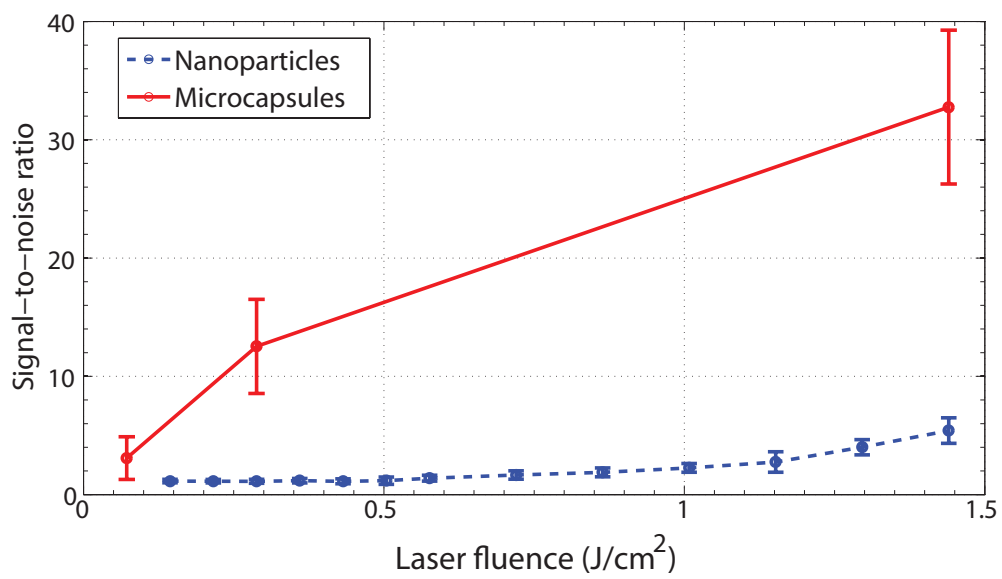

Figure 6.6: Comparison of the signal-to-noise ratio of the measured acoustical response of single oil-filled microcapsules and a solution of gold nanospheres.

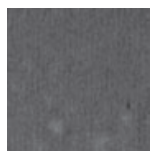

$\mathrm{t}=0 \mathrm{~s}$

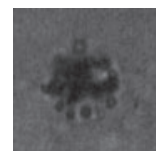

$66 n s$

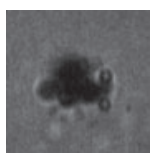

$132 \mathrm{~ns}$

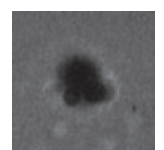

198ns

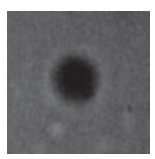

$264 n s$

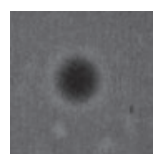

330ns

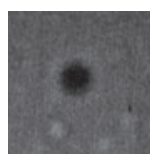

$396 n s$

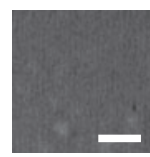

$462 n s$

Figure 6.7: Vaporization in a solution of gold nanospheres at a laser fluence of $1.44 \mathrm{~J} / \mathrm{cm}^{2}$, scale bar represents $5 \mu \mathrm{m}$.

\subsubsection{Continuous excitation}

When the capsules were irradiated by continuous laser light, the vapor formation did not take place instantaneously, as with the pulsed excitation. Figure $\mathbf{6 . 8}$ shows several sample frames from a high-speed recording of an oil-filled capsule excited at a laser intensity of $5 \mathrm{MW} / \mathrm{cm}^{2}$ for $45 \mu \mathrm{s}$. First a strong fluorescent signal is emitted by the capsule, while the shell is being heated, after which the vaporization is initiated. The vapor continues to grow up to a maximum diameter of $8 \mu \mathrm{m}$. When the laser is turned off, the vapor collapses, leaving a deformed capsule.

At high continuous laser intensities, maintained oscillations of the vapor bubble were observed, following the initial growth. Figure 6.9la depicts the evolution of an oil-filled capsule irradiated at a laser intensity of $12 \mathrm{MW} / \mathrm{cm}^{2}$. After the vapor formation, the vapor bubble grows asymmetrically before the oscillations 


\subsection{EXPERIMENTAL RESULTS}

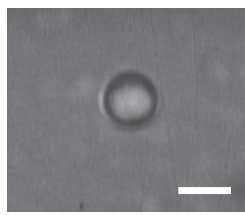

Before US

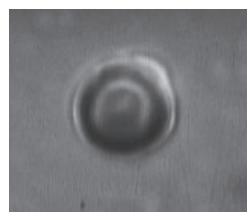

$25 \mu \mathrm{s}$

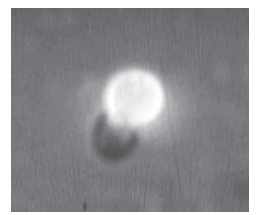

$\mathrm{t}=3 \mu \mathrm{s}$

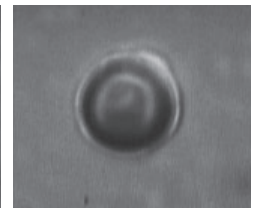

$40 \mu \mathrm{s}$

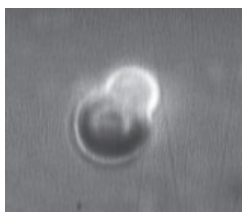

$5 \mu \mathrm{s}$

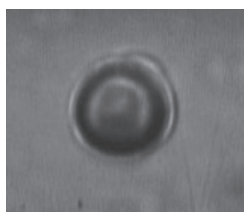

$45 \mu \mathrm{s}$

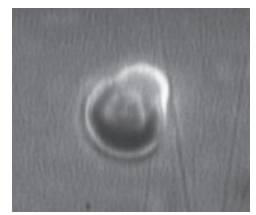

$10 \mu \mathrm{s}$

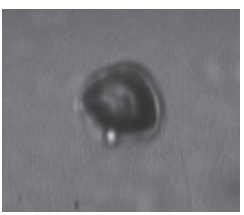

$46 \mu \mathrm{s}$

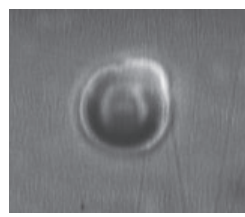

$15 \mu \mathrm{s}$

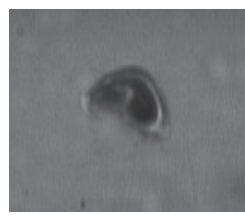

$48 \mu \mathrm{s}$

Figure 6.8: Image sequence of a high-speed recording (2.1 Mfps) of an oil-filled capsule exposed to $5 \mathrm{MW} / \mathrm{cm}^{2}$ continuous laser irradiation starting at $t=0$ with a duration of $45 \mu \mathrm{m}$. Scale bar represents $5 \mu \mathrm{m}$.

become stable. Shell fragments can be seen at the vapor/liquid interface. Finally when the laser output is stopped, the bubble collapses and the shell fragments remain.

Figure 6.9c shows the measured acoustic signal, which is on the same order as the signals obtained from microcapsules excited by a pulsed laser at $1.44 \mathrm{~J} / \mathrm{cm}^{2}$. Figure $6.9 \mathrm{~d}$ shows the frequency content of the acoustic response containing a fundamental frequency of approximately $0.3 \mathrm{MHz}$ and pronounced higher harmonics. The frequency content of the calculated pressure derived from the radius-time is plotted as well. The fundamental and second harmonic components are in good agreement with each other.

The threshold for the formation of a vapor bubble using continuous excitation was derived from the optical recordings and found to be $1.5 \pm 0.5 \mathrm{MW} / \mathrm{cm}^{2}$. At $1 \mathrm{MW} / \mathrm{cm}^{2}$ no vaporization was observed, however, the shell morphology was affected by the laser irradiation. Up to $12 \mathrm{MW} / \mathrm{cm}^{2}$ no oscillations of the vapor bubble were observed, resulting in an equilibrium radius between 5 to $10 \mu \mathrm{m}$. Intensities above $12 \mathrm{MW} / \mathrm{cm}^{2}$ always resulted in maintained oscillations of the vapor during laser irradiation. 

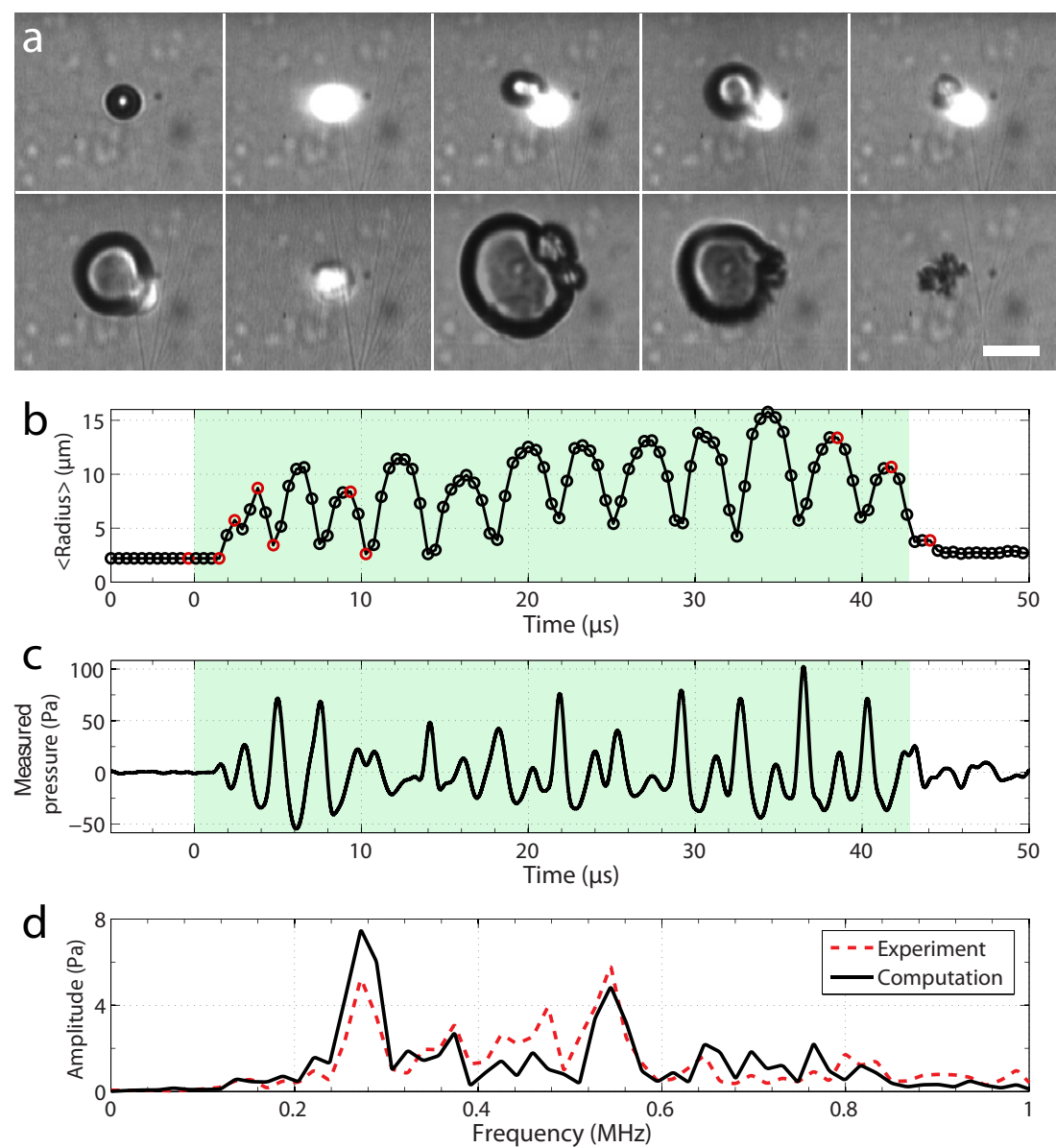

Figure 6.9: Optical and acoustical response of a microcapsule excited by continuous laser irradiation $\left(12 \mathrm{MW} / \mathrm{cm}^{2}\right)$. a) Sample frames from the high-speed recording (2.2 Mfps), the frames correspond to the red circles in b, scale bar represents $10 \mu \mathrm{m}$. b) Radius-time curve of the vapor bubble (laser pulse indicated by marked area). c) Acoustical signal measured with the transducer. d) Frequency content of the measured acoustic signal (dashed line). The solid line represents the frequency content of the calculated acoustic response.

\subsection{Modeling of the vaporization process}

The laser-induced vaporization was modeled by setting up the energy balance for a laser-induced vapor bubble, as depicted schematically in figure 6.10. Part of the the laser energy is absorbed by the doped polymer shell $\left(W_{a b s}\right)$. We 


\subsection{MODELING OF THE VAPORIZATION PROCESS}

assume that no absorption takes place in the oil or water surrounding the shell material. Oil is not expected to vaporize since the vaporization temperature of hexadecane is $286^{\circ} \mathrm{C}$. Also, the core contents did not have an influence on the bubble response. The energy from the shell is transferred into the vapor bubble $\left(W_{p o l}\right)$, which consists exclusively of water vapor. Secondly, we have to take into account the thermal losses through the vapor/liquid interface $\left(W_{\text {loss }}\right)$. The vapor is considered to have a uniform temperature which is the vaporization temperature of water $T_{\text {vap }}$. The water far away from the bubble resides at room temperature, resulting in a temperature gradient and dissipation of energy from the vapor into the liquid phase. Finally the energy required to displace the vapor/liquid interface and to vaporize the water has to be calculated ( $\left.W_{\text {vap }}\right)$. The energy balance then reads:

$$
W_{\text {vap }}=W_{\text {pol }}-W_{\text {loss }} .
$$

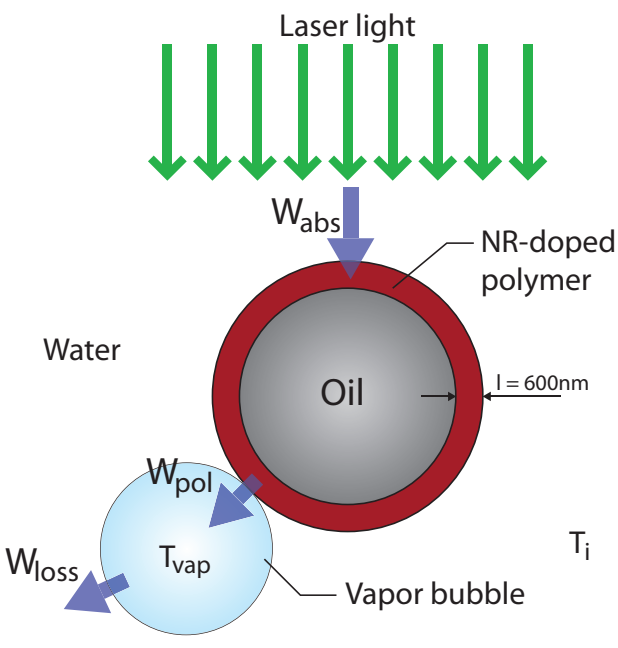

Figure 6.10: Schematic representation of the physical model of the vaporization process.

We can identify two separate time domains. First, the activation of the vaporization through absorption of the laser light and second, the dynamics of the formed vapor bubble. Both domains will now be discussed for the continuous and pulsed excitation. As a first approximation, the vapor bubble is assumed to be spherical, although the high-speed recordings in figure $\mathbf{6 . 4 3}$ indicate that the interface is not perfectly round. 


\subsubsection{Activation}

When the laser light heats up the polymer, the shell will reach its melting temperature $T_{\text {melt }}\left(145^{\circ} \mathrm{C}\right)$ and subsequently break at a certain point on the shell. The time it takes to reach this point can be obtained by solving the static solution for the thermal diffusion equation:

$$
I \mu_{a b s}\left(1-q_{e}\right)\left[\frac{R_{e}^{3}-R_{i}^{3}}{3 \lambda_{w} R_{e}}+\frac{1}{\lambda_{p}}\left(\frac{R_{e}^{2}}{6}+\frac{R_{i}^{3}}{3 R_{e}}-\frac{R_{i}^{2}}{2}\right)\right]=T_{m e l t}-T_{i}
$$

where $\mu_{a b s}$ is the absorption coefficient of the NR-doped polymer, $\left(q_{e}\right)$ is the quantum yield of NR, $I$ is the laser intensity, $R_{e}$ and $R_{i}$ are the external and internal radii of the shell, respectively, $\lambda_{p}$ and $\lambda_{w}$ are the thermal conductivity of the polymer and the water, respectively. The quantum yield $\left(q_{e}\right)$ of NR was determined in previous studies [112] and was taken to be 0.7 . The absorption coefficient of the NR-doped polymer was measured and found to be $143 \mathrm{~cm}^{-1}$.

When the properties of the capsules are put into the equation an activation threshold intensity of $0.9 \mathrm{MW} / \mathrm{cm}^{2}$ is found, corresponding well to the experimentally obtained value of $1.5 \mathrm{MW} / \mathrm{cm}^{2}$

By solving the dynamic thermal diffusion equation, the temperature profile inside the shell during the heating of the shell can be calculated assuming spherical symmetry and using the substitution $\tilde{T}=r T$ :

$$
D \frac{\partial^{2} \tilde{T}}{\partial r^{2}}+\frac{r P}{\rho c_{p}}=\frac{\partial \tilde{T}}{\partial t}
$$

with $P$ the thermal energy density inside the polymer. A numeric solution was found by performing a Fourier transformation, which was solved numerically using Matlab:

$$
F(\tilde{T})=\frac{P}{D \omega^{4}}\left(1-e^{-D \omega^{2} t}\right)\left(\left(1+j \omega R_{w}\right) e^{-j \omega R_{w}}-\left(1+j \omega R_{i}\right) e^{-j \omega R_{i}}\right) .
$$

The time evolution of the temperature profile $T(r, t)$ was calculated by taking the inverse Fourier transform of equation 6.5 and is plotted in figure 6.11 for the first few microseconds. When the center of the polymer shell has reached its melting temperature, the temperature of the polymer at the outer wall is just above the boiling temperature of water, whereas the temperature of the oil at the inner wall, is further above the boiling point. Therefore it is hypothesized that when the capsule breaks as the polymer is melted, the oil on the inside and fragments of the polymer shell come into contact with the water and cause the onset of vaporization.

In the case of pulsed laser irradiation, the heat absorption is instantaneous. The specific diffusion length within the polymer for the duration of the laser 


\subsection{MODELING OF THE VAPORIZATION PROCESS}
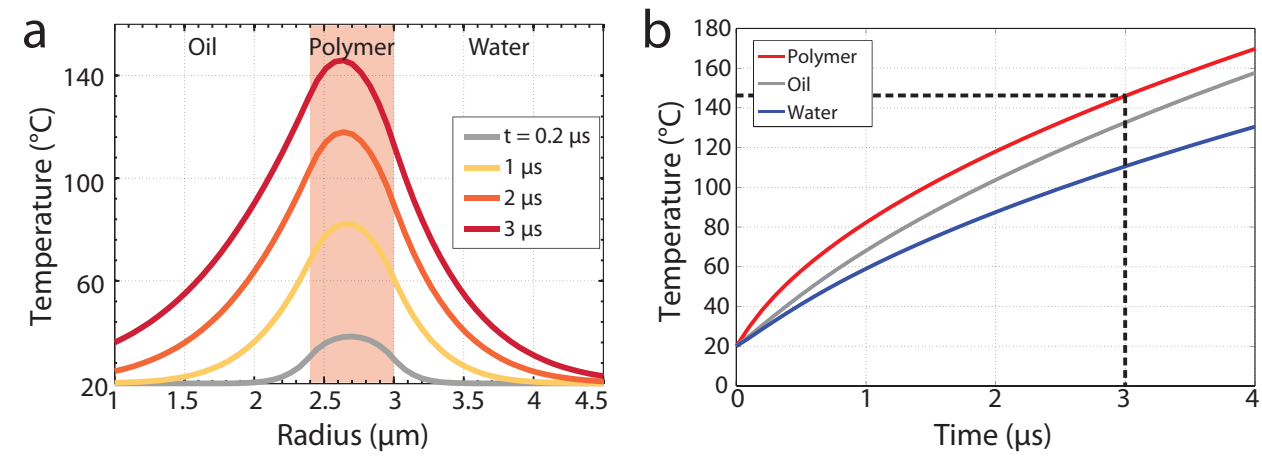

Figure 6.11: a) Temperature profile of the capsule shell during continuous laser irradiation as a function of the radius. b) Temperature inside the polymer shell and on the inner and outer wall of the shell as a function of time. At $t=3 \mu$ s the polymer reaches its melting temperature.

pulse $(7 \mathrm{~ns})$ was calculated to be $30 \mathrm{~nm}$, assuring thermal confinement of the absorbed laser energy within the shell material. Since the laser pulse length is in the same order of the excited state lifetime of the NR molecules [113] and the high laser fluence probably causes saturation [114], the measured absorption coefficient is not valid in case of the pulsed excitation. Therefore the initial temperature of the polymer shell is taken as an input parameter to model the dynamics of the vapor.

\subsubsection{Vapor bubble dynamics}

The interaction of the continuous laser irradiation with the polymer shell is complex, since the oscillations of the vapor bubble influence the amount of light reaching the shell fragments and the displacement of the shell fragments is on the order of the size of the laser spot. Therefore the vapor bubble dynamics have not been resolved yet for CW excitation.

Focussing of the laser beam led to a laser spot with a width comparable to the typical diameter of the capsules. Therefore, during the vapor bubble oscillations, the doped shell material that surrounds the bubble continuously moves in and out of the laser focus, as depicted schematically in figure 6.12 . When the vapor bubble is small, the shell pieces are irradiated by the laser, leading to heating and vaporization. When the bubble grows outside the laser spot, the shell pieces are no longer heated by the laser and the vapor bubble then looses its energy by thermal diffusion through the vapor/liquid interface. For a laser spot of $10 \mu \mathrm{m}$ in radius, the heating and diffusion balance each other and 
a stable vapor bubble of the same size can be formed, as shown in figure 6.8 . This equilibrium radius was matched by the average radius of oscillating vapor bubbles in the experiments.

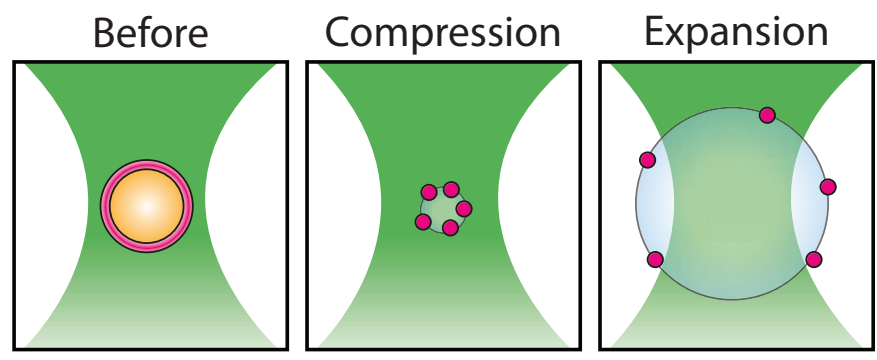

Figure 6.12: a) Schematic representation of the vapor bubble dynamics during continuous laser irradiation. When the vapor bubble is compressed, the shell fragments are located within the laser spot, they absorb laser energy and transfer the energy into the vapor. When the bubble expands, the shell fragments move away from the laser spot, reducing the energy transfer into the vapor.

Following the instantaneous heat absorption of the pulsed laser light by the polymer shell, the energy transferred from the polymer shell $W_{p o l}$ drives the dynamics of the vapor bubble. The shell itself is modeled by a rectangular slab with the same thickness $l$ and volume as the capsule shell in order to have the correct diffusion time and correct amount of energy stored inside the polymer. The temperature profile inside the polymer can be calculated using Fourier series to solve the diffusion equation assuming a constant temperature $T_{0}$ outside the wall:

$$
\begin{gathered}
T(x, t)=4 \frac{T_{0}}{\pi} \sum_{n=0}^{\infty} \frac{1}{2 n+1} \sin \left((2 n+1) \pi \frac{x}{l}\right) \alpha_{n}, \\
\alpha_{n}=e^{\left(-\frac{D_{p}(2 n+1)^{2} \pi^{2} t}{l^{2}}\right)},
\end{gathered}
$$

where $T$ is the temperature as a function of time and space, $D_{p}$ is the thermal diffusivity of the polymer, $l$ is the wall thickness and $x$ is the depth in the polymer wall. The gradient of the temperature at the side of the wall gives the power sent by the polymer and an integration over time results in the time-resolved energy $W_{p o l}$ sent:

$$
W_{p o l}=8 \frac{l \lambda_{p} S_{p} T_{0}}{D_{p} \pi^{2}} \sum_{n=0}^{\infty}\left(1-\frac{\alpha_{n}}{(2 n+1)^{2}}\right),
$$




\subsection{MODELING OF THE VAPORIZATION PROCESS}

where $\lambda_{p}$ is the thermal conductivity of the polymer and $S_{p}$ the surface area of the wall. We consider a constant vaporization temperature throughout the vapor bubble. The temperature profile in the surrounding liquid then reads:

$$
T(r, t)=\left(T_{\text {vap }}-T_{i}\right)\left(\frac{R}{r}-\frac{R}{r \sqrt{\pi D_{w} t}}\right) \int_{0}^{r-R} e^{-\frac{s^{2}}{4 D_{w t}}} d s,
$$

where $T_{\text {vap }}$ is the vaporization temperature of water, $T_{i}$ is the ambient temperature of the water far away from the capsules as well as the initial temperature $T(r, 0), R$ is the radius of the bubble, $D_{w}$ is the diffusivity of water and $r$ is the distance from the center of the bubble. Again the temperature gradient results in diffusion of thermal energy, giving rise to a energy loss from the vapor into the liquid $W_{\text {loss }}$ :

$$
\begin{gathered}
W_{\text {loss }}=\theta \int_{0}^{t} \varepsilon R^{2}\left(\frac{1}{R}+\frac{1}{\sqrt{\pi D_{w} t}}\right) d t, \\
\varepsilon=4 \pi \lambda_{e}\left(T_{\text {vap }}-T_{i}\right),
\end{gathered}
$$

with the thermal conductivity of water $\lambda_{e}$. A fitting parameter $\theta$ is introduced to correct for bubble size variations on the temperature gradient.

The dynamics of the vapor bubble interface is described by the RayleighPlesset equation. The amount of vaporized water has little influence on the motion the interface, the compressibility of the water is considered negligible with regard to the bubble size and the velocity of the interface during the growth phase is small compared to the speed of sound, resulting in a simplified equation:

$$
\rho_{w}\left(R \ddot{R}+\frac{3}{2} \dot{R}^{2}\right)=P_{\text {gas }}-P_{0}-\frac{2 \sigma}{R}-4 \mu \frac{\dot{R}}{R},
$$

where $P_{\text {gas }}$ is the gas pressure, $\sigma$ is the surface tension of water, $\mu$ is the viscosity of water, $P_{0}$ is the ambient pressure and $\rho_{w}$ is the mass density of water. The pressure can be related to the energy through the ideal gas law and the enthalpy. Combining the three equations results in the following model:

$$
\begin{gathered}
\rho_{w}\left(R \ddot{R}+\frac{3}{2} \dot{R}^{2}\right)+\frac{2 \sigma}{R}+4 \mu \frac{\dot{R}}{R}+P_{0}=\beta\left(W_{\text {pol }}-W_{\text {loss }}\right), \\
\beta=\frac{3 R_{g} T_{\text {vap }}}{4 \pi R^{3} M_{\text {mol }}\left(h_{\text {vap }}+c_{p, w}\left(T_{\text {vap }}-T_{i}\right)\right)},
\end{gathered}
$$

where $h_{v a p}$ is the vaporization enthalpy of water, $c_{p, w}$ is the specific heat capacity of water and $R_{g}$ the gas constant. The fitting parameter $\theta$ was fixed to $1 / 40$. An 
extra damping factor of 13 was added to the viscosity of water. This damping factor was determined from the rebound of the vapor collapse observed for gasfilled capsules. This added damping may be caused by the presence of polymer at the vapor/liquid interface.

The above model predicts an initial temperature of the shell material far above the melting point of the polymer when high laser fluences are applied, explaining the explosive-like growth of the bubble. A comparison of the model and the experimentally obtained radius-time curves is plotted in figure 6.13. The overall behavior is captured by the model for both laser fluences. The deviation found after the primary collapse can be explained by the limitation of the optical recordings, as the measured radius consists of the radius of the vapor bubble plus the radius of the capsule fragments.
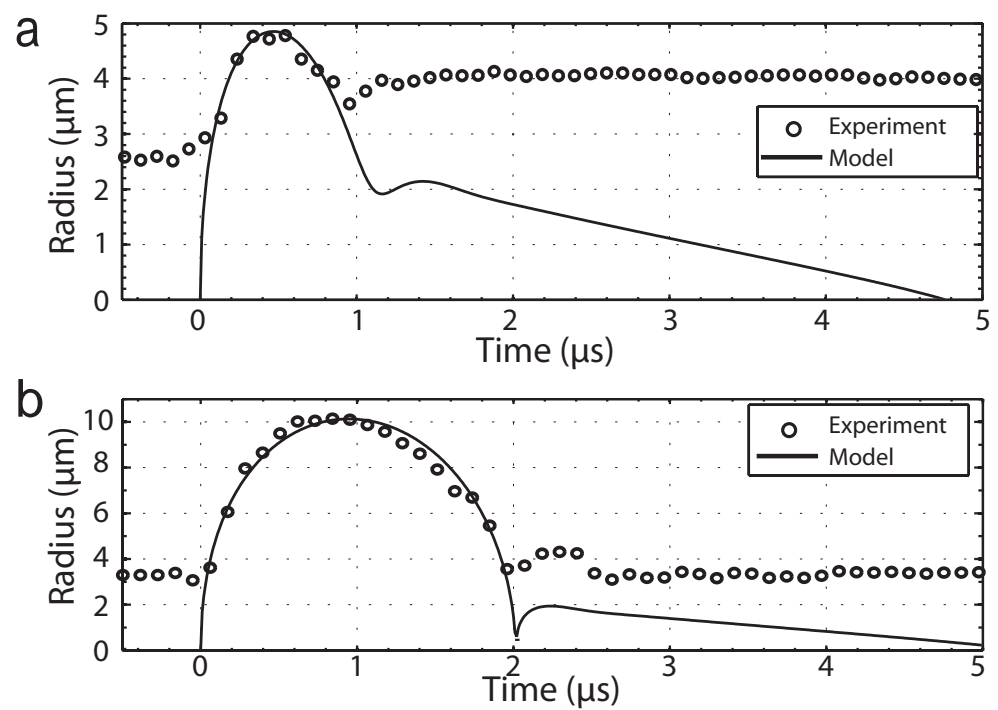

Figure 6.13: Comparison of the experimental and modeled radius-time curve for a laser fluence of a) $72 \mathrm{~mJ} / \mathrm{cm}^{2}$ and b) $1.44 \mathrm{~J} / \mathrm{cm}^{2}$.

\subsection{Conclusions and discussion}

The developed polymeric microcapsules are susceptible to ultrasound [23] and therefore have great potential as a multimodal contrast agent for photoacoustic imaging and ultrasound imaging. The design of the capsules allows for the encapsulation of a payload in the core, making them versatile therapeutic agents both for ultrasound-triggered drug delivery and laser-triggered drug de- 


\subsection{CONCLUSIONS AND DISCUSSION}

livery. However, the efficacy of drug delivery following acoustic or photoacoustic activation has not yet been investigated.

The acoustic signals generated by the vaporization from single microcapsules shows an excellent signal strength compared to photoacoustic contrast agent nanospheres. Modeling of the physical mechanisms involved in the vaporization process was in agreement with the experimentally observed phenomena, giving insight into various ways to improve the efficiency of the capsules and to further reduce the threshold for activation.

Due to their susceptibility to continuous laser irradiation the microcapsules may also serve as contrast agents for frequency domain photoacoustic imaging. The results shown here are based on a limited spot size that acted as an amplitude modulation of the laser excitation. Time-modulation of the laser light can be used to obtain a similar effect and experiments in this direction are underway.

To obtain a fully biocompatible photoacoustic microcapsule, Nile Red will have to be replaced by a different absorbing dye or particle. The laser fluences and intensities used in this study were close to the MPE for medical applications. However, by shifting towards the infrared region of the spectrum the penetration depth will be enhanced and higher laser fluences can be used. Replacing Nile Red with an absorbing dye that has little to no fluorescence emission, will increase the efficiency of the laser energy transfer into vaporization. Finally, a size reduction is desirable. For enhanced tumor imaging, capsules with a size down to a nanometer scale are required. 


\section{7 \\ Biodegradable polymeric microcapsules for selective ultrasound-triggered drug release ${ }^{1}$}

A series of hollow biodegradable polymeric microcapsules were prepared, of which their susceptibility to ultrasound was used for triggered release. Highspeed imaging of the ultrasound experiments showed a strong correlation between the acoustic pressure needed to activate these microcapsules and their shell thickness to diameter ratio. Based on this information a selective triggering of capsules with two different shell thickness to diameter ratios was successfully performed. The capsules were mixed in a single system and were activated independently from each other by a differentiation in acoustic pressure levels. This application is of great interest in the field of two-step drug delivery, since this system allows for localized multiple drug releases in a selective fashion.

\footnotetext{
${ }^{1}$ Published as: Dennis Lensen, Erik Gelderblom, Dennis Vriezema, Philippe Marmottant, Nico Verdonschot, Michel Versluis, Nico de Jong and Jan van Hest, Biodegradable polymeric microcapsules for selective ultrasound-triggered drug release, Soft Matter 7, pp. 5417-5422 (2011).
} 


\subsection{INTRODUCTION}

\subsection{Introduction}

Diagnostic ultrasound imaging is nowadays routinely performed with ultrasound contrast agents (UCAs). Typically, UCAs are microcapsules with a diameter between 1 and $10 \mu \mathrm{m}$, which are comprised of a gas-filled interior. The microcapsule shell can be composed of a broad variety of molecules, including lipids, proteins, polysaccharides or synthetic polymers [6-8, 59, 115]. UCAs scatter ultrasound efficiently and they also respond to low energy ultrasound by emitting harmonic frequencies, resulting in contrast enhancement with respect to the surrounding tissue. Air-filled microbubbles are not very practical as contrast agents, since the air dissolves very rapidly in the blood stream and the bubbles are lost from the circulation before the ultrasound examination can be completed. Fluorinated gases combined with a stabilizing shell are therefore commonly used to stabilize the microbubbles for a sufficiently long time; around 5-10 minutes is possible [59, 116]. Alternatively, air-filled microbubbles with fluorinated polymer shells have also been used for this purpose [23, 41, 110].

UCAs are generally classified as soft-shelled or as hard-shelled agents. Softshelled agents are microbubbles of which the shell is composed of a lipid monolayer with a thickness of 2-3 nm. They undergo volume expansions and contractions that generate an acoustic signal in the far-field of which the non-linear subharmonic or harmonic components give their specific contrast enhancement for medical ultrasound imaging. [5, 18, 20] A drawback of these soft-shelled agents is that upon expansion and contraction of the flexible lipid membrane at elevated pressures, submicron-sized lipid aggregates such as micelles and liposomes are shed from the microbubble, thereby destroying the UCAs. [117]

The current theoretical models for soft-shelled microbubble triggering using ultrasound incorporate the response of the shell by exploring small amplitude vibration [18, 118-121]. However, for discriminating a contrast agent from the surrounding tissue, a non-linear response of the large amplitude vibration regime is of importance, caused by either buckling [122] or destruction of the contrast agent [123, 124]. In buckling, a monolayer of lipids shows 'compression-only' behavior [2, 19]. Here, only the compression is significant, while no expansion occurs, since these lipid monolayers do not undergo in-plane compression. The bending modulus of the monolayer is very small, so it is energetically more favorable to bend the membrane than to compress it.

Hard-shelled microbubbles have a typical shell thickness in the range of 20$100 \mathrm{~nm}$ and are usually prepared from polymers. They hardly show volume expansions at low acoustic pressure due to the increased damping contribution of the polymer shell and remain intact until a certain pressure threshold is reached. Above this threshold their shell ruptures and the gas core escapes [125]. It is 
believed that ultrasound echoes are most likely generated only after shell disruption and gas release. However, some hard-shelled microbubbles do generate acoustic signals without losing gas [23]. These bubbles often indent through a buckling instability, which is a way to conserve their surface area and allow for a change in the volume. By using fluorinated polymers for the microbubble shell, the water intrusion through pores in the shell is very much reduced due to the hydrophobic surface properties of these polymers [23, 41, 126].

Recently Marmottant et al. have developed a new theory describing the behavior of hard-shelled microbubbles when undergoing buckling or rupturing using ultrasonic pressure [I2]]. Such microbubbles can more easily sustain inplane compression in contrast to the soft-shelled microbubbles, thus allowing for stabilization against dissolution of the gas inside. This, however, holds for limited compressions as these microbubbles also undergo buckling if the compressive constraint is large enough. They also compared this theoretical study with the experimental results of Bouakaz et al. obtained earlier, in which the contrast agent PB127 (Point Biomedical) was used [123].

Ultrasound-triggered drug release has attracted increasing interest, since the release trigger can be applied locally [7, 115, 128]. Böhmer et al. have recently shown that polymeric PLLA capsules with different shell thicknesses can be triggered both in vitro and in vivo, although using different ultrasonic pressures [128]. They incorporated a model drug, Evans blue, in liposomes and polymeric capsules with different shell thicknesses and triggered them in a gel using high pressures. By comparing the destruction zone of the capsules as a function of the ultrasonic pressure, they observed that a thicker shell required a higher ultrasonic pressure. They varied the ultrasonic pressure in rather big steps of $1 \mathrm{MPa}$. At higher pressures hard-shelled capsules were destroyed with dramatic changes in the gas volume [23, 123, 125]. Although this leads to a loss of US properties after breakage, it also opens up the possibility of using UCAs as drug delivery vehicles, since the capsule content is efficiently released. Furthermore, by applying both low and high US pressure, UCA-based drug delivery vehicles can be used for monitoring and release, respectively, which substantially improves therapeutic treatment. A next step forward would be realized if UCA drug delivery vehicles could be employed in selective drug release. This could be achieved if a mixture of microcapsules is used which releases its encapsulated drugs at different ultrasound parameters, such as pressure, pulse repetition frequency or pulse length.

In this paper we describe in detail the ultrasonic pressure needed to trigger a series of biodegradable polymeric microcapsules with adjustable sensitivity toward ultrasound. Tuning of the shell thickness of a capsule made it possible to selectively trigger capsules using diagnostic ultrasound at a mechanical in- 


\subsection{EXPERIMENTAL}

dex of 1.2, which was well below the maximum mechanical index [129] of 1.9 as regulated by the FDA [130]. Also a strong correlation was found between the activation pressure of the ultrasound, the pressure at which a capsule shell buckles or ruptures, and the shell thickness of similar sized capsules. These results were in perfect agreement with the theoretical model of Marmottant et al. for hard-shelled microcapsules. Furthermore, selective triggering of microcapsules in a single system having different shell thicknesses was proven, which is a promising step toward more controlled drug delivery applications.

\subsection{Experimental}

\subsubsection{Materials}

L-Lactide, poly(vinyl alcohol) (PVA, $M_{\mathrm{w}}$ 9-10 kDa, 80\% hydrolyzed), methoxypoly(ethylene glycol) (PEG, $\left.M_{\mathrm{w}}=3350\right)$, tetrahydrofuran (THF) and stannous(II) octanoate $\left(\mathrm{Sn}(\mathrm{Oct})_{2}\right)$ were purchased from Sigma-Aldrich (Schnelldorf, Germany). Decane was purchased from Fluka (Schnelldorf, Germany). 2,2,3,3,4,4,5,5,6,6,7,7,8,8,8-pentadecafluoro-1-octanol (PFO) was purchased from ABCR (Karlsruhe, Germany). Methanol was purchased from Baker (Griesheim, Germany). Dichloromethane was distilled over $\mathrm{CaCl}_{2}$ and toluene was distilled over $\mathrm{Na}$ /benzophenone. All other chemicals were used as received.

\section{Equipment}

${ }^{1} \mathrm{H}$ NMR spectra were recorded on a Varian $400 \mathrm{MHz}$ at room temperature and with $\mathrm{CDCl}_{3}$ as a solvent containing tetramethylsilane as the internal standard.

Molecular weights of the polymers were measured using size exclusion chromatography (SEC) on a Shimadzu system equipped with a guard column and a $\mathrm{PL}$ gel $5 \mu \mathrm{m}$ mixed $\mathrm{D}$ column (Polymer Laboratories) with a differential refractive index detector, using THF as an eluent at $1 \mathrm{~mL} / \mathrm{min}$ and $T=35^{\circ} \mathrm{C}$. Polystyrene standards in the range of 580 to $377400 \mathrm{~g} / \mathrm{mol}$ (Polymer Laboratories) were used to calibrate the SEC.

Differential Scanning Calorimetry (DSC) measurements were performed on a Perkin Elmer Pyris Diamond DSC, equipped with a Perkin Elmer Intracooler 2P. Samples were prepared in an aluminium cup with less than $1 \mathrm{mg}$ of sample. Empty aluminium cups were used as a reference. All samples were measured with heating rates of $5{ }^{\circ} \mathrm{C} /$ minute.

Cryo-SEM was performed on a JEOL JSM-6330F electron microscope. All samples were sputtered with a layer of $\mathrm{Pd} / \mathrm{Au}$. 
Samples used for lyophilization were rapidly frozen in liquid nitrogen. Lyophilization was performed with an Ilshin FD 8515 with a condenser temperature of $-92^{\circ} \mathrm{C}$ at $5 \mathrm{~m}$ Torr for at least 24 hours.

\subsubsection{Synthesis of PFO-PLLA}

Poly(L-lactic acid) terminated by PFO was synthesized according to Lee et al [I26]. PFO $(1.02 \mathrm{~g}, 2.55 \mathrm{mmol})$ and L-lactide $(6.61 \mathrm{~g}, 0.46 \mathrm{~mol})$ were suspended in toluene $(50 \mathrm{~mL})$ under an argon atmosphere, while stirring. After 5 minutes $\mathrm{Sn}(\mathrm{Oct})_{2}(10 \mathrm{mg}, 26 \mathrm{mmol})$, dissolved in toluene $(1 \mathrm{~mL})$, was added to the suspension and the mixture was heated to $130^{\circ} \mathrm{C}$ for 18 hours. The mixture was allowed to cool to room temperature and evaporated to dryness. The resulting white polymer was dissolved in a minimal amount of dichloromethane and precipitated in methanol. The precipitate was filtered off and was purified once more by the procedure described above. The precipitate was dried under high vacuum for 24 hours to yield a white solid $(6.30 \mathrm{~g}$, yield: $73 \%)$. The ${ }^{1} \mathrm{H}$ NMR spectrum of the resulting polymer was similar as the polymer described in the literature [41].

Molecular weight determination: NMR: $M_{\mathrm{n}}=3395 \mathrm{~g} / \mathrm{mol}$, GPC: $M_{\mathrm{n}}=6233 \mathrm{~g} / \mathrm{mol}, M_{\mathrm{w}}=8583 \mathrm{~g} / \mathrm{mol}, \mathrm{DPI}=1.38$.

\subsubsection{Procedure for preparing PFO-PLLA microcapsules}

PFO-PLLA was weighed and dissolved in dichloromethane $(2.0 \mathrm{~g})$ and to this decane $(0.76 \mathrm{~g})$ was added. This solution was added slowly to a solution of PVA in water $(1 \mathrm{wt} \%, 15 \mathrm{~mL})$ stirred at $10000 \mathrm{rpm}$ using an ultraturrax. The formed emulsion was stirred for an additional 5 minutes and then transferred to a beaker containing PVA in water $(1 \mathrm{wt} \%, 40 \mathrm{~mL})$ and stirred for 24 hours using a magnetic stirring bar to allow the dichloromethane to evaporate. The dispersion was centrifuged at $3000 \mathrm{rpm}(605 \mathrm{~g})$ for 10 minutes, followed by isolation of the top layer of floating capsules. The capsules were redispersed in a PEG solution (5 wt\%) and centrifuged twice more at $3000 \mathrm{rpm}(605 \mathrm{~g})$, each time redispersing the floating capsules. Finally the floating capsules were isolated, redispersed in a minimal amount of PEG solution and lyophilized to remove the decane from the lumen. These gas-filled capsules could be stored for more than 1 year, while remaining ultrasonically active in solution.

Fluorescent PFO-PLLA capsules were prepared identical to the procedure described above, only Nile Red (1 mg) was added to the polymer solution. 


\subsection{RESULTS AND DISCUSSION}

\subsubsection{Ultrasound measurements}

Microcapsules were dispersed in double distilled water (dd water, $R=18.2 \mathrm{M} \Omega$ ) and injected in an Opticell ${ }^{\mathrm{TM}}$. This cell was placed under water and the microcapsules were measured by a setup described by Overvelde et al [37]. The capsules were insonified using a $1.0 \mathrm{MHz}$ single element transducer (Precision Acoustics Ltd) transmitting 16-cycle-sine wave bursts, with pressures between 50 and $1200 \mathrm{kPa}(\mathrm{MI}=0.05-1.2)$, as verified with a calibrated $0.2 \mathrm{~mm}$ PVDF needle hydrophone (Precision Acoustics Ltd). Images of insonified microcapsules were recorded using a $60 \times$ water-immersed objective and $2 \times$ magnifier, and recorded in six sequences of 128 image frames at a frame rate near 5 million frames per second using the Brandaris 128 ultra-high-speed camera system [15]. Images were processed using custom-designed software written in Matlab (Mathworks).

For the selective ultrasound measurement an identical ultrasound setup was used as described above. The capsules were insonified using a $1.0 \mathrm{MHz}$ single element transducer (C302; Panametrics-NDT; Olympus-NDT) transmitting 16cycle-sine wave bursts, with pressures between 50 and $1200 \mathrm{kPa}(\mathrm{MI}=0.05-$ 1.2), as verified with a calibrated $1.0 \mathrm{~mm}$ PVDF needle hydrophone (Precision Acoustics Ltd). The PFO-PLLA capsules with Nile Red were visualized using an excitation wave length of $550 \mathrm{~nm}$ and emission snapshots were recorded at $580 \mathrm{~nm}$. Overlay images of bright-field and fluorescent snapshots of insonified microcapsules were recorded using a $40 \times$ water-immersed objective and $2 \times$ magnifier using custom-designed software written in Matlab.

\subsubsection{Cryo-SEM measurements of PFO-PLLA capsules}

Lyophilized PFO-PLLA capsules were dispersed in dd water and manually shaken in an eppendorf tube. The dispersion was centrifuged at $3000 \mathrm{rpm}(605 \mathrm{~g})$ and the supernatant was taken out using a syringe fitted with a needle. The capsules were redispersed in dd water and the procedure was performed two more times. Finally the capsules were dispersed in dd water and rapidly frozen in liquid nitrogen.

\subsection{Results and discussion}

For the preparation of ultrasound responsive microcapsules with different shell thicknesses, an emulsionevaporation technique was employed [I3I]. Here, an oil-in-water emulsion was used, in which the oil phase consisted of a mixture of the shell-forming polymer, in this case perfluoroctanolpoly(lactic acid) (PFO-PLLA) [126], a volatile solvent, dichloromethane, and a non-volatile non- 
solvent, decane. As the dichloromethane started to evaporate small polymerrich droplets were formed by phase separation within the emulsion droplets. These polymer-rich droplets migrated to the interface and engulfed under the right conditions the original droplet. Further removal of the volatile solvent resulted in more polymer precipitate which migrated to the shell, thus forming coreshell microcapsules. By using different initial concentrations of polymer in the organic phase and by maintaining the same mechanical energy for droplet formation, four different capsules were prepared with increasing shell thickness, see table $[$.$] .$

In order to obtain hollow biodegradable microcapsules, decane was removed from the core using lyophilization. No deformations of the capsules were observed before and after lyophilization as was presented in a previous study [132]. The removal of decane from the core of these capsules was confirmed by DSC measurements, as it was observed that no melting peak for decane was found at $-28^{\circ} \mathrm{C}$ after freeze drying for all four types of capsules.

By using cryo-SEM it was also possible to determine the capsule diameters and their shell thicknesses (Table $7 \mathbb{7}$ ) as depicted in figure 7.7 . Furthermore, the dispersities of the capsule sizes $(5.3 \mu \mathrm{m} \pm 2.3 \mu \mathrm{m})$ have also been determined from SEM measurements.

Table 7.1: Overview of the properties of PFO-PLLA microcapsules prepared using an emulsion-evaporation technique with different polymer concentrations

\begin{tabular}{|c|c|c|c|c|c|}
\hline Capsule & $\begin{array}{l}\text { Polymer } \\
\text { mass }^{1} \\
(\mathrm{mg})\end{array}$ & $\begin{array}{l}\text { Calculated } \\
\text { shell } \\
\text { thickness } \\
(\mathrm{nm})\end{array}$ & $\begin{array}{l}\text { Measured } \\
\text { shell } \\
\text { thickness }{ }^{2} \\
(\mathrm{~nm})\end{array}$ & $\begin{array}{l}\text { Measured } \\
\text { shell } \\
\text { diameter }{ }^{2} \\
(\mu \mathrm{m})\end{array}$ & $\begin{array}{l}\text { Shell thickness } \\
\text { to diameter } \\
\text { ratio }\left(10^{-3}\right)\end{array}$ \\
\hline 1 & 100 & 43 & $65 \pm 2$ & $3.54 \pm 0.07$ & $18 \pm 0.5$ \\
\hline 2 & 200 & 97 & $121 \pm 3$ & $4.16 \pm 0.10$ & $29 \pm 1.5$ \\
\hline 3 & 300 & 144 & $196 \pm 4$ & $4.29 \pm 0.09$ & $46 \pm 1.5$ \\
\hline 4 & 500 & 137 & $158 \pm 6$ & $2.67 \pm 0.11$ & $59 \pm 4.3$ \\
\hline
\end{tabular}

${ }^{1}$ All polymers were dissolved in $1.5 \mathrm{~mL}$ dichloromethane and $1.0 \mathrm{~mL}$ decane. The emulsion was prepared by stirring at $10000 \mathrm{rpm}$ using an ultraturrax.

2 Average sizes from ten measurements obtained from cryo-SEM studies.

From figure $\mathrm{R} . \mathrm{l} b$ it is observed that the ratio between the shell thickness and the diameter shows a gradual increase upon increasing the polymer mass, which demonstrates that there is good control over the desired shell thickness. However, there is a slight deviation of the ratio shell thickness to diameter, since the thickness of the shell is not completely homogeneous. Furthermore, as expected, larger capsules have thicker shells as a result of a larger initial 


\subsection{RESULTS AND DISCUSSION}

droplet size, since more decane in the core will result in more polymer ending up in the shell.

Listed in table $[\mathbb{T}$ are the calculated shell thicknesses for these capsules. The calculated shell thicknesses were slightly lower than the measured values, which can be partly explained by the fact that the exact capsule radius was difficult to estimate from cryo-SEM, since the capsules were not fully exposed from the ice. Moreover, the calculation assumes ideal mixing conditions of the decane and the polymer solution and that all polymer material ends up in a microcapsule. This, however, may not have been achieved during microcapsule preparation and may have therefore led to an overestimation of the shell thickness. In order to investigate the effect of the ratio of the shell thickness to the capsule size on the response to ultrasound pressure, the capsule dynamics were studied optically using the Brandaris 128 ultra-high-speed camera during insonification of the capsules [15]. The pressure pulse that was applied to the microcapsules during ultrasound treatment is depicted in figure $7.2 \mathrm{~b}$. The Brandaris 128 camera was operated at a frame rate of 5 million frames per second (Mfps), thus allowing for a highly detailed insight in the behavior of the microcapsules during the applied pressure wave.
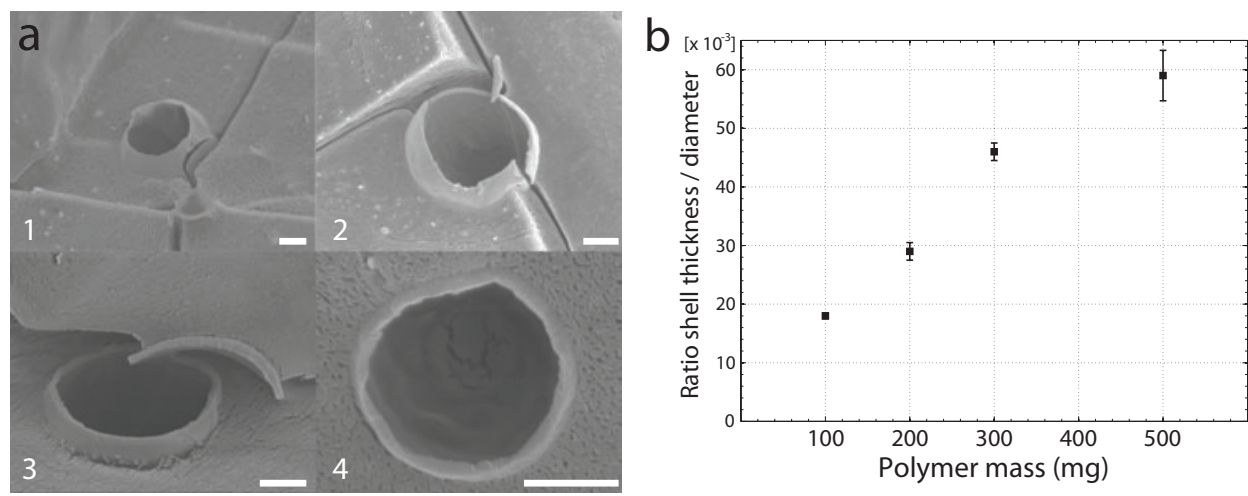

Figure 7.1: a) Scanning electron micrographs of PFOPLLA microcapsules 1-4 from Table [.] with different shell thicknesses. Scale bars represent $1 \mu \mathrm{m}$. b) Plot of the polymer mass versus the ratio of shell thickness to diameter.

At low acoustic pressures (50-250 kPa) no capsules were disrupted. However, the capsules with the thinnest shell (entry 1 in table 7.7 ) showed buckling behavior at an acoustic pressure of $250 \mathrm{kPa}$ as shown in figure 7.2la. Here, the capsules expansion and contraction correspond to the pressure at different times as indicated in figure $7.2 \mathrm{~b}$.

By increasing the acoustic pressure by $40 \%$ to $350 \mathrm{kPa}$, capsule 1 continued 


\section{BIODEGRADABLE MICROCAPSULES FOR DRUG RELEASE}

to buckle as depicted in figure Z.2a. However, above a certain acoustic pressure threshold, the shell yielded, resulting in rupture of the shell at its thinnest point and release of the gas content as depicted in figure $7.3(1 \mathrm{a}-\mathrm{e})$. The capsules with a higher shell thickness/diameter ratio did not show buckling behavior, apparently due to a shell thickness of these capsules that was too high to allow indentation. All capsules (1-4 from table 7.1 ) responded by rupturing when the acoustic pressure was sufficiently high.
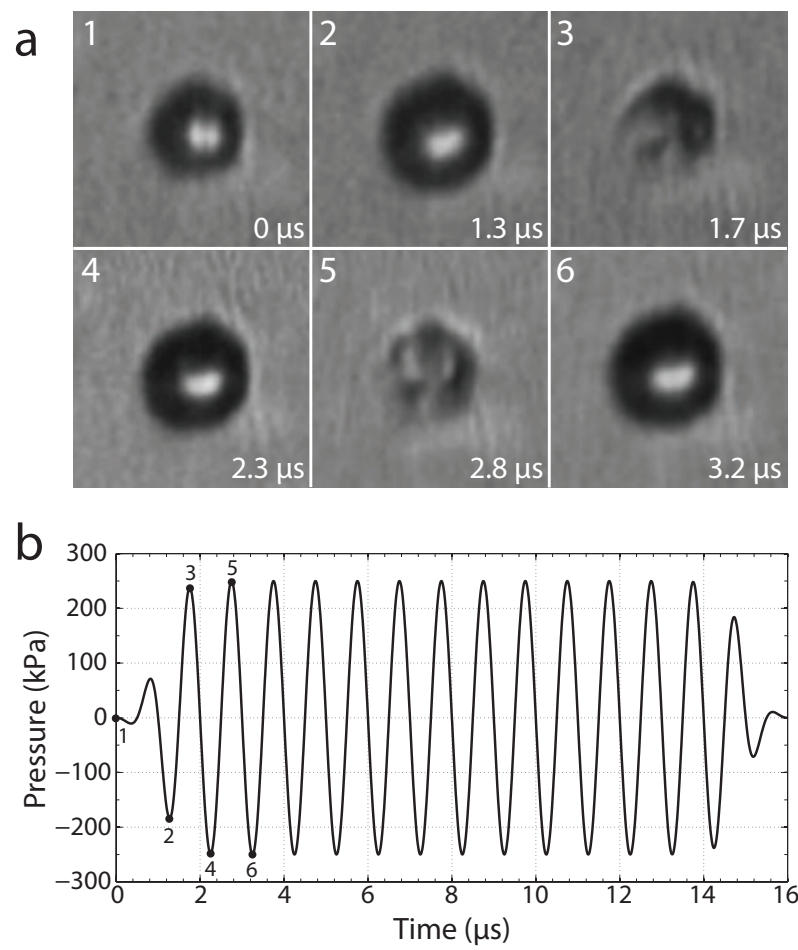

Figure 7.2: a) Buckling behavior of a thin-shelled polymer capsule (entry 1 in Table [.]) at $250 \mathrm{kPa}$. Numbers 1-6 correspond to the applied pressure as depicted in $\mathrm{b}$. b) Schematic representation of the sine wave of the applied ultrasound pressure.

From figure $[.3$ it can be observed that the release of the gas was more violent when the capsules had a thicker shell/diameter ratio. The capsules with higher ratios could withstand the expansion of the gas when insonified. When even higher acoustic pressures were used to trigger the capsules, the gas in the capsules expanded further, giving rise to a stronger, more impulsive gas release. The data points of the ratio of the calculated shell thickness and diameter of the capsules were plotted against the acoustic pressure of the ultrasound as 


\subsection{RESULTS AND DISCUSSION}

depicted in figure 7.4 .

It shows that the ratio of shell thickness to diameter of the capsules correlates strongly with the ultrasound pressure needed to disrupt the capsules. A buckling behavior was only observed for capsules with low shell/diameter ratios and at low ultrasound pressure. For the capsules with the larger shell thickness/diameter ratio this behavior was no longer observed and the capsules only responded by instant rupturing when the ultrasound pressure was above the release threshold.

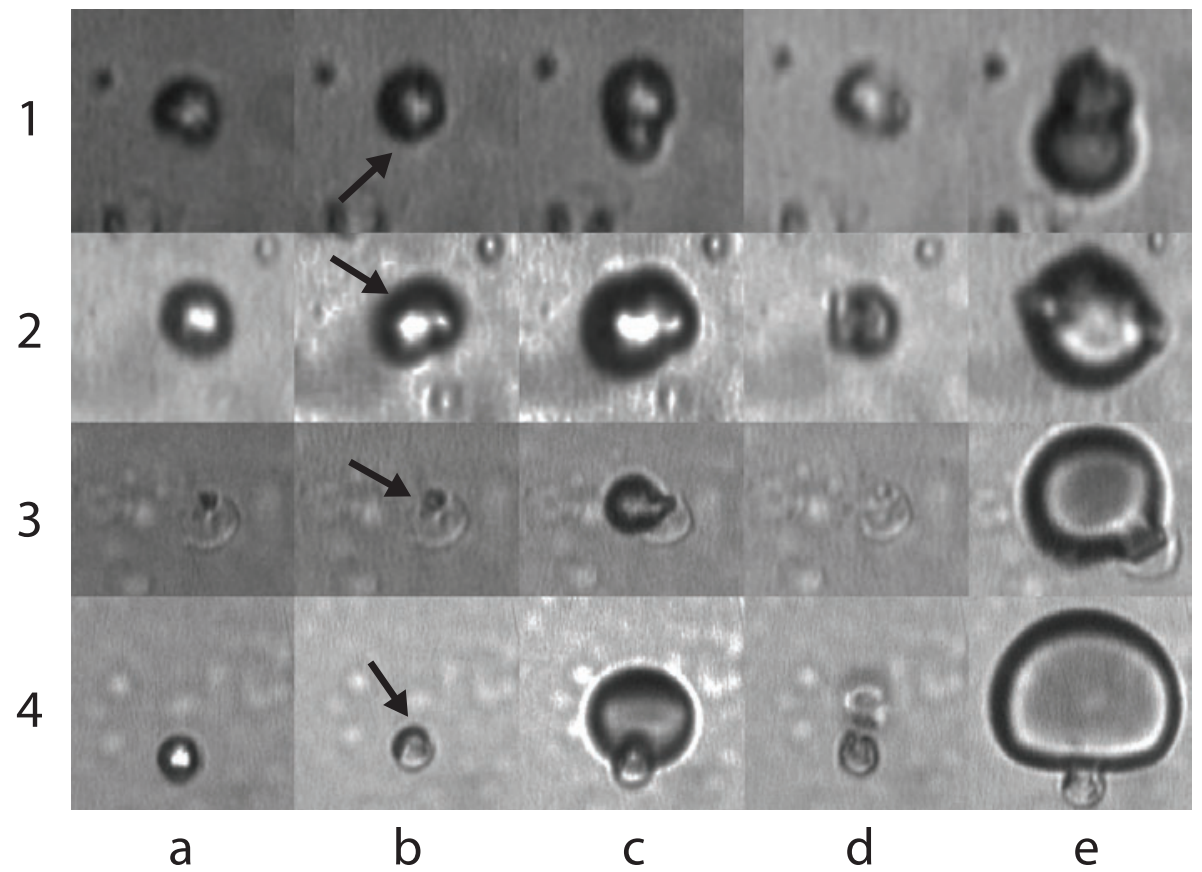

Figure 7.3: Rupture of the capsules 1-4 using ultrasound at high acoustic pressure. (a) Capsule just before rupture. (b) Shell of the capsule ruptures (arrow). (c) Gas escapes from the core of the capsule. (d) Contraction of the capsule, when a positive pressure is applied. (e) Second expansion of the gas and full rupture of the capsule, when maximal pressure is applied. Images are snapshots of movies recorded by the Brandaris 128 camera.

This behavior corresponds nicely with the recent theory of Marmottant et al. [127] where they predicted the rupture and buckling threshold for varying bubble radii. When the shell thickness to bubble diameter ratio is varied, the pressure for buckling onset is predicted to vary as $P_{\text {buckling }}=E(d / R)^{2}$, with $E$ the elastic Youngs modulus, $d$ the shell thickness and $R$ the bubble radius. The rupture is achieved when the shell material is elongated more than a critical 
elongation $e_{\max }$, and occurs at a pressure $P_{\text {buckling }}=3 E(d / R) e_{\max }$. The experimental data were compared with the theory of Marmottant et al. as shown in figure 7.4 [127]. They also stated that above a certain shell thickness/ diameter ratio, no buckling will be observed and the bubbles will only rupture while releasing their gas content, which is in perfect agreement with our observations.

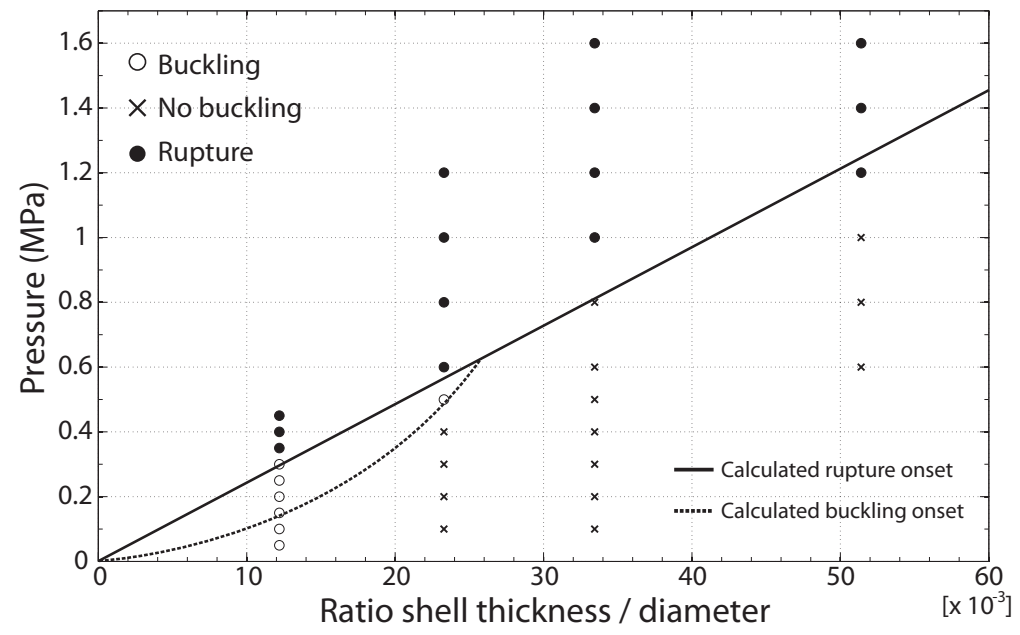

Figure 7.4: Plot of the ratio of capsule shell to capsule diameter against the acoustic pressure, together with the theoretical prediction for the onset of buckling and rupturing. The following parameters were used: a Youngs modulus of $E=225 \mathrm{MPa}$, and a critical elongation for rupture of $e_{\max }=1.8 \%$ [127]. Symbols in the graph represent the measured data points. The errors for the ratio shell thickness/diameter were calculated from the diameter of the capsules as observed in the high speed recordings and are respectively $12 \pm 1,23 \pm 2,33 \pm 3$ and $51 \pm 4.5$.

The good control over the ratio of shell thickness to diameter of these capsules, as well as their predictable behavior toward ultrasound pressure, makes these capsules ideal as selective drug delivery vehicles. In order to demonstrate this, two different capsules were mixed with markedly different ratios (capsules 1 and 4 in table 7.1 ). In order to distinguish between these capsules, the thinshelled capsules were loaded with the dye Nile Red, which was visualized using fluorescence microscopy. An overlay image of the fluorescence microscope image and the bright-field microscope image marks the location of the different capsules as can be seen in figure T.5la.

By first applying a low pressure $(400 \mathrm{kPa})$, the thin shelled capsules were activated as depicted in figure $7.5 \mathrm{~b}$. The wall of these capsules was disrupted allowing water to penetrate into the capsules. As a consequence the microcapsules sank and moved out of focus of the microscope. The larger capsule 


\subsection{CONCLUSIONS}
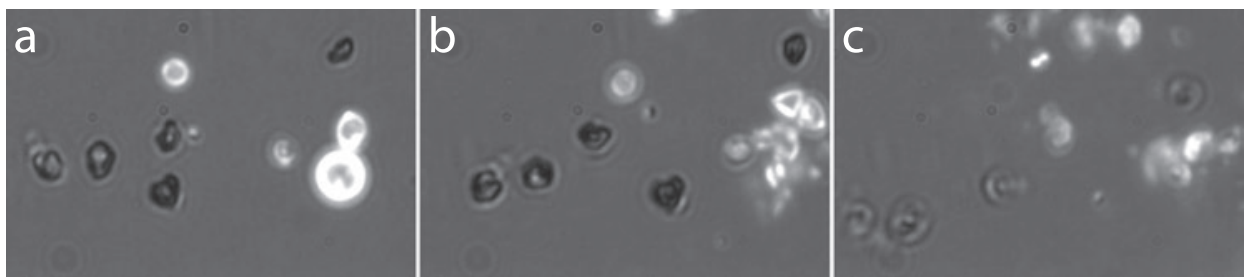

Figure 7.5: Overlay microscope images of mixed microcapsules having different ratios of shell thickness to diameter, triggered by ultrasound. a) Image of the microcapsules when no pressure was applied. b) Image of the microcapsules when $400 \mathrm{kPa}$ of pressure was applied. c) Image of the microcapsules when 1.2 MPa was applied.

showed a more violent disruption and displacement than the smaller ones, which is due to the bigger volume of gas present in this capsule. From this image it was observed that all the thinner shelled capsules with the same ratio of shell thickness/diameter were triggered. When applying a second pressure pulse of 1.2 $\mathrm{MPa}$, also the thicker shelled microcapsules were disrupted (figure $7.5 \mathrm{c}$ ). It can be clearly seen that the gas, which was entrapped in these capsules had escaped, while the shells of the capsules remained. This demonstrates that capsules with different shell thicknesses can be triggered independently from each other using different ultrasonic pressures, thereby releasing their contents.

\subsection{Conclusions}

In this paper we have shown the preparation of a series of microcapsules with an adjustable shell thickness to diameter ratio. By controlling the ultrasound parameters we have demonstrated with high speed imaging that different acoustic pressures were needed to disrupt the capsules with varying shell thickness to diameter ratios. Also, the ultrasound behavior of these capsules was proven to be in excellent agreement with the recently developed theory of Marmottant et al. Additionally, we have also shown that capsules with different shell thicknesses to diameter ratios can be triggered independently from each other in a single system, giving the possibility to release drugs in a stepwise or selective manner. Since the US pressures applied were well below the maximum Mechanical Index allowed for diagnostic imaging, this implies that these capsules can be used to deliver drugs without safety hazards. 


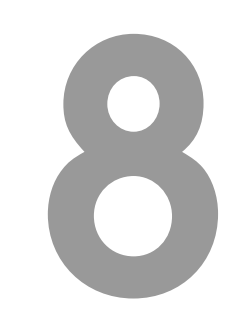

\section{Summary and outlook}

For several years, ultrasound contrast agents are being investigated for their therapeutic capacity. These gas-filled coated microbubbles have the ability to enhance local intravenous drug delivery. Microbubbles can be loaded with drugs by adding them to the bubble surface or by incorporating them into the core of the bubble. Upon microbubble oscillation and/or disruption, following ultrasound insonation, the drugs are released locally to achieve a therapeutic effect while avoiding systemic exposure. Furthermore, the interaction of oscillating microbubbles with the vascular endothelial layer causes an enhanced uptake of drugs by the surrounding tissue.

To visualize the process of ultrasound-triggered drug delivery, the use of ultrahigh-speed imaging is required to resolve the nanoseconds timescales involved. To obtain sufficient optical contrast and resolution to visualize the release of nanometer-sized particles, fluorescence microscopy has to be employed as well. Combining both techniques is challenging, due to the extremely short exposure time in which sufficient signal has to be collected from a limited amount of fluorescent material.

In this thesis the technique of ultra-high-speed fluorescence imaging is described. By combining laser-induced-fluorescence, employing a $5 \mathrm{~W}$ continuous wave (CW) laser, and the Brandaris 128 ultra-high-speed imaging facility a system was developed that allows for the visualization of fluorescently labeled microbubbles at a nanoseconds timescale. By focusing the laser light through the imaging microscope objective, excitation intensities on the order of megawatts per square centimeter were achieved. These excitation levels are sufficient to saturate all of the fluorescent material present on the fluorescently labeled microbubble, thereby achieving the maximum emission levels and allowing for fluorescence imaging at frame rates exceeding 10 million frames per second. Time-resolved imaging can be performed on a large range of timescales by using a gated $\mathrm{CW}$ laser. 
Fluorescence imaging can also aid in the study of microbubble dynamics. The bubble radius is an important parameter in theoretical models that describe the bubble behavior as a function of the applied ultrasound. However, in transmitted light microscopy, the typical imaging method for microbubble studies, the bubble image is distorted by scattering and diffraction of the illumination light by the bubble. This results in a complicated image from which it is difficult to determine the radius. When viewed in fluorescence the bubble image does not suffer from scattering or diffraction artifacts, since the fluorescence light is emitted omnidirectional and has little interaction with the bubble. The comparison of both techniques for optical sizing of microbubbles has lead to an empirical relation between the bubble radius obtained in bright field and fluorescence microscopy. This result was applied to correct the data from a recent bubble dynamics study.

To visualize the process of controlled drug release from a microbubble, ultrahigh-speed fluorescence recordings at $100000 \mathrm{fps}$ were performed. Liposomeloaded phospholipid-coated microbubbles were insonified by low-intensity ultrasound (20-100 kPa, 10-1000 cycles). The liposomes, drug-carrying vesicles with a typical diameter of $100 \mathrm{~nm}$, were loaded with a fluorescent dye to follow their position. The fluorescence recordings show that, below the threshold of release, the liposomes and phospholipids on the microbubble surface are highly mobile upon insonation and rearrange themselves over the bubble shell. Above the threshold two distinct types of release were observed both for unloaded and liposome-loaded microbubbles: the release of individually resolved micron-sized particles and the release of a mist of submicron particles. The two release mechanisms were mostly observed independent from each other and result in different release velocities and release distances of the shed fluorescent material.

The ultrasound-triggered, microbubble-induced uptake by a vascular endothelial cell takes place on a much longer timescale than the release from a bubble. Although ultrasound pulses with a peak-negative pressure of $200 \mathrm{kPa}$ and a duration of 10 cycles at a frequency of $1 \mathrm{MHz}$ are sufficient to induce permeabilization of the cell membrane, the subsequent inflow of propidium iodide (PI) occurs within several milliseconds. The PI then diffuses into the cell and it typically takes $15-20$ seconds for the cell to be fully stained. The induced pore is formed very locally and closes again after several seconds. The inflow rate of PI suggests that the size of the pore depends on the bubble radius, indicating that the amplitude of oscillation plays an important role in the process of sonoporation.

In addition to the visualization of the process of ultrasound-triggered local drug delivery, two designs of drug-carrying, hard-shelled ultrasound contrast 
agents were characterized. Both agents consist of polymeric microcapsules. The first design is dedicated to multimodal imaging by being suitable for both photoacoustic imaging and ultrasound imaging. By doping the capsule shell with a fluorescent dye, the capsule becomes susceptible both to pulsed and continuous laser excitation. Compared to conventional photoacoustic contrast agent nanoparticles, a single microcapsule can already produce an enhance acoustic signal, caused by vaporization. The response of a capsule to the laser irradiation was modeled and found to be in good agreement with the experimentally obtained results.

A second microcapsule design was developed for a two-step drug delivery system. High-speed recordings of microcapsules insonified by ultrasound revealed that the activation threshold of the capsules strongly correlates with the shell thickness to diameter ratio. By mixing capsules with two shell thickness to diameter ratios, selective triggering can be performed by applying different acoustic pressures. The measured thresholds correspond well to a recent theoretical model for hard-shelled agents by Marmottant et al. [127]

The application of ultra-high-speed fluorescence imaging for the visualization of microbubble phenomena has great potential. The matters addressed here represent only a small number of all possible experiments that can benefit from such an imaging technique. First, the study of bubble dynamics can be improved. For instance, the dynamics of fluorescent shell material during microbubble oscillations can be studied in more detail to acquire information on the properties of the phospholipid shell. Also, the vaporization process of perfluorcarbon nanodroplets could be resolved at a submicron resolution. Secondly, ultrasound-triggered drug release requires further research to fully resolve the physical mechanisms underlying the microbubble-induced therapeutic effect. Visualization of the release from a microcapsule with a payload in its core, such as the designs presented in chapter 6 and 7 , should be the next step in this process, alongside the study of surface loading configurations, such as liposomeloaded microbubbles. Finally, experiments aimed to assess the efficacy of drug uptake can be improved. Dynamic flow conditions instead of static flow conditions will enhance the applicability of in vitro experiments by mimicking the in vivo situation more realistically. Furthermore, the use of a fluorescent model drug that is transfected into a cell without affecting the cell viability is preferred over the use of PI. 


\section{Bibliography}

[1] B. B. Goldberg, J. B. Liu, and F. Forsberg. Ultrasound contrast agents: a review. Ultrasound Med. Biol., 20(4):319-333, 1994.

[2] N. de Jong, M. Emmer, C. T. Chin, A. Bouakaz, F. Mastik, D. Lohse, and M. Versluis. "Compression-Only" behavior of phospholipid-coated contrast bubbles. Ultrasound Med. Biol., 33(4):653-656, 2007.

[3] B. Dollet, S. M. van der Meer, V. Garbin, N. de Jong, D. Lohse, and M. Versluis. Nonspherical oscillations of ultrasound contrast agent microbubbles. Ultrasound Med. Biol., 34(9):1465-1473, 2008.

[4] V. Garbin, B. Dollet, M. Overvelde, D. Cojoc, E. Di Fabrizio, L. van Wijngaarden, A. Prosperetti, N. de Jong, D. Lohse, and M. Versluis. History force on coated microbubbles propelled by ultrasound. Phys. Fluids, 21(9):2003-2009, 2009.

[5] J. R. Lindner. Microbubbles in medical imaging: current applications and future directions. Nat. Rev. Drug Discov., 3:527-533, 2004.

[6] A. L.Klibanov. Microbubble Contrast Agents: Targeted ultrasound imaging and ultrasound-assisted drug-delivery applications. Invest. Radiol., 41(3):354-362, 2006.

[7] M. R. Böhmer, A. L. Klibanov, K. Tiemann, C. S. Hall, H. Gruell, and O. C. Steinbach. Ultrasound triggered image-guided drug delivery. Eur. J. Radiol., 70(2):242-253, 2009.

[8] I. Lentacker, S. C. De Smedt, and N. N. Sanders. Drug loaded microbubble design for ultrasound triggered delivery. Soft Matter, 5(11): 2161-2170, 2009.

[9] I. Lentacker, B. Geers, J. Demeester, S. C. De Smedt, and N. N. Sanders. Design and evaluation of doxorubicin-containing microbubbles for ultrasound-triggered doxorubicin delivery. Mol. Ther., 18(1):101-108, 2009.

[10] A. L.Klibanov, T. I. Shevchenko, B. I. Raju, R. Seip, and C. T. Chin. Ultrasound-triggered release of materials entrapped in microbubbleliposome constructs: A tool for targeted drug delivery. J. Contr. Rel., 148: 13-17, 2010. 
[11] M. S. Tartis, J. McCallan, A. F. Lum, R. LaBell, S. M. Stieger, T. O. Matsunaga, and K. W. Ferrara. Therapeutic effects of paclitaxel-containing ultrasound contrast agents. Ultrasound Med. Biol., 32:1771-1780, 2006.

[12] H. Leong-Poi, M. A. Kuliszewski, M. Lekas, M. Sibbald, K. TeichertKuliszewska, A. L. Klibanov, D. J. Stewart, and J. R. Lindner. Measurement of individual red blood cell motions under high hematocrit conditions using a confocal micro-PTV system. Circ. Res., 101:295-303, 2007.

[13] S. T. Wereley and C. D. Meinhart. Recent Advances in Micro-Particle Image Velocimetry. Ann. Rev. Fluid Mech., 42(1):557-576, 2010.

[14] K Shinohara, Y Sugii, A Aota, A Hibara, M Tokeshi, T Kitamori, and $\mathrm{K}$ Okamoto. High-speed micro-PIV measurements of transient flow in microfluidic devices. Meas. Sci. Technol., 15(10):1965-1970, 2004.

[15] C. T. Chin, C. Lancée, J. Borsboom, F. Mastik, M. E. Frijlink, N. de Jong, M. Versluis, and D. Lohse. Brandaris 128: A digital 25 million frames per second camera with 128 highly sensitive frames. Rev. Sci. Instr., 74(12):5026-5034, 2003.

[16] V. Parker and C. Roberts. In S. F. Ray, editor, High Speed Photography and Photonics. (SPIE, Bellingham, WA).

[17] E. A. Igel and M. Kristiansen. Rotating-mirror streak and framing cameras, volume PM43. (SPIE, Bellingham, WA), 1997.

[18] S. M. van der Meer, B. Dollet, M. M. Voormolen, C. T. Chin, A. Bouakaz, $\mathrm{N}$. de Jong, M. Versluis, and D. Lohse. Microbubble spectroscopy of ultrasound contrast agents. J. Acoust. Soc. Am., 121(1):648-56, 2007.

[19] P. Marmottant, S. M. van der Meer, M. Emmer, M. Versluis, N. de Jong, S. Hilgenfeldt, and D. Lohse. A model for large amplitude oscillations of coated bubbles accounting for buckling and rupture. J. Acoust. Soc. Am., 118(6):3499-3505, 2005.

[20] M. Emmer, A. van Wamel, D. E. Goertz, and N. de Jong. The onset of microbubble vibration. Ultrasound Med. Biol., 33(6):941-949, 2007.

[21] J. Sijl, B. Dollet, M. Overvelde, V. Garbin, T. Rozendal, N. de Jong, D. Lohse, and M. Versluis. Subharmonic behavior of phospholipid-coated ultrasound contrast agent microbubbles. The Journal of the Acoustical Society of America, 128(5):3239-52, 2010. 
[22] V. Garbin, D. Cojoc, E. Ferrari, E. Di Fabrizio, M. L. J. Overvelde, S. M. van der Meer, N. de Jong, D. Lohse, and M. Versluis. Changes in microbubble dynamics near a boundary revealed by combined optical micromanipulation and high-speed imaging. Appl. Phys. Lett., 90:114103, 2007.

[23] K. Kooiman, M. R. Böhmer, M. Emmer, H. J. Vos, C. Chlon, W. T. Shi, C. S. Hall, S. de Winter, K. Schroen, M. Versluis, N. de Jong, and A. van Wamel. Oil-filled polymer microcapsules for ultrasound-mediated delivery of lipophilic drugs. J. Contr. Rel., 133(2):109-118, 2009.

[24] A. van Wamel, K. Kooiman, M. Harteveld, M. Emmer, F. J. ten Cate, $\mathrm{M}$. Versluis, and $\mathrm{N}$. de Jong. Vibrating microbubbles poking individual cells: drug transfer into cells via sonoporation. J. Contr. Rel., 112(2): 149-155, 2006.

[25] W. van Hoeve, S. Gekle, J. H. Snoeijer, M. Versluis, M. P. Brenner, and D. Lohse. Breakup of diminutive Rayleigh jets. Phys. Fluids, 22(12): 122003, 2010.

[26] A. G. Zijlstra, T. Janssens, K. Wostyn, M. Versluis, P. W. Mertens, and D. Lohse. High speed imaging of $1 \mathrm{mhz}$ driven microbubbles in contact with a rigid wall. Solid State Phenom, 145(7):7-10, 2009.

[27] J. P. Crimaldi. Planar laser induced fluorescence in aqueous flows. Exp. Fluids, 44(6):851-863, 2008.

[28] A. Leipertz, A. Braeuer, J. Kiefer, A. Dreizler, and C. Heeger. Laserinduced fluorescence. In Handbook of combustion, pages 219-242. WileyVCH Verlag, 2010.

[29] F. C. De Lucia, R. S. Harmon, K. L. McNesby, R. J. Winkel, and A. W. Miziolek. Laser-induced breakdown spectroscopy analysis of energetic materials. Appl. Optics, 42(30):6148-6152, 2003.

[30] T. Ding, Th. H. van der Meer, M. Versluis, M. Golombok, J. Hult, M. Alden, and C. F. Kaminski. Time-resolved plif measurements in turbulent diffusion flames. In Y. Nagano, K. Hanjalic, and T. Tsuji, editors, Third International Symposium on Turbulence, Heat and Mass Transfer, pages 857-864.

[31] M. Overvelde, V. Garbin, J. Sijl, B. Dollet, N. de Jong, D. Lohse, and M. Versluis. Nonlinear shell behavior of phospholipid-coated microbubbles. Ultrasound Med. Biol., 36(12):2080-2092, 2010. 
[32] T. Faez, M. Emmer, M. Docter, J. Sijl, M. Versluis, and N. de Jong. Characterizing the subharmonic response of phospholipid-coated microbubble for carotid imaging. Ultrasound Med. Biol., 37(6):958-970, 2011.

[33] F. Guidi, H. J. Vos, R. Mori, N. de Jong, and P. Tortoli. Microbubble characterization through acoustically induced deflation. IEEE Trans. UItrason. Ferroelec. Freq. Contr., 57(1):193-202, 2010.

[34] A. L. Klibanov, P. T. Rasche, M. S. Hughes, J. K. Wojdyla, K. P. Galen, J. H. Wible, and G. H. Brandenburger. Detection of Individual Microbubbles of Ultrasound Contrast Agents. Invest. Radiol., 39(3):187-195, 2004.

[35] A. van der Bos, A. Zijlstra, E. Gelderblom, and M. Versluis. ilif: illumination by laser-induced fluorescence for single flash imaging on a nanoseconds timescale. Exp. Fluids, 51(5):1283-1289, 2011.

[36] Y. Tagawa, N. Oudalov, C. W. Visser, I. R. Peters, D. van der Meer, C. Sun, A. Prosperetti, and D. Lohse. Highly focused supersonic microjets. ArXiv e-prints, 2011.

[37] D. Maresca, M. Emmer, P. L. M. J. van Neer, H. J. Vos, M. Versluis, M. Muller, N. de Jong, and A. F. W. van der Steen. Acoustic sizing of an ultrasound contrast agent. Ultrasound Med. Biol., 36(10):1713-1721, 2010.

[38] R. W. DeBlois and C. P. Bean. Counting and sizing of submicron particles by resistive pulse technique. Rev. Sci. Instr., 41(7):909-916, 1970.

[39] J . A. Feshitan, C. C. Chen, J. J. Kwam, and M. A. Borden. Microbubble size isolation by differential centrifugation. J. Coll. Interf. Sci., 329:316324, 2009.

[40] J. Guan and T. J. Matula. Using light scattering to measure the response of individual ultrasound contrast microbubbles subjected to pulsed ultrasound in vitro. J. Acoust. Soc. Am., 116(5):2832-2842, 2004.

[41] M. R. Böhmer, R. Schroeders, J. A. M. Steenbakkers, S. H. P. M. de Winter, P. A. Duineveld, J. Lub, W. P. M. Nijssen, J. A. Pikkemaat, and H. R. Stapert. Preparation of monodisperse polymer particles and capsules by ink-jet printing. Colloid. Surface. A, 289(1-3):96-104, 2006.

[42] M. A. Borden, G. V. Martinez, J. Ricker, N. Tsvetkova, M. Longo, R. J. Gillies, P. A. Dayton, and K. W. Ferrara. Lateral phase separation in lipid-coated microbubbles. Langmuir, 22:4291-4297, 2006. 
[43] V. Sboros, E. Glynos, S. D. Pye, C. M. Moran, M. Butler, J. Ross, R. Short, W. N. McDicken, and V. Koutsos. Nanointerrogation of ultrasonic contrast agent microbubbles using atomic force microscopy. Ultrasound Med. Biol., 32(4):579-585, 2006.

[44] A. K. Jain. Fundamentals of digital image processing. Prentice-Hall, Englewood Cliffs, 1989.

[45] M. Postema, A. Bouakaz, C. T. Chin, and N. de Jong. Simulations and measurement of optical images of insonified ultrasound contrast agents. IEEE Trans. Ultrason. Ferroelec. Freq. Contr., 50(5):523-536, 2003.

[46] G. Mie. Contributions to the optics of turbid media, particularly of colloidal metal solutions. Ann. Phys., 25(3):377-455, 1908.

[47] S. Inoue and R. Oldenbourg. Handbook of Optics II: Devices, measurements and properties, chapter Microscope. McGraw-Hill, New York, 1995.

[48] Y. Sun, S. Duthaler, and B. J. Nelson. Autofocus in computer microscopy: selecting the optimal focus algorithm. Microsc. Res. Tech., 65:139-149, 2004.

[49] M. W. Davidson and M. Abramowitz. Encyclopedia of Imaging Science and Technology, chapter Optical Microscopy, pages 1106-1141. WileyInterscience, New York, 2002.

[50] J. Sijl, H. J. Vos, T. Rozendal, N. de Jong, D. Lohse, and M. Versluis. Combined optical and acoustical detection of single microbubble dynamics. J. Acoust. Soc. Am., 130(5):3271-3281, 2011.

[51] C. A. Sennoga, V. Mahue, J. Loughran, J. Casey, J. M. Seddon, M. Tang, and R. J. Eckersley. On sizing and counting of microbubbles using optical microscopy. Ultrasound Med. Biol., 36(12):2093-2096, 2010.

[52] H. J. Vos, B. Dollet, J. G. Bosch, M. Versluis, and N. de Jong. Nonspherical vibrations of microbubbles in contact with a wall - a pilot study at low mechanical index. Ultrasound Med. Biol., 34(4):685-688, 2008.

[53] H. J. Vos, B. Dollet, M. Versluis, and N. de Jong. Nonspherical shape oscillations of coated microbubbles in contact with a wall. Ultrasound Med. Biol., 37(6):935-948, 2011.

[54] S. Kaul. Myocardial contrast echocardiography: a 25-year retrospective. Circulation, 118:291-308, 2008. 
[55] J. A. Jakobsen, R. Oyen, H. S. Thomsen, and S. K. Morcos. Safety of ultrasound contrast agents. Eur Radiol, 15:941-945, 2005.

[56] M. Dyson, J. B. Pond, J. Joseph, and R. Warwick. Stimulation of tissue regeneration by means of ultrasound. Clin. Sci., 35:273-285, 1968.

[57] S. Tachibana and S. Tachibana. Albumin microbubble echo-contrast material as an enhancer for ultrasound accelerated thrombolysis. Circulation, 92:1148-1150, 1995.

[58] S. Hernot and A. L. Klibanov. Microbubbles in ultrasound-triggered drug and gene delivery. Adv. Drug Del. Rev., 60(10):1153-1166, 2008.

[59] S. Tinkov, R. Bekeredjian, G. Winter, and C. Coester. Microbubbles as ultrasound triggered drug carriers. J. Pharm. Sci, 98(6):1935-1961, 2009.

[60] A. Lawrie, A. F. Brisken, S. E. Francis, D. C. Cumberland, D. C. Crossman, and C. M. Newman. Microbubble-enhanced ultrasound for vascular gene delivery. Gene Ther., 7:2023-2027, 2000.

[61] M. Schneider. Molecular imaging and ultrasound-assisted drug delivery. J. Endourol., 22(4):795-801, 2008.

[62] K. Hynynen. Ultrasound for drug and gene delivery to the brain. Adv. Drug Del. Rev., 60(2):1209-1217, 2005.

[63] D. Lensen, E. Gelderblom, D. Vriezema, P. Marmottant, N. Verdonschot, M. Versluis, N. de Jong, and J. van Hest. Biodegradable polymeric microcapsules for selective ultrasound-triggered drug release. Soft Matter, 7:5417-5422, 2011.

[64] P. Hauff, S. Seemann, R. Reszka, M. Schultze-Mosqau, M. Reinhardt, T. Buzasi, T. Plath, S. Rosewicz, and M. Schirner. Evaluation of gasfilled microparticles and sonoporation as gene delivery system: feasibility study in rodent tumor models. Radiology, 236(2):572-578, 2005.

[65] E. C. Unger, T. P. McCreery, R. H. Sweitzer, V. E. Caldwell, and Y. Wu. Acoustically active lipospheres containing paclitaxel: a new therapeutic ultrasound contrast agent. Invest. Radiol., 33(12):886-892, 1998.

[66] E. C. Unger, T. P. McCreery, R. H. Sweitzer, G. Wu, D. Shen, and D. Yellowhair. Mrx 501: a novel ultrasound contrast agent with therapeutic properties. Acad. Radiol., 5:S247-S249, 1998. 
[67] A. Kheirolomoom, P. A. Dayton, A. F. H. Lum, E. Little, E. E. Paoli, $\mathrm{H}$. Zheng, and K. W. Ferrara. Microbubble-enhanced ultrasound for vascular gene delivery. J. Contr. Rel., 118:275-284, 2007.

[68] B. Geers, I. Lentacker, N.N. Sanders, J. Demeester, S. Meairs, and S.C. De Smedt. Self-assembled liposome-loaded microbubbles: The missing link for safe and efficint ultrasound triggered drug-delivery. J. Contr. Rel., 152:249-256, 2011.

[69] R. E. Vandenbroucke, I. Lentacker, J. Demeester, S. C. De Smedt, and N. N. Sanders. Ultrasound assisted sirna delivery using peg-siplex loaded microbubbles. J. Contr. Rel., 126:265-273, 2008.

[70] M. L. De Temmerman, H. Dewitte, R. E. Vandenbroucke, B. Lucas, C. Libert, J. Demeester, S. C. De Smedt, I. Lentacker, and J. Rejman. mrna-lipoplex loaded microbubble contrast agents for ultrasound-assisted transfection of dendritic cells. Biomaterials, 32:9128-9135, 2011.

[71] M. A. Borden, G. Pu, G. J. Runner, and M. L. Longo. Surface phase behavior and microstructure of lipid/peg-emulsifier monolayer-coated microbubbles. Colloid. Surface. B, 35:209-223, 2004.

[72] G. Pu, M. A. Borden, and M. L. Longo. Collapse and shedding transitions in binary lipid monolayers coating microbubbles. Langmuir, 22:29932999, 2006.

[73] Y. Luan, T. Faez, E. C. Gelderblom, I. Skachkov, B. Geers, I. Lentacker, T. van der Steen, M. Versluis, and N. de Jong. Investigating the acoustical properties of individual liposome-loaded microbubbles by ultra-high speed imaging. Ultrasound Med. Biol. (submitted), 2012.

[74] C. A. Naumann, W. Knoll, and C. W. Frank. Hindered diffusion in polymer-tethered membranes: A monolayer study at the air-water interface. Biomacromolecules, 2:1097-1103, 2001.

[75] P. Marmottant, M. Versluis, N. de Jong, S. Hilgenfeldt, and D. Lohse. High-speed imaging of an ultrasound-driven bubble in contact with a wall: narcissus" effect and resolved acoustic streaming. Exp. Fluids, 41: 147-153, 2006.

[76] P. Marmottant, J. P. Raven, H. Gardeniers, J. G. Bomer, and S. Hilgenfeldt. Microfluidics with ultrasound-driven bubbles. J. Fluid Mech., 568: 109-118, 2006. 
[77] P. Marmottant and S. Hilgenfeldt. Controlled vesicle deformation and lysis by single oscillating bubbles. Nature, 423:153-156, 2003.

[78] S. W. Fong, E. Klaseboer, C. K. Turangan, B. C. Khoo, and K. C. Hung. Analysis of a gas bubble near bio-materials in an ultrasound field. Ultrasound Med. Biol., 32:925-942, 2006.

[79] M. Versluis, D. E. Goertz, P. Palanchon, I. L. Heitman, S. M. van der Meer, B. Dollet, N. de Jong, and D. Lohse. Microbubble shape oscillations excited through ultrasonic parametric driving. Phys. Rev. E, 82:026321$1-6,2010$.

[80] S. Bao, B. D. Thrall, and D. L. Miller. Transfection of a reporter plasmid into cultured cells by sonoporation in vitro. Ultrasound Med. Biol., 23 (6):953-959, 1997.

[81] M. Ward, J. Wu, and J. F. Chiu. Ultrasound-induced cell lysis and sonoporation enhanced by contrast agents. J. Acoust. Soc. Am., 105 (5):2951-2957, 2009.

[82] Y. Zhou, R. E. Kumon, J. Cui, and C. X. Deng. The size of sonoporation pores on the cell membrane. Ultrasound Med. Biol., 35(10):1756-1760, 2009.

[83] P. Prentice, A.Cuschieri, K.Dholakia, M. Prausnitz, and P. Campbell. Membrane disruption by optically controlled microbubble cavitation. Nat. Phys., 1:107-110, 2005.

[84] K. Kooiman, M. Foppen-Harteveld, A. F. W. van der Steen, and N. de Jong. Sonoporation of endothelial cells by vibrating targeted microbubbles. J. Contr. Rel., 154:35-41, 2011.

[85] J. P. Ross, X. Cai, J. F. Chiu, J. Yang, and J. Wu. Optical and atomic force microscopic studies on sonoporation. J. Acoust. Soc. Am., 111(3): 1161-1165, 2002.

[86] D. L. Miller and C. Dou. Induction of apoptosis in sonoporation and ultrasonic gene transfer. Ultrasound Med. Biol., 35(1):144-154, 1999.

[87] H. Pan, Y. Zhou, O. Izadnegahdar, J. Cui, and C. X. Deng. Study of sonoporation dynamics affected by ultrasound duty cycle. Ultrasound Med. Biol., 31(6):849-856, 2005. 
[88] T. Suzuki, K. Fujikura, T. Higashiyama, and K. Takata. Dna staining for fluorescence and laser confocal microscopy. J. Histochem. Cytochem., 45 (1):49-53, 1997.

[89] Y. Z. Zhao, J. Oczos, P. Janowski, D. Trembecka, J. Dobrucki, Z. Darzynkiewicz, and D. Wlodkowic. Rationale for the real-time and dynamic cell death assays using propidium iodide. 77:399-405, 2010.

[90] P. D. Bevan R. Karshafian, R. Williams, S. Samac, and P. N. Burns. Sonoporation by ultrasound-activated microbubble contrast agents: effect of acoustic exposure parameters on cell membrane permeability and cell viability. Ultrasound Med. Biol., 35(5):847-860, 2009.

[91] M. M. Forbes, R. L. Steinberg, and W. D. O'Brien. Examination of inertial cavitation of optison in producing sonoporation of chinese hamster ovary cells. Ultrasound Med. Biol., 34(12):2009-2018, 2008.

[92] S. Mehier-Humbert, T. Bettinger, F. Yan, and R. H. Guy. Plasma membrane poration induced by ultrasound exposure: implication for drug delivery. J. Contr. Rel., 104:213-222, 2005.

[93] N. Kudo, K. Okada, and K. Yamamoto. Sonoporation by single-shot pulsed ultrasound with microbubbles adjacent to cells. Biophys. J., 96: 4866-4876, 2009.

[94] A. Yudina, M. Lepetit-Coiffe, and C. T. W. Moonen. Evaluation of the temporal window for drug delivery following ultrasound-mediated membrane permeability enhancement. 13:239-249, 2011.

[95] T. A. Tran, S. Roger, J. Y. Le Guennec, F. Tranquart, and A. Bouakaz.

[96] K. Ogawa, K. Tachibana, T. Uchida, T. Tai, N. Yamashita, N. Tsujita, and R. Miyauchi. High-resolution scanning electron microscopic evaluation of cell-membrane porosity by ultrasound. Med. Electron. Microsc., 34:249-253, 2001.

[97] R. K. Schlicher, H. Radhakrishna, T. P. Tolentino, R. P. Apkarian, V. Zarnitsyn, and M. R. Prausnitz. Mechanism of intracellular delivery by acoustic cavitation. Ultrasound Med. Biol., 32(6):915-924, 2006.

[98] Y. Z. Zhao, Y. K. Luo, C. T. Lu, J. F. Xu, J. Tang, M. Zhang, Y. Zhang, and H. D. Liang. Phospholipids-based microbubbles sonoporation pore size and reseal of cell membrane cultured in vitro. 16:18-25, 2008. 
[99] E. A. Jaffe, R. L. Nachman, C. G. Becker, and C. R. Minick. Culture of human endothelial cells derived from umbilical veins. identification by morphologic and immunologic criteria. J. Coll. Interf. Sci., 52(11):27452756, 1973.

[100] M. Minnaert. On musical air-bubbles and sounds of running water. Philos. Mag., 16:235-248, 1933.

[101] M. Strasberg. The pulsation frequency of nonspherical gas bubble in liquids. J. Acoust. Soc. Am., 25(3):536-537, 1953.

[102] W. Lauterborn. Numerical investigation of nonlinear oscillations of gas bubbles in liquids. J. Acoust. Soc. Am., 59(2):283-293, 1976.

[103] X. Wang, Y. Pang, G. Ku, X. Xie, G. Stoica, and L. V. Wang. Noninvasive laser-induced photoacoustic tomography for structural and functional in vivo imaging of the brain. Nat. Biotechnol., 21:803-806, 2003.

[104] X. Wang, X. Xie, G. Ku, and L. V. Wang. Noninvasive imaging of hemoglobin concentration and oxygenation in the rat brain using highresolution photoacoustic tomography. J. Biomed. Opt., 11(2):024015, 2006.

[105] K. Jansen, A. F. W. van der Steen, H. M. M. van Beusekom, J. W. Oosterhuis, and G. van Soest. Intravascular photoacoustic imaging of human coronary atherosclerosis. Opt. Lett., 36(5):597-599, 2011.

[106] S. Manohar, S. E. Vaartjes, J. C. G. van Hespen, J. m. Klaase, F. M. van den Engh, W. Steenbergen, and T. G. van Leeuwen. Initial results of in vivo non-invasive cancer imaging in the human breast using near-infrared photoacoustics. Opt. Express, 15(19):12277-12285, 2007.

[107] M. Eghtedari, A. Oraevsky, J. A. Copland, N. A. Kotov, A. Conjusteau, and M. Motamedi. High sensitivity of in vivo detection of gold nanorods using a laser optoacoustic imaging system. Nano Lett., 7(7):1914-1918, 2007.

[108] K. Kim, S. W. Huang, S. Ashkenazi, M. O'Donnell, and A. Agarwal. Photoacoustic imaging of early inflammatory response using gold nanorods. Appl. Phys. Lett., 90:223901, 2007.

[109] J. C. Chappell, J. Song, C. W. Burke, A. L. Klibanov, and R. J. Price. Targeted delivery of nanoparticles bearing fibroblast growth factor-2 by ultrasonic microbubble destruction for therapeutic arteriogenesis. Small, 4(10):1769-1777, 2008. 
[110] C. Chlon, C. Gudon, B. Verhaagen, W. T. Shi, C. S. Hall, J. Lub, and M. R. Böhmer. Effect of molecular weight, crystallinity, and hydrophobicity on the acoustic activation of polymer-shelled ultrasound contrast agents. Biomacromolecules, 10(5):1025-31, 2009.

[111] T. G. Leighton. The acoustic bubble. Academic Press, London, 1994.

[112] A. Kawski, B. Kuklinski, and P. Bojarski. Photophysical properties and thermochromic shifts of electronic spectra of nile red in selected solvents. excited states dipole moments. Chem. Phys., 359(1-3):58-64, 2009.

[113] A. Y. Jee, S. Park, H. Kwon, and M. Lee. Excited state dynamics of nile red in polymers. Chem. Phys. Lett., 477(1-3):112-115, 2009.

[114] P. Stchur, K. X. Yang, X. Hou, T. Sun, and R. G. Michel. Laser excited atomic fluorescence spectrometry. Spectrochim. Acta, Part B, 56(9): 1565-1592, 2001.

[115] V. Sboros. Response of contrast agents to ultrasound. Adv. Drug Del. Rev., 60(10):1117-1136, 2008.

[116] E. G. Schutt, D. H. Klein, R. M. Mattrey, and J. G. Riess. Injectable microbubbles as contrast agents for diagnostic ultrasound imaging: the key role of perfluorochemicals. Angew. Chem., Int. Ed., 42(28):32183235, 2003.

[117] J. E. Chomas, P. A. Dayton, D. May, J. Allen, A. Klibanov, and K. Ferrara. Optical observation of contrast agent destruction. Appl. Phys. Lett., 77(7):1056-1058, 2000.

[118] C. C. Church. The effects of an elastic solid surface layer on the radial pulsations of gas bubbles. J. Acoust. Soc. Am., 97(3):1510-1521, 1995.

[119] A. A. Doinikov and P. A. Dayton. Maxwell rheological model for lipidshelled ultrasound microbubble contrast agents. J. Acoust. Soc. Am., 121(6):3331-3340, 2007.

[120] A. A. Doinikov, J. F. Haac, and P. A. Dayton. Modeling of nonlinear viscous stress in encapsulating shells of lipid-coated contrast agent microbubbles. Ultrasonics, 49(2):269-275, 2009.

[121] K. Sarkar, W. T. Shi, D. Chatterjee, and F. Forsberg. Characterization of ultrasound contrast microbubbles using in vitro experiments and viscous and viscoelastic interface models for encapsulation. J. Acoust. Soc. Am., 118(1):539-550, 2005. 


\section{BIBLIOGRAPHY}

[122] E. Stride and N. Saffari. On the destruction of microbubble ultrasound contrast agents. Ultrasound Med. Biol., 29(4):563-573, 2003.

[123] A. Bouakaz, M. Versluis, and N. de Jong. High-speed optical observations of contrast agent destruction. Ultrasound Med. Biol., 31(3):391-399, 2005.

[124] C. K. Yeh and S. Y. Su. Effects of acoustic insonation parameters on ultrasound contrast agent destruction. Ultrasound Med. Biol., 34(8): 1281-1291, 2008.

[125] S. H. Bloch, M. Wan, P. A. Dayton, and K. W. Ferrara. Optical observation of lipid- and polymer-shelled ultrasound microbubble contrast agents. Appl. Phys. Lett., 84:631-633, 2004.

[126] W. K. Lee, I. Losito, J. A. Gardella, and W. L. Hicks. Synthesis and surface properties of fluorocarbon end-capped biodegradable polyesters. Macromolecules, 34(9):3000-3006, 2001.

[127] P. Marmottant, A. Bouakaz, N. de Jong, and C. Quilliet. Buckling resistance of solid shell bubbles under ultrasound. J. Acoust. Soc. Am., 129 (3):1231-1239, 2011.

[128] M. R. Böhmer, C. H. T. Chlon, B. I. Raju, C. T. Chin, T. Shevchenko, and A. L. Klibanov. Focused ultrasound and microbubbles for enhanced extravasation. J. Contr. Rel., 148(1):18-24, 2010.

[129] R. E. Apfel and C. K. Holland. Gauging the likelihood of cavitation from short-pulse, low-duty cycle diagnostic ultrasound. Ultrasound Med. Biol., 17(2):179-185, 1991.

[130] FDA. Information for manufacturers seeking marketing clearance of diagnostic ultrasound systems and transducers. http://www.fda.gov/downloads/UCM070911.pdf., September 2008.

[131] D. Lensen, D. M. Vriezema, and J. C. M. van Hest. Polymeric microcapsules for synthetic applications. Macromol. Biosci., 8(11):991-1005, 2008.

[132] D. Lensen, K. van Breukelen, D. M. Vriezema, and J. C. M. van Hest. Preparation of biodegradable liquid core plla microcapsules and hollow plla microcapsules using microfluidics. Macromol. Biosci., 10(5):475480, 2010. 


\section{Samenvatting}

Om medische diagnostiek met behulp van ultrageluid te verbeteren, worden sinds de jaren tachtig belletjes gebruikt, die het contrast van bloed op ultrageluidsafbeeldingen sterk verhogen. Deze microscopisch kleine gasbellen worden intravasculair toegediend en daarmee kan de doorbloeding van organen beter zichtbaar worden gemaakt. De microbellen reflecteren het ultrageluid vele malen beter dan weefsel door de hoge compressibiliteit van het gas in de bel. Daarnaast resoneren microbellen rond de frequentie van het ingestraalde ultrageluid, waardoor het gereflecteerde signaal nog verder wordt versterkt. In hart echoscopie kan hierdoor bijvoorbeeld een slecht doorbloed gedeelte van de hartspier eenvoudig worden gelokaliseerd.

Sinds een aantal jaar worden microbellen onderzocht voor therapeutische toepassingen. Door medicijnen in de bel of aan de schil van de bel te bevestigen, kunnen medicijnen getransporteerd worden. Met ultrageluid kunnen de bellen vervolgens op de precieze locatie in het lichaam kapot worden gemaakt, zodat het medicijn heel lokaal wordt afgegeven. Bovendien zorgen de bellen voor een verhoogde opname door de omliggende vasculaire endotheelcellen, wanneer ze dichtbij de vaatwand trillen.

Het is uit eerder onderzoek gebleken dat het mogelijk is om heel gericht medicijnen toe te dienen met behulp van microbellen en ultrageluid. Echter, een goede beschrijving van de onderliggende fysische mechanismen ontbreekt op dit moment. Om meer kennis te vergaren over lokale medicijn toediening is het noodzakelijk om het proces in beeld te brengen. Vanwege de kleine afmetingen van de bellen is hiervoor microscopie vereist. De tijdschaal van de beloscillaties met een trilfrequentie van een megahertz is in de orde van nanoseconden, waardoor ook het gebruik van een ultra-hogesnelheidscamera noodzakelijk is.

Voor het bestuderen van de dynamica van oscillerende bellen worden hogesnelheidsopnamen veelvuldig gebruikt in combinatie met (helderveld transmissie) lichtmicroscopie. Echter, deze techniek zorgt voor onvoldoende optisch contrast om medicijnen en andere deeltjes, met een grootte in de orde van nanometers, te kunnen onderscheiden. Met behulp van fluorescentiemicroscopie kunnen medicijnen, gekoppeld aan fluorescente deeltjes, wel zichtbaar worden gemaakt. De lichtopbrengst in fluorescentiemicroscopie is doorgaans enkele ordes lager dan in lichtmicroscopie en wordt grotendeels bepaald door de concentratie van fluorescente deeltjes en de licht intensiteit. De beperkte hoeveelheid beschikbare fluorescente kleurstof in of op een bel en de zeer korte belichtingstijden, in de orde van nanoseconden, maken het daarom erg uitdagend om op hoge snelheid fluorescentiemicroscopie te doen. 


\section{SAMENVATTING}

In dit proefschrift wordt een experimentele methode gepresenteerd die het mogelijk maakt om fluorescente microbellen te visualiseren op een snelheid van meer dan 10 miljoen beelden per seconde. Om dit bereiken is een continue laser, met een vermogen van $5 \mathrm{~W}$, gekoppeld aan de Brandaris 128 ultrahogesnelheidscamera. Het laserlicht wordt gefocusseerd door het microscoop objectief, waarmee een extreem hoge licht intensiteit gegenereerd kan worden. Deze intensiteit is voldoende om alle aanwezige fluorescentie moleculen op de bel in verzadiging te brengen, zodat de maximale fluorescentie emissie wordt bereikt. Normaliter worden gepulste lasers ingezet om op een nanoseconde tijd-schaal fluorescentiemicroscopie te doen, echter, deze lasers zijn ongeschikt vanwege hun puls herhalingsfrequentie van hooguit $100 \mathrm{kHz}$. Door gebruik te maken van een continue laser is het systeem niet gelimiteerd in snelheid. Bovendien zijn deze lasers compacter en goedkoper dan gepulste lasers.

Fluorescentiemicroscopie kan ook gebruikt worden om het trilgedrag van de microbellen beter in beeld te krijgen. De belstraal is een belangrijke parameter voor theoretische modellen die het gedrag van een bel beschrijven aan de hand van het aangelegde ultrageluidsveld. Echter, het nauwkeurig meten van de belstraal in lichtmicroscopie is ingewikkeld, omdat de uiteindelijke afbeelding van de bel wordt bepaald door verstrooiing en diffractie van de lichtstralen afkomstig van de lichtbron. In fluorescentiemicroscopie is de afbeelding van de bel eenvoudiger, omdat het emissie licht in alle richtingen wordt uitgezonden en er vrijwel geen interactie is met de bel. Door beide technieken met elkaar te vergelijken is een empirisch verband gevonden voor de belstraal in fluorescentieen in lichtmicroscopie. Dit verband is toegepast om de experimentele resultaten van een recent onderzoek naar beldynamica, opgenomen in lichtmicroscopie, te corrigeren.

Het onderzoek naar medicijnafgifte met behulp van microbellen en ultrageluid kan worden opgedeeld in twee delen, die hier allebei worden behandeld. Het eerste deel betreft het vrijkomen van een (model) medicijn van de bel. Hiervoor zijn microbellen, geladen met liposomen, blootgesteld aan laag vermogen ultrageluid (20-100 kPa, 10-1000 periodes). De liposomen, medicijndragende deeltjes met een diameter in de orde van $100 \mathrm{~nm}$, waren voorzien van een fluorescente kleurstof om ze te kunnen volgen gedurende de beloscillaties. Hogesnelheid fluorescentie opnamen op 100000 beelden per seconde hebben laten zien dat het vrijkomen van liposomen op twee verschillende manieren gebeurt. Enerzijds komen losse deeltjes vrij met een grootte tot een micrometer. Anderzijds wordt een wolk van moleculaire deeltjes door de bel afgescheiden. Bovendien lijken de twee methodes alleen onafhankelijk van elkaar voor te komen.

Het tweede onderzoeksdeel van de medicijnafgifte betreft de opname van 
een (model) medicijn door de omliggende cel. Deze vindt plaats op een veel langere tijdschaal dan het vrijkomen van het medicijn. Opname van het medicijn door de cel, ten gevolge van een lokaal verhoogde permeabiliteit van het celmembraan, kan al worden veroorzaakt door beloscillaties aangedreven door ultrageluid met een druk van $200 \mathrm{kPa}$ en een pulsduur van 10 periodes bij een frequentie van $1 \mathrm{MHz}$. Niettemin wijzen fluorescente opnamen van de aankleuring van propidium jodide $(\mathrm{PI})$ in de cel erop dat de instroom pas op een tijdschaal van milliseconden begint. Vervolgens diffundeert de $\mathrm{PI}$ verder de cel in tot de gehele cel is aangekleurd, binnen 15-20 seconden. We hebben ontdekt dat de porie in het celmembraan waardoor de $\mathrm{PI}$ naar binnen stroomt, zich beperkt tot het contactpunt tussen de bel en de cel. Bovendien sluit de porie op een tijdschaal van seconden na toediening van het ultrageluid. De gemeten instroomsnelheid van PI suggereert dat de grootte van de porie afhankelijk is van de belstraal en de amplitude van beloscillaties.

Tot slot worden in hoofdstuk 6 en 7 twee typen microbellen gepresenteerd en gekarakteriseerd. Beide typen bellen kunnen medicijnen vervoeren in hun kern en hebben een harde schil die is opgebouwd uit polymeren. De eerste bel kan worden ingezet als contrastmiddel voor zowel diagnostisch ultrageluid als voor fotoakoestiek. Een fluorescente kleurstof, die in de schil van de bel is verwerkt, zorgt ervoor dat de bel op gepulste en ook op continue laser excitatie reageert. De dynamica van de gegenereerde dampbel is gemodelleerd en de resultaten hiervan komen goed overeen met de experimenten. Het tweede type bel is ontworpen als een tweetraps raket. Hogesnelheidsopnamen hebben aangetoond dat de drempelwaarde voor het openbreken van de polymere bel sterk afhangt van de verhouding van de schildikte en de beldiameter. In een mengsel van bellen met verschillende schildiktes kan dus selectief een gedeelte van de bellen worden opengebroken door ultrageluid met verschillende akoestische drukken toe te passen. De gemeten drempelwaardes komen overeen met een onlangs ontwikkeld model voor microbellen met een harde schil. 
SAMENVATTING 


\section{Dankwoord}

Ik ben niet iemand die lang in het verleden blijft hangen. Ik kijk graag vooruit naar wat er komen gaat: nieuwe ontdekkingen en belevenissen. Daarom is het best gek dat ik er voor gekozen heb om vier jaar lang experimentele natuurkunde te bedrijven, in het bijzonder met hogesnelheidscamera's. Al die plaatjes en filmpjes van fluorescente belletjes zijn ten slotte een weergave van gebeurtenissen die al voltooid zijn. Ze voltrokken zich weliswaar binnen een tijdsbestek dat nog vele malen korter is dan een enkele keer knipperen met je ogen, maar wat gebeurd is, kan niet meer ongedaan gemaakt. Daarom lag de uitdaging (lees: lol) met name in de voorbereiding: dagen-, weken- of soms maandenlang bedenken, tekenen, rekenen en testen om maar aan alles gedacht te hebben dat fout kón en goed móest gaan. Als dan het experiment was geslaagd, vormden de opnames een welkom moment van vreugde en bewondering, maar ondertussen was de voorbereiding voor het volgende experiment stiekem alweer gestart.

Dit proces van continu vooruitdenken en vernieuwen heb ik mogen doen bij de Physics of Fluids groep van Detlef Lohse. Ik ben hem zeer dankbaar voor de geboden kansen en het vertrouwen in mijn werk. De faciliteiten die hij heeft gecreerd zijn bewonderenswaardig. Tel daar een team van excellente wetenschappers bij op en je krijgt een unieke plek om wetenschap in al haar facetten te beleven.

Echter, met wetenschap alleen red je het niet in deze wereld. Daarom mag PoF zich gelukkig prijzen met Gert-Wim, Martin, Bas en Joanita die probleemloos de vertaalslag kunnen maken van idee naar praktijk, het - soms letterlijke - vuile werk opknappen en achter de schermen alles in goede banen leiden, dag in dag uit.

Dit proefschrift is slechts een kleine bijdrage aan het wereldwijde onderzoek naar belletjes voor medisch ultrageluid. Tegelijkertijd weet ik dat de studies die voortkomen uit de samenwerking tussen Twente en Rotterdam een grote impact hebben binnen het internationale onderzoeksveld. Ik heb dan ook veel geleerd van Marlies, Jeroen, Timo, Valeria, Benjamin, Alex en Guillaume, maar bovenal veel plezier met ze gehad. Datzelfde geldt voor Rik, Telli, Ying, Tom, Klazina, Geert, Robert en Frits, de ideale sparring partners voor die leu oet Twente. Daarnaast hebben Floor, Srirang, Ine, Heleen en Dennis er aan bijgedragen dat ik verder dan de muren van lab 207B heb gekeken om zo het proefschrift meer kleur te geven en mijn werkveld te verbreden.

Dat wil niet zeggen dat je altijd lang of ver hoeft te zoeken om de juiste personen te vinden. Samen met mijn (ex-)kantoorgenoten Arjan, Aaldert, Tim en Guillaume, van het door Detlef tot 'the cheerful office' uitgeroepen Mean- 


\section{DANKWOORD}

der 214, hoop ik in de loop van dit jaar maar liefst drie publicaties te hebben volbracht. Dit toont nog maar eens aan dat ongebreideld lol met elkaar hebben alleen maar ten goede komt aan de productiviteit. Wat dat betreft heb ik het ook getroffen met mijn 'dagelijks' begeleiders. Nico, je bent altijd enthousiast bij het zien van nieuwe resultaten, ongeacht of het een wetenschappelijk doel dient (dit is dan wel je eerste vraag) en je weet, juist door voldoende afstand te bewaren, goed een kritische noot te plaatsen bij de uitkomsten. Michel, je bent onmisbaar geweest in de ondersteuning en sturing van het wetenschappelijke proces en gaf mij tegelijkertijd de ruimte om te doen wat ik dacht dat nodig was om het doel te bereiken. Naast de inhoudelijke discussies heb ik vooral veel opgestoken van je vaardigheden op het gebied van de presentatie van wetenschap in de breedste zin van het woord. Ik heb enorm genoten van alle reizen en ik hoop jouw ontspannen houding mijzelf ook eigen te maken.

Ik ben altijd met plezier naar m'n werk gegaan, maar heb ook nooit met tegenzin de labdeur achter me dichtgedaan. Aan de andere kant daarvan stond namelijk altijd een ongelooflijk leuke groep van collega's klaar, goed voor vermaak tijdens borrels, vrijdagmiddag experimenten, fietstochten, het opnemen van promotievideo's en het bezoeken van conferenties. Wat een geweldige tijd!

Een promotie is tijdelijk, maar wanneer je collega's je vrienden worden, kun je tot in lengte van dagen blijven discussiren over het nut ervan. Onder het genot van de juiste koolzuurbelletjes en met vrienden als Marlies, Arjan, Aaldert, Rory en Wim is dat totaal geen straf. Minstens zoveel waarde hecht ik aan alle gezellige avonden met de stuntpiloten Elbert, Koen en Christian. Het lijkt misschien een kinderachtig spelletje, maar er gaat zoveel meer schuil achter. En Luc, ik wil daarom ook hier mijn waardering uitspreken voor al onze filmavonden en tennispartijen: thanks bro.

Ik wil ook heel graag mijn ouders bedanken voor alle ondersteuning, niet alleen in de afgelopen vier jaar, maar ook in alle jaren daarvoor. Jullie hebben me geleerd om te doen wat ik leuk vind maar bovenal mij de kans gegeven om dat te realiseren.

De laatste regels van dit proefschrift zijn gereserveerd voor de meest bijzondere vrouw in mijn leven. Lonneke, je geeft me iedere dag het gevoel dat ik de hele wereld aankan. Je haalt het beste in mij naar boven en probeert me telkens ietsje wijzer te maken. Wanneer ik een glimlach op jouw gezicht zie, weet ik dat ik op de goede weg zit.

- Erik - 